\title{
High-field EPR and ENDOR spectroscopy for proton-coupled electron transfer investigations in E.coli ribonucleotide reductase
}

\author{
Dissertation \\ for the award of the degree \\ “Doctor rerum naturalium” (Dr.rer.nat.) \\ Division of Mathematics and Natural Sciences \\ of the
}

Georg-August-Universität Göttingen

submitted by

Tomislav Argirević

from Bad Nauheim

Göttingen, 2011 
Referentin/Referent: Prof.Bennati

Koreferentin/Koreferent: Prof.Meyer

Tag der mündlichen Prüfung: 
За моје родитеље 


\section{Acknowledgment}

First of all I would like to thank my supervisor Marina Bennati. I will always appreciate what she invested in me when I started to become part of one of the most interesting biochemical projects, the ribonucleotide reductase project. In EPR spectroscopy her view for details and her experience was vital for me and it was always impressing to see, to understand and to learn from her how far you need to go in order to come to the final conclusion. There are no parallels for her persistence in facing a scientific problem. I thank her in motivating me to go to MIT, in putting a lot of trust in me in order to face a challenging PhD project and in supporting me to push the high-field EPR/ENDOR investigations on E.coli RNR as far as possible.

The research stay at MIT would not be feasible without JoAnne Stubbe. In her lab I was able to perform in a short time exciting science. Thank you a lot for this and for keeping our collaboration alive. Many, many thanks to Mohammad Seyedsayamdost, Clement Chan, Ellen Minnihan and Kenichi Yokoyama who gave many advices and who had always the right answer to my questions about RNR. Thanks also to Sumit Chakraborty, Rachael Buckley, Joey Cotruvo, Mimi Cho, Cha, Quamrul Hassan and many other former and recent members of the Stubbe group for spreading a good mood and giving me the temporary feeling to be part of.

I am grateful to all my group members who had a big contribution to my work. First of all Giuseppe alias Emir Kusturica, I only can wish that you stay forever on the scientific road. Therefore let's use one of your famous sentences: "You can do everything, if you want!" I can only add to this: "You too, and long live Orazio!"

Maria my PhD mate brought a bit of relaxation with her cool attitude into the group which sometimes could not be topped, even by our one and only Englishman and gentleman, Alistair. Thank you both for these rare moments.

"Igore, moj Igore!", thank you for tolerating my directness and sometimes unconventional style in saying my opinion. There were always delicate but also funny and honest moments between us.

And Irina, thank you for keeping the group alive in your natural way and for being one of the big protagonists of the cocktail tour through Göttingen. 
A big "thank you" goes to Gitta, the heart of our group who has for each difficulty the right solution. Roberta I like the funny and interesting conversations during the Italian coffee breaks and Soraya I appreciate your cool and reserved nature.

The newcomers Thomas, Nikolay, Roberto and Ines, I am sure you will make your way. I wish you good luck for your future projects and the will to succeed during your PhD.

I am grateful also to my collaborators Prof. Frank Neese and Christoph Riplinger who made a huge effort in performing the DFT calculations on the proton-coupled electron transfer pathway of E.coli RNR. Especially Christoph I would like to thank for his never-ending patience and the spontaneous and numerous telephone calls, visits, SMS, Skype contacts which finally ended up in very nice scientific results.

At the end my deepest gratitude goes to my family and my friends. The courage of my mother and father has no limits and they can be sure about the fact that they were right when they made the decision not to go back to Yugoslavia in the late 80 's. They made many sacrifices in their life and due to the long-sightedness of my mother I was able to get the best education available in Germany. My sister Zdenka, my brother-in-law Matthias and both nieces Sabrina and Sandra have been always and will be my big constants in life. There are no better, no funnier, no cooler nieces one can imagine to have. Thanks also to my openminded aunt Dana, my cousin Jasmin and aunt Lela, my cousin Helena and her family. Thanks to my friends Sebastian, Frank, Matthias, Sascha and their families. There are no excuses anymore to visit you. To my friends from the University of Frankfurt Senada, Katja and Anke, who are on the same track like me right now. To Julia and her "big" brother Jan, the trip over the ocean was great and hopefully soon there will be some more. To "Pistol Pete", who found the sun in Portugal. Ähm, your carpet got many compliments, I think I won't sell it. To the rest of my family back in Serbia, who will face the future for sure. And last but not least to the people, who were with me in their thoughts or who are still in my thoughts, thank you all!

Well, someone might say "it's just a PhD thesis" but I can't neglect anymore the obvious feeling that one very big circle is getting closed and another one will be opened soon. 


\section{Table of contents}

$\begin{array}{ll}\text { List of figures } & \text { page } 10\end{array}$

$\begin{array}{ll}\text { List of tables } & \text { page } 14\end{array}$

$\begin{array}{ll}\text { List of equations } & \text { page } 15\end{array}$

$\begin{array}{ll}\text { Abbreviations } & \text { page } 16\end{array}$

$\begin{array}{ll}\text { Abstract } & \text { page } 18\end{array}$

\section{Chapter 1 - General introduction}

1.1 Introduction to ribonucleotide reductase and their different classes

page 20

1.2 E.coli class la RNR

page 22

1.3 The radical propagation pathway in the E.coli class la RNR

page 25

1.3 Electron transfer in model proteins

page 28

1.4 Proton-coupled electron transfer (PCET) in RNR

page 32

1.5 Suppressor tRNA/aminoacyl-tRNA synthetase methodology

page 37

1.6 High-frequency EPR spectroscopy and the usage of the multifrequency approach

1.7 High-field ENDOR spectroscopy and the characterization of hydrogen interactions

page 52

Chapter 2 - Site-specific incorporation of 3-aminotyrosine into the E.coli RNR $\alpha$-subunit using an evolved suppressor tRNA/tRNA-synthetase pair

2.1 Introduction page 66

2.2 Results page 70

2.3 Discussion page 74

2.4 Materials and Methods page $75-79$ 
- Materials

page 75

- Preparation of LB plates for the transformation procedure

page 77

- Transformation of pTrc-nrdA $\mathrm{A}_{730 / 731} \mathrm{TAG}$ and $\mathrm{pAC}-\mathrm{NH}_{2} \mathrm{Y}-\mathrm{RS}$

into $\mathrm{DH} 10 \beta$ cells

page 77

- Column preparation for the purification of $\mathrm{NH}_{2} \mathrm{Y}_{730}-\alpha 2$ and $\mathrm{NH}_{2} \mathrm{Y}_{731}-\alpha 2$ page 77

- Preparation of SDS-gels for the analysis of purification and expression steps of $\mathrm{NH}_{2} \mathrm{Y}_{730}-\alpha 2$ and $\mathrm{NH}_{2} \mathrm{Y}_{731}-\alpha 2$

page 78

- Expression of $\mathrm{NH}_{2} \mathrm{Y}_{730}-\alpha 2$ and $\mathrm{NH}_{2} \mathrm{Y}_{731}-\alpha 2$ subunit page 78

- Purification of wt- $\alpha 2, \mathrm{NH}_{2} \mathrm{Y}_{730}-\alpha 2$ and $\mathrm{NH}_{2} \mathrm{Y}_{731}-\alpha 2$ subunit

page 79

\section{Chapter 3 - Multifrequency EPR characterization of 3-aminotyrosyl radicals on the radical propagation pathway of E.coli ribonucleotide reductase}

3.1 Introduction

page 81

3.2 Results

page 86-109

- Decomposition of $\mathrm{Y}_{122}{ }^{\circ}$ and $\alpha-\mathrm{ND}_{2} \mathrm{Y}_{730}{ }^{\circ}$ at $94 \mathrm{GHz}$ - Electron spin echo spectra at 70 and $10 \mathrm{~K}$

page 86

- Electron spin echo spectroscopy at $94 \mathrm{GHz}$ of $\alpha-\mathrm{NH}_{2} \mathrm{Y}_{730^{\circ}}$ and $\alpha-\mathrm{NH}_{2} \mathrm{Y}_{731}{ }^{\circ}$ in $\mathrm{H}_{2} \mathrm{O}$ and $\mathrm{D}_{2} \mathrm{O}$ assay buffer page 87

- Proton Davies ENDOR spectroscopy at $34 \mathrm{GHz}$ of $\alpha-\mathrm{NH}_{2} \mathrm{Y}_{730}{ }^{\circ}$ and $\alpha-\mathrm{NH}_{2} \mathrm{Y}_{731}{ }^{\circ}$ in $\mathrm{D}_{2} \mathrm{O}$ assay buffer page 91

- Spectral simulations of $\alpha-\mathrm{NH}_{2} \mathrm{Y}_{730^{\circ}}$ and $\alpha-\mathrm{NH}_{2} \mathrm{Y}_{731}{ }^{\circ}$ in $\mathrm{H}_{2} \mathrm{O}$ and

$\mathrm{D}_{2} \mathrm{O}$ assay buffer at 9 and $94 \mathrm{GHz}$

- Spectral simulations of $\beta-\mathrm{NH}_{2} \mathrm{Y}_{356}$ in $\mathrm{D}_{2} \mathrm{O}$ and $\mathrm{H}_{2} \mathrm{O}$ assay buffer 
at $94 \mathrm{GHz}$ and comparison of all $\mathrm{NH}_{2} \mathrm{Y}^{\bullet}$ on the radical propagation pathway

\section{Chapter 4 - High-field ENDOR/DFT characterization of hydrogen-bond} networks around $\alpha-\mathrm{NH}_{2} \mathrm{Y}_{730^{\circ}}$ and $\alpha-\mathrm{NH}_{2} \mathrm{Y}_{731}{ }^{\circ}$ in E.coli ribonucleotide reductase

- High-field ENDOR spectroscopy at $94 \mathrm{GHz}$ of $\alpha-\mathrm{NH}_{2} \mathrm{Y}_{730^{\circ}}$ and $\alpha-\mathrm{NH}_{2} \mathrm{Y}_{731}$ in deuterated buffer

- ENDOR spectroscopy at $34 \mathrm{GHz}$ of $\alpha-\mathrm{NH}_{2} \mathrm{Y}_{730^{\circ}}$ and $\alpha-\mathrm{NH}_{2} \mathrm{Y}_{731}{ }^{\bullet}$ in deuterated buffer

- Inspection of the $\alpha 2-\mathrm{NH}_{2} \mathrm{Y}_{730}$ crystal structure page 128

- Orientation selective high-field ENDOR on $\alpha-N_{2} \mathrm{Y}_{730^{\circ}}$ coupling to a distant deuteron

- High-field Mims ENDOR spectroscopy on $\operatorname{Tyr}_{122}{ }^{\bullet}$ in the resting state of the $\beta$-subunit compared with ENDOR spectroscopy on $\operatorname{Tyr}_{122}{ }^{\circ} / \alpha-N_{2} \mathrm{Y}_{730^{\circ}}$ in the active complex

- Orientation selective high-field ENDOR on $\alpha-\mathrm{ND}_{2} \mathrm{Y}_{730^{\circ}}$ coupling to external hydrogen-bonds

- Orientation selective high-field ENDOR on $\alpha-N_{2} \mathrm{Y}_{730^{\circ}}$ coupling to deuterons of the $\mathrm{ND}_{2}$ - group

- DFT calculations explain the experimental g-values and confirm hydrogen-bonds to the 3-aminotyrosyl radical in the protein environment

- Orientation selective high-field ENDOR on $\alpha-N_{2} \mathrm{Y}_{731}{ }^{\circ}$ coupling to a distant deuteron 
- Orientation selective high-field ENDOR on $\alpha-\mathrm{ND}_{2} \mathrm{Y}_{731}{ }^{\circ}$ coupling to external hydrogen-bonds

page 145

4.3 Discussion

page 148

\section{Materials and methods for chapter 3 and 4}

- Materials

page 154

- Preparation of high-field EPR samples

page 154

- Electron spin echo spectroscopy at 9, 34, 94, and $180 \mathrm{GHz}$

page 154

- Simulation of EPR spectra at 9, 34 and $94 \mathrm{GHz}$

page 155

- $34 \mathrm{GHz}$ Davies ENDOR spectroscopy on $\mathrm{NH}_{2} \mathrm{Y}_{730^{\circ}}$ and $\mathrm{NH}_{2} \mathrm{Y}_{731}{ }^{\circ}$

in $\mathrm{D}_{2} \mathrm{O}$ assay buffer

page 155

- 34 and $94 \mathrm{GHz}$ Mims ENDOR spectroscopy on $\alpha-\mathrm{NH}_{2} \mathrm{Y}_{730}{ }^{\circ}$ and $\alpha-\mathrm{NH}_{2} \mathrm{Y}_{731}$ in $\mathrm{D}_{2} \mathrm{O}$ buffer

page 156

- Simulation of ENDOR spectra

page 156

- DFT calculations

page 156

- Molecular visualization

page 158

Summary

page 159

References

page 165

Appendix

page 176

Curriculum vitae

page 185 


\section{List of figures}

Figure 1-1: 2'-deoxynucleotide formation in ribonucleotide reductase

Figure 1-2: Classes of ribonucleotide reductase

Figure 1-3: Classes Ib and Ic - subclasses of ribonucleotide reductase

Figure 1-4: E.coli RNR map

Figure 1-5: E.coli RNR docking model

Figure 1-6: The proposed radical transfer pathway of E.coli RNR

Figure 1-7: Chemical structure of $\mathrm{N}_{3}$ UDP

Figure 1-8: Distance distributions from PELDOR studies on the inactivated E.coli RNR

Figure 1-9: Possible models for radical pairs in the inactivated E.coli RNR

Figure 1-10: Timetable for electron tunneling through various media with respect to the donor-acceptor distance

Figure 1-11: Overlapping wave-functions for donor and acceptor at a fixed distance in electron transfer

Figure 1-12: The RNR PCET-model showing the long-range charge transfer over $35 \AA$

Figure 1-13: PCET model system for a symmetric dicarboxylate hydrogen-bond interface

Figure 1-14: Scheme for proton-coupled electron transfer

Figure 1-15: Hydrogen-bonded aminophenol that undergoes a concerted proton-electron transfer reaction

Figure 1-16: Scheme for two different PCET scenarios occurring in enzymes

Figure 1-17: Library of Methanococcus jannaschii TyrRS mutants

Figure 1-18: In vivo incorporation of $\mathrm{NH}_{2} \mathrm{Y}$ into the $\alpha$-subunit

Figure 1-19: Scheme for various magnetic interactions between radicals and their surrounding environment

Figure 1-20: Orientation selection by high-field EPR

Figure 1-21: Detection of two radical species at low and high frequencies

Figure 1-22: First derivative CW EPR spectra of $\mathrm{P}_{865}{ }^{\circ+}$ and $\mathrm{Q}_{A}{ }^{\circ-}$ for the frequencies 9.5, 95 and $360 \mathrm{GHz}$

Figure 1-23: Stimulated echo-detected EPR spectra at $140 \mathrm{GHz}$ of the E441Q RNR reaction

Figure 1-24: Hyperfine interaction between an electron and different nuclei 
Figure 1-25: Comparison of ENDOR resolution at different fields

Figure 1-26: Schematic energy level diagram for an electron with $S=1 / 2$ coupled to a nucleus with $\mathrm{I}=1 / 2$

Figure 1-27: Principles of ENDOR spectroscopy

Figure 1-28: Dipole-dipole interaction between an electron and a nucleus

Figure 1-29: Powder patterns for a hyperfine tensor $A$ with three different examples

Figure 1-30: The Mims ENDOR sequence

Figure 1-31: The Mims hole function

Figure 1-32: The Davies ENDOR sequence

Figure 1-33: Splitting pattern due to hyperfine and quadrupole interaction of an electron spin of $S=1 / 2$ with a nuclear spin of $I=1$

Figure 2-1: Proposed radical transfer pathway of E.coli RNR showing $\mathrm{NH}_{2} \mathrm{Y}$ substitutions of $Y_{730}$ and $Y_{731}$ in $\alpha 2$

Figure 2-2: One electron oxidation of $\mathrm{NH}_{2} \mathrm{Y}$

Figure 2-3: General scheme for selection of $\mathrm{NH}_{2} \mathrm{Y}-\mathrm{RS}$

Figure 2-4: SDS PAGE analysis of $\alpha 2$ expression and purification

Figure 2-5: DEAE column elution profile

Figure 2-6: SDS PAGE analysis of $\alpha 2$ expression and purification ( $2^{\text {nd }}$ part)

Figure 2-7: Representative SDS PAGE analysis of $\mathrm{NH}_{2} \mathrm{Y}_{730 / 731}-\alpha 2$ expression

Figure 2-8: Incubation of the $\mathrm{NH}_{2} \mathrm{Y}_{730}-\alpha 2: \beta 2$ complex with NDP's and NTP's

Figure 3-1: Multifrequency EPR spectra of $\alpha-\mathrm{NH}_{2} \mathrm{Y}_{730}{ }^{\circ}$ in the presence of CDP/ATP as a substrate/effector pair in $\mathrm{H}_{2} \mathrm{O}$ assay buffer

Figure 3-2: Structure of $\mathrm{NH}_{2} \mathrm{Y}_{730}$ 'showing the intramolecular hydrogen-bond

Figure 3-3: PyMol structure of $\mathrm{NH}_{2} \mathrm{Y}_{730}{ }^{\circ}$

Figure 3-4: $94 \mathrm{GHz}$ EPR spectroscopy on $\mathrm{Y}_{122}{ }^{\circ} / \mathrm{NH}_{2} \mathrm{Y}_{730}{ }^{\circ}$ and $\mathrm{NH}_{2} \mathrm{Y}_{730} \cdot$

Figure 3-5: Comparison of $94 \mathrm{GHz}$ pulsed derivative EPR spectra of $\mathrm{NH}_{2} \mathrm{Y}_{730}$ in $\mathrm{H}_{2} \mathrm{O}$ and $\mathrm{D}_{2} \mathrm{O}$ assay buffer

Figure 3-6: Comparison of $94 \mathrm{GHz}$ pulsed derivative EPR spectra of $\mathrm{NH}_{2} \mathrm{Y}_{731}$ in $\mathrm{H}_{2} \mathrm{O}$ and $\mathrm{D}_{2} \mathrm{O}$ assay buffer

Figure 3-7: Time course of the new radical species in the $\mathrm{NH}_{2} \mathrm{Y}_{731}{ }^{\circ}$ spectrum 
Figure 3-8: $34 \mathrm{GHz}{ }^{1} \mathrm{H}$ Davies ENDOR on $\alpha-\mathrm{NH}_{2} \mathrm{Y}_{730}{ }^{\circ}$ in $\mathrm{D}_{2} \mathrm{O}$ assay buffer

Figure 3-9: $34 \mathrm{GHz}{ }^{1} \mathrm{H}$ Davies ENDOR on $\alpha-\mathrm{NH}_{2} \mathrm{Y}_{731}{ }^{\bullet}$ in $\mathrm{D}_{2} \mathrm{O}$ assay buffer

Figure 3-10: Simulations of the first derivative EPR spectrum of $\mathrm{NH}_{2} \mathrm{Y}_{730}$ in $\mathrm{D}_{2} \mathrm{O}$ assay buffer at $9 \mathrm{GHz}$

Figure 3-11: Simulation of the first derivative EPR spectrum of $\mathrm{NH}_{2} \mathrm{Y}_{730}$ in $\mathrm{D}_{2} \mathrm{O}$ assay buffer at $94 \mathrm{GHz}$

Figure 3-12: Illustration of $A$ - and g-tensors and the Euler rotation

Figure 3-13: Simulations of the first derivative EPR spectrum of $\mathrm{NH}_{2} \mathrm{Y}_{730}$ in $\mathrm{H}_{2} \mathrm{O}$ assay buffer at $9 \mathrm{GHz}$

Figure 3-14: Simulation of the first derivative EPR spectrum of $\mathrm{NH}_{2} \mathrm{Y}_{730}$ in $\mathrm{H}_{2} \mathrm{O}$ assay buffer at $94 \mathrm{GHz}$

Figure 3-15: Simulation of the first derivative EPR spectrum of $\mathrm{NH}_{2} \mathrm{Y}_{731}$ in $\mathrm{D}_{2} \mathrm{O}$ assay buffer at $94 \mathrm{GHz}$

Figure 3-16: Simulation of the first derivative EPR spectrum of $\mathrm{NH}_{2} \mathrm{Y}_{731}$ in $\mathrm{H}_{2} \mathrm{O}$ assay buffer at $94 \mathrm{GHz}$

Figure 3-17: Simulation of the first derivative EPR spectrum of $\beta-\mathrm{NH}_{2} \mathrm{Y}_{356}{ }^{\bullet}$ in $\mathrm{D}_{2} \mathrm{O}$ assay buffer at $94 \mathrm{GHz}$

Figure 3-18: Simulation of the first derivative EPR spectrum of $\beta-\mathrm{NH}_{2} \mathrm{Y}_{356}{ }^{\bullet}$ in $\mathrm{H}_{2} \mathrm{O}$ assay buffer at $94 \mathrm{GHz}$

Figure 3-19: Comparison of all $1^{\text {st }}$ derivative $\mathrm{NH}_{2} \mathrm{Y}^{\bullet}$ spectra recorded in $\mathrm{D}_{2} \mathrm{O}$ buffer

Figure 3-20: Comparison of all $1^{\text {st }}$ derivative $\mathrm{NH}_{2} \mathrm{Y}^{\bullet}$ spectra recorded in $\mathrm{H}_{2} \mathrm{O}$ buffer

Figure 3-21: Euler rotation exemplified on the $\mathrm{N}-\mathrm{H}(2)$ bond

Figure 3-22: Euler rotation exemplified on the $\mathrm{N}-\mathrm{H}(1)$ bond

Figure 3-23: Definition of the dihedral angle and the angle $\Theta$

Figure 3-24: The two radicals present in the $\mathrm{NH}_{2} \mathrm{Y}_{730^{-}} \alpha 2: \beta 2$ complex after incubation with substrate and effector

Figure 3-25: Models for the $\mathrm{Y}_{731}-\mathrm{NH}_{2} \mathrm{Y}_{730}-\mathrm{C}_{439}$ pathway in $\mathrm{NH}_{2} \mathrm{Y}_{730}-\alpha 2$

Figure 3-26: Model A for the $\mathrm{Y}_{731}-\mathrm{NH}_{2} \mathrm{Y}_{730}-\mathrm{C}_{439}$ pathway in $\mathrm{NH}_{2} \mathrm{Y}_{730}-\alpha 2$

Figure 4-1: Crystal structure of the $\mathrm{NH}_{2} \mathrm{Y}_{730}-\alpha 2$ mutant of E.coli RNR 
Figure 4-2. Crystal structure of the $\mathrm{NH}_{2} \mathrm{Y}_{730^{-}} \alpha 2$ mutant of E.coli RNR in the flipped conformation

Figure 4-3. Crystal structure of the $\mathrm{NH}_{2} \mathrm{Y}_{730}-\alpha 2$ mutant of E.coli RNR in the flipped conformation co-incubated with a 20-mer tail of the $\beta$-subunit

Figure 4-4. Crystal structure of the $\mathrm{NH}_{2} \mathrm{Y}_{731}-\alpha 2$ mutant of E.coli RNR showing two different conformations of $\mathrm{NH}_{2} \mathrm{Y}_{731}$

Figure 4-5: $94 \mathrm{GHz}^{2} \mathrm{H}$ Mims ENDOR spectrum of $\mathrm{NH}_{2} \mathrm{Y}_{730}{ }^{\circ}$ in $\mathrm{D}_{2} \mathrm{O}$ assay buffer

Figure 4-6: $94 \mathrm{GHz}^{2} \mathrm{H}$ Mims ENDOR spectrum of $\mathrm{NH}_{2} \mathrm{Y}_{731}$ in $\mathrm{D}_{2} \mathrm{O}$ assay buffer

Figure 4-7: Comparison of both $\mathrm{NH}_{2} \mathrm{Ys}^{\circ}$ in $\mathrm{D}_{2} \mathrm{O}$ assay buffer which were recorded under the same experimental ENDOR conditions

Figure 4-8: Comparison of two $\mathrm{ND}_{2} \mathrm{Y}_{730^{\circ}}$ ENDOR spectra measured with two different $\tau$ values

Figure 4-9: Demonstrative example of the excitation bandwidth of a $\pi / 2$ pulse at $W$-band and Q-band frequency

Figure 4-10: Comparison of $\mathrm{ND}_{2} \mathrm{Y}_{730^{\circ}}$ and $\mathrm{ND}_{2} \mathrm{Y}_{731}{ }^{\circ}$ ENDOR spectra measured at Q-band frequency

Figure 4-11: Crystal structure of $\mathrm{NH}_{2} \mathrm{Y}_{730}-\alpha 2$ together with hydrogens which could be potentially observed during activity in a high-field ENDOR experiment

Figure 4-12: Orientation selective ${ }^{2} \mathrm{H}$ Mims ENDOR spectra of $\mathrm{NH}_{2} \mathrm{Y}_{730}{ }^{\circ}$ in $\mathrm{D}_{2} \mathrm{O}$ assay buffer of the weakly coupled region

Figure 4-13. ${ }^{2} \mathrm{H}$ Mims ENDOR spectroscopy of $\alpha-\mathrm{NH}_{2} \mathrm{Y}_{730} / \beta-\mathrm{Y}_{122}{ }^{\bullet}$ in the active complex and of $Y_{122}{ }^{\bullet}$ in the resting state of the $\beta$-subunit in $D_{2} \mathrm{O}$ buffer

Figure 4-14: Orientation selective ${ }^{2} \mathrm{H}$ Mims ENDOR spectra of $\mathrm{NH}_{2} \mathrm{Y}_{730^{\circ}}$ in $\mathrm{D}_{2} \mathrm{O}$ assay buffer in the region $\pm 1.5 \mathrm{MHz}$ where external hydrogen bonds are expected

Figure 4-15: Orientation selective ${ }^{2} \mathrm{H}$ Mims ENDOR spectra of $\mathrm{NH}_{2} \mathrm{Y}_{730}{ }^{\bullet}$ in $\mathrm{D}_{2} \mathrm{O}$ assay buffer of the whole spectral region

Figure 4-16: Summary of six calculated DFT cluster models

Figure 4-17: Combined EPR and DFT model for the hydrogen-bond network around $\mathrm{NH}_{2} \mathrm{Y}_{730}$ • in the $\alpha$-subunit

Figure 4-18: Orientation selective ${ }^{2} \mathrm{H}$ Mims ENDOR spectra of $\mathrm{NH}_{2} \mathrm{Y}_{731}$ in $\mathrm{D}_{2} \mathrm{O}$ assay buffer of the weakly coupled region 
Figure 4-19: Orientation selective ${ }^{2} \mathrm{H}$ Mims ENDOR spectra of $\mathrm{NH}_{2} \mathrm{Y}_{731}{ }^{\bullet}$ in $\mathrm{D}_{2} \mathrm{O}$ assay buffer in the region $\pm 1.5 \mathrm{MHz}$ where external hydrogen bonds are expected

Figure 4-20: Crystal structure of $\mathrm{NH}_{2} \mathrm{Y}_{731}-\alpha 2$ and locations of deuterons obtained from the analysis of the orientation selective ENDOR spectra of $\alpha-\mathrm{NH}_{2} \mathrm{Y}_{731}{ }^{\bullet}$ in $\mathrm{D}_{2} \mathrm{O}$ assay buffer

Figure 4-21. Energy level diagram including the transition states for the PCET pathway residues $Y_{731}-Y_{730}-C_{439}$

\section{List of Tables}

Table 1-1: Commonly used frequency bands in EPR spectrometers

Table 1-2: Summary of principal g-values and g-anisotropy for various tyrosyl radicals from different class I RNR enzymes

Table 2-1: Summarized cell amounts and induction parameters for ten expressions of $\mathrm{NH}_{2} \mathrm{Y}_{730 / 731-\alpha}$

Table 3-1: Summary of all EPR parameters which were used in the simulation of $\mathrm{NH}_{2} \mathrm{Y}^{\bullet} \mathrm{s}$ in $\mathrm{D}_{2} \mathrm{O}$ and $\mathrm{H}_{2} \mathrm{O}$ buffer at position $\beta-356, \alpha-731$ and $\alpha-730$ on the radical propagation pathway of E.coli RNR

Table 4-1: Parameter set for deuterons which are a constituent of the external hydrogenbond network and part of the 3-aminotyrosyl radical at position 730

Table 4-2: Summarized g-values from small model systems (DFT calculations) and from experiment

Table 4-3: Summary of g-values resulting from calculations of six DFT cluster models together with model features

Table 4-4: Parameter set for deuterons around the 3-aminotyrosyl radical at position 731 


\section{List of Equations}

Equation 1-1: The Marcus-Levich equation describing ET in chemical and biological systems Equation 1-2: Expression for electronic coupling

Equation 1-3: Kinetic expression for consecutive electron and proton transfer

Equation 1-4: Kinetic expression for concerted proton-coupled electron transfer in hydrogen-bonded aminophenols

Equation 1-5: Spin Hamiltonian for an electron spin coupled to a nucleus $(S=1 / 2$ and $I=1 / 2$ ) Equation 1-6: The hyperfine interaction expressed as a sum of a scalar and a tensor in the laboratory frame

Equation 1-7: Diagonalized dipolar tensor with its principal values $T_{\|}$and $T_{\perp}$

Equation 1-8: Point-dipole model formula for the calculation of interspin distances

Equation 1-9: Quadrupole Hamiltonian describing the quadrupole interaction

Equation 4-1: Point-dipole model formula considering the dipole interaction between one nucleus and the electron spin density of the oxygen of $\mathrm{NH}_{2} \mathrm{Y}_{730}{ }^{\circ}$ 


\section{Abbreviations}

\begin{tabular}{|c|c|}
\hline A & adenine \\
\hline ADP & adenosine-5'-diphosphate \\
\hline ATP & adenosine-5'-triphosphate \\
\hline CDP & cytidine-5'-diphosphate \\
\hline C & cytosine \\
\hline $\mathrm{CW}$ & continuous wave \\
\hline CV & column volumes \\
\hline dATP & deoxyadenosine triphosphate \\
\hline dGTP & deoxyguanosine triphosphate \\
\hline DFT & Density Functional Theory \\
\hline DOPA & 3,4-dihydroxyphenylalanin \\
\hline diag & diagonalized \\
\hline ENDOR & Electron Nuclear Double Resonance \\
\hline EPR & Electron Paramagnetic Resonance \\
\hline ESI & Electrospray Ionisation \\
\hline ET & electron transfer \\
\hline G & guanine \\
\hline GDP & guanosine-5'-diphosphate \\
\hline $\mathrm{h}$ & hours \\
\hline HAT & hydrogen atom transfer \\
\hline hf & high-field \\
\hline
\end{tabular}




\begin{tabular}{|c|c|}
\hline INDO & Intermediate Neglect of Differential Overlap \\
\hline LB & Luria Bertani \\
\hline MALDI-TOF & Matrix Assisted Laser Desorption Ionisation - Time of Flight \\
\hline MTSL & methanethiosulfonate \\
\hline MW & microwave \\
\hline NADPH & $\beta$-nicotinamide adenine dinucleotide phosphate \\
\hline NMR & Nuclear Magnetic Resonance \\
\hline $\mathrm{OD}_{600}$ & optical density at $600 \mathrm{~nm}$ \\
\hline PCET & proton-coupled electron transfer \\
\hline PDB ID & Protein database identification code \\
\hline PELDOR & Pulsed Electron-Electron Double Resonance \\
\hline $\mathrm{RF}$ & radio frequency \\
\hline RNR & ribonucleotide reductase \\
\hline SDS-PAGE & Sodium Dodecylsulfate Polyacrylamide Gel Electrophoresis \\
\hline $\mathrm{sec}$ & seconds \\
\hline SOC & super optimal broth with added glucose \\
\hline TTP & thymidine triphosphate \\
\hline U & uracil \\
\hline UDP & uridine- $5^{\prime}$-diphosphate \\
\hline wt & wild type \\
\hline
\end{tabular}




\title{
High-field EPR and ENDOR spectroscopy for proton-coupled electron transfer investigations in E.coli ribonucleotide reductase
}

\begin{abstract}
Ribonucleotide reductases (RNRs) catalyze the conversion of nucleotides to deoxynucleotides in all organisms, thereby providing the essential precursors for DNA synthesis and replication. The E.coli RNR is composed of two homodimeric subunits: $\alpha 2$ and $\beta 2$. In $\alpha 2$ nucleotides are converted to deoxynucleotides and $\beta 2$ contains a stable di-iron tyrosyl radical $\left(\mathrm{Y}_{122}{ }^{\circ}\right)$ cofactor. A proton-coupled electron transfer (PCET) mechanism is proposed to occur over a distance of more than $35 \AA$ originating from $\mathrm{Y}_{122}$ and ending up in the generation of a $\mathrm{C}_{439}{ }^{\circ}$ in $\alpha 2$ which in turn initiates the nucleotide reduction process. Three conserved redox-active tyrosines $\left(\beta 2-Y_{356}, \alpha 2-Y_{730}\right.$ and $\left.\alpha 2-Y_{731}\right)$ are essential for the radical transfer to $\mathrm{Cys}_{439}$ and are proposed to be connected through hydrogenbonds during reversible charge migration over both subunits. In order to probe for structural and mechanistic requirements of a PCET event in $\alpha 2, \mathrm{NH}_{2} \mathrm{Y}$ has been incorporated through the suppressor tRNA/aminoacyl-tRNAsynthetase methodology at positions 730 and 731 , respectively. $\mathrm{NH}_{2} \mathrm{Y}$ functions hereby as a radical spin probe on the PCET pathway and allows the elucidation of chemical environments through high-field EPR and electron-nuclear double resonance (ENDOR) spectroscopy. In order to characterize the generated 3-aminotyrosyl radical $\left(\mathrm{NH}_{2} \mathrm{Y}^{\circ}\right)$ in $\mathrm{D}_{2} \mathrm{O}$ and $\mathrm{H}_{2} \mathrm{O}$ buffer, multifrequency EPR spectroscopy was first applied on $\mathrm{NH}_{2} \mathrm{Y}_{730^{\circ}}$. The characterization of the electronic and molecular structure of a $\mathrm{NH}_{2} \mathrm{Y}^{\bullet}$ was a crucial step in order to deconvolute the hyperfine couplings of internal protons from external protons which build up a hydrogen-bond network around $\alpha-\mathrm{NH}_{2} \mathrm{Y}_{730^{\circ}}$. High-field ENDOR spectroscopy detected two hydrogen-bonds which point into the direction of the next PCET pathway residues and additionally a water molecule which is placed near $\alpha-\mathrm{NH}_{2} \mathrm{Y}_{730^{\circ}}$ and could have mechanistic functions during the proposed $\mathrm{H}$-atom transfer process. $\mathrm{A}$ similar hydrogen-bond network was detected for $\alpha-\mathrm{NH}_{2} \mathrm{Y}_{731}{ }^{\circ}$ with a water molecule placed near the $\alpha / \beta$ interface. Recent DFT calculations on the PCET mechanism in $\alpha 2$ are hereby in agreement with structural insights obtained from high-field EPR and ENDOR studies.
\end{abstract}


Chapter 1

General introduction 


\subsection{Introduction to ribonucleotide reductase and their different classes}

Ribonucleotide reductases (RNR's) catalyze the conversion of nucleotides into their corresponding deoxynucleotides in all organisms and play thereby a central role in the synthesis of the monomeric precursors required for DNA replication and repair. The hydroxyl group at the 2' position of the nucleotides $C, G, U$ and $A$ gets hereby replaced through a hydrogen atom through a remarkably efficient and complex radical reaction mechanism $(1,2)$. A thiyl radical $\left(C^{\circ}\right)$ initiates hereby the catalytic process through the abstraction of a hydrogen atom at the $\mathrm{C3}^{\prime}$ position of the sugar moiety (Figure 1-1). The mentioned abstraction mechanism is common and accepted for all RNRs but will not be discussed here in this Thesis. Extensive work has been performed on the nucleotide reduction mechanism and original papers and reviews give a detailed explanation on this unprecedented radical reaction (3-6).

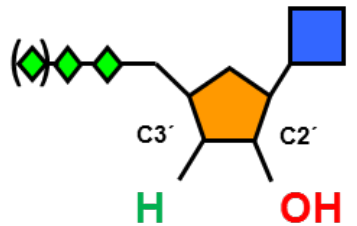

Nucleotides: C,G,U,A

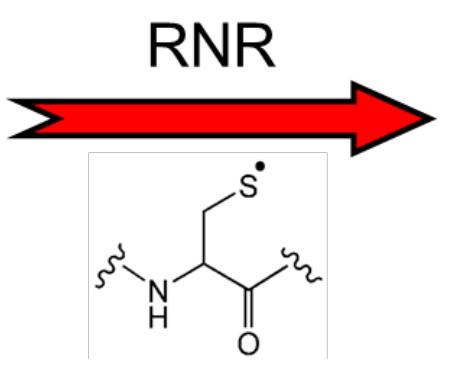

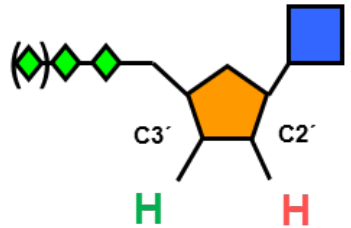

Deoxynucleotides: $\mathrm{dC}, \mathrm{dG}, \mathrm{dU}, \mathrm{dA}$

Figure 1-1. 2'-deoxynucleotide formation initiated through abstraction of a C3' hydrogen by a RNR thiyl radical. Phosphate groups are shown in green, the pentose sugar in orange and the base in blue. Other atoms or functional groups are omitted for clarity.

RNR's have been proposed to be the major link between RNA and DNA world. Although RNA's possess the ability to function as bio-catalysts it was proposed that they would not be able to control free radical chemistry and to synthesize deoxynucleotides. Therefore it is suggested that RNR's performed the nucleotide reduction process in a shielding environment and that they appeared during evolution before DNA $(7,8)$. Whereas the activation of the ribose by $\mathrm{a}^{\bullet}$ for the removal of the $\mathrm{OH}$-group is identical for all RNR enzymes, major differences appear in the generation of the $C^{\bullet}$. Three classes have been proposed for radical generation until now based on different metallocofactors which arose $\mathbf{2 0} \mid \mathrm{P}$ a g e 
by divergent evolution from a common RNR ancestor (Figure 1-2). Class I RNR's use a diiron tyrosyl radical $\left(\mathrm{Y}^{\bullet}\right)$ cofactor, class II a $5^{\prime}$-deoxyadenosyl radical derived from homolytic cleavage of the C-Co bond in adenosylcobalamin and class III RNR's employ a glycyl radical $\left(G^{\circ}\right)$ which is generated by an activase, in a reaction involving S-adenosylmethionine and a reduced iron-sulphur centre. Like for all class II RNR's a 5'-deoxyadenosyl radical is formed but this time through reductive cleavage of the $\mathrm{C}-\mathrm{S}^{+}$bond (9).

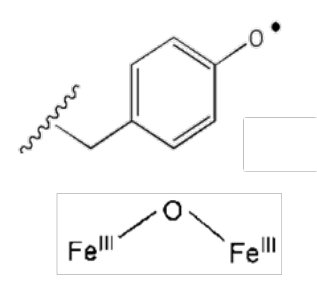

\section{class I}
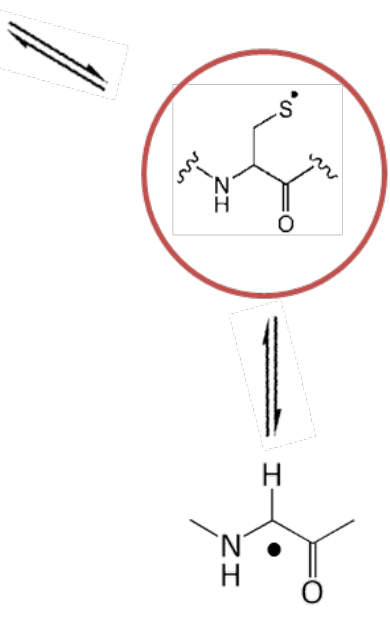

class III

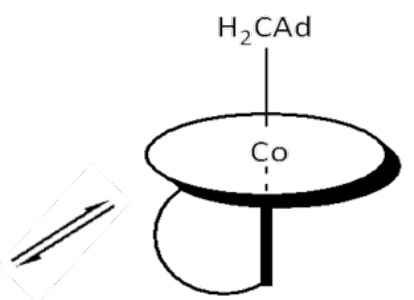

class II

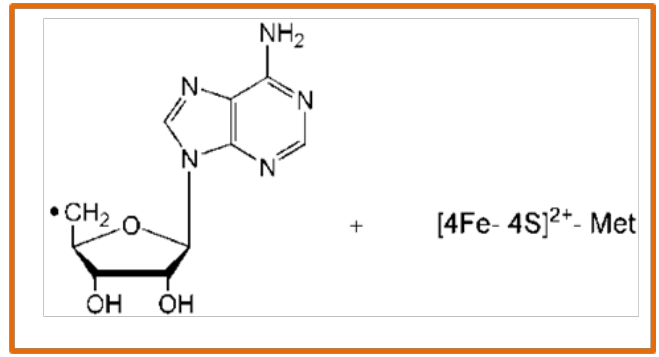

activase

Figure 1-2. Three classes of RNR are proposed to initiate the essential thiyl radical based on different metallocofactors. The activation of the class I RNR cofactor occurs through $\mathrm{O}_{2}$ whereas in class II RNR it occurs in situ under aerobic and anaerobic conditions. In case of class III RNR an additional enzyme generates the glycyl radical cofactor.

Only recently two new subclasses of class I RNR have been identified with both containing manganese in the metallocofactor site (Figure 1-3). The characterization of the dimanganese cofactor in class Ib RNR was reported through X- and Q-band EPR spectroscopy and X-ray crystallography and resembles the di-iron tyrosyl cofactor of class I RNR from E.coli (10-12). Class Ic RNR however lacks the well-known tyrosyl radical and is proposed to generate the $C^{\bullet}$ directly through the stable manganese (IV)/iron (III) cofactor (13). 


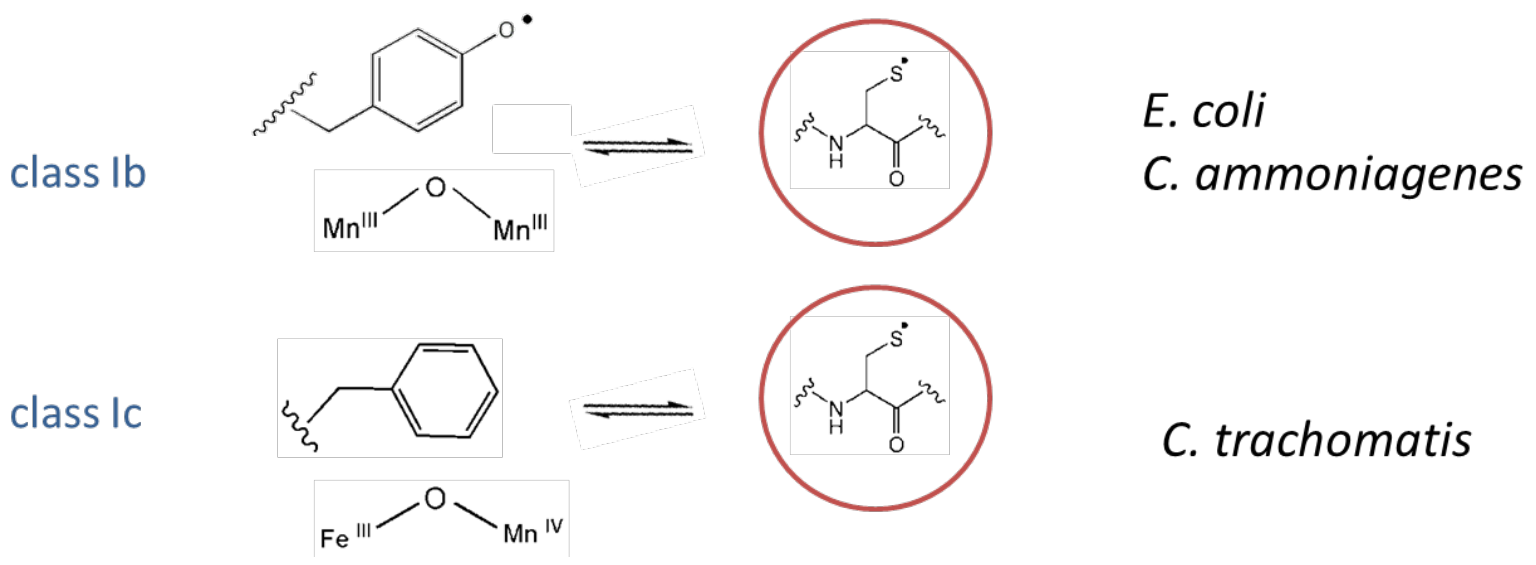

Figure 1-3. Class Ib and Ic are subclasses of class I. The recently investigated class Ib radical cofactor is most probably generated through a peroxide species which is formed through the reduction of $\mathrm{O}_{2}$ and an additional FMN containing enzyme called flavodoxin Nrdl (not shown).

For the awareness of the reader the literature for the Thesis has been checked until August 2011.

\subsection{E.coli class la RNR}

The E.coli class la RNR is composed of two homodimeric protein subunits, $\alpha 2$ and $\beta 2$ (Figure 1-4). In $\alpha 2$ the nucleotides CDP, GDP, UDP and ADP are converted into their corresponding deoxy-form. In order to provide a balanced pool for all four deoxynucleotides the E.coli RNR uses a unique allosteric regulation mechanism with ATP, dATP, TTP and dGTP as effectors which define the NDP to dNDP conversion. The structural basis for this regulation has so far been studied for different classes of RNR besides class la, leading to a general picture where binding of effectors in the dimer interface of $\alpha 2$ cause a conformational change which reaches the active site and determines which substrate gets reduced (14-17). An additional allosteric regulation site in class la enzymes is the activity site, which controls the overall rate of nucleotide reduction. Low [dATP] and high [ATP] stimulate nucleotide reduction whereas high [dATP] slows down the enzymatic activity of RNR (18). 


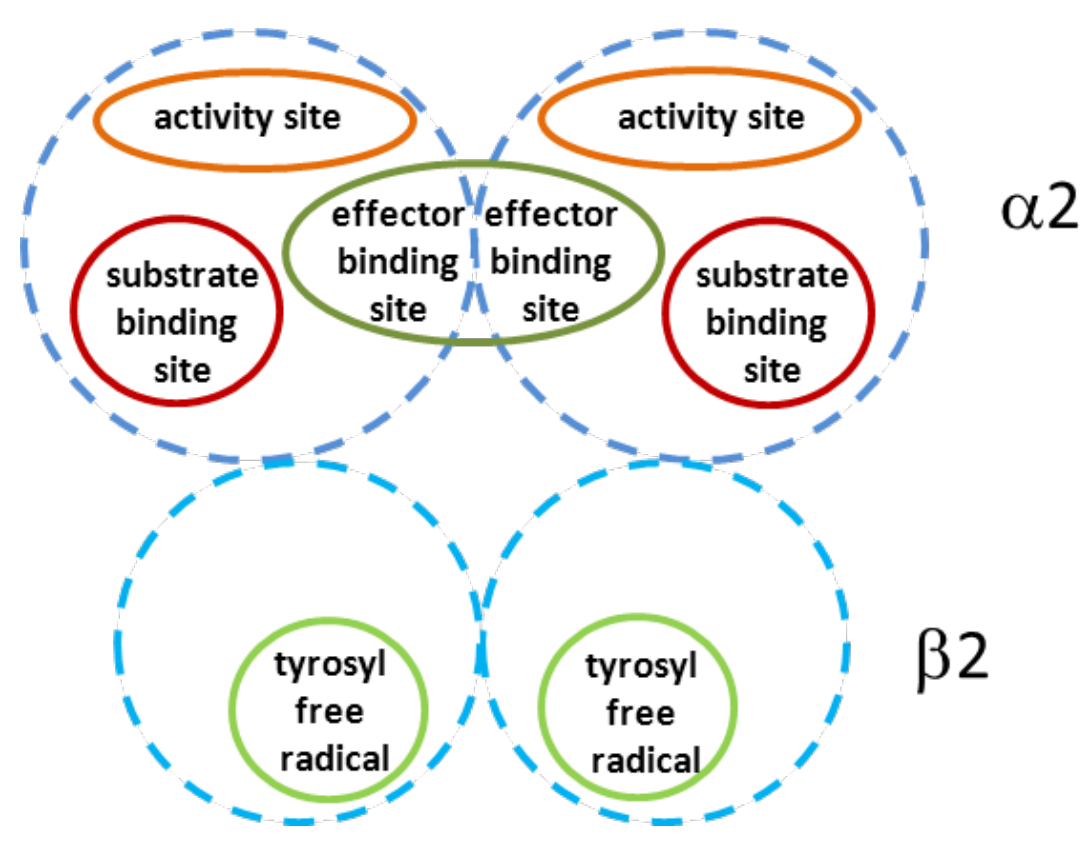

Figure 1-4. E.coli RNR map showing a schematic representation of important protein locations in the two homodimeric subunits $\alpha 2$ and $\beta 2$ which are necessary for enzymatic function, specificity and regulation.

After the discovery of the E.coli RNR in the early 1960s more than a decade has passed until the detection of an EPR signal which originates from the tyrosyl free radical in the $\beta 2$ subunit at location 122. The signal is dependent on the presence of iron and linked to the activity of the enzyme (19). Since then many other enzymes containing tyrosyl or other amino acid radicals could be characterized like the thiyl radical ( $C^{\circ}$ ) from RNR class II (20), the glycyl radical from pyruvate formate lyase which is also present in RNR class III $(21,22)$ and the tryptophan radical from cytochrome peroxidase (23).

From all RNR's the E.coli class I enzyme is to date the best studied RNR in the field of biochemistry as well as physical and theoretical chemistry. Numerous studies have been performed on the detailed characterization of the persistent $\mathrm{Y}_{122}{ }^{\bullet}$ through EPR and ENDOR methods (24) (25), on the assembly of the di-iron cluster (26-29), and on substrate-derived radical and protein radical intermediates (30-32) which could verify specific steps of the nucleotide reduction mechanism proposed by Stubbe and co-workers in the early 90 's.

Initial studies using ultracentrifugation showed that $\alpha 2$ and $\beta 2$ form a 1:1 complex in the presence of ATP, TTP and dATP (33). However crystal structures of $\beta 2$ and $\alpha 2$ appeared primarily in the early 90's together with a docking model of the $\alpha 2: \beta 2$ complex which is 
based on shape and charge complementarity of both subunits (Figure 1-5) (34, 35). One of the most astonishing insights from the docking model is that the $Y_{122}{ }^{\bullet}$ in $\beta 2$ has to oxidize over more than $35 \AA$ the Cys $_{439}$ residue in $\alpha 2$. Such a long electron distance was proposed to be highly unlikely in order to account for the activity of the enzyme and it was initially thought that a significant conformational change of the two subunits has to occur. An additional feature of both subunits is that the C-terminal tails of $\beta 2$ (residues 345 - 375) and $\alpha 2$ (residues $738-761$ ) are not resolved in any of the structures. The tails are highly flexible and are responsible for $\alpha 2 / \beta 2$ subunit interaction. It is interesting that for the crystallization of $\alpha 2$, a 21 mer peptide which is identical to one part of the C-terminal tail (residues 355375 ) is needed to be co-incubated with $\alpha 2$. However a structure of the $\alpha 2: \beta 2$ complex like it is proposed during activity is still missing in order to reevaluate the docking model.

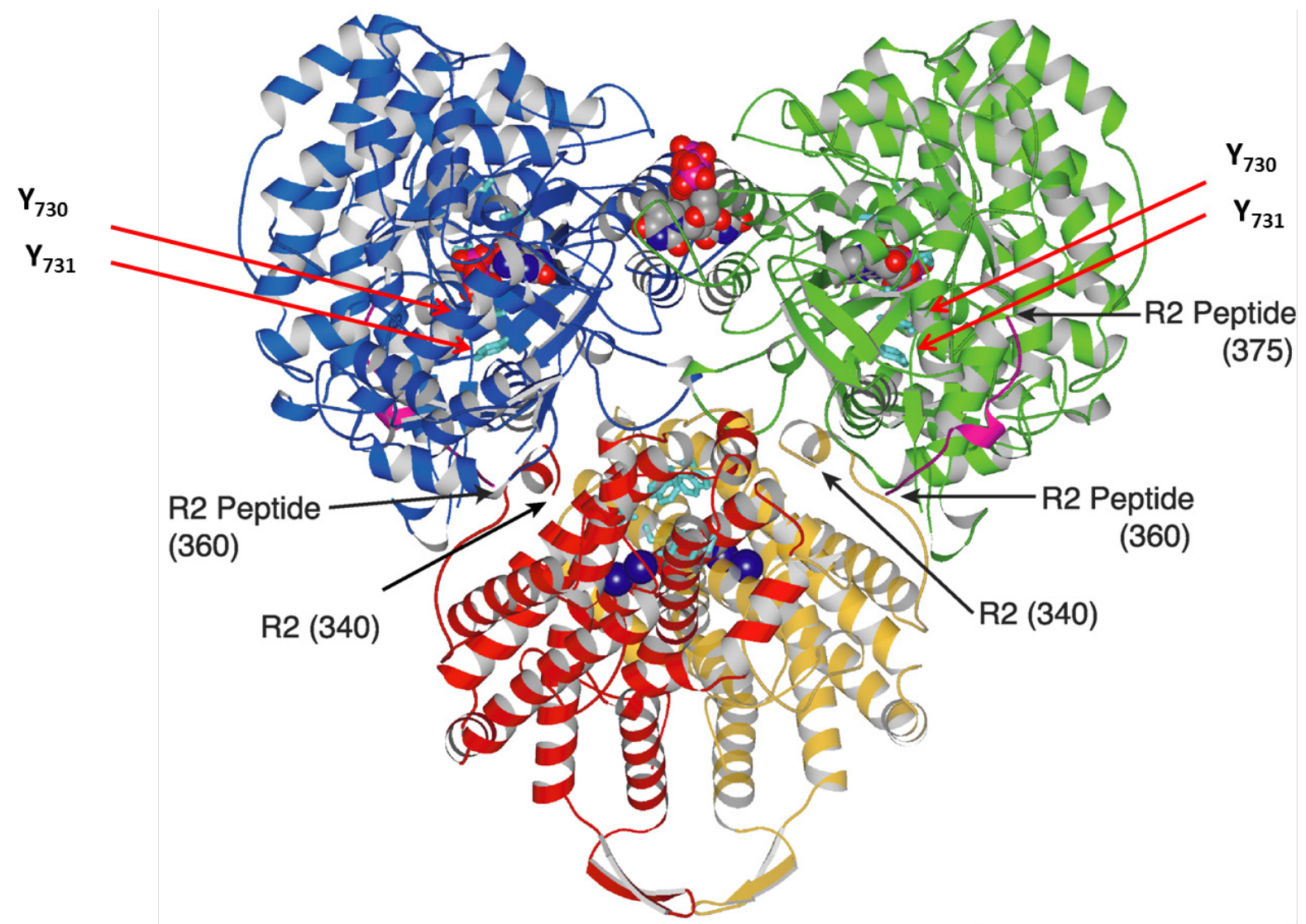

Figure 1-5. Docking model for the subunit interaction between $\alpha 2$ and $\beta 2$. The model was generated based on shape and charge complementarity between the two subunits. The monomers of $\alpha 2$ are shown in blue and green with substrate GDP bound in the substrate binding site and effector TTP at 
the effector binding site (shown as spheres). Each monomer contains a 21 mer peptide (shown in pink) consisting of the C-terminal residues of $\beta 2(355-375)$. In this structure only the residues $360-$ 375 are visible. Furthermore residues $Y_{730}$ and $Y_{731}$ of $\alpha 2$ are shown in light blue (see red arrows). The monomers of $\beta 2$ are shown in red and orange with the Fe atoms of the diiron cluster shown as dark blue spheres. In this structure the residues $Y_{122}, W_{48}$ and $D_{237}$ are shown in light blue. Residue 340 is the last residue that is observed in this structure with the residues 341-359 being missing in $\beta 2$ as well as residues 733-762 in $\alpha 2$.

\subsection{The radical propagation pathway in the E.coli class la RNR}

Based on the docking model a radical propagation pathway for RNR has been proposed which involves conserved residues in $\alpha 2$ and $\beta 2$ (Figure 1-6). Besides $Y_{122}$ and $C_{439}$ as the starting and end point of radical propagation $W_{48}$ and three $Y^{\prime} s, Y_{356}$ in $\beta 2$ and $Y_{730} / Y_{731}$ in $\alpha 2$ play a major role in charge transport. In comparison to other conserved residues the function of the three $Y^{\prime}$ 's which reversibly propagate the radical across the subunit interface from $\beta 2$ to $\alpha 2$ is not clear and is currently under detailed investigation.

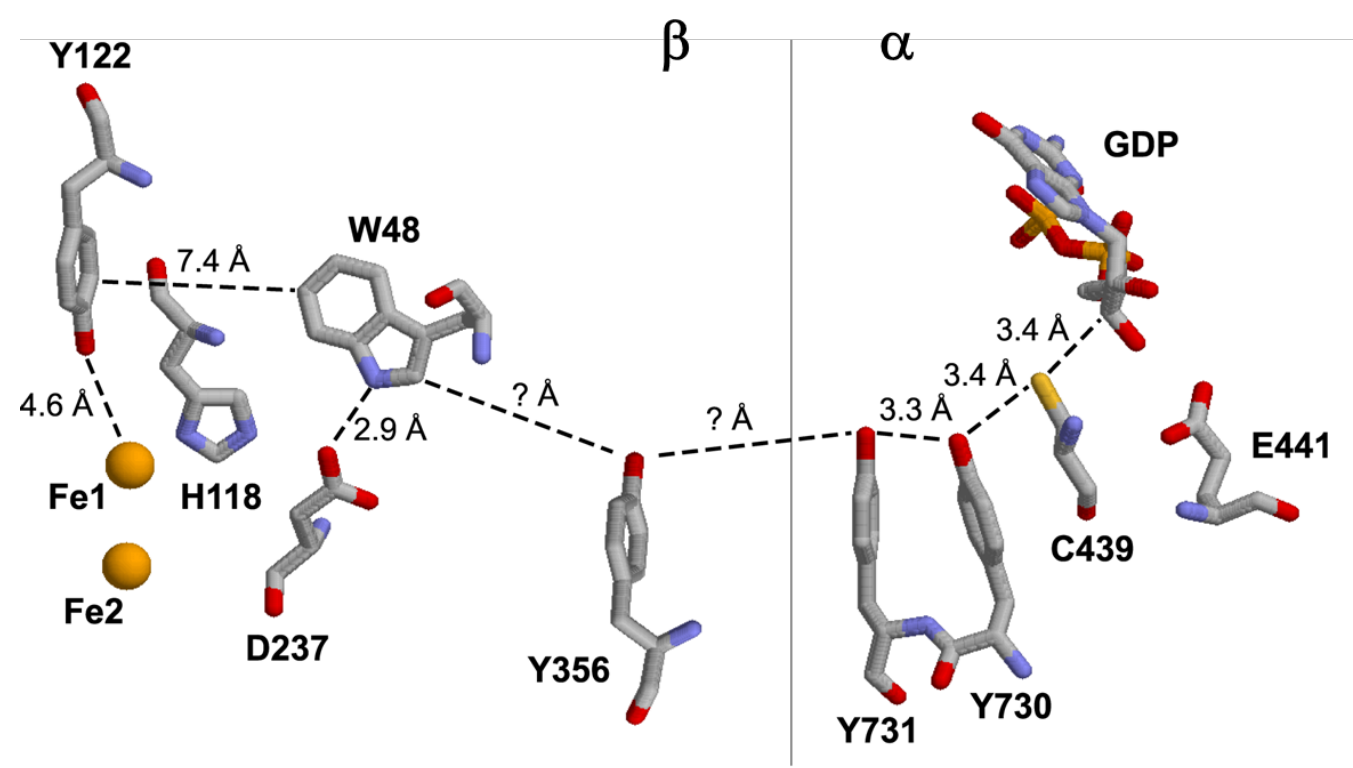

Figure 1-6. The proposed radical transfer pathway of E.coli RNR. Note that $Y_{356}$ from the $\beta$-subunit is not observable in any of the crystal structures and its distance to $W_{48}$ in the $\beta$-subunit and $Y_{731}$ in the $\alpha$-subunit is unknown. Other distances result from the two crystal structures of $\beta 2$ and $\alpha 2$. The distance between $W_{48}$ in $\beta 2$ and $Y_{731}$ in $\alpha 2$ is $25 \AA$. 
In no other biological systems are amino acids known to catalyze charge transport over such a long distance. A similar system involving radical propagation but over a much shorter distance than in RNR is DNA photolyase which uses three conserved W's for radical transport and the cofactor $\operatorname{FADH}(36,37)$. The distance from the cofactor to the last $W\left(W_{306}\right)$ is $13.3 \AA$. In comparison to many other redox-active enzymes like respiratory complex I, photosystem II and nitrogenase, RNR does not use any metals during radical propagation (38-40).

First attempts for testing the radical propagation pathway in RNR have been carried out through site-directed mutagenesis of each residue. The $\mathrm{Y}_{356} \mathrm{~F}-\beta 2$ mutant have been found to be inactive (41) and in addition in vivo studies demonstrated that this residue is necessary for catalysis inside the cell (42). Also the $Y_{730} F-\alpha 2$ and $Y_{731} F-\alpha 2$ mutants have been generated and the results showed no activity in vitro and in vivo. X-ray crystallography evidenced additionally no structural changes after phenylalanine substitutions of the pathway positions so that no perturbation of the binding to $\beta 2$ was expected (43). Although site-directed mutagenesis studies have proven the importance of each residue they were based on inactive mutants. Still from these and other theoretical studies, a chemical mechanism has been suggested which involves a series of hydrogen transfer steps between $Y_{122}$ in $\alpha 2$ and $\mathrm{C}_{439}$ in $\beta 2$ (44).

A slow conformational change that precedes rapid radical transfer is responsible for the lack of build-up of any observable intermediates which can be observed with stopped-flow UVvis or rapid-freeze-quench EPR spectroscopy (45). However the conformational change does not affect the distance between $Y_{122}{ }^{\circ}$ in $\beta 2$ and $C_{439}$ in $\alpha 2$ as it is evidenced through experiments with the mechanism-based inhibitor 2'-azido-2'-deoxyuridine-5'-diphosphate ( $\mathrm{N}_{3} \mathrm{UDP}$ ) (Figure 1-7) and concomitant pulsed electron-electron double resonance (PELDOR) spectroscopy, a method for determining distances between two or more spins which are separated $15-80 \AA(46,47)$. 


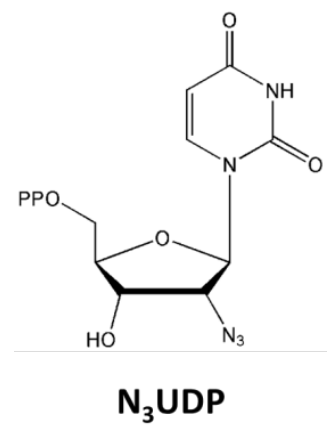

Figure 1-7. Chemical structure of $\mathrm{N}_{3}$ UDP which revealed the involvement of nucleotide radicals in the reduction process.

The reaction of RNR with $\mathrm{N}_{3}$ UDP results in destruction of the $\mathrm{Y}_{122} \cdot$ and formation of a new radical $\left(\mathrm{N}^{\circ}\right)$ in the active site of $\alpha 2(48)$. The structure of the $\mathrm{N}^{*}$ has been revealed through multifrequency EPR experiments along with DFT calculations. PELDOR spectroscopy has been used to measure the distance between $\mathrm{N}^{\bullet}$ generated with $\mathrm{N}_{3}$ UDP in the active site of $\alpha 2$ and the remaining $Y_{122}$ in $\beta 2$ (30). From the PELDOR studies a conformational change of both subunits could be excluded and the $\mathrm{N}^{\bullet}-\mathrm{Y}_{122}{ }^{\bullet}$ distance of $48.2 \AA$ which is derived from the docking model could be confirmed. Additionally a minor peak in the resulting distance distribution shows the $33 \AA$ distance between the two tyrosyl radicals in the $\beta 2$ subunit $\left(\mathrm{Y}_{122}{ }^{\bullet}\right.$ $-\mathrm{Y}_{122^{\circ}}$ ) (Figure 1-8).

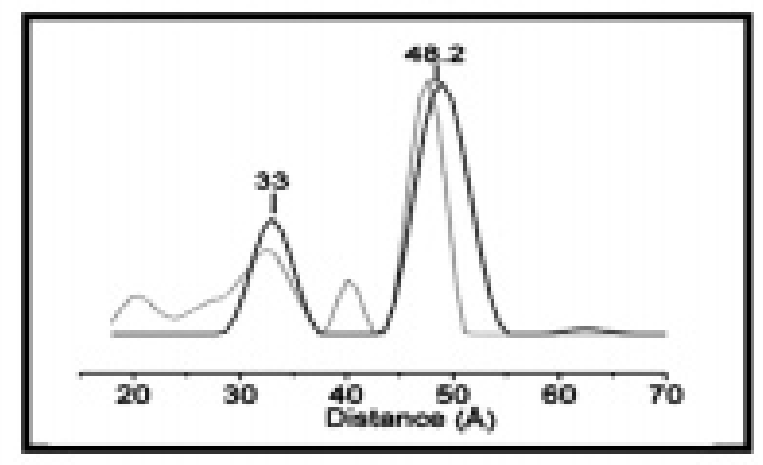

Figure 1-8.

Resulting distance distributions from PELDOR studies on the inactivated E.coli RNR with the $\mathrm{N}^{\bullet}-\mathrm{Y}_{122}{ }^{\bullet}$ cross distance of $48.2 \AA$ and the residual $33 \AA$ distance between $Y_{122}{ }^{\circ}-Y_{122}$ in $\beta 2$ (black trace). 
In order to exclude a possible distance of $48 \AA$ A between $\mathrm{N}^{\bullet}-\mathrm{N}^{\bullet}$ distance measurements were performed with the DQC (double-quantum coherence) method at $80 \mathrm{~K}$. At this temperature the $\mathrm{Y}_{122}{ }^{\bullet}$ signal can not be detected due to its shorter $\mathrm{T}_{2}$ relaxation time in comparison to the $\mathrm{N}^{\bullet}$ and the only signal which is left originates from the latter radical. In sum from the three different paramagnetic pairs: $\mathrm{Y}_{122}{ }^{\bullet}-\mathrm{Y}_{122}, \mathrm{Y}_{122}{ }^{\bullet}-\mathrm{N}^{\bullet}, \mathrm{N}^{\bullet}-\mathrm{N}^{\bullet}$ the first two could be confirmed and the last could be excluded (see Figure 1-9). The first distance measurements on the active complex support therefore a long-range radical transfer as well as the docking model. Moreover they approve an asymmetric nature of radical propagation what is also known from Photosystem II $(49,50)$.

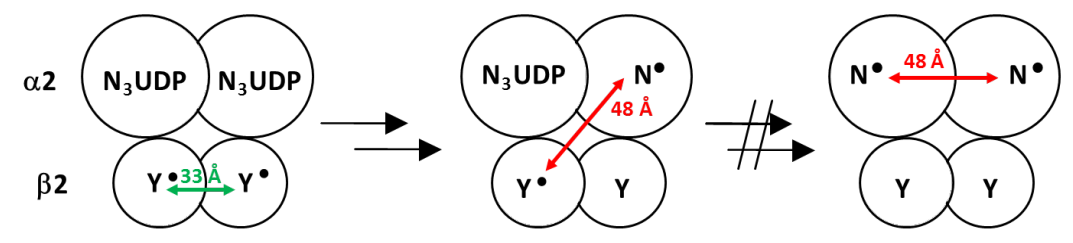

Figure 1-9. Possible models for radical pairs when $\alpha 2 \beta 2$ is inactivated by $N_{3}$ UDP. The left model shows the situation before radical propagation to one of the $\alpha 2$ monomers (before inactivation). The model in the middle and on the right side shows the resulting distances through an asymmetric and symmetric radical propagation. PELDOR and DQC studies could confirm the model in the middle and had to exclude the one on the right side (51).

\subsection{Electron transfer in model proteins}

Before concentrating on proton-coupled electron transfer in RNR a short introduction to electron transfer (ET) reactions in biological systems is necessary in order to understand especially its distance dependency. ET theory was developed by Marcus and Sutin in the 80's and is described by the semiclassical Marcus-Levich relation (Equation 1-1), where $\mathrm{k}_{\mathrm{ET}}$ is the ET rate constant in a reaction between an electron donor (D) and an acceptor (A) held at fixed distance and orientation (52).

$$
k_{E T}(D \rightarrow A)=\frac{4 \pi^{3}}{h^{2} \lambda k_{B} T} H_{D A}^{2} \exp \left\{-\frac{\left(\Delta G^{\circ}+\lambda\right)^{2}}{4 \lambda k_{B} T}\right\}
$$

Equation 1-1. The Marcus-Levich equation describing ET in chemical and biological systems. 
The ET rate depends here on three critical parameters:

1) The driving force for the electron transfer $\Delta \mathbf{G}^{\mathbf{O}}$ which is defined as the reduction potential of the electron acceptor minus the electron donor.

2) The extent of nuclear reorientation $\lambda$ in $D, A$, together with the solvent that accompanies formation of $D^{+}$and $A^{-}$from the equilibrium position to that of the products.

3) And the electronic coupling $\mathbf{H}_{\mathrm{DA}}{ }^{2}$ between the reactants $[D, A]$ and the products $\left[D^{+}, A^{-}\right]$ describing the ET transfer probability at the transition state.

The first two parameters depend on the chemical nature of the redox centers and the solvent nearby the centers, whereas the third is a function of the D-A distance and the structure of the intervening medium (Equation 1-2).

$$
H_{D A}=H_{D A} \cdot \exp ^{-\beta\left(R-R_{0}\right)}
$$

Equation 1-2. Expression for electronic coupling. The probability of electron tunneling is given by $H_{D A}{ }^{2}$. $H_{D A}$ reflects hereby the exponential distance dependence. $R_{0}$ is the center to center distance between the redox partners at van der Waal contact and $\mathrm{R}$ is the center to center distance between the two partners with an intervening medium in between. $\beta$ is a constant and reflects the alacrity at which the exponential wave function decays at fixed D-A distance. $\mathrm{H}_{D A}{ }^{\circ}$ is the electronic coupling at van der Waals contact.

Gray and coworkers have used laser flash-quench methods in Ru-modified proteins to trigger ET reactions and to study medium and distance dependences of long-range electron tunneling between 10 to $30 \AA$ (Figure 1-10) (53). 


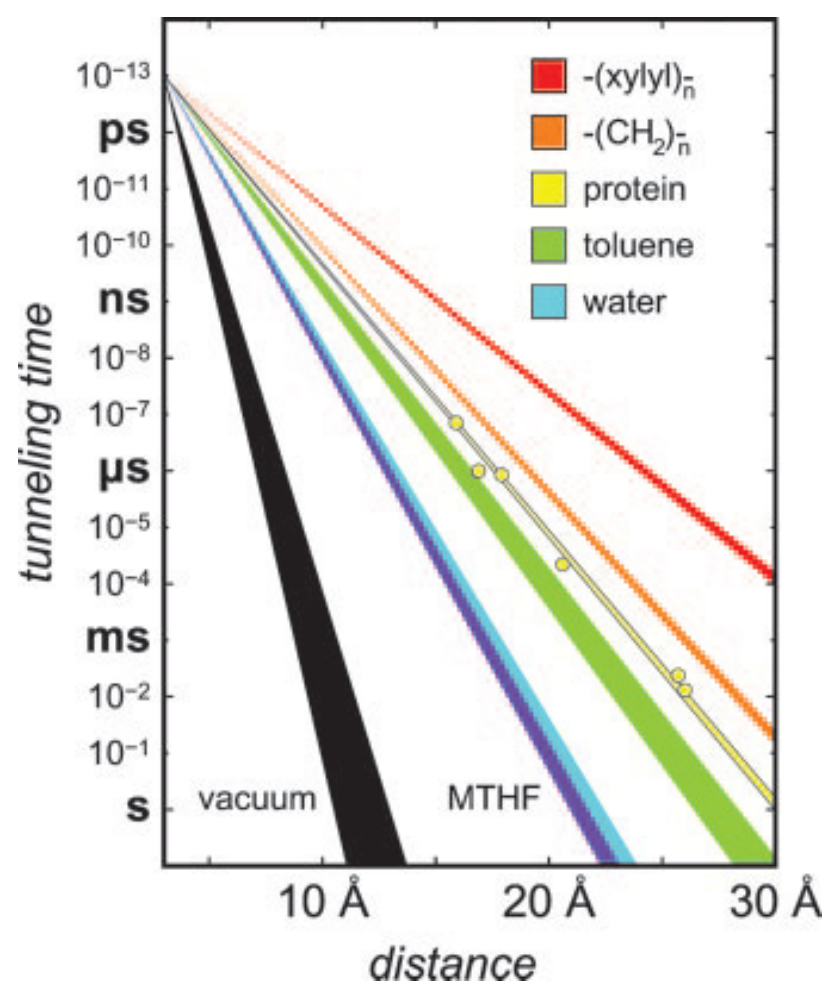

Figure 1-10. Timetable for electron tunneling through various media with respect to the donoracceptor distance. From electron transfer analysis on Ru-modified proteins a direct electron transfer on the second timescale or lower is only possible when the distance between donor and acceptor is less than $30 \AA$. . The turnover rate of E.coli RNR is $2-10 \mathrm{~s}^{-1}$ and the distance gap between $\operatorname{Tyr}_{122}{ }^{\bullet}$ in the $\beta$-subunit and $\mathrm{Cys}_{439}$ in the $\alpha$-subunit is $35 \AA$. The analysis by Gray and Winkler excluded therefore a priori a one-step electron transfer in the RNR enzyme.

From this analysis they could derive for proteins a mean distance decay constant $\beta$ of $1.1 \AA^{-1}$. For comparison a value of $2.9-4 \AA^{-1}$ has been estimated for redox partners separated by vacuum and a value of $1.55-1.65 \AA^{-1}$ for water as an intervening medium. An intervening medium like a protein decreases $\beta$ further and accelerates significantly ET. Increase and decrease in $\beta$ at fixed donor-acceptor distances is exemplified below (Figure 1-11). 

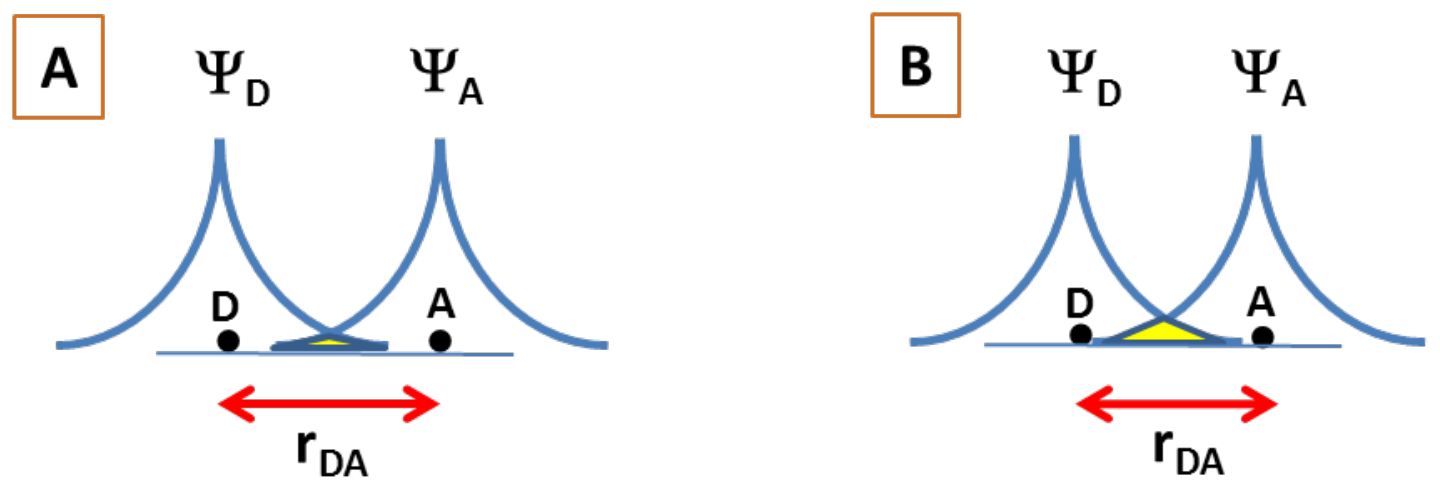

Figure 1-11. Overlapping wave-functions for donor and acceptor at a fixed distance $r_{D A}$. In $A$ ) the wave function overlap (see yellow area) is small due to large $\beta$. In $B$ ) the wave function overlap increases due to smaller $\beta$. $\beta$ is therefore a measure of the height of the tunneling barrier.

The importance of intervening aromatic amino acid residues in ET-proteins has been also recognized and showed the reduction of the $\beta$-value and increase of electron coupling. Especially when the ET distance gets longer (> $25 \AA$ ) electron hopping over redox-acitve amino acids is necessary in order to account for ET rates which can justify a reasonable activity in redox-active enzymes.

Calculations on Ru-modified azurins with an intervening Tyr showed that the hopping mechanism for the redox-triad $\mathrm{Cu}^{+} \rightarrow \mathrm{Tyr}^{+/ 0} \rightarrow \mathrm{Ru}^{3+}$ is more favorable than the direct ETtransfer for the redox-couple $\mathrm{Cu}^{+} \rightarrow \mathrm{Ru}^{3+}(54)$ and experimental studies by Giese (55) with model peptides which allow multistep hopping processes to be analyzed showed that an intervening amino acid Cys accelerates the ET reaction about 30 times. Cys was placed hereby right in the middle of the peptide (length of $20 \AA$ ). Moreover a kinetic isotope effect of 2 clearly demonstrated that water plays a significant role in ET across the peptide.

Accurate ET analysis in model proteins and polymers paved the way for dissecting ET reactions that occur in photosynthetic and respiratory enzymes involving metal cofactors (56). However detailed analysis of proton transfer reactions accompanying ET reactions in biological systems is still a very new research area.

E.coli RNR exhibits exactly these amino acids on its radical propagation pathway which were mentioned above together with Trp and the insights from the ET studies like for example the dependence of the ET rates on the D-A distance were of importance for the analysis of a multistep ET over $35 \AA$. 


\subsection{Proton-coupled electron transfer (PCET) in RNR}

The term proton-coupled electron transfer (PCET) was introduced by Huynh and Meyer in 1981. PCET is intrinsically a quantum mechanical effect because both the electron and proton tunnel, owing to overlap between the donor and acceptor wavefunctions. E.coli RNR serves as a natural system in which PCET events are now studied at each stage of charge transfer. A model for several reversible PCET events in RNR is shown below (Figure 1-12).

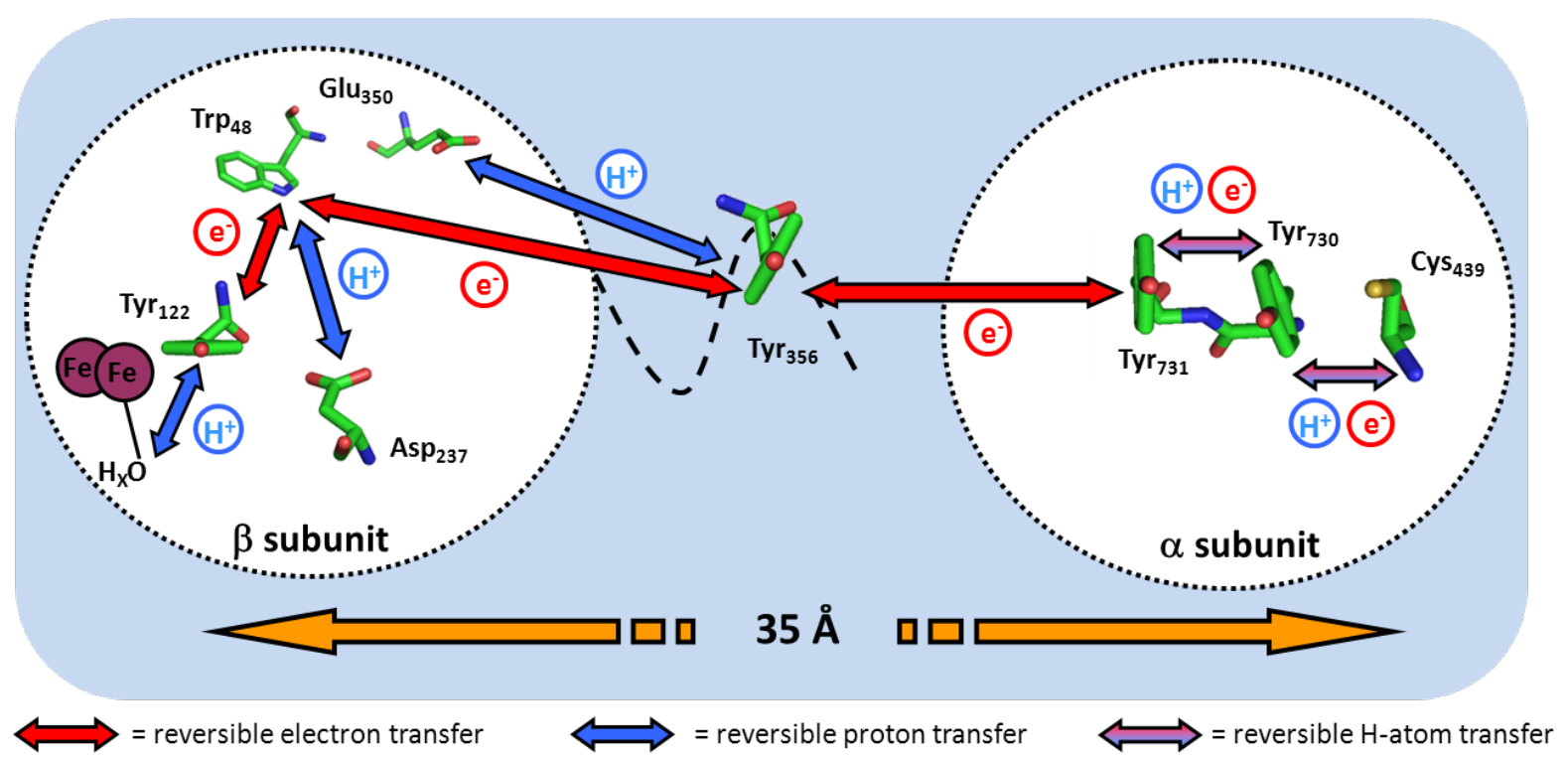

Figure 1-12. The RNR PCET-model showing the long-range charge transfer over $35 \AA$ starting at $Y_{122}$ in the $\beta$-subunit and reaching $C_{439}$ in the $\alpha$-subunit. Three different transfer events are indicated with electron and proton either migrating separately or in parallel. The model consists of the conserved amino acid residues which were shown in Figure 1-6.

As the enzyme shows the highest activity at nearly neutral $\mathrm{pH}(\sim 7.6)$ a one-electron oxidation of amino-acid residues like Tyr, Trp and Cys requires loss of both, an electron and a proton. Without a coupled movement of proton and electron the oxidation of tyrosine residues in RNR is hardly possible.

If the electron would have to be transferred in the absence of proton transfer first a very high oxidizing potential is needed ( $E=1.46 \mathrm{~V}$ vs $\mathrm{NHE}$ ) and second the very strong acid $\mathrm{TyrOH}^{\bullet+}\left(\mathrm{pK}_{\mathrm{a}}=-2\right)$ is produced. Both scenarios are highly unlikely in biological systems. The 
high-energy pathways can be avoided if electron and proton transfers are coupled to each other and finally generate a Tyr ${ }^{\bullet}$.

Besides for the stable $\operatorname{Tyr}_{122}{ }^{\bullet}$ which has a $t_{1 / 2}$ of four days direct evidence for the other three $\operatorname{Tyr}^{\bullet}\left(\operatorname{Tyr}_{356}{ }^{\circ}, \operatorname{Tyr}_{731^{\circ}}\right.$ and $\left.\operatorname{Tyr}_{730^{\circ}}\right)$ and the generation of the Cys ${ }^{\circ}$ and $\operatorname{Trp}^{\bullet}$ has been missing so far during nucleotide turnover in RNR.

In the early 90's a formalism for the PCET reaction began to appear with the need to account for both, electron and proton movement (57). This was enabled by the development of hydrogen-bonded donor-acceptor complexes with $\mathrm{Zn}(\mathrm{II})$ porphyrins or $\mathrm{Ru}(\mathrm{II})$ polypyridyl serving as donors and organic aromatic compounds serving as acceptors (58) (Figure 1-13).

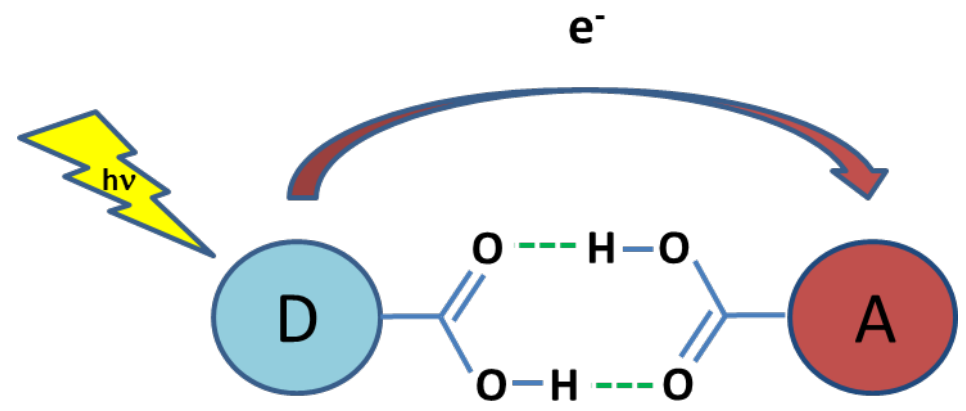

Figure 1-13. PCET model system for a symmetric dicarboxylate hydrogen-bond interface. The donor (D) transfers hereby an electron after photoexcitation $(580 \mathrm{~nm})$ to the acceptor (A). In one of the first model systems $D$ is a $Z n(I I)$ porphyrin and $A$ is 3,4-dinitrobenzoic acid.

From these model systems it could be theoretically derived and also experimentally demonstrated that isotopic substitution of protons through deuterons has a pronounced effect on the electron transfer rate $\left(k_{E T}(H) / k_{E T}(D)=1.6\right.$ for forward $E T$ and $k_{E T}(H) / k_{E T}(D)=1.7$ for reverse ET).

On the basis of these results which demonstrated that proton movement is accompanied by ET in hydrogen-bonded complexes two different transfer situations for proton and electron movement are proposed. The different pathways can be described within a parallelogram (Figure 1-14) (59). 

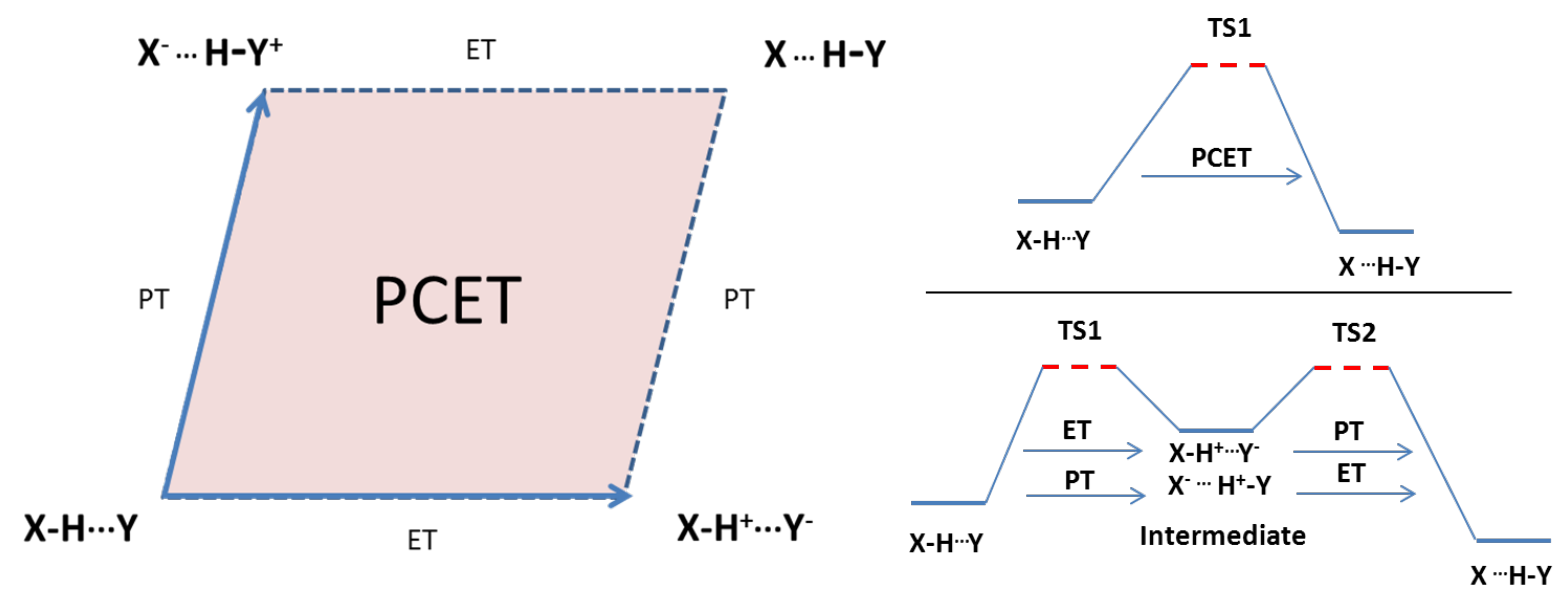

Figure 1-14. (Left) Scheme for proton-coupled electron transfer. $X$ is the electron transfer (ET) and proton transfer (PT) donor and $\mathrm{Y}$ is the acceptor. The edges of the parallelogram describe step wise PT/ET or ET/PT. The diagonal path and the area of the parallelogram describe the PCET process. (Right up) Kinetic diagram for PCET consisting of one transition state (TS1) and for step wise PT/ET or ET/PT (Right bottom) consisting of two transition states and one Zwitterion intermediate.

Two scenarios are described by this representation. Proton and electron can move in a stepwise fashion along the edges or they move coupled to each other including the entire space within the parallelogram. In the stepwise mechanism an intermediate is formed (either $\mathrm{X}-\mathrm{H}^{+\cdots} \mathrm{Y}^{-}$or $\mathrm{X}^{-\cdots} \mathrm{H}^{+}-\mathrm{Y}^{+}$) and in the PCET mechanism a single transition state is formed with no intermediate states populated along the reaction coordinate.

PCET along the diagonal of the parallelogram accounts for $\mathrm{H}$-atom transfer - a subset of PCET - with minimal charge buildup in the transition state. In order to manipulate PCET transition states through thermodynamic parameters one can change $\mathrm{pK}_{a} \mathrm{~s}$ or reduction potentials which drive the charge to separate to a more PT-like transition state or ET-like transition state. In the pure stepwise mechanism one can treat the kinetics as separate ET and PT kinetics. For this mechanism a proton transfer rate is combined with an electron transfer rate in a simple formula (Equation 1-3).

$$
k_{E T / P T}=\frac{k_{E T} k_{P T}}{k_{E T}+k_{P T}}
$$

Equation 1-3. Kinetic expression for consecutive electron and proton transfer. 
On the other hand, for the PCET event the kinetics are more complicated. Savéant and coworkers could simplify a formalism by Hammes-Schiffer to study PCET oxidation of hydrogen-bonded aminophenols in which the phenolic proton is transferred to the nitrogen atom in a concerted way with the electron (Figure 1-15) (Equation 1-4) (60).

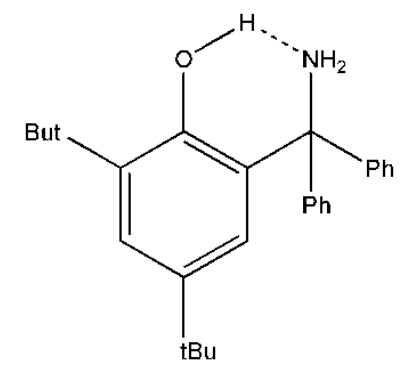

Figure 1-15. Hydrogen-bonded aminophenol that undergoes a concerted proton-electron transfer reaction.

$$
k_{P C E T}=Z \exp \left[\frac{-\lambda}{4 R T}\left(1+\frac{\Delta G^{\circ}}{\lambda}\right)^{2}-\frac{\Delta Z P E}{R T}\right]
$$

Equation 1-4. Kinetic expression for concerted proton-coupled electron transfer in hydrogen-bonded aminophenols. $Z$ is related hereby to the probability of proton tunneling and ET. $\triangle$ ZPE is the change in zero-point energy of the proton vibration at the transition state as compared to the reactant state which can be estimated through the deuterium isotope effect for an adiabatic reaction (proton and deuterium transfer occurring on the electronic ground state). $\Delta \mathrm{G}^{\circ}$ and $\lambda$ have been explained before.

The proton rest mass is $\sim 2000$ times that of the electron and through the de Broglie relation $(\lambda=h / p)$ one can calculate a proton wavelength which is $\sim 40$ times shorter than that of an electron at a fixed energy. As a consequence, PT is limited to short distances, whereas the electron, as the lighter particle, may transfer over very long distances. In enzymes different transfer distances are possible. When electron and proton are transferred together, via hydrogen atom transfer, transfer distances are kept short within hydrogen-bond contact. For the purposes of long-distance transport proton and electron take two different ways and the movement is said to be orthogonalized by the enzyme (Figure 1-16). 
A

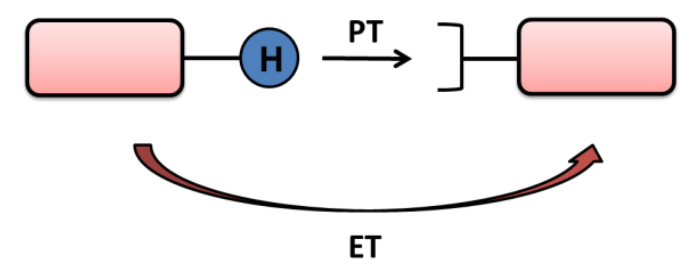

B

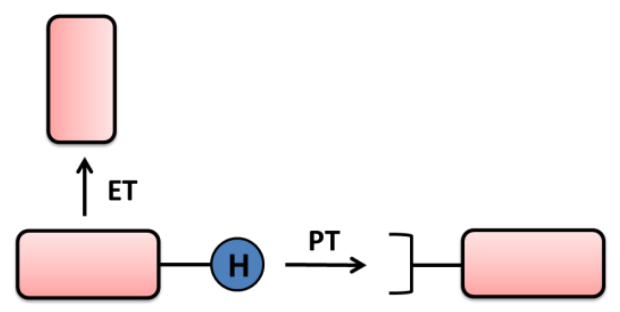

Figure 1-16. Scheme for two different PCET scenarios occurring in enzymes. A) In the unidirectional PCET proton and electron move into the same direction. B) In the bidirectional PCET electron and proton move into two different directions.

PCET analysis in enzymes have been performed so far on E.coli RNR and photosystem II (61) . A model that accounts for PCET in RNR is shown above (see Figure 1-12). In this model in the $\beta$-subunit only bidirectional PCET's are proposed and in the $\alpha$-subunit unidirectional PCET's due to short distances between $Y_{731}, Y_{730}$ and $C_{439}$ (3 - $4 \AA$ distance range).

In order to examine the PCET unnatural amino acids have been incorporated on the PCET pathway through site-specific replacement of the three tyrosines $Y_{356}, Y_{731}$ and $Y_{730}$. Using intein protein ligation methods, semisynthetic $\beta 2$ subunits were generated with unnatural amino acids at position $356(62,63)$. Also a series of fluorinated tyrosine derivatives have been generated with reduction potentials that varied from -50 to $+270 \mathrm{mV}$ relative to tyrosine and with $\mathrm{pK}_{\mathrm{a}} \mathrm{s}$ that ranged from 5.6 to 9.9 (64). The $\mathrm{pH}$ rate profiles of deoxynucleotide production for semisynthetic $\mathrm{F}_{n} \mathrm{Y}_{356}-\beta 2 \mathrm{~s}$ were reported and the results suggested that the rate-determining step for RNR activity could be changed from a physical (conformational) step to the radical propagation step through alteration of reduction potentials at one amino acid position, $\beta-356$ (65). Another insight from these studies was that although several of these analogous were deprotonated at $\mathrm{pH}>7.5$ nucleotide reduction could take place. The conclusion was that a proton is not obligated to propagate together with the electron on the pathway. Therefore an orthogonal PCET is suggested on position 356 where electron movement is directed towards $W_{48}$ and $Y_{731}$ and a proton movement to the conserved $E_{350}$. A spectroscopic evidence for an orthogonal PCET is however missing.

The incorporation of radical spin traps which should function as reporters for hydrogen-bonding networks at position 356, 730 and 731 began 2006 . 3,4dihydroxyphenylalanine (DOPA) was incorporated with the semisynthetic intein method (66) 
whereas 3-aminotyrosine $\left(\mathrm{NH}_{2} \mathrm{Y}\right)$ has been used to exchange $Y_{730}$ and $Y_{731}$ respectively through the suppressor tRNA/aminoacyl-tRNA synthetase methodology (67). At all three positions the propagating radical on one $\alpha \beta$ side was trapped due to the lower redoxpotential of DOPA and $\mathrm{NH}_{2} \mathrm{Y}$. DOPA's redox-potential is $260 \mathrm{mV}$ lower at $\mathrm{pH} 7.0$ in comparison to Tyr whereas $\mathrm{NH}_{2} \mathrm{Y}^{\prime}$ s redox-potential is $190 \mathrm{mV}$ lower at the same pH. First electron-electron distance measurements between the $\mathrm{Y}_{122}{ }^{\bullet}$ in the $\beta$-subunit and $\beta$ $\mathrm{DOPA}_{356^{\circ}}, \alpha-\mathrm{NH}_{2} \mathrm{Y}_{731}{ }^{\circ}$ and $\alpha-\mathrm{NH}_{2} \mathrm{Y}_{730^{\circ}}$ supported the asymmetric radical transfer and the $\alpha 2 \beta 2$ docking model (68). For the first time the distance to residue 356 provided the first structural constraint, which is not visible in all structures of $\beta 2$ solved to date $\left(\beta-Y_{122}{ }^{\bullet}-\beta\right.$ $\mathrm{Y}_{356}{ }^{\bullet}$ distance $=31 \AA$ A). Additionally to that we performed high-field PELDOR studies between $\beta-Y_{122}{ }^{\circ}$ and $\alpha-N_{2} Y_{731}$ in order to clarify the relative orientation between the two radicals (69). Performing these studies additionally with the $\beta-Y_{122} \cdot \beta-Y_{356} \cdot$ pair would allow one to determine the unknown distance between $\beta-Y_{356}$ and $\alpha-Y_{731}$.

In the next three sections the Schultz method for incorporation of the radical spin trap $\mathrm{NH}_{2} \mathrm{Y}$ at position 730 and 731 in the $\alpha$-subunit, the high-frequency/multifrequency EPR technique which is used for detailed examination of the $\mathrm{NH}_{2} \mathrm{Y}^{\bullet}$ and the high-field ENDOR method for the detection of hydrogen-bonding networks during PCET around $\mathrm{NH}_{2} \mathrm{Y}^{\circ} \mathrm{S}$ in the $\alpha$ subunit are discussed.

\subsection{Suppressor tRNA/aminoacyl-tRNA synthetase methodology}

A robust in vivo method for site-specific incorporation of unnatural amino acids has been evolved by Schultz and his coworkers Chin and Mehl. This method uses a selected aminoacyl-tRNA synthetase (RS) from a library of other synthetases that is specific for the unnatural amino acid of interest (here $\mathrm{NH}_{2} \mathrm{Y}$ ) and which recognizes a cognate tRNA selected also from a library of amber suppressor tRNA ${ }^{\mathrm{Tyr}}$ s containing the stop anticodon AUC (70) (Figure 1-17). 


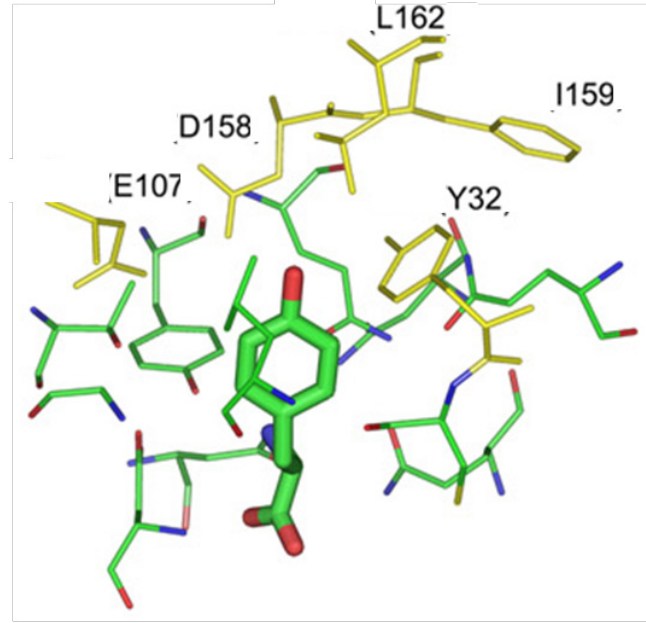

M.jannaschii TyrRS mutants

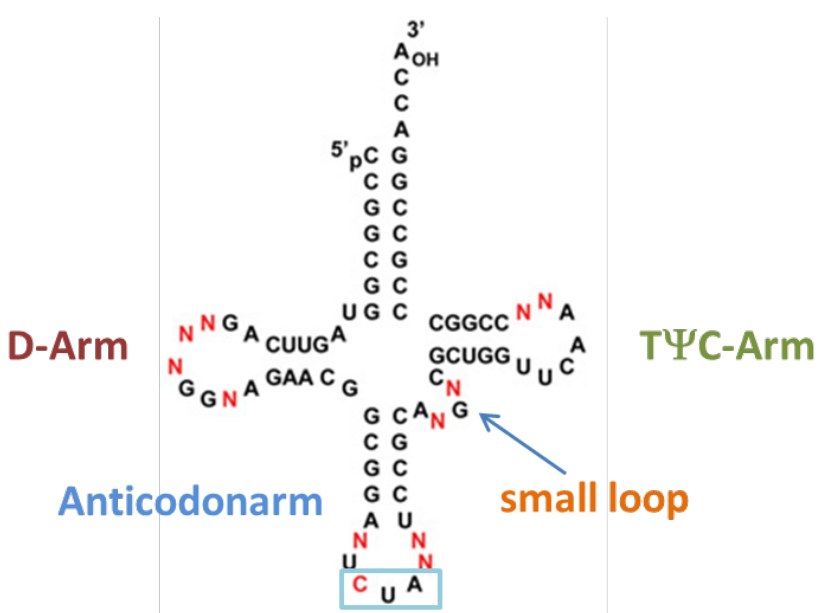

Amber suppressor tRNA ${ }^{\mathrm{T} Y r_{S}}$

Figure 1-17. Library of Methanococcus jannaschii TyrRS mutants, which was generated by randomizing five residues shown in yellow (Tyr32, Glu107, Asp158, lle159 and Leu162) making it highly specific to the unnatural amino acid (UAA) of choice (left). Additionally a library was generated of amber suppressor tRNAs which is based on M.jannaschii tRNA ${ }^{\text {Tyr }}$ where 11 nucleotides were randomized in different regions of the tRNA (mutRNA) (right).

Importantly the tRNA/RS pair is orthogonal to the natural occurring tRNA/RS pairs in the host (here E.coli). This means that the amber suppressor tRNA cannot cross-react with any in vivo RSs, and the evolved RS cannot charge any in vivo tRNAs with a natural or unnatural amino acid (UAA). In addition, the in vivo RSs are not able to charge their cognate tRNAs with the unnatural amino acid. Finally, the unnatural amino acid must be taken up by the cell and must not be toxic. All the criteria mentioned above have to be met in order to express the protein of interest with the unnatural amino acid incorporated with high fidelity at a specific location.

Until 2010 nearly 70 unnatural amino acids have been incorporated with two being hydroxy acids (71). One class of theses UAAs contains chemically reactive groups that can be sitespecifically modified in vitro and in some cases in vivo with selective chemistries including oxime condensation reactions, click chemistry, Michael addition reactions and Suzuki couplings. Another class of UAAs contains in vitro or cellular probes of protein structure and function. They can be used as IR, NMR, and fluorescent probes, as well as redox-active reagents (EPR probes) $(67,72)$, heavy atom probes for X-ray structure determination and 
probes of hydrogen-bonding and packing interactions in proteins. Some UAAs have photocrosslinking, metal ion-binding or photoreactive abilities whereas others correspond to the product of a posttranslational modification.

Most of the UAA's are encoded in the E.coli organism following by the yeast and mammalian host as expression system. In E.coli the $M$. jannaschii tRNA/RS pair has been manipulated to fit the criteria for orthogonality. In yeast and mammalian cells, the E.coli tRNA/RS pair has been used so far. The strategy to import a heterologous tRNA/RS pair from a different lifedomain or species into another one has been shown to be the best solution for creating orthogonal tRNA/RS pairs where mostly amber nonsense codons (UAG) have been used as stop codons for the recognition of the evolved tRNA. To a lesser extent, the ochre, opal or frameshift codons could be used to specify UAA's. As an example the incorporation of the unnatural amino acid $\mathrm{NH}_{2} \mathrm{Y}$ into the $\alpha$-subunit of E.coli is schematically shown, using hereby as a blank codon the amber suppressor codon (Figure 1-18). For this purpose the $\mathrm{NH}_{2} \mathrm{Y}$-specif M. jannaschii aminoacyl-tRNA synthetase $\left(\mathrm{NH}_{2} \mathrm{Y}-\mathrm{RS}\right)$ and the appropriate $M$. jannaschii amber suppressor tRNA have been evolved in the laboratory of Peter Schultz in order to incorporate $\mathrm{NH}_{2} \mathrm{Y}$ at residue positions 731 and 730 in the $\alpha$-subunit (67). Only recently the incorporation of $\mathrm{NH}_{2} \mathrm{Y}$ at position 356 in the $\beta$-subunit was successful (73). It has to be mentioned that although DOPA could be incorporated into proteins like myoglobin (Mb) $\mathrm{NH}_{2} \mathrm{Y}$ was found to be more stable to oxidation than DOPA therefore making it a more practical target. 

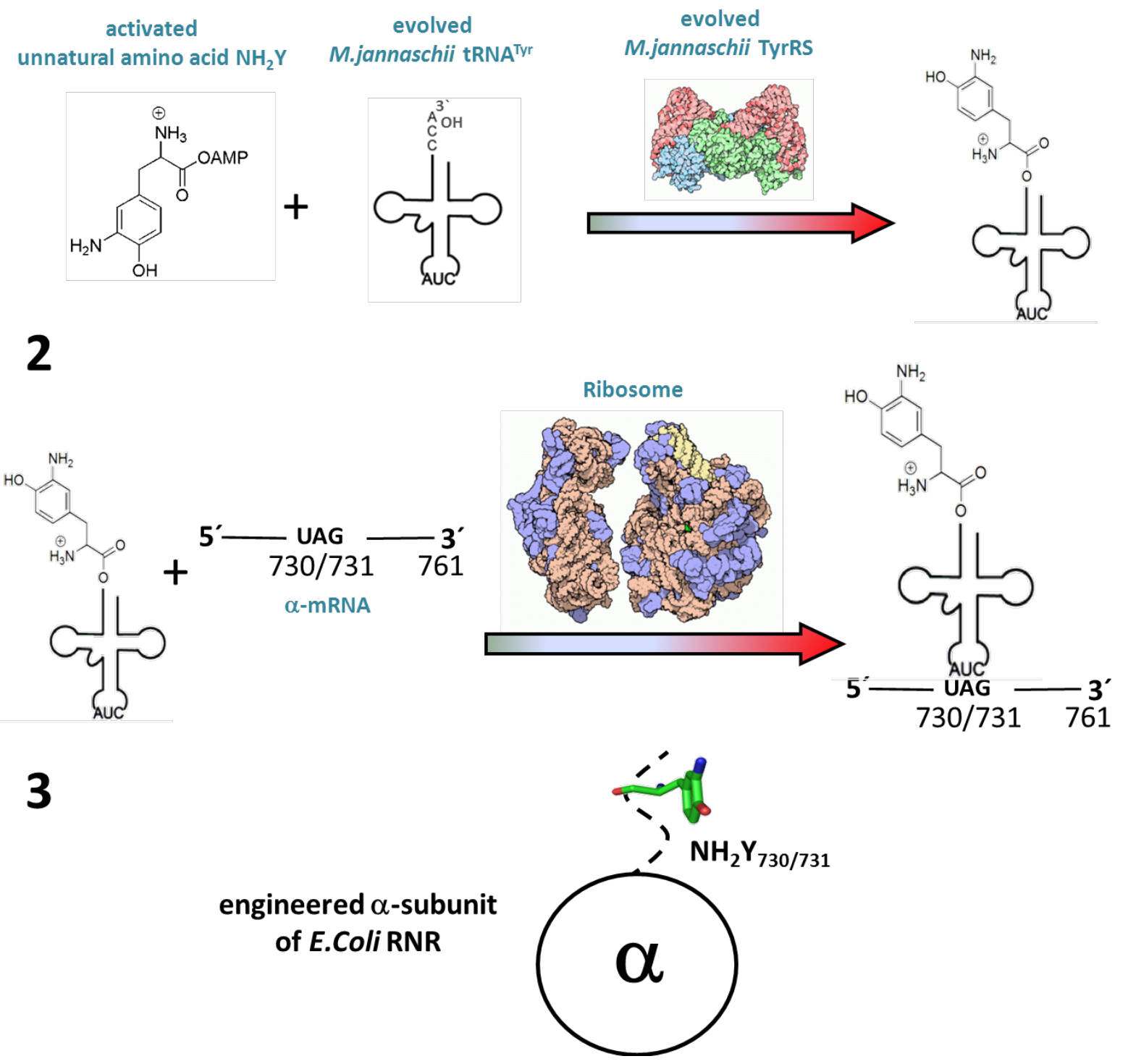

Figure 1-18. In vivo incorporation of $\mathrm{NH}_{2} \mathrm{Y}$ into the $\alpha$-subunit. In the upper scheme (1) the evolved tRNA synthetase covalently attaches the activated $\mathrm{NH}_{2} \mathrm{Y}$ to the $3^{-}-\mathrm{OH}$ of the evolved tRNA. In the scheme in the middle (2) a ribosome (left: the $30 \mathrm{~S}$ small subunit, right: the $50 \mathrm{~S}$ large subunit) performs the translation process. The amber stop codon on the $\alpha$-mRNA codes hereby for the unnatural amino acid. The last scheme (3) shows the incorporated $\mathrm{NH}_{2} \mathrm{Y}$ into the $\alpha$-subunit either on position 730 or position 731 .

The generation of new biological, chemical and physical properties in proteins is now widely applicable through systematic genetic encoding of new UAAs. Furthermore the combination of multiple blank codons with multiple orthogonal UAA tRNA/RS pairs creates proteins with different structural and functional properties. A completely new approach of multiple UAAs $\mathbf{4 0} \mid \mathrm{P}$ a g e 
incorporation into proteins can be performed by using an orthogonal ribosome that efficiently decodes a series of quadruplet codons together with the amber stop codon. Until now the orthogonal ribosome has been combined with two orthogonal tRNA/RS pairs with the result to incorporate an alkyne and an azide as unnatural amino acids with subsequent performance of click chemistry (74). As the orthogonal ribosome, called ribo-Q1, efficiently decodes several blank codons on an orthogonal mRNA, one could use this approach in order to create peptides or proteins which exhibit solely UAAs.

\subsection{High-frequency EPR spectroscopy and the usage of the multifrequency approach}

High-frequency/multifrequency EPR spectroscopy and its application for the investigation of structure and dynamics of paramagnetic compounds is a quite new and prominent field in EPR spectroscopy since the establishment of high-field spectrometers (> 3 T) (75).

Measurements at high-frequencies in combination with a double resonance technique such as ENDOR (see section below) or PELDOR find today wide application and provide structural information of biological systems originating from magnetic interactions between nuclei and the center of the free radical and through long-range magnetic interactions between two unpaired spins (Figure 1-19) (76). 


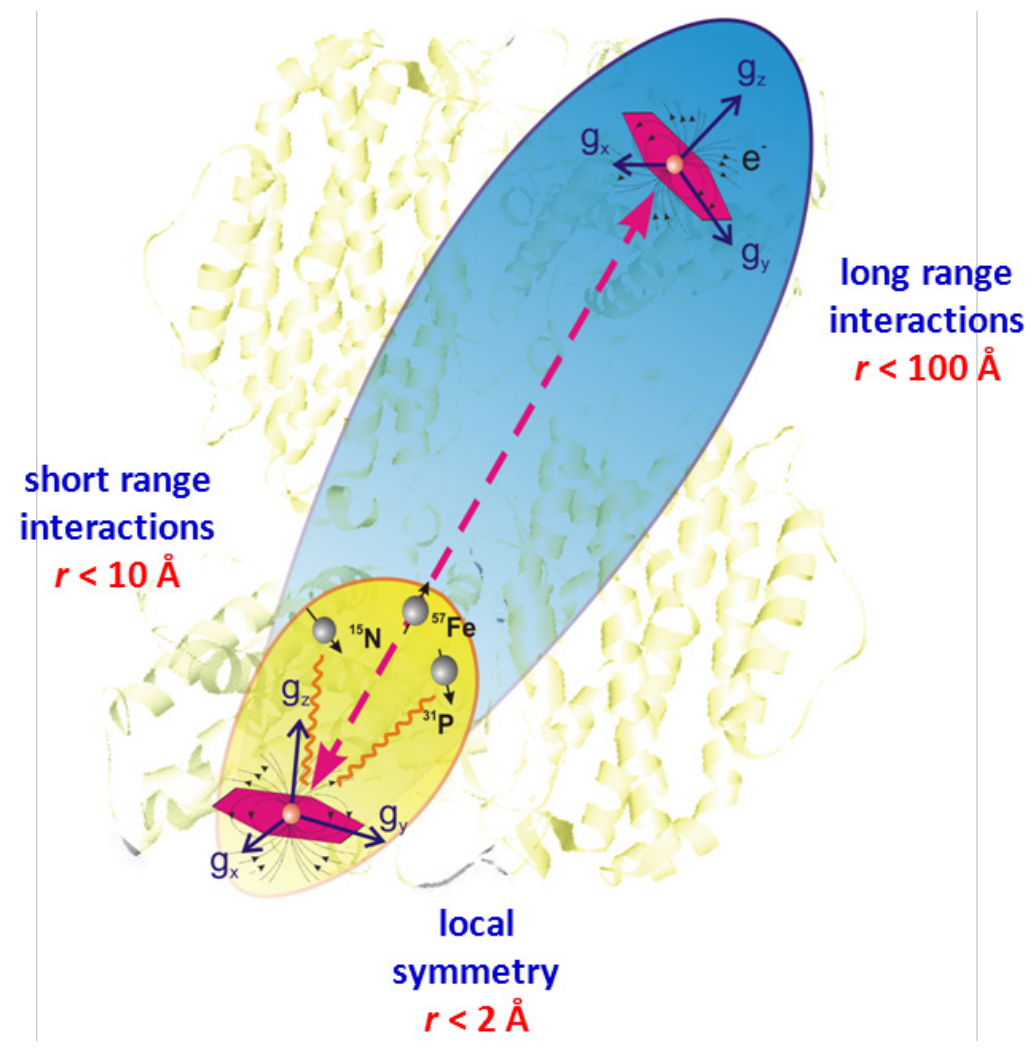

Figure 1-19. Scheme for various magnetic interactions between radicals and their surrounding environment in a biomolecule that can be detected with high-frequency EPR spectroscopy. Long range interactions can be monitored through PELDOR an electron-electron double resonance technique which determines distances $(r<100 \AA)$ and orientations between two radicals. The hyperfine interaction between electrons and nuclei occur in a smaller radius and depend further on the kind of nucleus. They can be monitored through ENDOR an electron nuclear double resonance technique. For ${ }^{1} \mathrm{H}^{\prime} \mathrm{s}$ the interaction range can be up to $10 \AA$ for ${ }^{2} \mathrm{H}^{\prime} \mathrm{s}$ it is up to $4.5 \AA$. . Changes in the local symmetry $(r<2 \AA)$ and the hydrogen-bond environment can further be monitored through changes in the g-values.

At high frequencies ( $\geq 95 \mathrm{GHz}$ ) the EPR spectra are sufficiently spread out in the field dimension and one can extract the g-values of organic radicals in a very precise way (Figure 1-20). The higher the frequency the more accurate is hereby the g-value or g-tensor analysis. Slight shifts of the g-values (corresponds to the chemical shift in NMR) are easily detectable at high frequencies and are used to monitor the local nucleic surrounding of a radical. The strong g-anisotropy at high frequencies allows also the application of so-called orientation selective experiments. The term anisotropy (expressed as a tensor) considers hereby an orientation dependence of a physical property (here g-value) which can be 
visualized in 3D-spheres (see Figure 1-18 at the bottom) but is generally used for all magnetic properties in EPR spectroscopy like i.e. the dipole-dipole interaction.

These experiments allow in combination with a double resonance technique such as ENDOR, to get out the mutual orientation of the $g$ and hyperfine tensor. Hereby one excites with different pulse strengths only certain regions in the EPR spectrum (i.e. at B $\| g_{x x}$ or at B $\left.\| g_{y y}\right)$ while performing the double resonance experiment.
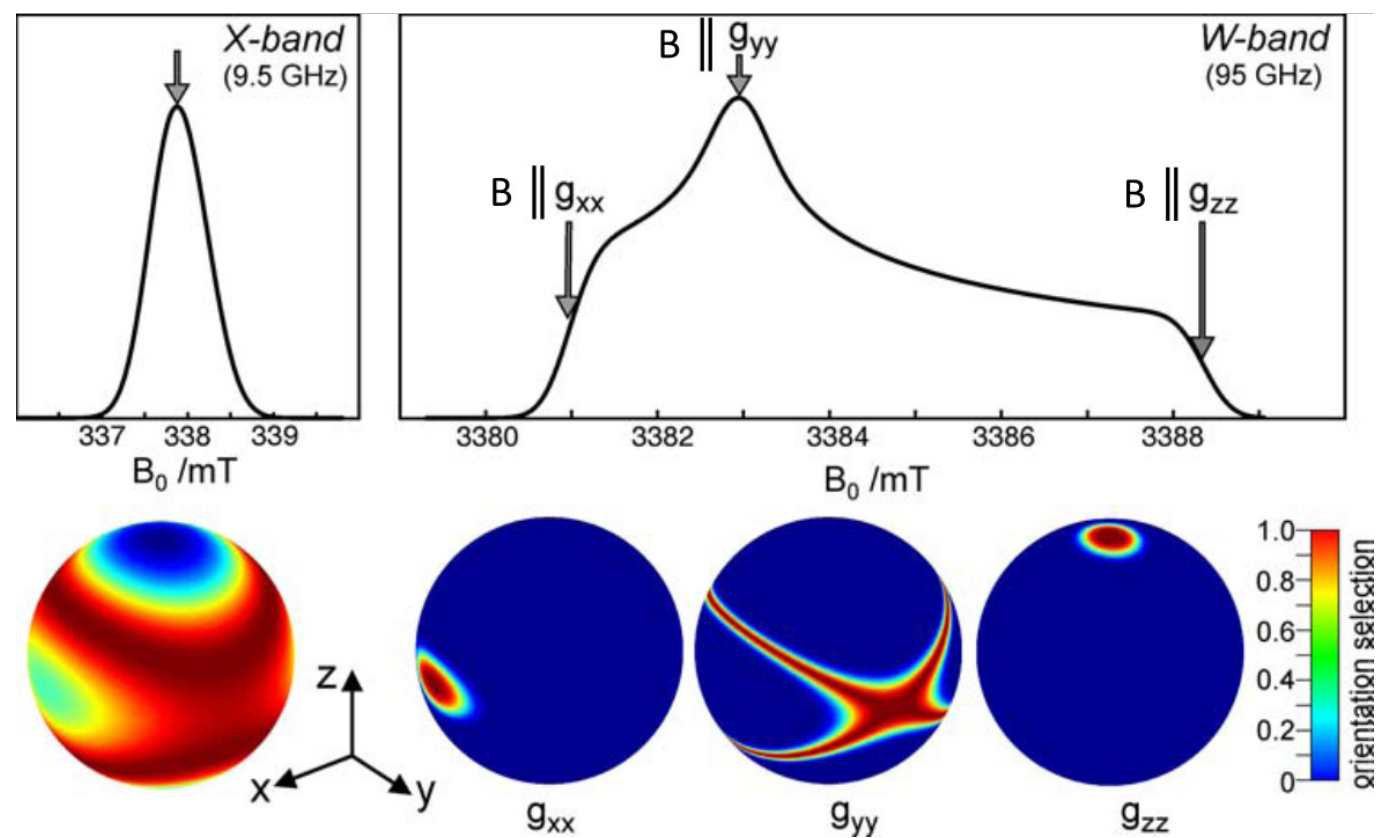

Figure 1-20. Orientation selection by high-field EPR exemplified on the anion radical of the ubiquinone acceptor cofactor in frozen-solution bacterial photosynthetic RCs. Left: X-band spectrum with no g-resolution. Right: W-band spectrum with enhanced g-resolution which allows for orientation selective experiments at $95 \mathrm{GHz}$.

The zero-field splitting (ZFS) which describes various interactions of the energy levels in the absence of an applied magnetic field for an electron spin with $S>1 / 2$, can lead to very broad lines in powders or frozen solutions at X-band frequencies. However, as the Zeeman interaction (interaction of an electron with the magnetic field) is more dominant at higher frequencies than the ZFS the EPR spectral shapes become simpler to analyze at high frequencies. Therefore going to higher frequencies lead in general also to more accurate determination of the g-factors for transition metal ions. 
In going to higher frequencies allows also the study of fast motions of EPR probes (picosecond to nanosecond). For example rotational motions which are in this time regime can be visualized for spin labels attached to a macromolecule when going to high frequencies rather than at X-band frequency (77). A descriptive example can be found in the calculation of EPR spectra measured at different frequencies (multifrequency approach at 9, 94 and $170 \mathrm{GHz}$ ) for spin labeled T4 lysozyme allowing an extraction of an effective rotational correlation time $\left(\tau_{R}\right)$ of the MTSL spin label at two different solvent-exposed sites on the surface of $\alpha$-helices (78).

Another benefit when performing EPR at high frequencies is the better signal-to-noise ratio in comparison to X-band if the sample is limited in size and is not lossy (see Table 1-1 for polarization). Otherwise one can go to lower frequencies. This is an important aspect when working with biological samples where radical concentrations can be $30-50 \mu \mathrm{M}$ or even lower.

\begin{tabular}{|c|c|c|c|c|}
\hline $\begin{array}{c}\text { Frequency } \\
\text { band }\end{array}$ & $\begin{array}{c}\text { MW frequencies } \\
(\mathrm{GHz})\end{array}$ & $\begin{array}{c}\text { Magnetic } \\
\text { field (T) }\end{array}$ & $\begin{array}{c}\text { Sample tube OD } \\
(\mathbf{m m})\end{array}$ & Polarization \\
\hline \hline $\mathrm{L}$ & $\sim 1(1-2)$ & 0.035 & 30 & $7.9 \times 10^{-5}$ \\
\hline $\mathrm{X}$ & $\sim 9.8(8.2-12.4)$ & 0.350 & 4 & $7.9 \times 10^{-4}$ \\
\hline $\mathrm{Q}$ & $\sim 34(26.5-40)$ & 1.2 & 2 & $3.2 \times 10^{-3}$ \\
\hline W & $\sim 94(75-110)$ & 3.35 & 0.9 & $7.9 \times 10^{-3}$ \\
\hline D & $\sim 140(110-170)$ & 5 & 0.5 & $1.1 \times 10^{-2}$ \\
\hline Far infra-red & $\begin{array}{c}170,270,360 \\
(140-1120)\end{array}$ & $\begin{array}{c}9.8 \mathrm{for} \\
270 \mathrm{GHz}\end{array}$ & 0.1 & $2.2 \times 10^{-2}$ \\
\hline
\end{tabular}

Table 1-1: Commonly used frequency bands in EPR spectrometers. The wavelength, the magnetic field, the outer diameter (OD) of the used sample tubes together with the polarization $\Delta \mathrm{n} / \mathrm{n}$ at $298 \mathrm{~K}$ which reflects the sensitivity for different types of spectrometers are given for frequencies up to 270 GHz. For far infra-red measurements Fébry-Perot resonators are used which have an open structure and what makes them accessible for optical excitation. For all other frequency bands closed resonators are used which have either a rectangular or cylindrical shape. 
A last advantage when going to high frequencies will be exemplified in the ENDOR section which concerns the increased nuclear Zeeman resolution leading to separation of ENDOR signals from different nuclei which would significantly overlap at X-band frequency.

There are also disadvantages in high-field EPR to mention:

1) Measurements at higher frequencies of samples with high dielectric losses (lossy samples), such as pure aqueous samples are less convenient because of problems related to the absorption and penetration of microwaves.

2) The samples are very thin and it is often difficult to control oxygen concentrations, ice formations outside the tube or an adequate packing of the sample.

3) One more difficulty when going to higher frequencies is that the EPR linewidth increases at higher frequencies due to $A^{-}, \mathrm{g}$ - and D-strain broadenings, which can mask small hyperfine splittings, resulting from the coupling of nuclei to electrons.

Without any doubt the most commonly used frequency for EPR is still X-band ( $9.5 \mathrm{GHz}$ ), requiring a wave-guide resonator of the size of $\sim 3.0 \mathrm{~cm}$. Despite all of the conveniences offered by $X$-band, like the availability of highly engineered microwave components, the high homogenity of electromagnetic fields through the usage of iron-core electromagnets as well as the easier handling of EPR samples (EPR tubes are of the size of NMR tubes), EPR at high frequencies becomes more and more an attractive field for the characterization of new organic radicals and their environment in biological systems. In many countries national laboratories have been funded (i.e. US, France, Japan) which make high-field EPR facilities available to many research groups. Furthermore home-built apparatus are implemented in many labs around the world with the first pulsed high-field EPR spectrometer built 1989 in Leiden at $95 \mathrm{GHz}$ (79), followed by two spectrometers operating at $140 \mathrm{GHz}$ from Moskau and Cambridge/USA $(80,81)$ and a $180 \mathrm{GHz}$ spectrometer built in Frankfurt am Main (82). Since 1996 a 95-GHz pulsed high-field EPR spectrometer is commercially available from Bruker Analytik and is also implemented in our high-field EPR lab at the MPIBPC since 2008. In this Thesis measurements on the $\mathrm{NH}_{2} \mathrm{Y}^{\bullet}$ were performed mostly with this spectrometer. 
In order to demonstrate the impact of high-frequency EPR spectroscopy three studies which are shown below have been chosen as descriptive examples.

The first study deals with the characterization of spin adducts of carbon centered radicals which have nearly the same $g$ factors. Measurements of a mixture of the two organic radicals, phenyl-PBN and trichloromethyl-PBN (PBN = phenyl tert-butylnitrone) could be easier resolved at $\mathrm{W}$-band frequency. The stronger electron Zeeman interaction, which is proportional to the applied magnetic field, is hereby of advantage to disentangle radical species with very small difference in g-values. At X-band the EPR spectrum of the PBN radical mixture shows strong overlap, however at W-band one is able to discriminate between phenyl-PBN and trichloromethyl-PBN radicals (Figure 1-21) (83).

Mixture of phenyl-PBN and
trichloromethyl-PBN at X-band

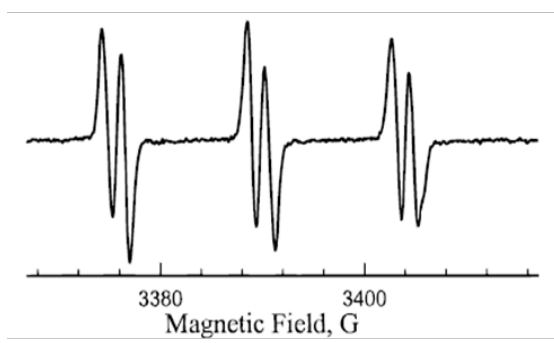

Mixture of phenyl-PBN and
trichloromethyl-PBN at W-band

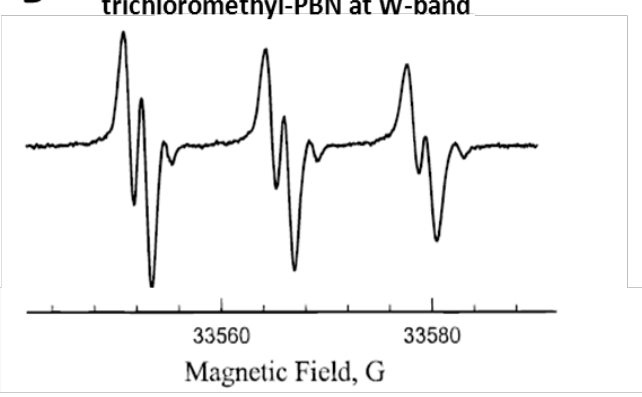

Simulation of phenyl-PBN

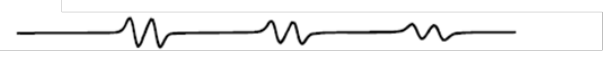

Simulation of trichloromethyl-PBN

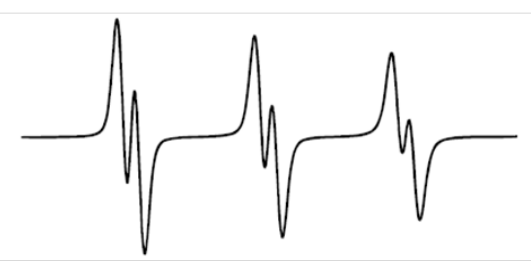

Difference between experimenal and simulated spectra

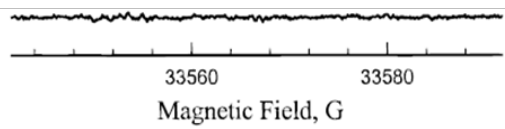

Figure 1-21. Detection of two radical species at low and high frequencies. A) $1^{\text {st }}$ derivative X-band EPR spectrum from a mixture of phenyl-PBN and trichloromethyl-PBN radical adducts. The carboncentered spin adducts have close g-values $\left(\Delta \mathrm{g}_{\text {iso }}=1.2 \times 10^{-4}\right)$ and nitrogen hyperfine couplings what leads to an overlap of these two radical species at X-band frequency. B) A measurement of the same 
mixture at W-band shows the presence of two radical adducts as demonstrated through separate simulations of the two radical adducts phenyl-PBN and trichloromethyl-PBN.

The second study shows the impact of high-frequency EPR spectroscopy on the disentanglement of g-values of the radical pair $\mathrm{P}_{865}{ }^{\circ+} \mathrm{Q}_{A}{ }^{-1}$ in frozen solution of the Zn-substituted deuterated RCs from Rb.sphaeroides. Although at $95 \mathrm{GHz}$ one can resolve some g-values of the radical pair only at $360 \mathrm{GHz}$ all g-value components with a g-anisotropy of 0.6 and $2.2 \%$ for $\mathrm{P}_{865}{ }^{\circ+}$ and $\mathrm{Q}_{\mathrm{A}}{ }^{\circ-}$ respectively get completely resolved. Especially in the high-field region where the $g_{z}$-value of the two radicals is nearly the same one can observe the advantage in going to very high frequencies like $360 \mathrm{GHz}$ (Figure 1-22).

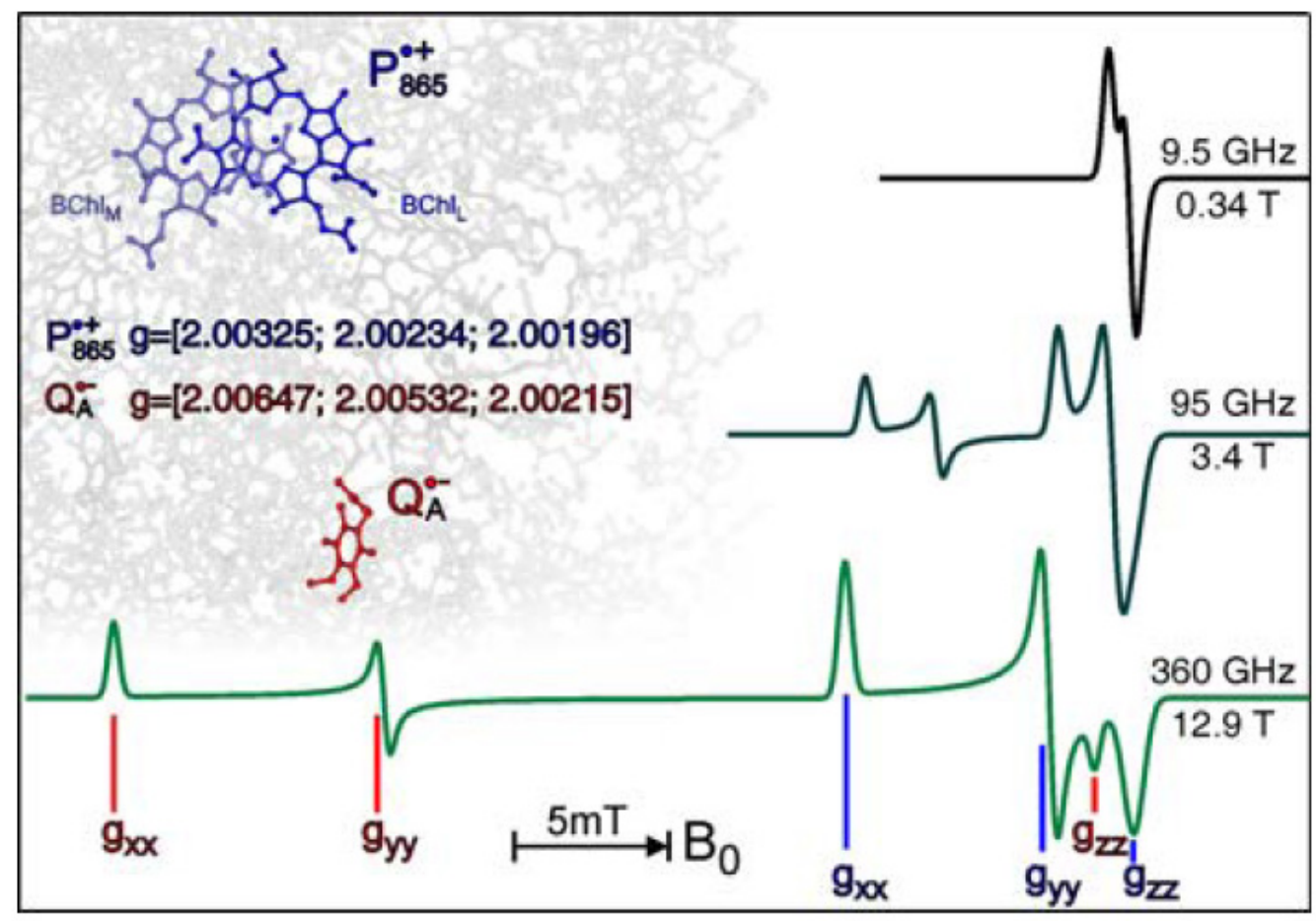

Figure 1-22. First derivative CW EPR spectra of $P_{865}{ }^{\circ+}$ and $Q_{A}{ }^{\circ-}$ for the frequencies $9.5,95$ and 360 $\mathrm{GHz}$. All EPR components for both radical species are completely resolved at $360 \mathrm{GHz}$. The inset shows the corresponding g-values as well as the cofactor arrangement according to X-ray crystallography (84). 
In the last study three radical species in the E.coli RNR E441Q $\alpha$-mutant could be disentangled at $140 \mathrm{GHz}$ (32). One could separate hereby the signals of a disulfide anion radical, an organic carbon-centered radical and the persistent $\mathrm{Y}_{122}{ }^{\circ}$ in the $\beta$-subunit (Figure 1-23). At $10 \mathrm{~K}$ the spectrum of the stable $Y_{122}{ }^{\bullet}$ is visible together with a very weak tail of a second radical species at the low field side (see $20 \mathrm{x}$ amplification). To suppress the strong signal of $Y_{122}{ }^{\circ}$ measurements were performed at 60 or $70 \mathrm{~K}$. Due to the fast $T_{1}$ and $T_{2}$ relaxation at this temperature the signal of $Y_{122}{ }^{\circ}$ is not more detectable and the other two radical species become visible.

Two of the three g-values $\left(g_{x}\right.$ and $g_{y}$ ) of the orthorhombic tensor could be identified to correspond to a disulfide anion radical whereas the third g-value $\left(g_{z}\right)$ of the anion radical lies beneath the signal of an organic carbon-centered radical with much smaller g-anisotropy (see Figure $C$ in Figure 1-23). One can make the $g_{z}$-value visible (see Figure $E$ in Figure 1-23) through an EPR measurement after 3 min reaction time. At this time only the organic radical is detectable as the disulfide anion radical exists on the lower second time scale (10 sec). For the identification of this new protein radical species which was proposed to be on the nucleotide-reduction pathway Lawrence et al. incubated the E441Q RNR mutant with CDP/ATP as substrate and effector.

The cysteine based radical species has been proposed through X-band studies to be the $\mathrm{C}_{439}$ thiyl radical which is on the PCET pathway (85). However the clear assignment of the g-values at $140 \mathrm{GHz}$ proved this analysis to be wrong and showed through comparison of g-values with different sulfur based radical species that the protein radical is indeed a disulfide radical anion which is formed through oxidation of $C_{225}$ and $C_{462}$ in the $\alpha$-subunit. The organic carbon-centered radical has been shown to be a nucleotide based radical, which is not on the normal nucleotide-reduction pathway based on a combined approach of EPR spectroscopy from 9 to $140 \mathrm{GHz}$ together with ENDOR spectroscopy and DFT calculations (31). 


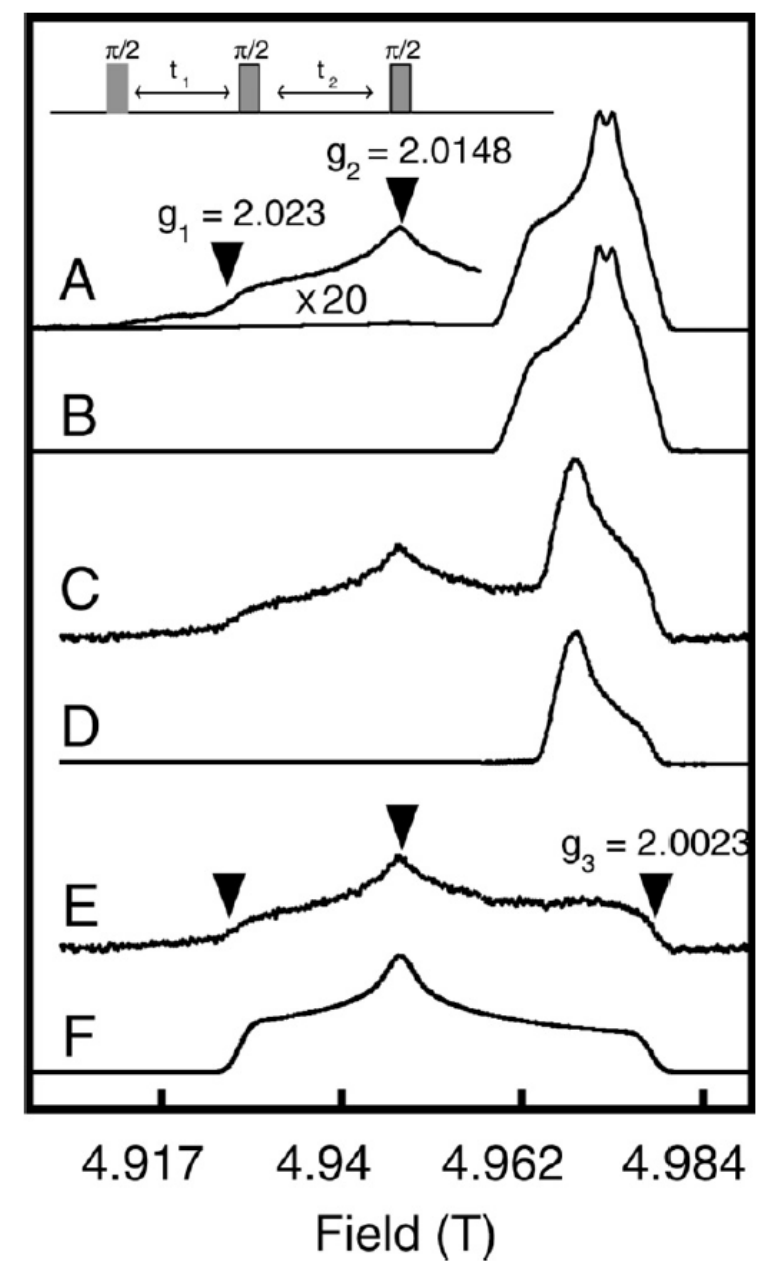

Figure 1-23. Stimulated echo-detected EPR spectra at $140 \mathrm{GHz}$ of the E441Q RNR reaction (the three pulse sequence is shown in the inset). The stimulated echo-sequence is shown in the inset.

A) Spectra at $10 \mathrm{~K}$ after $10 \mathrm{sec}$ quenching time in liquid $\mathrm{N}_{2}$. B) Reference spectrum of $\operatorname{Tyr}_{122}{ }^{\circ}$.

C) Spectrum recorded at $60 \mathrm{~K}$. D) Reference spectrum at $60 \mathrm{~K}$ after 3 min quenching time in liquid $\mathrm{N}_{2}$.

E) Difference spectrum $(C-D)$ representing the disulfide radical anion with the g-values $g_{x}=2.0230$, $\mathrm{g}_{\mathrm{y}}=2.0148$ and $\mathrm{g}_{\mathrm{z}}=2.0023$. F) Simulation of the disulfide radical anion with g-values for $\mathrm{RCS}_{2} \mathrm{~S}^{\circ}-$ $\mathrm{SCH}_{2} \mathrm{R}^{\prime}$ from disulfide radical anion models with $\mathrm{g}_{\mathrm{x}}=2.017-2.024, \mathrm{~g}_{\mathrm{y}}=2.014-2.020$ and $\mathrm{g}_{\mathrm{z}}=2.002$.

In summary the pulsed EPR technique applied at high-fields has been proven to be the ideal strategy in the characterization of three organic radicals with superimposed g-values hereby taking advantage of different radical stabilities. 
Nowadays also a multifrequency approach has been found to be most appropriate to acquire a more complete knowledge of the interactions of a paramagnetic species and its environment. The most frequently used frequency bands in EPR have been displayed in Table 1-1 although multifrequency EPR spectroscopy covers today the range from 0.1 to $1200 \mathrm{GHz}$.

It was noted by Hyde and Froncisz, that at the minimum three frequencies are required for an unambigous picture of an unknown radical species (86). For example if one wants to distinguish between field-dependent and field-independent processes like the electron Zeeman and the hyperfine interaction one can go to particular frequencies. g-values for organic radicals are extracted with highest accuracy at high frequencies. Depending on the ganisotropy $\left(\Delta \mathrm{g} / \mathrm{g}_{\text {iso }}\right)$ microwave frequencies of $180 \mathrm{GHz}$ are perfectly enough to extract g-values. In some cases one has to go to more than $300 \mathrm{GHz}$ in order to resolve very small g-anisotropies. In general a rule of thumb is that to resolve g-anisotropies which are less than $1 \% 90 \mathrm{GHz}$ or higher are enough. On the other hand the hyperfine interaction dominates at lower frequencies and can be analyzed in the best way at $9 \mathrm{GHz}$. By choosing a particular frequency it is therefore possible to study a particular process in the EPR spectrum.

However we have chosen in our multifrequency characterization of the $\mathrm{NH}_{2} \mathrm{Y}^{\bullet}$ three EPR frequencies: 9,94 and $180 \mathrm{GHz}$. At the two frequencies 9 and $94 \mathrm{GHz}$ the hyperfine interaction is dominating and the A-tensor or hyperfine tensor is best determined at these frequencies. One can determine from $9 \mathrm{GHz}$ spectra the hyperfine interactions more accurately than from $94 \mathrm{GHz}$ spectra. Still the medium frequency is ideal to check for consistency of the hyperfine and Zeeman interaction together, whereas the $9 \mathrm{GHz}$ and 180 $\mathrm{GHz}$ spectrum are sensitive to only one type of interaction. For example at $180 \mathrm{GHz}$ the intrinsic linewidth is around $25-30 \mathrm{MHz}$. This means that one is able to resolve only large hyperfine couplings for the $\mathrm{NH}_{2} \mathrm{Y}^{\bullet}$ species at position $\beta-356, \alpha-731$ and $\alpha-730$ (see Chapter 3 Table 3-1). However at $180 \mathrm{GHz}$ one could easily determine g-values of the $\mathrm{NH}_{2} \mathrm{Y}^{\bullet}$ (see Chapter 3 Figure 3-1).

Another usage of the multifrequency approach is to characterize different motional regimes of a spin labeled macromolecule. As briefly mentioned above on MTSL labeled T4 lysozyme the local motion of the label was studied at $170 \mathrm{GHz}$ (picoseconds to nanoseconds time regime) whereas at $9 \mathrm{GHz}$ EPR is more sensitive to slow motions like the overall motion of a 
macromolecule (microsecond to second time regime). This means that the spin label can be indirectly used to get out information about the dynamics of the spin-labeled macromolecule.

Two of the major usages of the multifrequency EPR approach have been demonstrated here, although there are also other additional insights one can get out from multifrequency EPR spectroscopy i.e. the identification of specific relaxation mechanisms as some of the $T_{1}$ relaxation mechanisms like the thermally activated once are frequency dependent.

To summarize multifrequency EPR spectroscopy is a very new and powerful approach in the EPR field but requires laboratories with at least two high-field EPR spectrometers, one which is already implemented in our research group $(94 \mathrm{GHz})$ and another one which will be available soon $(260 \mathrm{GHz})$. A general review on multifrequency EPR spectroscopy is until today not available although one can get out information about this field in more specific reviews related to different paramagnetic systems $(87,88)$. 


\subsection{High-field ENDOR spectroscopy and the characterization of hydrogen interactions}

ENDOR is a double resonance method (electron nuclear double resonance) that allows the determination of hyperfine couplings between electron and nuclear spins hereby giving direct insight about their inter-spin distances as well as the nature and number of nuclei coupled to a paramagnetic species (Figure 1-24).

A

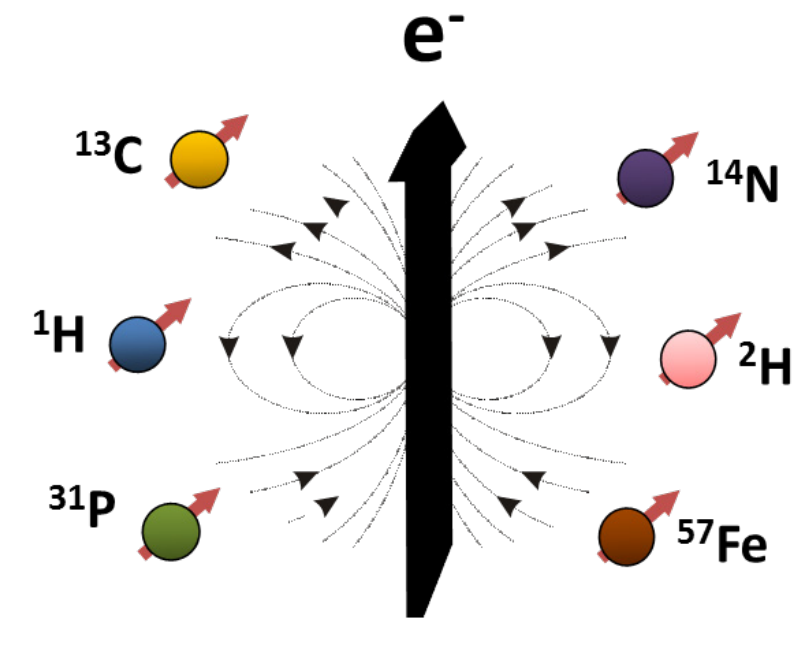

B

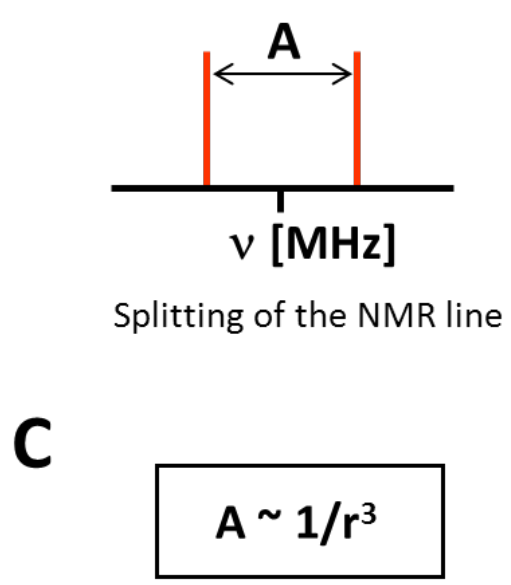

Figure 1-24. Hyperfine interaction between an electron and different nuclei. A) The ENDOR method allows the detection of different nuclei via the electron spin. ${ }^{1} \mathrm{H},{ }^{13} \mathrm{C},{ }^{31} \mathrm{P}$ and ${ }^{57} \mathrm{Fe}$ have a spin of $\mathrm{S}=1 / 2$ and ${ }^{2} \mathrm{H},{ }^{14} \mathrm{~N}$ have a spin of $\mathrm{S}=1$ respectively. B) Splitting of the NMR line due to hyperfine interaction between an electron and a nucleus with a spin of $I=1 / 2$. C) Reciprocal relationship between the hyperfine coupling and the electron-nucleus distance.

When applied at high frequencies (for organic radicals) one can get out additionally besides the distances, the directions of bonds formed between nuclei and the paramagnetic species due to higher g-tensor resolution. Moreover one profits from improved ENDOR resolution with high frequency what allows the separate detection and analysis of different nucleic hyperfine interactions (Figure 1-25). ENDOR spectra at high frequencies facilitate hereby an unambiguous identification and interpretation of a certain nucleus. Additionally to this the 
indirect detection of the NMR spectrum through the electron spin leads in general to much higher sensitivity which can not be achieved through conventional NMR spectroscopy.

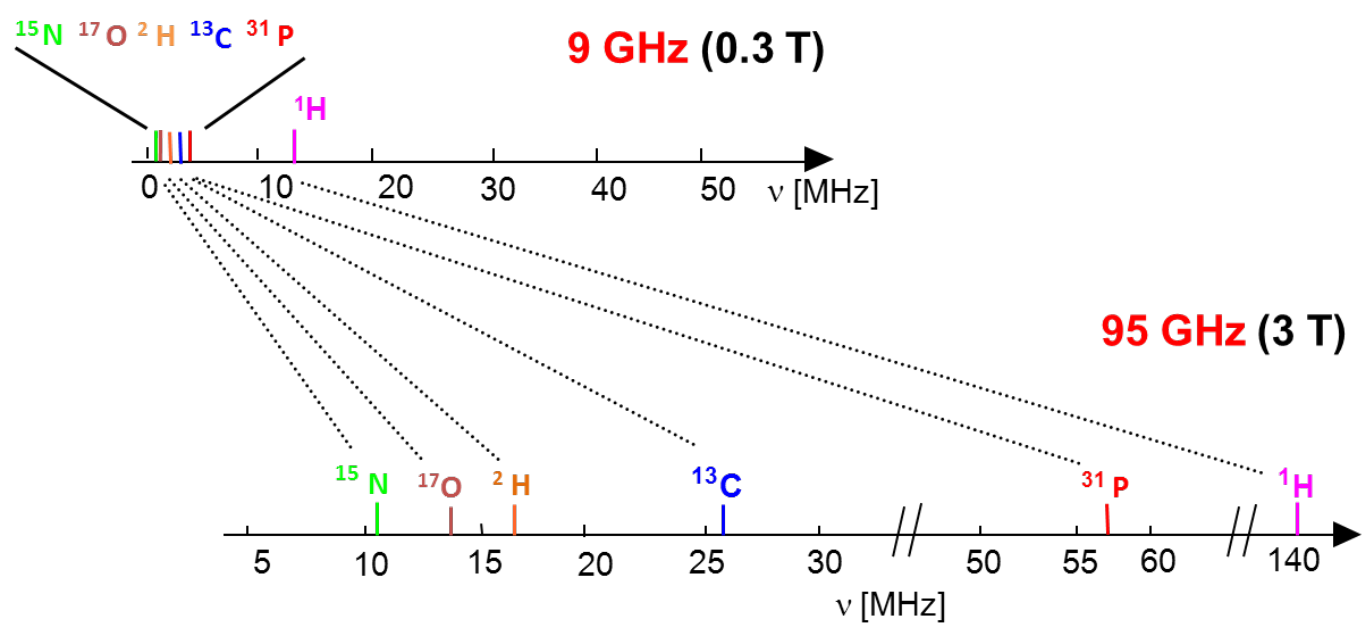

Figure 1-25. Comparison of ENDOR resolution at different fields. At X-band the nucleic resonances for nearly all nuclei coincide. With increasing magnetic field one observes a significant resolution and at $95 \mathrm{GHz}$ and all nucleic resonances appear in a different region of the NMR scale.

The ENDOR method has been introduced by Feher in solid state physics (89) and later it was extended to radicals in solution by Hyde and Maki (90). It is a combination of electron paramagnetic resonance and nuclear magnetic resonance spectroscopy where one applies MW irradiation together with RF irradiation in order to stimulate EPR and NMR transitions. The two types of spectroscopic transitions have to follow hereby different selection rules and can be expressed in an energy level diagram (Figure 1-26). In case of EPR transitions (EPR 1 and EPR 2) the selection rule $\Delta m_{s}= \pm 1$ and $\Delta m_{1}=0$ holds, whereas for NMR transitions (NMR 1 and NMR 2) the rule $\Delta m_{S}=0$ and $\Delta m_{1}= \pm 1$. It should be mentioned that the EPR selection rule is valid for systems with such a hyperfine coupling where the acting hyperfine field on the nucleus is much smaller than the external magnetic field. This is the case when going to frequencies $v \geq 90 \mathrm{GHz}$ and is called the high field limit for electron and nucleus. 


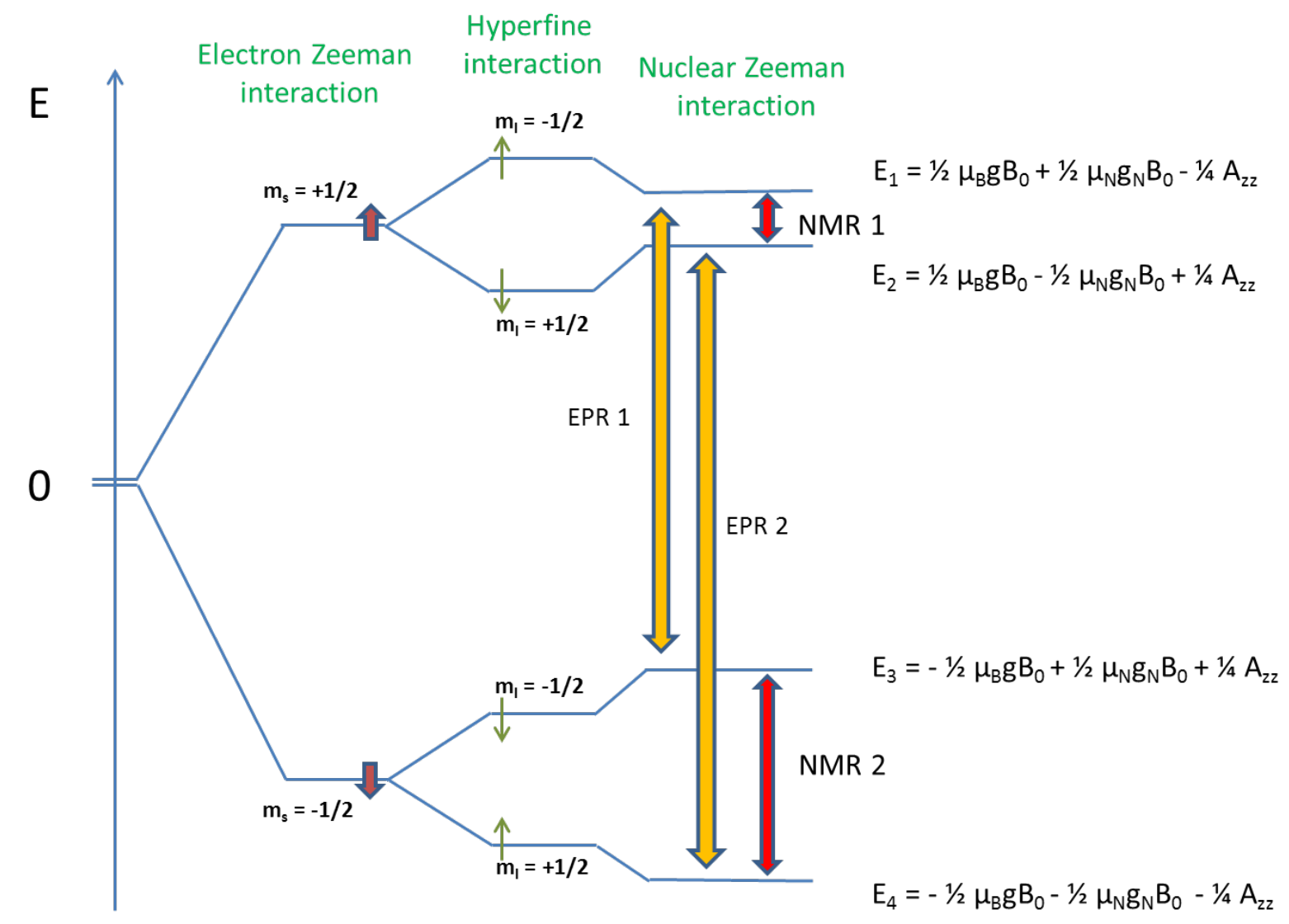

Figure 1-26. Schematic energy level diagram for an electron with $S=1 / 2$ coupled to a nucleus with $I=$ $1 / 2$ in the presence of a magnetic field $B_{0}$. Heavy arrows in red represent the electron spin components along the magnetic field with the corresponding spin quantum number $m_{s}$. The light green arrows represent the nuclear spin components with $m_{1}$ as the spin quantum number. Each electron Zeeman spin level is split into two levels by the hyperfine interaction with the nuclear spin and the hyperfine levels get then shifted by the nuclear Zeeman interaction term. The energy levels $E_{1}$ to $E_{4}$ are expressed as a sum of the electron and nuclear Zeeman as well as the hyperfine interaction. $\mu_{\mathrm{B}}$ and $\mu_{N}$ are the two magnetic moments for electron and nucleus, $g$ and $g_{N}$ are the g-factor for electron and nucleus, $B_{0}$ is the static magnetic field and is the hyperfine interaction along the field direction. Under the high field limit the EPR transitions EPR 1 and EPR 2 are then calculated to be $E_{1}-E_{3}=\mu_{B} g B_{0}$ $-1 / 2 A_{z z}$ and $E_{2}-E_{4}=\mu_{B} g B_{0}+1 / 2 A_{z z}$ respectively and the NMR transitions NMR 1 and NMR 2 are calculated to be $E_{1}-E_{2}=\mu_{N} g_{N} B_{0}-1 / 2 A_{z z}$ and $E_{3}-E_{4}=\mu_{N} g_{N} B_{0}+1 / 2 A_{z z}$.

The ENDOR effect is visible on the EPR line and appears when both the microwave and RF fields are in resonance with the EPR and NMR transitions. In terms of an energy level diagram it means that one EPR transition is irradiated by mw irradiation simultaneously with 
one NMR transition irradiated by the $\mathrm{rf}$ field. One notices then the ENDOR effect in the change of the EPR signal intensity (Figure 1-27).

$\mathbf{E}$

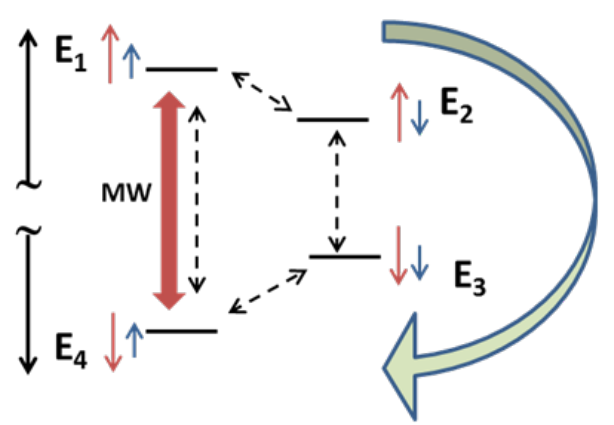

Only spin relaxation
E

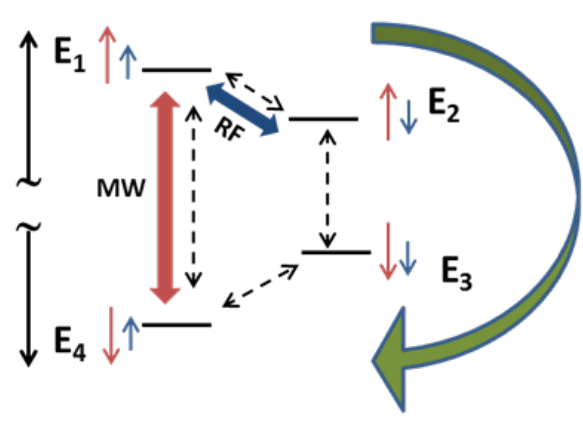

Spin relaxation + NMR transition

\section{Saturated EPR line}

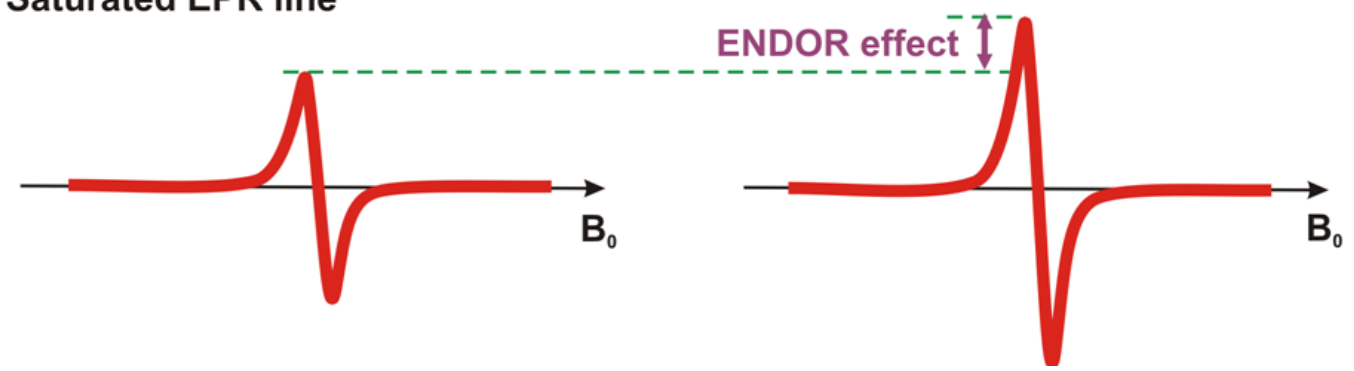

Figure 1-27. Principles of ENDOR spectroscopy. (Left) The energy levels of an $S=1 / 2, I=1 / 2$ spin system in a magnetic field. The EPR transition $1 \leftrightarrow 4$ is partially saturated (level 1 is never depopulated during CW irradiation) as indicated by the vertical arrow in red and the weakened EPR signal is shown below. The various spin relaxation steps are indicated by the dotted arrows and the whole relaxation process between levels 1 and 4 is indicated by the circular arrow which is too slow to recover the population difference. (Right) The NMR transition $1 \leftrightarrow 2$ is induced by the RF irradiation and its effect is the speeding up of the relaxation path $1 \leftrightarrow 2 \leftrightarrow 3 \leftrightarrow 4$, increasing the rate of the population recovery between levels 1 and 4 with desaturation of the EPR line and increase of the intensity. During an ENDOR experiment the EPR signal is measured at a fixed magnetic field, and its intensity is varied by the applied scanned radio frequency irradiation.

Performing ENDOR means to observe in the NMR dimension couplings of specific nuclei to an electron spin via the dipolar hyperfine interaction from which one can derive the distance between an electron and a nucleus and their orientation with respect to the applied magnetic field (Figure 1-28). 


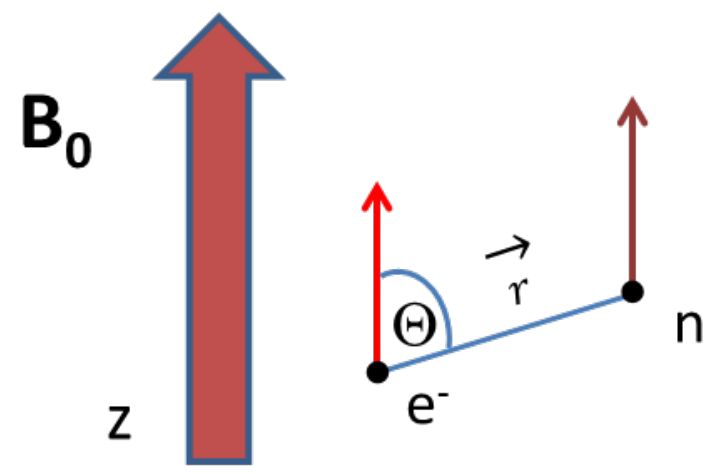

Figure 1-28. Dipole-dipole interaction between a nucleus and an electron in the static magnetic field $\mathrm{B}_{0} . \underset{r}{\rightarrow}$ is the interconnecting vector between the two dipoles and $\Theta$ is the angle between vector $\underset{r}{\rightarrow}$ and the z-axis of $\mathrm{B}_{0}$.

The energy of the hyperfine interaction is given hereby in one term of the spin Hamiltonian that describes the coupling of an electron spin to one or more nuclei $i$. Besides the first and second terms which correspond to the Zeeman interaction of electron and nucleus, the third one describes the hyperfine interaction (Equation 1-5).

$$
\widehat{H}=\mu_{B} \mathbf{S} \cdot \mathbf{g B}+\sum_{i}\left(\boldsymbol{g}_{N}^{i} \mu_{N}^{i} \mathbf{I} \cdot \mathbf{B}\right)+\sum_{i}\left(\mathbf{I} \cdot \boldsymbol{A}^{i} \cdot \mathbf{S}\right)
$$

Equation 1-5. Spin Hamiltonian for an electron spin coupled to a nucleus $(S=1 / 2$ and $I=1 / 2) \cdot \mu_{B}$ and $\mu_{N}^{i}$ are the electronic and nucleic magnetons, $g_{e}$ and $g_{n}^{i}$ the electronic and nucleic g-factors and $\mathbf{S}$ and $\mathbf{I}$ the spin vectors for an electron and a nucleus. $B$ is the static magnetic field and $A^{i}$ the hyperfine interaction.

The hyperfine interaction $A^{i}$ is hereby composed of an isotropic part $\mathrm{a}_{\mathrm{iso}}$ and an anisotropic part or dipolar part (Equation 1-6).

$$
\boldsymbol{A}=a_{i s o}+\left(\begin{array}{ccc}
T_{x x} & T_{x y} & T_{x z} \\
T_{y x} & T_{y y} & T_{y z} \\
T_{z x} & T_{z y} & T_{z z}
\end{array}\right)=\left(\begin{array}{ccc}
A_{x x} & A_{x y} & A_{x z} \\
A_{y x} & A_{y y} & A_{y z} \\
A_{z x} & A_{z y} & A_{z z}
\end{array}\right)
$$


Equation 1-6. The hyperfine interaction is expressed as a sum of a scalar and a tensor ( $3 \times 3$ matrix) in the laboratory frame.

When the dipolar tensor is expressed in its principal axes system, then the matrix representation is diagonal with elements given by the principal axes values (Equation 1-7). For an axially symmetric tensor the diagonal elements $T_{x x}, T_{y y}$ are replaced by $T_{\perp}$ and $T_{z z}$ by $\mathrm{T}_{\|}$and the tensor is called to be traceless when fulfilling the condition $\mathrm{T}_{\|}=-2 \bullet T_{\perp}$.

$$
\mathrm{T}(\operatorname{diag})=\left(\begin{array}{lll}
T_{\perp} & & \\
& T_{\perp} & \\
& & T_{\|}
\end{array}\right)
$$

Equation 1-7. Diagonalized dipolar tensor with its principal values $T_{\|}$and $T_{\perp}$.

In order to calculate the point-dipole distance between electron and nucleus one uses then $T_{\perp}$ in the point-dipole model formula (Equation 1-8).

$$
T_{\perp}=\frac{g_{e} \cdot g_{n} \cdot \mu_{B} \cdot \mu_{N}}{h \cdot r^{3}}
$$

Equation 1-8. Point-dipole model formula for the calculation of interspin distances with $\mathrm{g}_{\mathrm{e}}$ and $\mathrm{g}_{\mathrm{n}}$ being the electronic and nucleic g-factors and $\mu_{B}$ and $\mu_{N}$ being the electronic and nucleic magnetons. $\mathrm{h}$ is the Planck constant and $\mathrm{r}$ the dipole-dipole distance.

From hyperfine spectra in the solid state (powder patterns) one can get out $T_{\perp}$ either directly or indirectly through applying the formula for $T_{\|}$and $A$ (diag). Three case studies are shown below (Figure 1-29) where the hyperfine transitions are centered at the nuclear Larmor frequency $\left(v_{n}\right)$. 


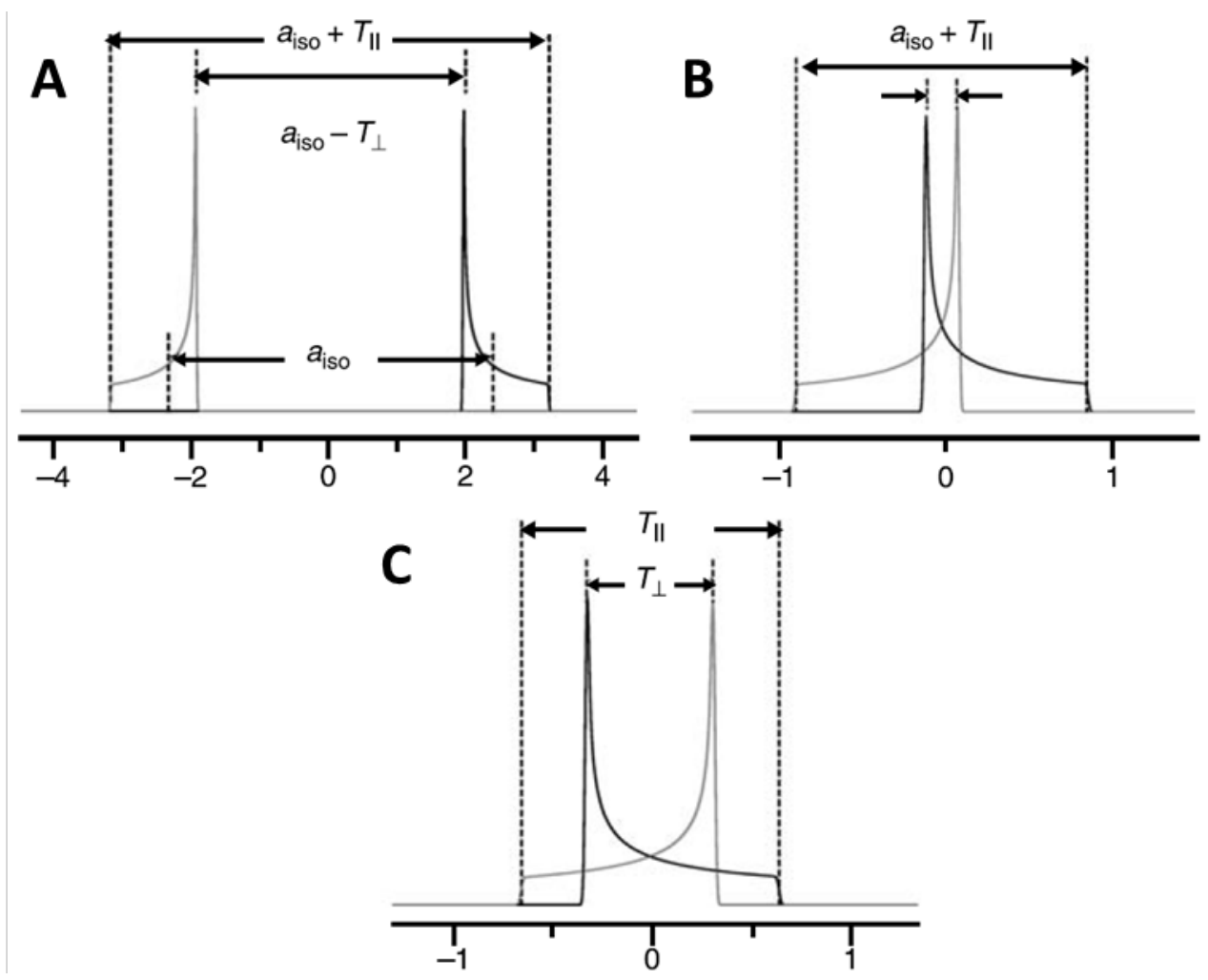

Figure 1-29. Powder patterns for a hyperfine tensor $\mathbf{A}$ with three different examples: $A) a_{\text {iso }}>T$; B) $a_{\text {iso }}<T$ and C) $a_{\text {iso }}=0$. The distributed hyperfine transitions are presented for an $I=1 / 2$ spin coupled to a $S=1 / 2$ spin. The third example is called a Pake pattern and appears through pure dipole dipole interaction which is analogous to the Pake pattern in solid-state NMR.

Besides CW ENDOR there are two relevant methods to do pulsed ENDOR. Mims ENDOR is the first pulsed ENDOR method that was described by Bill Mims in 1965 (91). It consists of a stimulated echo sequence into which a RF pulse is inserted during delay $\mathrm{T}$. When the RF pulse flips a nuclear spin coupled to an electron spin, that electron spin changes resonant frequency by the hyperfine splitting $A$. At a time $\tau$ after the third mw pulse when the stimulated echo appears, the spin will have a phase A. $\tau$ different than without the RF pulse. The refocusing of the spins by the third pulse will be incomplete and the ENDOR signal appears then as a decrease in the intensity of the stimulated echo (Figure 1-30). 


\section{Mims-ENDOR}

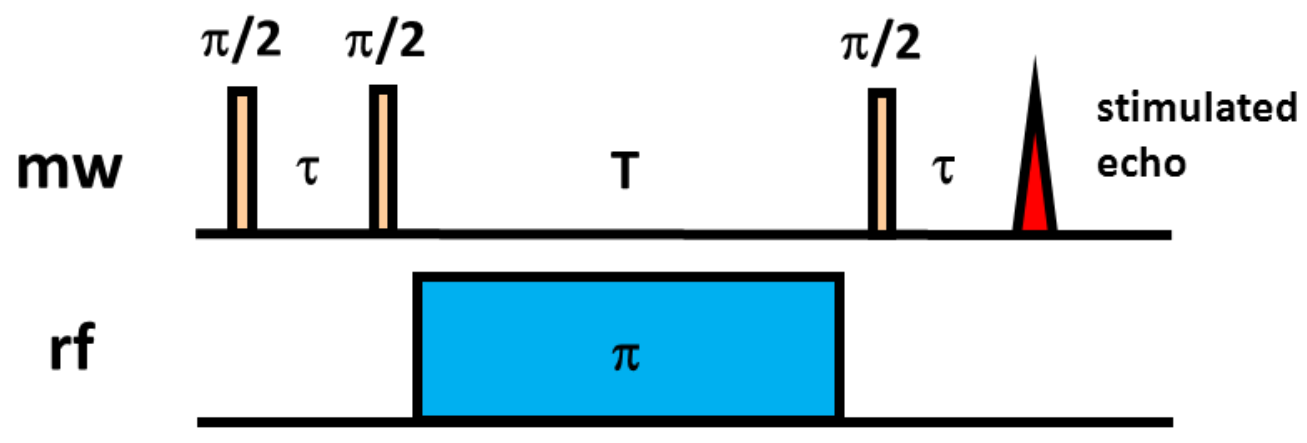

Figure 1-30. The Mims ENDOR sequence consisting the preparation period with the first two mw pulses, the evolution period with delay $\mathrm{T}$ during which the RF pulse is applied, and the detection period followed by the third mw pulse.

The obtained Mims ENDOR spectrum has a periodic modulation with the amplitude oscillating with $\sin ^{2}(2 \cdot \pi \cdot \tau \cdot A)$ hereby producing "blind spots" where no ENDOR signal can be seen (Figure 1-31). A mistake is to account these blind spots for real structure in the ENDOR spectrum. If the $\tau$ between the first and second $\mathrm{mw}$ pulse is too large edges of a spectrum can fall into the blind spot region and if $\tau$ is too small a quite pronounced blind spot around the nuclear Zeeman frequency suppresses the ENDOR signals from small hyperfine couplings although there is always some residual intensity from the matrix ENDOR line. Generally it is necessary to measure spectra at several values of $\tau$ where one uses small $\tau$-values for large couplings and large $\tau$-values for small couplings and explicitly consider the blind spots in the spectral analysis and simulation of ENDOR spectra. Mims ENDOR is most efficient for small couplings $(A<2 \mathrm{MHz}$ ), in which typical $\tau$-values of $150-250$ ns place the blind spots outside the ENDOR spectral region. Therefore this method is preferably used for nuclei with small gyromagnetic ratio $(\gamma)$ like for ${ }^{2} \mathrm{H}$ and ${ }^{13} \mathrm{C}$. 


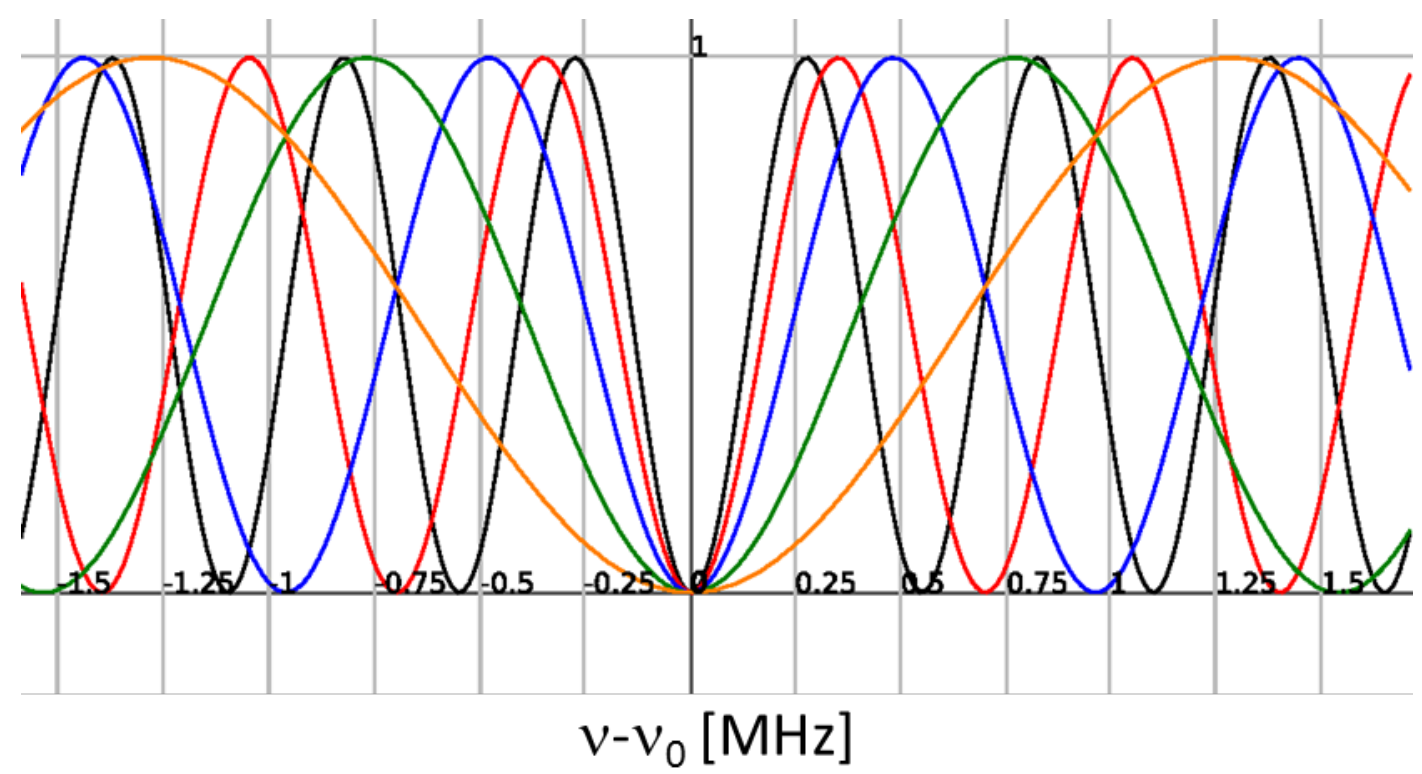

Figure 1-31. The Mims hole function $\left(f(x) \sim \sin ^{2}(2 \cdot \pi \cdot \tau \cdot A)\right)$ for different $\tau$-values with $A$ being $v-v_{0}$ ( $\tau=700 \mathrm{~ns}$ (black); $\tau=550 \mathrm{~ns}$ (red); $\tau=400 \mathrm{~ns}$ (blue); $\tau=250 \mathrm{~ns}$ (green); $\tau=150 \mathrm{~ns}$ (orange)). In case of all $\tau$-values the first blind spot appears at the frequency $v-v_{0}=0 \mathrm{MHz}$. For example the second and third blind spot in case of $\tau=700 \mathrm{~ns}$ appear after $v-v_{0}= \pm 0.55$ and $\pm 1.1 \mathrm{MHz}$.

The second approach to ENDOR is the Davies ENDOR experiment which is the complementary method to Mims ENDOR (Figure 1-32) (92). The method is most suited for systems with medium to large hyperfine couplings $(A>2 \mathrm{MHz})$ as it produces significant strong holes around the nuclear Zeeman frequency. Accordingly it is used in general for the detection of ${ }^{1} \mathrm{H}$ resonances which have a large $\gamma$ which is 6.5 times higher than for ${ }^{2} \mathrm{H}$. The first pulse is called the preparation pulse that inverts the magnetization in a narrow hole near the proton resonant frequency. Afterwards follows a RF pulse which is inserted during the delay $T$ and at the end a $\pi / 2-\pi$ sequence which produces a primary echo from the spins in and around the hole. The ENDOR signal appears as a loss of the inverted hole as the RF is swept through the nuclear resonance frequency. The spectrum does depend strongly on the preparation pulse to the extent that small hyperfine couplings are lost for short preparation pulses that produce broad inverted holes (see Appendix Figure A-1). The placement of the detection window with respect to the center of the echo affects the sensitivity and resolution in the ENDOR spectrum and like Mims ENDOR, the Davies ENDOR spectrum depends on the measurement conditions like the temperature, the RF pulse length and the repetition time. 


\section{Davies-ENDOR}

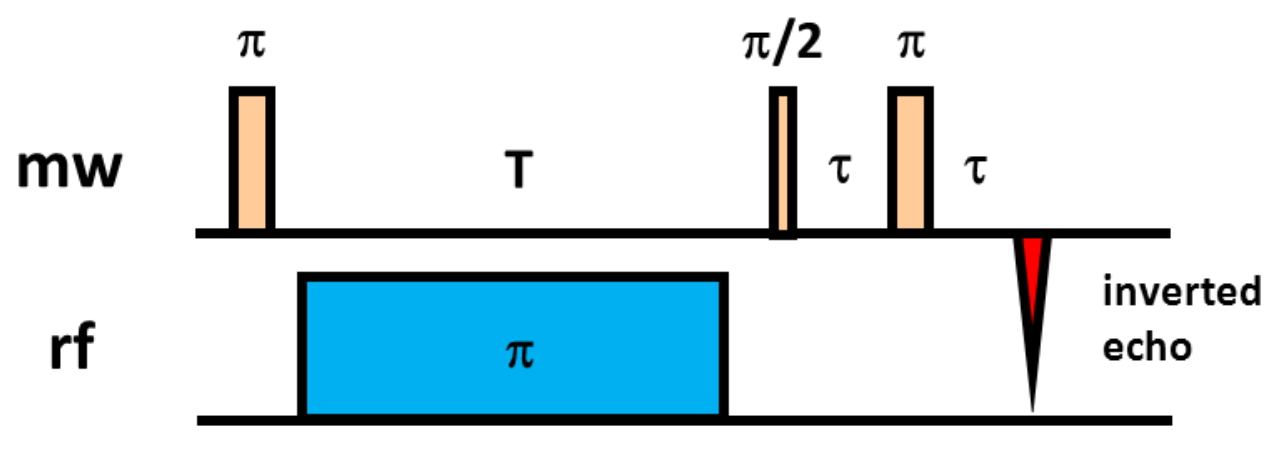

Figure 1-32. The Davies ENDOR sequence consisting the preparation period with one mw pulse, the evolution period with delay T during which the RF pulse is applied, and the detection period followed by two microwave pulses ( $\pi / 2-\pi$ sequence).

When it comes to the detection of nuclei with $\mathrm{I}>1 / 2$ like in case of ${ }^{2} \mathrm{H}$ or ${ }^{14} \mathrm{~N}$ the electric quadrupole moment (Q) of a nucleus has to be taken into consideration. Because of the selection rules for EPR transitions $\Delta \mathrm{m}_{\mathrm{S}}= \pm 1$ and $\Delta \mathrm{m}_{1}=0$ quadrupole effects are not observed in the EPR spectrum but can be observed in the hyperfine structure of an ENDOR spectrum $\left(\Delta \mathrm{m}_{\mathrm{s}}= \pm 1\right.$ and $\left.\Delta \mathrm{m}_{\mathrm{l}}=0\right)$. The interaction between the electric quadrupole moment of a nucleus with the electric field gradients generated by the surrounding electrons induces a coupling which is visible as an additional splitting on hyperfine lines. This scenario is observed when the nuclear Zeeman and the hyperfine interaction are greater than the quadrupole interaction, what is the case for ${ }^{2} \mathrm{H}$ and ${ }^{14} \mathrm{~N}$ (Figure 1-33).

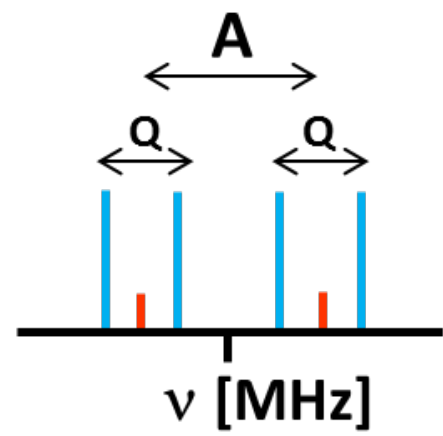

\section{Splitting of the NMR line and the hyperfine line}


Figure 1-33. Doublet of doublet splitting pattern due to hyperfine and quadrupole interaction of an electron spin of $S=1 / 2$ with a nuclear spin of $I=1$. The red lines are marks representing the hyperfine interaction.

In terms of energy the quadrupole Hamiltonian (Equation 1-9) has to be added to the already described Hamiltonian in Equation 1-5.

$$
\widehat{H}_{Q}=\boldsymbol{I} \cdot \boldsymbol{Q} \cdot \boldsymbol{I}
$$

Equation 1-9. Quadrupole Hamiltonian describing the quadrupole interaction with I being the nuclear spin vector and $Q$ the quadrupole tensor that is traceless and symmetric (see Equation 1-7).

A descriptive example of a quadrupole effect in a hyperfine spectrum is the orientation selective high-field ENDOR study $(140 \mathrm{GHz})$ by Bar et al. which showed a coupling of a single deuterium nucleus to an unpaired electron (93). The study is reported for a hydrogen-bond interaction in yeast RNR between one deuterium of a $\mathrm{D}_{2} \mathrm{O}$ molecule (protein exchanged in $\mathrm{D}_{2} \mathrm{O}$ buffer) and the oxygen atom of the endogenous tyrosyl radical. As it was mentioned in the high-field EPR section in going to high frequencies one observes additionally orientation selection of the g-tensor which enables to extract the anisotropy of other interactions like the orientation of the dipolar tensor with respect to the g-tensor axis system. The $D_{z z}$-component lies hereby in the bond between the paramagnetic center and nucleus and one can determine the bond length and direction within the g-tensor frame through recording two-dimensional ENDOR spectra. In practice this means that one measures ENDOR spectra at different positions of the EPR spectra using hereby MW pulse bandwidths which are smaller than the total width of the EPR powder pattern. Orientation selective ENDOR could successfully be employed on transition metal ion complexes at X-and Q-band due to large g-anisotropy. However for organic radicals $(\Delta \mathrm{g} / \mathrm{g}<1 \%)$ one needs $\mathrm{MW}$ frequencies which are $94 \mathrm{GHz}$ and higher as it is demonstrated here on one of the first orientation selective studies on the tyrosyl radical in yeast RNR.

The motivation to do this study was the observation of a substantial shift of the $g_{x}$-value to higher fields. A $g_{x}$-value of tyrosyl radicals which is smaller than 2.0080 is indicative for a hydrogen-bond and high-field EPR studies on many different RNR tyrosyl radicals from 62| P a g e 
different organisms lead to a division of class I RNRs into two groups depending on their $\mathrm{g}_{\mathrm{x}}$-values or their g-anisotropy $\left(\Delta \mathrm{g}=\mathrm{g}_{\mathrm{x}}-\mathrm{g}_{\mathrm{z}}\right)$ (see Table 1-2).

\begin{tabular}{|c|c|c|c|c|}
\hline RNR organism & $\mathbf{g}_{\mathbf{x}}$ & $\mathbf{g}_{\mathbf{y}}$ & $\mathbf{g}_{\mathbf{z}}$ & $\Delta \mathbf{g}=\mathbf{g}_{\mathbf{x}}-\mathbf{g}_{\mathbf{z}}$ \\
\hline E.coli & 2.00912 & 2.00457 & 2.00225 & 0.00687 \\
\hline M.tuberculosis & $2.0080-92$ & 2.0046 & 2.0022 & $0.0058-0.0070$ \\
\hline S.typhimurium & 2.00897 & 2.00437 & 2.00217 & 0.0068 \\
\hline Mouse & 2.0076 & 2.0043 & 2.0022 & 0.0054 \\
\hline Yeast & 2.0077 & 2.00435 & 2.00229 & 0.00541 \\
\hline HSV1 & 2.0076 & 2.0043 & 2.0022 & 0.0054 \\
\hline
\end{tabular}

Table 1-2: Summary of principal g-values and g-anisotropy for various tyrosyl radicals from different class I RNR enzyme (94-98). RNR enzymes in grey consist of hydrogen-bonded tyrosyl radicals. The change in g-anisotropy from one organism to the other is quite drastic and it amounts to almost $30 \%$ but in contrast to the $g_{x}$-value the $g_{y}$ and $g_{z}$-values are similar from one tyrosyl radical to the other. In some cases like for M.tuberculosis one observes a distribution of $g_{x}$-values when measuring at very high EPR frequencies of $285 \mathrm{GHz}$.

Here in this Thesis the $g_{x}$-values together with orientation selective ${ }^{2} \mathrm{H}$ Mims ENDOR spectroscopy have been used to characterize the electrostatic and hydrogen-bond environment in E.coli RNR around the unnatural amino acid radical $\mathrm{NH}_{2} \mathrm{Y}^{\circ}$.

In summary the advantages of high-field ENDOR are firstly derived from those of high-field EPR which include:

(a) High sensitivity for size and concentration-limited samples

(b) Large magnetic field spread, permitting better orientation selection for samples with small g-anisotropy

(c) Better resolution of paramagnetic centers with different g-values consequently allowing also the separation of their ENDOR spectra

Further advantages which are unique to (high-field) ENDOR are:

(a) Better frequency separation for nuclei with similar gyromagnetic ratios $(\gamma)$

(b) Detection of quadrupole interactions 
(c) Applicability of the high field limit which facilitates the interpretation of high-field ENDOR spectra

However as the precondition for pulse ENDOR experiments is the requirement of concomitant $\mathrm{MW}$ and RF irradiation at the sample position, one needs a much more demanding setup which is based on pulse EPR with the additional difference that for ENDOR, a RF source and an amplifier is necessary. Another difficulty is the optimization of experimental parameters like RF power and temperature in ENDOR experiments.

Nevertheless in the $140 \mathrm{GHz}$ orientation selective ENDOR study of yeast RNR the spectra have been obtained with a very good signal/noise making an analysis of the ENDOR traces easily possible. Protein samples of $0.5 \mu \mathrm{L}$ and concentrations of $0.5 \mathrm{mM}$ have been used and the measurements were performed at $6 \mathrm{~K}$ with $1 \mathrm{~kW}$ of RF power allowing hereby two scans per spectrum with 100 shots/point. The high-field ENDOR characterization of the $\mathrm{NH}_{2} \mathrm{Ys}^{\bullet}$ was performed with similar conditions but with a ten times lower radical concentration resulting in experiments with much higher time consumption.

In the introduction two current frontiers of EPR research have been presented: high-field EPR and ENDOR. The impact of these two methods in the structural elucidation of hydrogen-bonding networks and characterization of new amino acid radicals will be demonstrated in chapters 3 and 4 of this Thesis.

Although X-ray crystallography and NMR spectroscopy are by far the most established methods for the determination of three-dimensional structures, ENDOR at high-field is a true alternative when it comes to the characterization of functional protons in enzymes or enzyme complexes which use radical chemistry.

A prerequisite for the analysis of the PCET pathway in E.coli RNR $\alpha$-subunit by EPR and ENDOR spectroscopy is the incorporation of the unnatural amino acid $\mathrm{NH}_{2} \mathrm{Y}$ which functions as an efficient spin trap. In order to do this the suppressor tRNA/aminoacyl-tRNA synthetase methodology has been used in the laboratory of Prof. JoAnne Stubbe at MIT during a six month period in order to produce significant purified amounts of $\alpha-\mathrm{NH}_{2} \mathrm{Y}$. 
Chapter 2

Site-specific incorporation of 3-aminotyrosine into the E.coli RNR $\alpha$-subunit using an evolved suppressor tRNA/tRNA-synthetase pair 


\subsection{Introduction}

The unavailability of natural amino acids that can perturb the reduction potential or protonation states of redox-active $\mathrm{Y}$ or $\mathrm{W}$ residues has limited the usefulness of site-directed mutagenesis methods to probe the radical propagation mechanism in E.coli RNR. However the substitution of $Y_{730}$ and $Y_{731}$ through phenylalanine demonstrated the inactivity of the enzyme but precluded mechanistic investigations of the two $\alpha 2$ amino acid residues (1-3). To examine the pathway for radical propagation as well as the requirements for PCET, we report in this chapter the in vivo usage of the previously evolved $M$. jannaschii aminoacyltRNA synthetase $\left(\mathrm{NH}_{2} \mathrm{Y}-\mathrm{RS}\right)$ with the appropriate $M$. jannaschii amber suppressor tRNA (mutRNA $A_{C U A}$ ) for site-specific incorporation of $\mathrm{NH}_{2} \mathrm{Y}$ (3-aminotyrosine) at position 730 and 731 in a2 (Figure 2-1) (4-7).

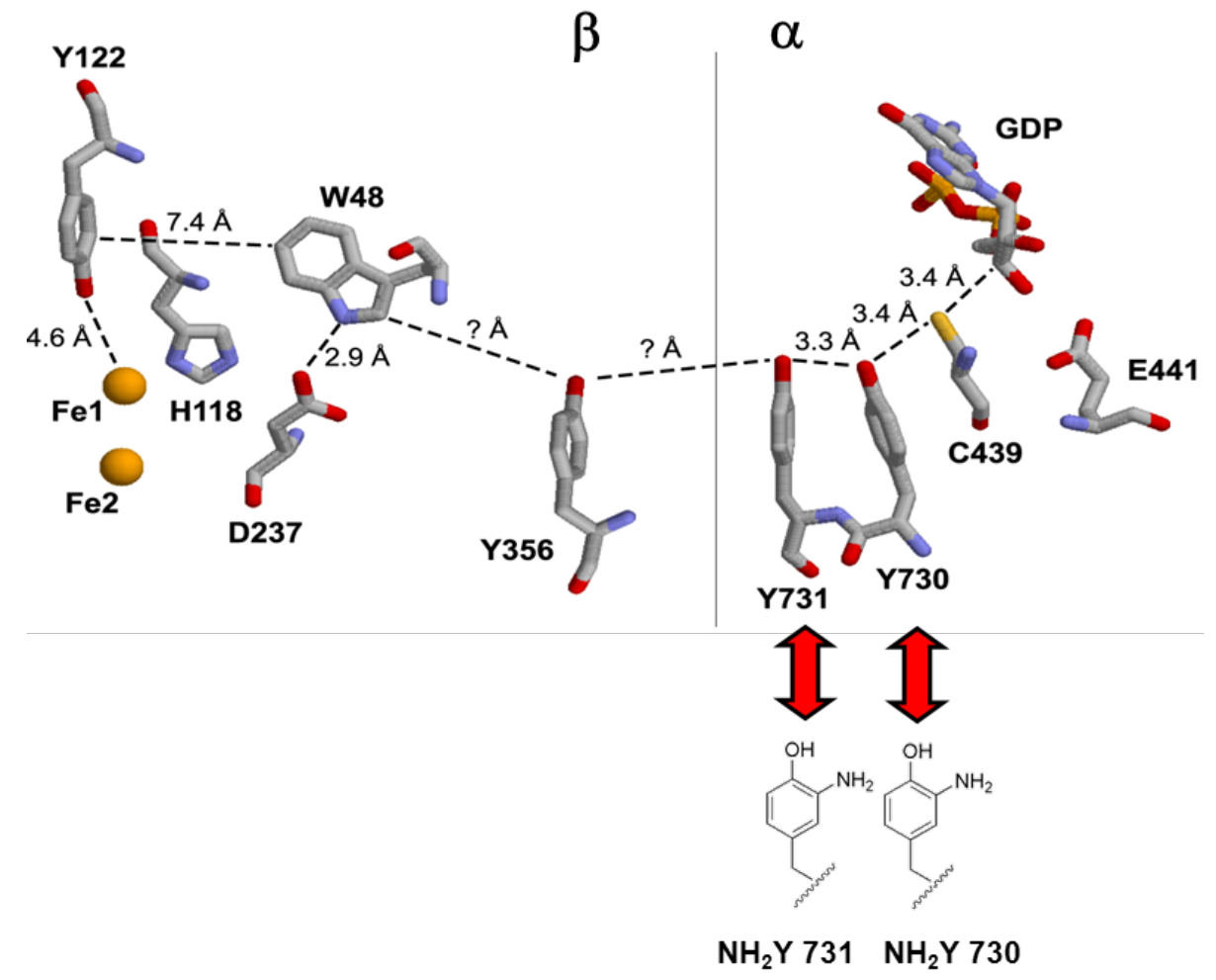

Figure 2-1. The proposed radical transfer pathway of E.coli RNR showing $\mathrm{NH}_{2} \mathrm{Y}$ substitutions of $\mathrm{Y}_{730}$ and $Y_{731}$ in $\alpha 2$. $Y_{356}$ from the $\beta$-subunit is not observable in any of the crystal structures. Therefore, the distances from $Y_{356}$ to $\beta-W_{48}$ and to $\alpha-Y_{731}$ are unknown. Distances in the $\alpha 2$ subunit result from the crystal structure on wild type determined by Uhlin and Eklund and are shown to be minimally perturbed after single substitutions of $Y_{730}$ and $Y_{731}$ through $\mathrm{NH}_{2} \mathrm{Y}(8)$. 
$\mathrm{NH}_{2} \mathrm{Y}$ is structurally similar to $\mathrm{Y}$ with a similar phenolic $\mathrm{pK}_{\mathrm{a}}$ but exhibits a lower redox potential $\left(E^{\mathrm{o}^{\prime}}=0.64 \mathrm{~V}\right.$ vs NHE) than $\mathrm{Y}\left(\mathrm{E}^{\mathrm{o}^{\prime}}=0.83 \mathrm{~V}\right.$ vs NHE$)$ at $\mathrm{pH} 7.0$ and has the ability to function as a thermodynamic spin trap during radical propagation (Figure 2-2). Although DOPA is a much more powerful spin trap $\left(E^{0^{\prime}}=0.57 \mathrm{~V}\right.$ vs NHE) it is less stable to the second oxidation step $(9,10)$.<smiles>CCCc1ccc(O)c(N)c1</smiles>

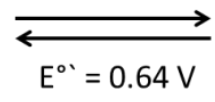<smiles>CCCc1ccc(O)c(N)c1</smiles>

Figure 2-2. One electron oxidation of $\mathrm{NH}_{2} \mathrm{Y}$ with a redox potential of $0.64 \mathrm{~V}$.

The selection process for the isolation of the specific $\mathrm{NH}_{2} \mathrm{Y}$-RS which should charge only the mutRNA $A_{C U A}$ with $\mathrm{NH}_{2} \mathrm{Y}$ during $\mathrm{NH}_{2} \mathrm{Y}_{730}-\alpha 2$ and $\mathrm{NH}_{2} \mathrm{Y}_{731}-\alpha 2$ expression is shown below (Figure 2-3). The directed evolution procedure is meanwhile widely applied and optimized in different laboratories and will be briefly introduced for the selection of the $\mathrm{NH}_{2} \mathrm{Y}-\mathrm{RS}$. The protocol consists of a positive and negative selection step starting with an already selected mutRNA 

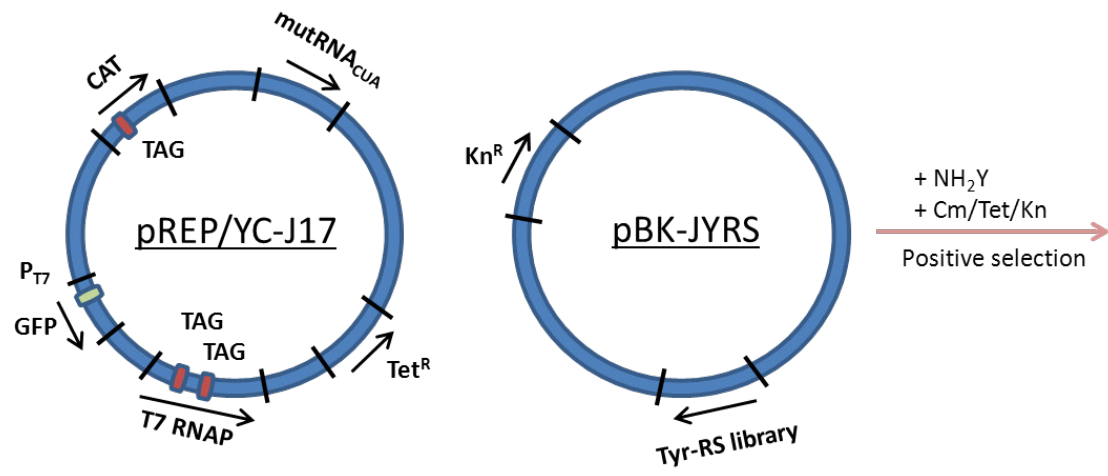

Survivors carry RSs that charge mutRNA ${ }_{\text {CUA }}$ with $\mathrm{NH}_{2} \mathrm{Y}$ or a natural amino acid

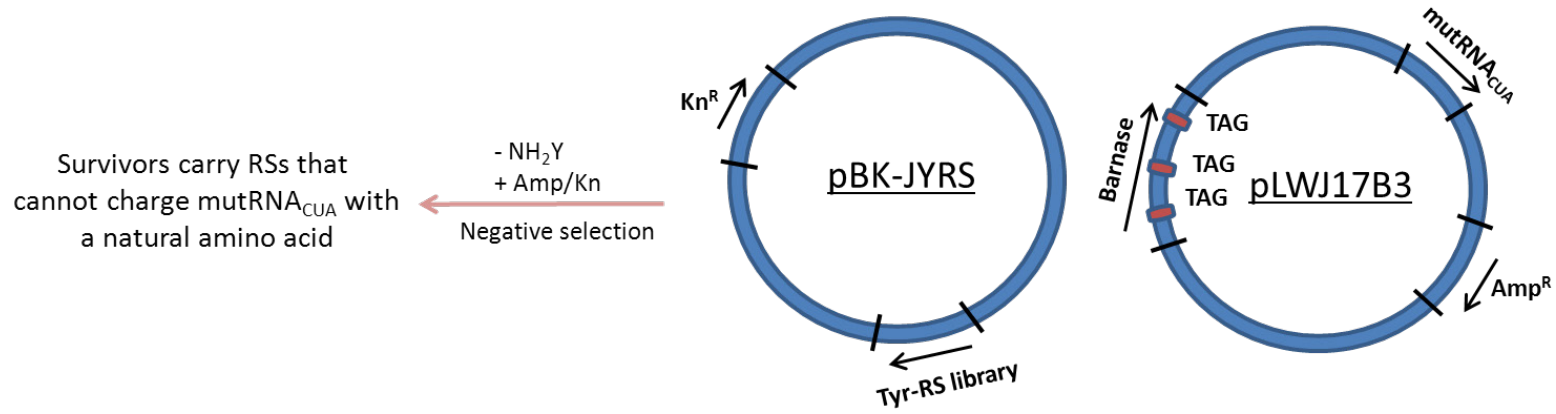

Figure 2-3. General scheme for selection of $\mathrm{NH}_{2} \mathrm{Y}-\mathrm{RS}$. The positive selection is shown in the upper part leading to a survival of RSs that charge mutRNA $\mathrm{CUA}_{\mathrm{A}}$ with $\mathrm{NH}_{2} \mathrm{Y}$ together with natural amino acids of the host. In the lower part the negative selection is shown which eliminates those RSs which charge mutRNA $A_{C U A}$ with natural amino acids. See also text for plasmid description.

The positive selection is performed in $\mathrm{DH} 10 \beta$ E.coli cells containing the positive selection

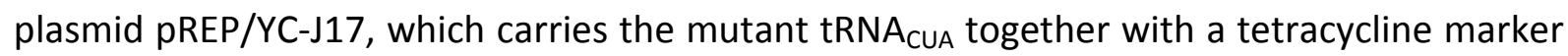
$\left(\right.$ Tet $\left.^{R}\right)$ and the plasmid pBK-JYRS contains the library of RS and a kanamycin marker $\left(\mathrm{Kn}^{\mathrm{R}}\right)$. The positive selection is based on suppression of an amber stop codon (TAG) at a permissive site in the chloramphenicol acetyl transferase (CAT) gene when DH10 $\beta$ E.coli cells are grown in the presence of chloramphenicol $(\mathrm{Cm})$, tetracycline, kanamycin, $\mathrm{NH}_{2} \mathrm{Y}$ and DTT. The positive selection may also be performed by monitoring green fluorescence stemming from GFPuv, which contains a T7 promoter and is expressed when the two TAG codons in the T7 RNA polymerase (RNAP) gene are suppressed. After the first selection RS survive which can charge mutRNA $\mathrm{CUA}_{\text {A }}$ with $\mathrm{NH}_{2} \mathrm{Y}$ or a natural amino acid. The negative selection is based on lack of suppression of three amber stop codons in the barnase gene, which codes for a ribonuclease when the cells are grown in the absence of $\mathrm{NH}_{2} \mathrm{Y}$. The $\mathrm{RS}$ that can charge the suppressor mutRNA $A_{C U A}$ with a natural amino acid generate barnase, which kills the cells. 
After the second selection only RS survive which can charge mutRNA $\mathrm{CUA}_{\text {A }}$ with $\mathrm{NH}_{2} \mathrm{Y}$ but not with a natural amino acid. Four rounds of positive and three of negative selection were required to obtain $\mathrm{RSs}$ that were specific for $\mathrm{NH}_{2} \mathrm{Y}$ and did not cross-react with natural amino acids. The plasmid containing the highly specific RS was designated as $\mathrm{pBK}-\mathrm{NH}_{2} \mathrm{Y}-\mathrm{RS}$.

A rapid test for the efficiency of $\mathrm{NH}_{2} \mathrm{Y}$ incorporation is obtained through MALDI-TOF-MS analysis of the small model protein Z-domain (11) because minor modifications such as an added $\mathrm{NH}_{2}$ group to an amino acid can easily be detected ( $\left.\Delta_{\mathrm{MW}}=15 \mathrm{Da}\right)$. The Z-domain has a mass of $6776 \mathrm{Da}$.

After selection of a $\mathrm{NH}_{2} \mathrm{Y}$-RS which incorporates $\mathrm{NH}_{2} \mathrm{Y}$ with high fidelity and specificity into the $\mathrm{Z}$-domain the same procedure was used to incorporate $\mathrm{NH}_{2} \mathrm{Y}$ within the $\alpha$-subunit with PBAD-nrdA which carries the gene for $\alpha 2$ under the control of an L-Ara-inducible promoter and the mutRNA $\mathrm{CUA}_{\text {g }}$ gene under the control of a Ipp promoter (12). $\mathrm{pBK}-\mathrm{NH}_{2} \mathrm{Y}-\mathrm{RS}$ contains the gene for $\mathrm{NH}_{2} \mathrm{Y}-\mathrm{RS}$ under the control of the E.coli Gln-RS promoter. Although the vector combination gave good yields in case of the Z-domain ( $5 \mathrm{mg}$ per $\mathrm{L}$ cell media) it could not be used for $\alpha 2$ due to low levels of overexpressed $\mathrm{NH}_{2} \mathrm{Y}$ - $\alpha 2 \mathrm{~s}$. The usage of the vector combination was changed to pTrc-nrdA/pAC- $\mathrm{NH}_{2} \mathrm{Y}-\mathrm{RS}$ where the $n r d A$ gene contains an amber stop codon under the control of the highly active trp/lac promoter and the $r r n B$ terminator together with an $A m p^{R}$ marker. $\mathrm{PAC}-\mathrm{NH}_{2} \mathrm{Y}$-RS consists of six genetic copies of mutRNA $\mathrm{A}_{C \mathrm{~A}}$ together with the gene for $\mathrm{NH}_{2} \mathrm{Y}$-RS which is under control of a proK promoter. The combination with pTrc-nrdA lead then to successful overexpressions of $\mathrm{NH}_{2} \mathrm{Y}_{730 / 731}-\alpha 2 \mathrm{~s}$ in similar yields like for the Z-domain.

Finally one has to keep this in mind, while the Z-domain allows for rapid assessment of the quality of the evolved tRNA and RS, the conditions used for optimized expression and suppression of the amber stop codon in the target protein are usually different. 


\subsection{Results}

Unnatural amino acid incorporation into E.coli nitroreductase has been shown to be a successful method through usage of the more active pTrc plasmid (13). Together with the PAC plasmid which carries multiple copies of mutRNA $A_{C U A}$ the concentration of the cognate tRNA and RS could be increased inside the cell (14) and lead to successful expressions of $\mathrm{NH}_{2} \mathrm{Y}_{730 / 731-\alpha 2}$.

A documentation of each specific expression and purification step is shown below in case of wt $\alpha 2$ through SDS PAGE analysis (Figure 2-4 and 2-6).

1

2

3

4

5

6

$7 \quad 8$

9

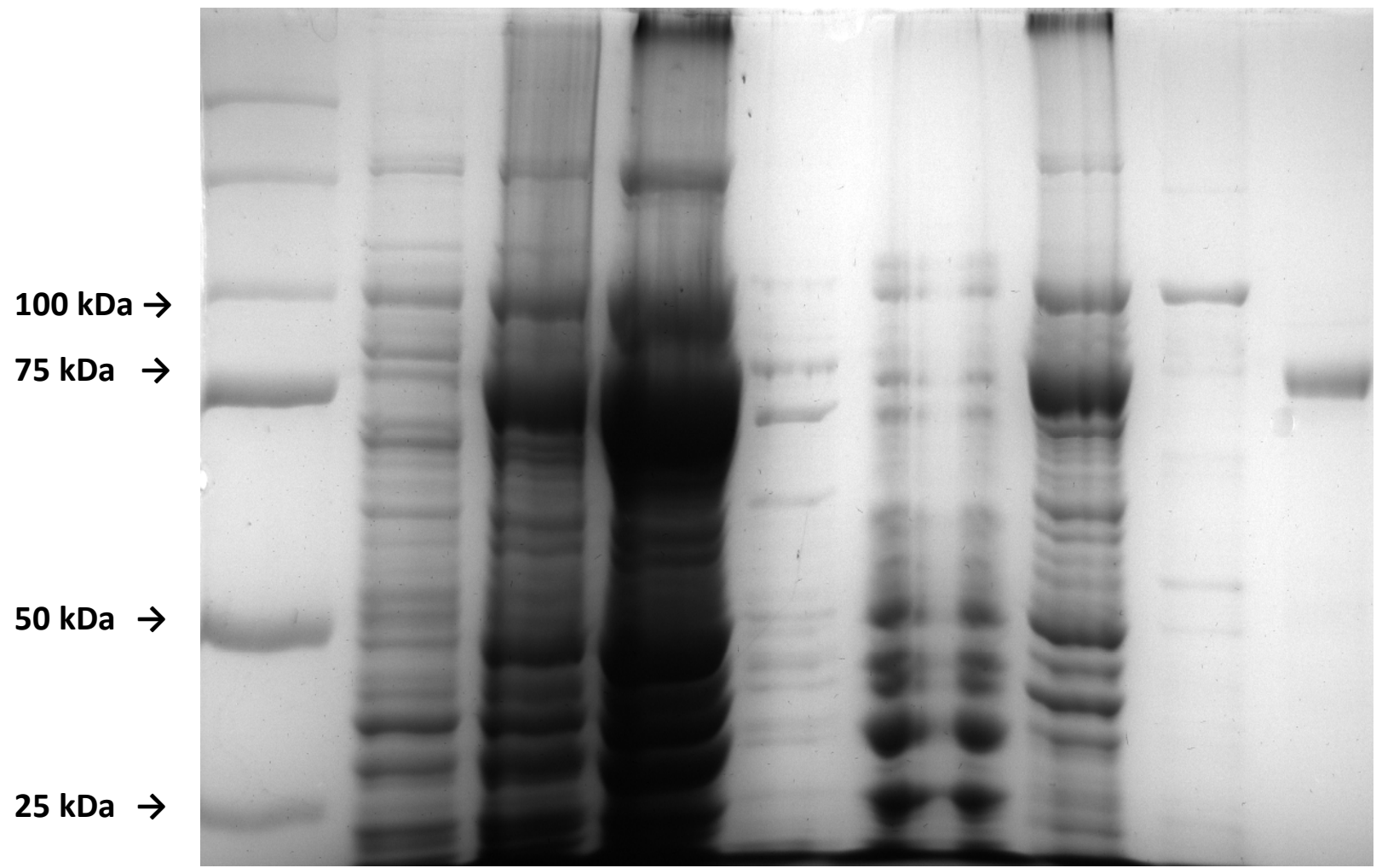

Figure 2-4. SDS PAGE analysis of $\alpha 2$ expression and purification. (1) Molecular weight marker. (2) Before induction with IPTG. (3) After induction with IPTG. (4) Supernatant after lysation. (5) Precipitated fraction after adding streptomycin sulfate. (6) Supernatant after ammonium sulfate precipitation. (7) After G-25 column. (8) DEAE flow through. (9) $\alpha$ monomer standard (85 kDa). 
Expression of wt $\alpha 2$ could be induced through IPTG as it is seen in lane 3 of (Figure 2-4). All purification steps were successful. After double loading brown fractions of the protein onto a DEAE column the column was washed with $\alpha 2$ buffer and $\alpha 2$ was then eluted with a $0.8 \mathrm{x}$ $0.8 \mathrm{~L}$ gradient from $\alpha 2$ buffer to $\alpha 2$ buffer containing $400 \mathrm{mM} \mathrm{NaCl}$. However as it is seen in the elution profile the separation of DNA from proteins was less efficient. Still the fractions from 46 to 59 were collected (Figure 2-5) and were loaded onto a dATP column.

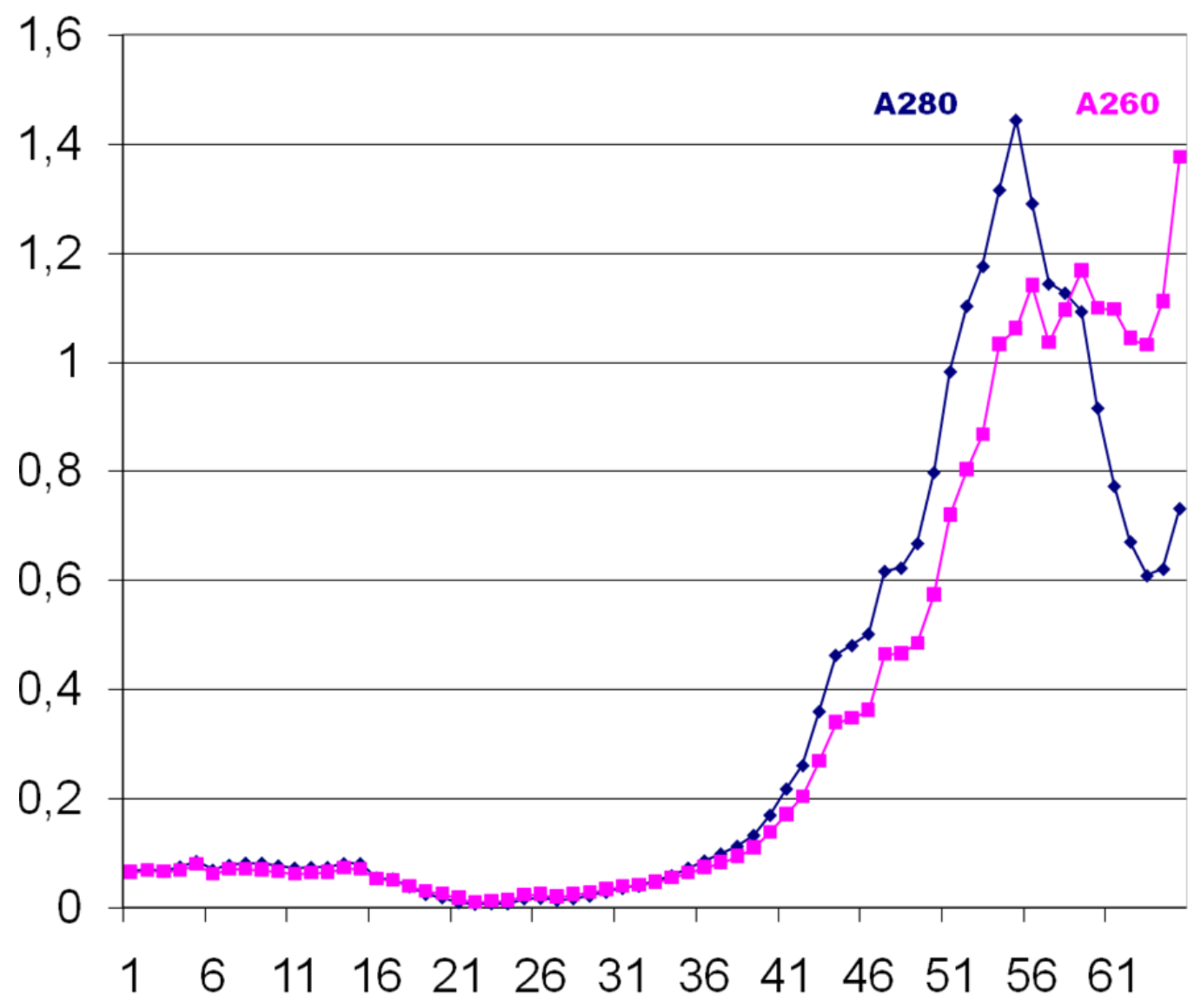

Figure 2-5. DEAE column elution profile. The dependence of the UV/Vis absorbance intensity is shown with respect to the collected column fractions. An absorbance of $280 \mathrm{~nm}$ monitors collected proteins and an absorbance of $260 \mathrm{~nm}$ collected DNA.

The next SDS PAGE analysis shows the successful elution of $\alpha 2$ from the dATP column together with an additional purification with a G25 column. The lanes 1-3 are the same as in Figure 2-4 together with the last lane. In order to quickly check for eluted protein fractions from the dATP column also the Bradford test was used (results not shown). Comparison of 71 | P a g e 
lane 6 with lane 7 demonstrates finally the successful expression and purification of the $\alpha$-monomer.

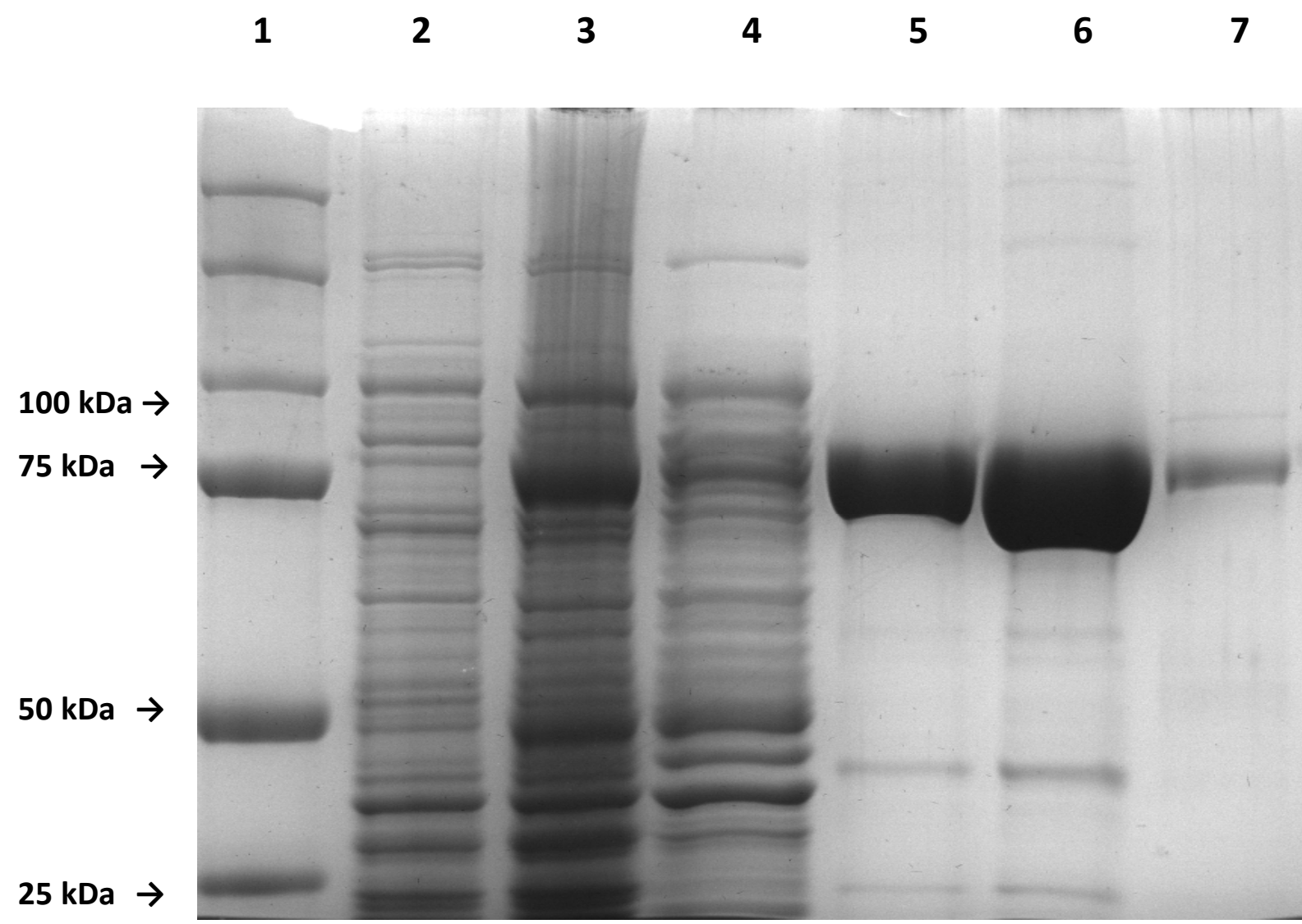

Figure 2-6. SDS PAGE analysis of $\alpha 2$ expression and purification. (1) Molecular weight marker. (2) Before induction. (3) After induction with IPTG. (4) dATP column flow through. (5) $\alpha$ eluted from the dATP column. (6) $\alpha$ after $2^{\text {nd }}$ purification step with G-25 column. (7) $\alpha$ monomer standard ( $85 \mathrm{kDa}$ ).

The mutant proteins $\mathrm{NH}_{2} \mathrm{Y}_{730^{-}}-\alpha 2$ and $\mathrm{NH}_{2} \mathrm{Y}_{731^{-}}-\alpha 2$ behaved similarly during the purification procedure like wt $\alpha 2$ (SDS PAGE not shown). However the DEAE column purification step was skipped as it was possible to bind $\alpha 2$ to the dATP column also in the presence of DNA and finally to elute the desired wt $\alpha 2$. Moreover during the expression of mutant $\alpha 2$ also a truncated form was expressed decreasing hereby substantially the yields of the full length protein. Therefore it was reasonable to skip one of the four purification steps in order to increase the amount of mutant $\alpha 2$. A representative SDS PAGE gel is shown for the expression of $\mathrm{NH}_{2} \mathrm{Y}_{730}-\alpha 2$ and $\mathrm{NH}_{2} \mathrm{Y}_{731}-\alpha 2$ (Figure 2-7). 


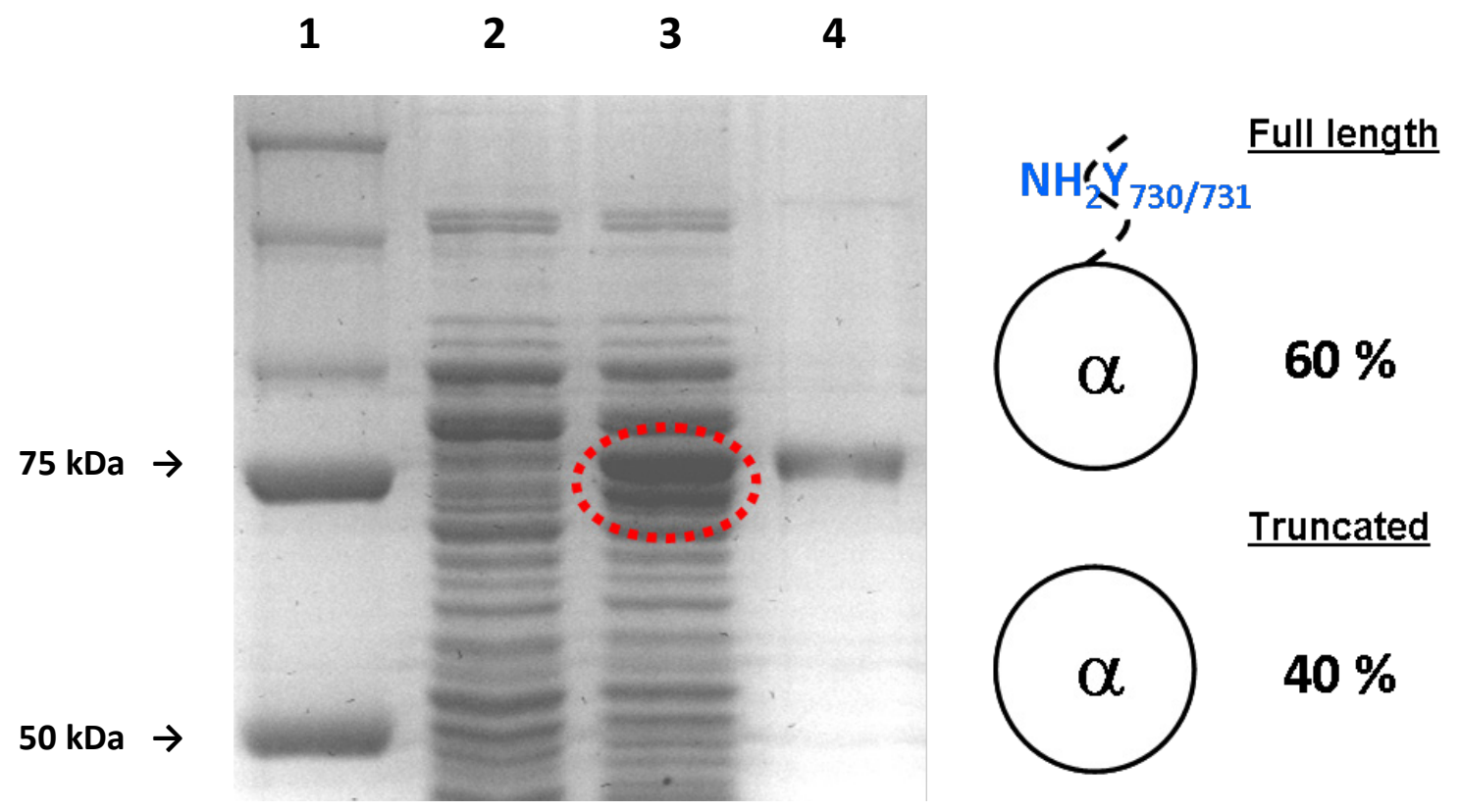

Figure 2-7. Representative SDS PAGE analysis of $\mathrm{NH}_{2} \mathrm{Y}_{730 / 731^{-}}-\alpha 2$ expression. Cells were grown in the absence and presence of IPTG (lane 2 and 3). Lane 1 shows the molecular marker and lane 4 the $\alpha$ monomer standard. The level of full length protein compared to truncated protein expression is shown on the right resulting from qualitative analysis of the two protein bands in the red circle. The upper band shows the full length protein and the lower band the truncated one.

In order to obtain as much as possible amounts of $\mathrm{NH}_{2} \mathrm{Y}_{730}-\alpha 2$ and $\mathrm{NH}_{2} \mathrm{Y}_{731}-\alpha 2$ for EPR measurements five expressions were run for each $\alpha 2$-mutant. The cell amounts obtained for $8 \mathrm{~L}$ media are summarized below for all expressions together with the induction parameters (Table 2-1).

\begin{tabular}{|c|c|c|c|}
\hline $\begin{array}{c}\text { Expression } \\
\text { of } \mathrm{NH}_{2} \mathbf{Y}_{\mathbf{7 3 0}} \mathbf{\alpha}\end{array}$ & $\begin{array}{c}\mathbf{O D}_{\mathbf{6 0 0}} \text { before induction } \\
\text { with IPTG }\end{array}$ & $\begin{array}{c}\text { Induction after } \mathbf{x} \mathbf{h} \text { at } \\
\mathbf{3 7}{ }^{\circ} \mathbf{C}\end{array}$ & Cell amount [g] \\
\hline 1 & 0.613 & 14.5 & 11.6 \\
\hline 2 & 0.631 & 9.5 & 10.3 \\
\hline 3 & 0.643 & 19.5 & 11.0 \\
\hline 4 & 0.654 & 14.5 & 11.1 \\
\hline 5 & 0.664 & 16 & 13.1 \\
\hline
\end{tabular}

\begin{tabular}{|c|c|c|c|}
\hline $\begin{array}{c}\text { Expression } \\
\text { of } \mathrm{NH}_{2} \mathrm{Y}_{731}-\alpha\end{array}$ & $\begin{array}{c}\mathrm{OD}_{600} \text { before induction } \\
\text { with IPTG }\end{array}$ & $\begin{array}{c}\text { Induction after } \mathbf{x} \mathrm{h} \text { at } \\
\mathbf{3 7}{ }^{\circ} \mathbf{C}\end{array}$ & Cell amount [g] \\
\hline 1 & 0.633 & 17.5 & 13.6 \\
\hline
\end{tabular}




\begin{tabular}{|c|c|c|c|}
\hline 2 & 0.638 & 9.5 & 11.3 \\
\hline 3 & 0.656 & 19.5 & 11.0 \\
\hline 4 & 0.660 & 14.5 & 12.1 \\
\hline 5 & 0.664 & 16 & 11.1 \\
\hline
\end{tabular}

Table 2-1. Summarized cell amounts and induction parameters for ten expressions of $\mathrm{NH}_{2} \mathrm{Y}_{730 / 731^{-}}-\alpha$. Table above showing the expression of $\mathrm{NH}_{2} \mathrm{Y}_{730}$ and lower table the expression of $\mathrm{NH}_{2} \mathrm{Y}_{731}-\alpha$, respectively. The reason for different induction time points are not clear and can not be correlated with the amount of obtained cells. $\mathrm{OD}_{600}$ is the measured optical density at $600 \mathrm{~nm}$.

\subsection{Discussion}

Overall the exemplified expression and purification procedure was successful in case of wt $\alpha 2$. The suppressor tRNA/aminoacyl-tRNA synthetase methodology could be applied but lead to minor yields of $\mathrm{NH}_{2} \mathrm{Y}_{730 / 731}-\alpha 2$ due to expression of truncated $\alpha 2$ proteins. Although one purification step was skipped the obtained protein yields were half of the protein yields for wt $\alpha 2$. Therefore attempts to increase $\mathrm{NH}_{2} \mathrm{Y}$ concentrations in order to obtain greater expressions yields of $\mathrm{NH}_{2} \mathrm{Y}_{730 / 731-}-\alpha 2$ have been performed, hereby going in some expressions to $5 \mathrm{mM} \mathrm{NH}_{2} \mathrm{Y}$. However, that attempt lead to no significant increase of the amount of full length $\mathrm{NH}_{2} \mathrm{Y}_{730 / 731}-\alpha 2$ with respect to their truncated forms. It was concluded that the increased unnatural amino acid concentration was not the minimizing factor during the expression rather other factors which deal with the expression system itself.

Using the in vivo methodology leads to $100-150 \mathrm{mg}$ quantities of each $\mathrm{NH}_{2} \mathrm{Y}-\alpha 2$ and shows that unnatural amino acid incorporation of $\mathrm{NH}_{2} \mathrm{Y}$ in proteins of nearly $100 \mathrm{kDa}$ size is feasible. With respect to E.coli RNR the methodology has been also used to incorporate $\mathrm{NH}_{2} \mathrm{Y}$ at position 413 in the $\alpha$-subunit in order to examine the pathway dependence of radical propagation and very recently the incorporation at position 356 in the $\beta$-subunit was successful $(8,15)$. Analytical methods like ESI or MALDI TOF are less sensitive in such large sized proteins like $\alpha 2$ (172 kDa) in order to detect for $\mathrm{NH}_{2} \mathrm{Y}$ incorporation. However substitution of $\left[{ }^{14} \mathrm{~N}\right]-\mathrm{NH}_{2} \mathrm{Y}_{730}$ through $\left[{ }^{15} \mathrm{~N}\right]-\mathrm{NH}_{2} \mathrm{Y}_{730}$ changed the EPR spectrum measured in $\mathrm{D}_{2} \mathrm{O}$ buffer drastically due to the different spin states of nitrogen nuclei $\left(\mathrm{I}=1\right.$ for ${ }^{14} \mathrm{~N}$ and 
$I=1 / 2$ for $\left.{ }^{15} \mathrm{~N}\right)$. The incorporation could therefore be directly visualized in the EPR spectrum (16). Incubation of $\mathrm{NH}_{2} \mathrm{Y}_{730 / 731}-\alpha 2$ with $\beta 2$, substrate and allosteric effector results in the formation of an $\mathrm{NH}_{2} \mathrm{Y}$ radical $\left(\mathrm{NH}_{2} \mathrm{Y}^{\circ}\right)$ (Figure 2-8) which will be the topic of the next chapter where the new protein radical was examined by multifrequency EPR spectroscopy at position 730 .
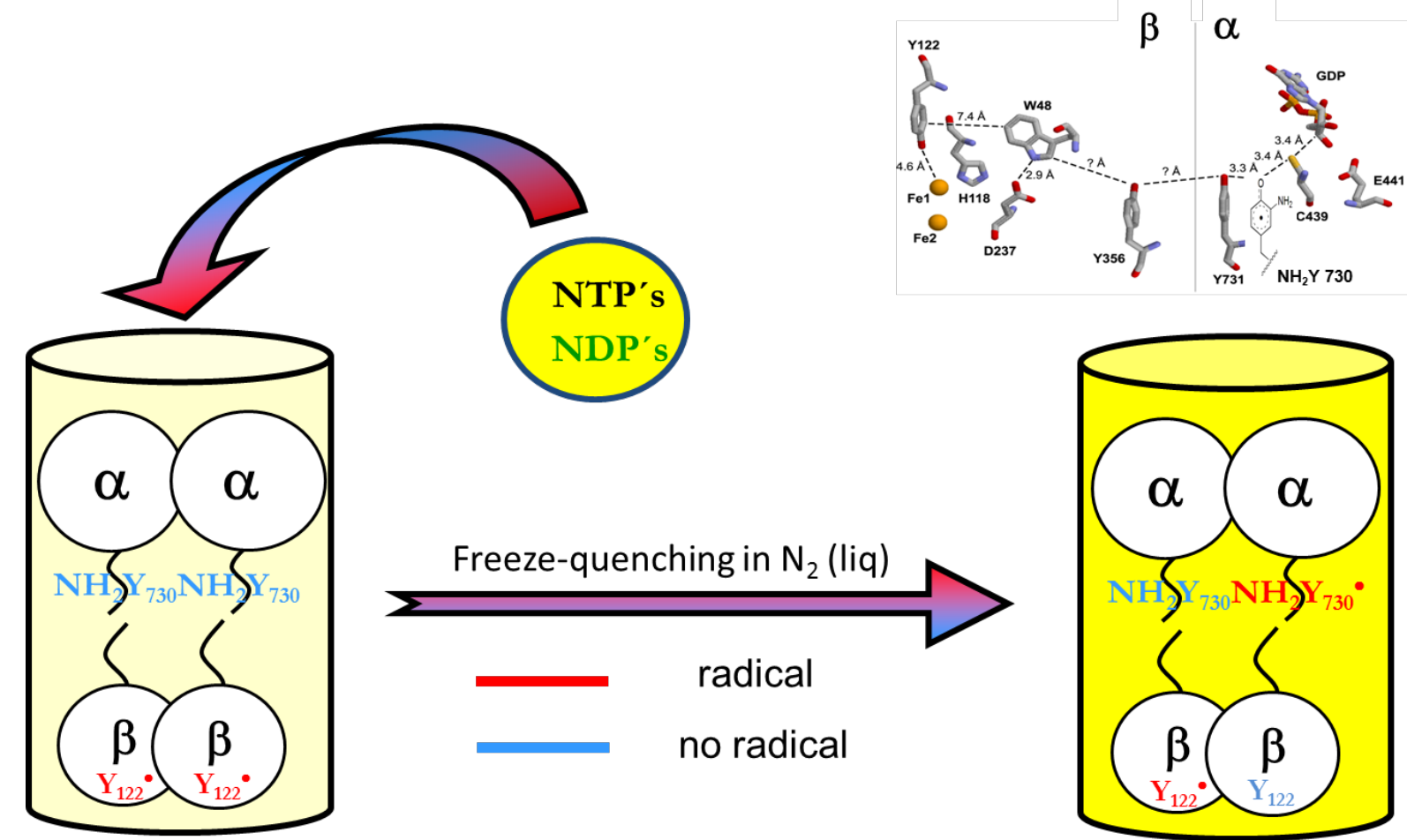

Figure 2-8. Incubation of the $\mathrm{NH}_{2} \mathrm{Y}_{730}-\alpha 2: \beta 2$ complex with NDP's and NTP's. The new protein radical has been formed after quenching the reaction in the second time scale. Quite interestingly the complex shows nucleotide reduction activity which is $4 \%$ that of wt $\alpha 2$ in case of $\mathrm{NH}_{2} \mathrm{Y}_{730}-\alpha 2$. Right inset: The proposed radical propagation pathway in E.coli RNR.

\subsection{Materials and Methods}

\section{Materials}

Petri dish plates, Erlenmeyer flasks, shaker/incubator, centrifuges, French press, gel plates, polypropylene tubes, Falcon tubes, BactoAgar, 3-aminotyrosine $\left(\mathrm{NH}_{2} \mathrm{Y}\right)$, tetracycline (Tet), ampicillin (Amp), L-leucine (Leu), D-biotin, thiamine $\mathrm{HCl}$, NADPH, ATP, CDP, ethylenediamine 
tetraacetic acid (EDTA), Tris- $\mathrm{HCl}$, Tris-Base (Tris = Tris(hydroxymethyl)aminomethane), HEPES ((4-(2-Hydroxyethyl)piperazine-1-ethanesulfonic acid), glycerol, Bradford Reagent for Bradford assay, Sephadex G-25, phenylmethanesulfonylfluoride (PMSF), streptomycin sulfate, Isopropyl- $\beta$-D-thiogalactopyranoside (IPTG), DL-dithiothreitol (DTT), E.coli DH10 $\beta$ competent cells, $\mathrm{MoNa}_{2} \mathrm{O}_{4} \cdot 2 \mathrm{H}_{2} \mathrm{O}, \mathrm{CoCl}_{2}, \mathrm{CuSO}_{4} \cdot 5 \mathrm{H}_{2} \mathrm{O}, \mathrm{MnSO}_{4} \cdot \mathrm{H}_{2} \mathrm{O}, \mathrm{MgSO}_{4} \cdot 7 \mathrm{H}_{2} \mathrm{O}, \mathrm{ZnSO}_{4} \cdot 7 \mathrm{H}_{2} \mathrm{O}$, $\mathrm{FeCl}_{2} \cdot 4 \mathrm{H}_{2} \mathrm{O}, \mathrm{CaCl}_{2} \cdot 2 \mathrm{H}_{2} \mathrm{O}, \mathrm{H}_{3} \mathrm{BO}_{3}$ and $1 \mathrm{M} \mathrm{HCl}$, dd $\mathrm{H}_{2} \mathrm{O}, \beta$-mercaptoethanol, Laemmli buffer, sodiumdodecyl sulphate (SDS), acrylamide, bis-acrylamide, ammonium persulfate (APS), $\mathrm{N}, \mathrm{N}, \mathrm{N}$ ', N'-tetramethylethylenediamine (TEMED), D-biotin, thiamine.

Media and solutions used for the expression and purification of wt $\alpha 2$ and $\mathrm{NH}_{2} \mathrm{Y}-\alpha 2 \mathrm{~s}$ :

1) LB medium

2) 2YT medium

3) SOC medium

4) ampicillin stock solution $(100 \mathrm{mg} / \mathrm{mL})$

5) tetracycline stock solution $(25 \mathrm{mg} / \mathrm{mL})$

6) $1000 x$ heavy metal stock solution (M9) contains the following per L: $500 \mathrm{mg}$ $\mathrm{MoNa}_{2} \mathrm{O}_{4} \cdot 2 \mathrm{H}_{2} \mathrm{O}, 250 \mathrm{mg} \mathrm{CoCl}, 175 \mathrm{mg} \mathrm{CuSO}{ }_{4} \cdot 5 \mathrm{H}_{2} \mathrm{O}, 1 \mathrm{~g} \mathrm{MnSO}_{4} \cdot \mathrm{H}_{2} \mathrm{O}, 8.75 \mathrm{~g}$ $\mathrm{MgSO}_{4} \cdot 7 \mathrm{H}_{2} \mathrm{O}, 1.25 \mathrm{~g} \mathrm{ZnSO}_{4} \cdot 7 \mathrm{H}_{2} \mathrm{O}, 1.25 \mathrm{~g} \mathrm{FeCl}_{2} \cdot 4 \mathrm{H}_{2} \mathrm{O}, 2.5 \mathrm{~g} \mathrm{CaCl}_{2}{ }^{\circ} 2 \mathrm{H}_{2} \mathrm{O}, 1 \mathrm{~g} \mathrm{H}_{3} \mathrm{BO}_{3}$ and $1 \mathrm{M} \mathrm{HCl}$

7) Glycerol M9 minimal media leucine (GMML) contains final concentrations of: 1\% (v/v) glycerol, $1 \times \mathrm{M} 9$ salts, $0.05 \%(\mathrm{w} / \mathrm{v}) \mathrm{NaCl}$, D-biotin $(1 \mu \mathrm{g} / \mathrm{mL})$, thiamine $(1 \mu \mathrm{g} / \mathrm{mL}), 1 \mathrm{mM} \mathrm{MgSO}_{4}, 0.1 \mathrm{mM} \mathrm{CaCl}_{2}$, and $0.3 \mathrm{mM} \mathrm{L-leucine}$

8) $1 \mathrm{~mL}$ Laemmli buffer solution consists of $30 \mu \mathrm{L} \beta$-mercaptoethanol

9) $\alpha 2$ buffer consist of $50 \mathrm{mM}$ Tris-HCl, $1 \mathrm{mM}$ EDTA, 5\% (v/v) glycerol with the $\mathrm{pH}$ adjusted to $\mathrm{pH} 7.6$

10) RNR assay buffer consists of $50 \mathrm{mM}$ Hepes, $15 \mathrm{mM} \mathrm{MgSO}_{4}, 1 \mathrm{mM}$ EDTA with the pH adjusted to 7.6

11) stacking buffer contains $0.5 \mathrm{M}$ TrisBase with the $\mathrm{pH}$ adjusted to 6.8

12) staining and destaining solutions from BioRad

The concentrations of $\alpha 2, \mathrm{NH}_{2} \mathrm{Y}_{730}-\alpha 2$ and $\mathrm{NH}_{2} \mathrm{Y}_{731}-\alpha 2$ were determined in RNR assay buffer by UV/Vis spectroscopy using the Lambert Beer law $\left(\varepsilon_{280 \mathrm{~nm}}=189 \mathrm{mM}^{-1} \mathrm{~cm}^{-1}\right)$. 


\section{Preparation of LB plates for the transformation procedure}

$1.5 \mathrm{~g}$ BactoAgar and 2 to $2.5 \mathrm{~g}$ LB-medium have been dissolved in $100 \mathrm{~mL} \mathrm{ddH_{2 }} \mathrm{O}$.

For one plate $25 \mathrm{~mL}$ LB-agar-media has to be considered and autoclaved. After cooling down antibiotics have been put with the appropriate concentrations to the LB-agar-media at a temperature around $50{ }^{\circ} \mathrm{C}$ and afterwards the media was plated on a Petri dish plate where it cooled further down.

\section{Transformation of pTrc-nrdA $730 / 731$ TAG and pAC- $\mathrm{NH}_{2} \mathrm{Y}-\mathrm{RS}$ into $\mathrm{DH} 10 \beta$ cells}

Competent $\mathrm{DH} 10 \beta$ cells were thawed on wet ice and gently mixed before putting an aliquot of $100 \mathrm{~mL}$ into a pre-cooled polypropylene tube. The unused cells were then refrozen in dry ice for 5 minutes before freezing them further with liquid $N_{2} .1 \mathrm{~mL}$ of plasmid solution (containing pTrc-nrdA ${ }_{730}$ TAG or pTrc-nrdA ${ }_{731}$ TAG and pAC- $\mathrm{NH}_{2} \mathrm{Y}-\mathrm{RS}$ ) was added to $100 \mathrm{~mL}$ competent cell solution. The cells were incubated together with the plasmids for 30 minutes and heat-shocked for $45 \mathrm{sec}$ in a $42{ }^{\circ} \mathrm{C}$ water bath. The polypropylene tubes should not be shaken during this period while the plasmids are penetrating into the cell. Afterwards the cells have been placed for 2 minutes on ice and $900 \mu \mathrm{L}$ of SOC-medium was added to the polypropylene tubes at RT and incubated for $1 \mathrm{~h}$ at $37{ }^{\circ} \mathrm{C}$ and $225 \mathrm{rpm} .100 \mathrm{~mL}$ of SOC-medium was spread on LB-plates with $100 \mathrm{mg} / \mathrm{mL}$ ampicillin and incubated for two days at $37{ }^{\circ} \mathrm{C}$. The grown colonies have been put back then into the fridge at $4{ }^{\circ} \mathrm{C}$.

\section{Column preparation for the purification of $\mathrm{NH}_{2} \mathrm{Y}_{730}-\alpha 2$ and $\mathrm{NH}_{2} \mathrm{Y}_{731}-\alpha 2$}

The G-25 column was prepared through washing with $2 \mathrm{CV}$ of $0.2 \mathrm{M} \mathrm{NaOH}, 10 \mathrm{CV}$ of $\mathrm{ddH}_{2} \mathrm{O}$ and $5 \mathrm{CV}$ of $\alpha 2$-buffer. The DEAE (diethylaminoethyl) column was washed with $5 \mathrm{CV}$ of $1 \mathrm{M}$ $\mathrm{NaCl}, 10 \mathrm{CV}$ of $\mathrm{ddH}_{2} \mathrm{O}$ and $10 \mathrm{CV}$ of $\alpha 2$-buffer and the dATP column was washed with $2 \mathrm{CV}$ of $0.1 \%$ SDS solution, $10 \mathrm{CV}$ of $\mathrm{dd}_{2} \mathrm{O}$ and $5 \mathrm{CV}$ of $\alpha 2$-buffer. The three mentioned columns were already packed whereas a G-25 column $(1.5 \mathrm{~cm} \times 25 \mathrm{~cm}, 45 \mathrm{~mL})$ has been prepared for the removal of small molecules like DTT or ATP. The packing of the small G-25 column was performed by using $20 \mathrm{~g}$ of Sephadex G-25 and suspending it in $100 \mathrm{~mL} \mathrm{dd}_{2} \mathrm{O}$. The suspension was temporarily stirred in a beaker for $3 \mathrm{~h}$ at RT and afterwards the column was filled with the resin and $1 \mathrm{~L}$ of $\mathrm{ddH}_{2} \mathrm{O}$ has been passed through. 
Preparation of SDS-gels for the analysis of purification and expression steps of $\mathrm{NH}_{2} \mathrm{Y}_{730}-\alpha 2$ and $\mathrm{NH}_{2} \mathrm{Y}_{731}-\alpha 2$

The $8 \%$ resolving gel was prepared by adding $4.7 \mathrm{~mL} \mathrm{ddH_{2 }} \mathrm{O}$ to a $15 \mathrm{~mL}$ Falcon together with $2.7 \mathrm{~mL} 30 \%$ Acrylamide/Bis, $2.5 \mathrm{~mL}$ stacking buffer and $0.1 \mathrm{~mL}$ SDS solution (10 \% (w/v)). The Falcon was shaken gently and afterwards $50 \mu \mathrm{L}$ of APS solution and $10 \mu \mathrm{L}$ of TEMED have been added before pouring the gel over $3 / 4$ of the plate area. After the resolving gel got stiff the $4 \%$ stacking gel was prepared by adding $6.1 \mathrm{~mL} \mathrm{ddH_{2 }} \mathrm{O}$ to a $15 \mathrm{~mL}$ Falcon tube together with $1.3 \mathrm{~mL} 30 \%$ Acrylamide/Bis solution. The volume of the stacking buffer and SDS solution $(10 \%(\mathrm{w} / \mathrm{v}))$ was the same. The Falcon was shaken gently and afterwards $50 \mu \mathrm{L}$ of APS solution and $10 \mu \mathrm{L}$ of TEMED have been added until the rest of the gel plate has been moistened. $3 / 4$ of the gel plate consisted the $8 \%$ resolving gel which is needed for the separation of different protein weights. The $4 \%$ stacking gel contained the units for sample loading (maximally 9-10 units). Usually gels were run with a voltage of $220 \mathrm{~V}$ for 40 minutes and were stained and destained with solutions from BioRad.

\section{Expression of $\mathrm{NH}_{2} \mathrm{Y}_{730}-\alpha 2$ and $\mathrm{NH}_{2} \mathrm{Y}_{731}-\alpha 2$ subunit}

The pTrc-nrdA $A_{730 / 731}$ TAG/pAC-NH $\mathrm{NH}_{2} \mathrm{Y}-\mathrm{RS}$ expression system was used to express $\mathrm{NH}_{2} \mathrm{Y}_{730 / 731}-\alpha 2$. E. coli $\mathrm{DH} 10 \beta$ cells were transformed with vectors $\mathrm{pTrc}-\mathrm{nrdA} \mathrm{A}_{730 / 731}$ TAG and pAC- $\mathrm{NH}_{2} \mathrm{Y}$-RS and the cells were grown at $37{ }^{\circ} \mathrm{C}$ on LB/Agar plates containing Amp (100 $\mu \mathrm{g} / \mathrm{mL})$ for 2 days. All liquid cell culture growths contained Amp $(100 \mu \mathrm{g} / \mathrm{mL})$ and Tet $(25$ $\mu \mathrm{g} / \mathrm{mL}$ ) and were carried out in a shaker/incubator at $37{ }^{\circ} \mathrm{C}$ and $200 \mathrm{rpm}$. A single colony from the LB/Agar plate was inoculated into $7 \mathrm{~mL}$ of $2 \mathrm{YT}$ medium. The whole volume of the saturated culture was then transferred after 2 days to $250 \mathrm{~mL}$ of $2 \mathrm{YT}$ medium and grown to saturation for 20 h. $25 \mathrm{~mL}$ of this culture were then diluted into each of $8 \times 6$ L Erlenmeyer flasks, each containing $1 \mathrm{~L}$ of glycerol-M9-medium. The $\mathrm{OD}_{600}$ of 0.6 was reached after $14-16$ $\mathrm{h}$ of incubation. Immediately DTT and $\mathrm{NH}_{2} \mathrm{Y}$ were added to a final concentration of $0.1 \mathrm{mM}$ and $1 \mathrm{mM}$ (in some cases the concentration was varied up to $5 \mathrm{mM}$ ). After $15 \mathrm{~min}$, IPTG was then added to a final concentration of $1 \mathrm{mM}$ for induction and the growth continued for 4.5 h. The cells were then centrifuged, frozen in liquid $\mathrm{N}_{2}$ and stored at $-80^{\circ} \mathrm{C}$. $1.5 \mathrm{~g}$ of cell paste per $L$ cell culture was usually obtained after the expression. Expression of $\mathrm{NH}_{2} \mathrm{Y}_{731}-\alpha 2$ was carried out in an identical fashion. The introduction to the expression procedure of 
mutant $\alpha 2 \mathrm{~s}$ was performed by Clement Chan. wt $\alpha 2$ was expressed in a similar way and was used primarily to test the purification procedure for $\mathrm{NH}_{2} \mathrm{Y}_{730 / 731}-\alpha 2$.

\section{Purification of wt $\alpha 2, \mathrm{NH}_{2} \mathrm{Y}_{730}-\alpha 2$ and $\mathrm{NH}_{2} \mathrm{Y}_{731}-\alpha 2$ subunit}

All purification steps were performed at $4{ }^{\circ} \mathrm{C}$. $\mathrm{NH}_{2} \mathrm{Y}_{730 / 731}-\alpha 2$ was typically purified from $10 \mathrm{~g}$ of wet cell paste. Each g of cell paste was resuspended in $5 \mathrm{~mL}$ of $\alpha 2$ buffer $(\mathrm{pH} 7.6)$. Additionally the solution was supplemented with PMSF $(0.5 \mathrm{mM})$ and DTT $(4 \mathrm{mM})$. The whole French press equipment was cooled for at least 30 min on ice. Usually $35 \mathrm{~mL}$ of cell suspension were lysed by double passage through a French pressure cell operating at 14000 psi. After removal of cell debris by centrifugation (24000 rpm, $20 \mathrm{~min}, 4{ }^{\circ} \mathrm{C}$ ), DNA was precipitated by dropwise addition of 0.2 volumes of $\alpha 2$ buffer containing streptomycin sulphate $(8 \% \mathrm{w} / \mathrm{v})$. The suspension was stirred for an additional $30 \mathrm{~min}$, and the precipitated DNA was removed by centrifugation (24000 rpm, $20 \mathrm{~min}, 4^{\circ} \mathrm{C}$ ). Afterwards $4 \mathrm{~g}$ of solid $\left(\mathrm{NH}_{4}\right)_{2} \mathrm{SO}_{4}$ was added per $10 \mathrm{~mL}$ of supernatant over $20 \mathrm{~min}$ (66\% saturation). The suspension was stirred for an additional $30 \mathrm{~min}$ and the precipitated protein was isolated by centrifugation $\left(24000 \mathrm{rpm}, 20 \mathrm{~min}, 4^{\circ} \mathrm{C}\right.$ ). The pellet was redissolved in a minimal volume of $\alpha 2$ buffer and desalted using a pre-equilibrated Sephadex G-25 column $(1.5 \mathrm{~cm} \times 25 \mathrm{~cm}, 45$ $\mathrm{mL}$ ). The desalted protein was loaded at a flow rate of $0.5 \mathrm{~mL} / \mathrm{min}$ directly onto a dATP column $(1.5 \mathrm{~cm} \times 4 \mathrm{~cm}, 6 \mathrm{~mL})$, which had been equilibrated in $\alpha 2$ buffer. The column was washed with $10 \mathrm{CV}$ of $\alpha 2$ buffer. $\mathrm{NH}_{2} \mathrm{Y}_{730 / 731^{-}} \alpha 2$ was then eluted in 3-4 CV of $\alpha 2$ buffer containing also ATP $(10 \mathrm{mM})$ and DTT $(10 \mathrm{mM})$. The eluted fractions were quickly judged by the Bradford test. ATP and DTT were then subsequently removed by Sephadex G-25 chromatography. Optionally a pre-equilibrated DEAE sepharose column can be used before going over to the dATP column with a $1 \times 1$ gradient of $\alpha 2$ to $\alpha 2$ containing $400 \mathrm{mM} \mathrm{NaCl}$. This step has been used for the purification of wt $\alpha 2$ as the expression yields were much higher (3.4 g of cell paste per $L$ cell culture instead of $1.3 \mathrm{~g}$ ). However in case of $\mathrm{Y}_{730 / 731} \mathrm{NH}_{2} \mathrm{Y}$ $\alpha 2$ it was decided to skip this step in order to increase final protein yields. All purified proteins were flash-frozen in small aliquots in liquid $\mathrm{N}_{2}$ and stored at $-80^{\circ} \mathrm{C}$. Typically $2-3 \mathrm{mg}$ of purified $\mathrm{NH}_{2} \mathrm{Y}_{730 / 731}-\alpha 2$ was obtained per $\mathrm{g}$ of wet cell paste and $5 \mathrm{mg}$ of wt $\alpha 2$. 
Chapter 3

Multifrequency EPR characterization of 3-aminotyrosyl radicals on the radical propagation pathway of E.coli ribonucleotide reductase 


\subsection{Introduction}

To investigate the roles of Ys in the radical propagation of E.coli RNR the in vivo suppressor tRNA/RS method was used in order to replace site-specifically each residue with 3-aminotyrosine $\left(\mathrm{NH}_{2} \mathrm{Y}\right)$. After incubation of $\mathrm{NH}_{2} \mathrm{Y}$ - $\alpha 2 \mathrm{~s}$ with substrate, effector and $\beta 2$ the $\mathrm{Y}_{122}{ }^{\circ}$ signal gets diminished and concomitant a new radical species is detected. Based on UV-vis, $\mathrm{X}$-band EPR and PELDOR spectroscopy this species was categorized to be a $\mathrm{NH}_{2} \mathrm{Y}^{\bullet}$ $(1,2)$. The same occurs when $\mathrm{NH}_{2} \mathrm{Y}-\beta 2$ is incubated with substrate, effector and $\alpha 2(3)$. However a detailed EPR spectroscopic investigation has not been performed yet which is the key in order to use $\mathrm{NH}_{2} \mathrm{Y}$ as a probe for PCET reactions in general and the radical propagation step specifically in E.coli RNR.

In this chapter the electronic and molecular structure of $\mathrm{NH}_{2} \mathrm{Y}_{730}{ }^{\circ}$ will be examined in detail which is an essential first step in using the structure of $\mathrm{NH}_{2} \mathrm{Y}_{730}{ }^{\circ}$ to formulate a model for a PCET mechanism within $\alpha 2$ and to use the obtained EPR parameter set further for the characterization of $\mathrm{NH}_{2} \mathrm{Y}_{731}{ }^{\bullet}$ in $\alpha 2$ and $\mathrm{NH}_{2} \mathrm{Y}_{356}{ }^{\circ}$ in $\beta 2$. The analysis of the external hydrogenbond network together with the PCET mechanism at position 730 will be documented in Chapter 4 through high-field ENDOR spectroscopy in conjunction with DFT calculations. Furthermore it is anticipated that $\mathrm{NH}_{2} \mathrm{Y}$ can function in general in other systems as a reporter for PCET mechanisms where transient $\mathrm{Y}^{\circ} \mathrm{s}$ participate in catalysis.

Multifrequency pulsed EPR spectroscopy experiments in different buffer media $\left(D_{2} O\right.$ and $\mathrm{H}_{2} \mathrm{O}$ buffer) from 9 to $180 \mathrm{GHz}$ were used in order to extract systematically each EPR parameter of choice. For example as mentioned in Chapter 1 the g-values can be extracted with highest accuracy at the highest frequency (here $180 \mathrm{GHz}$ ) and the determination of hyperfine parameters of different proton nuclei which substantially contribute to the shape of EPR spectra is performed through the simulation of $1^{\text {st }}$ derivative $94 \mathrm{GHz}$ and $9 \mathrm{GHz} \mathrm{EPR}$ spectra in $\mathrm{D}_{2} \mathrm{O}$ and $\mathrm{H}_{2} \mathrm{O}$ buffer. The extensive simulation procedure is documented in the

results section of this chapter. Below the first pulsed EPR spectra on the $\alpha-\mathrm{NH}_{2} \mathrm{Y}_{730}$ are shown at 9, 94 and $180 \mathrm{GHz}$ (Figure 3-1), respectively. 


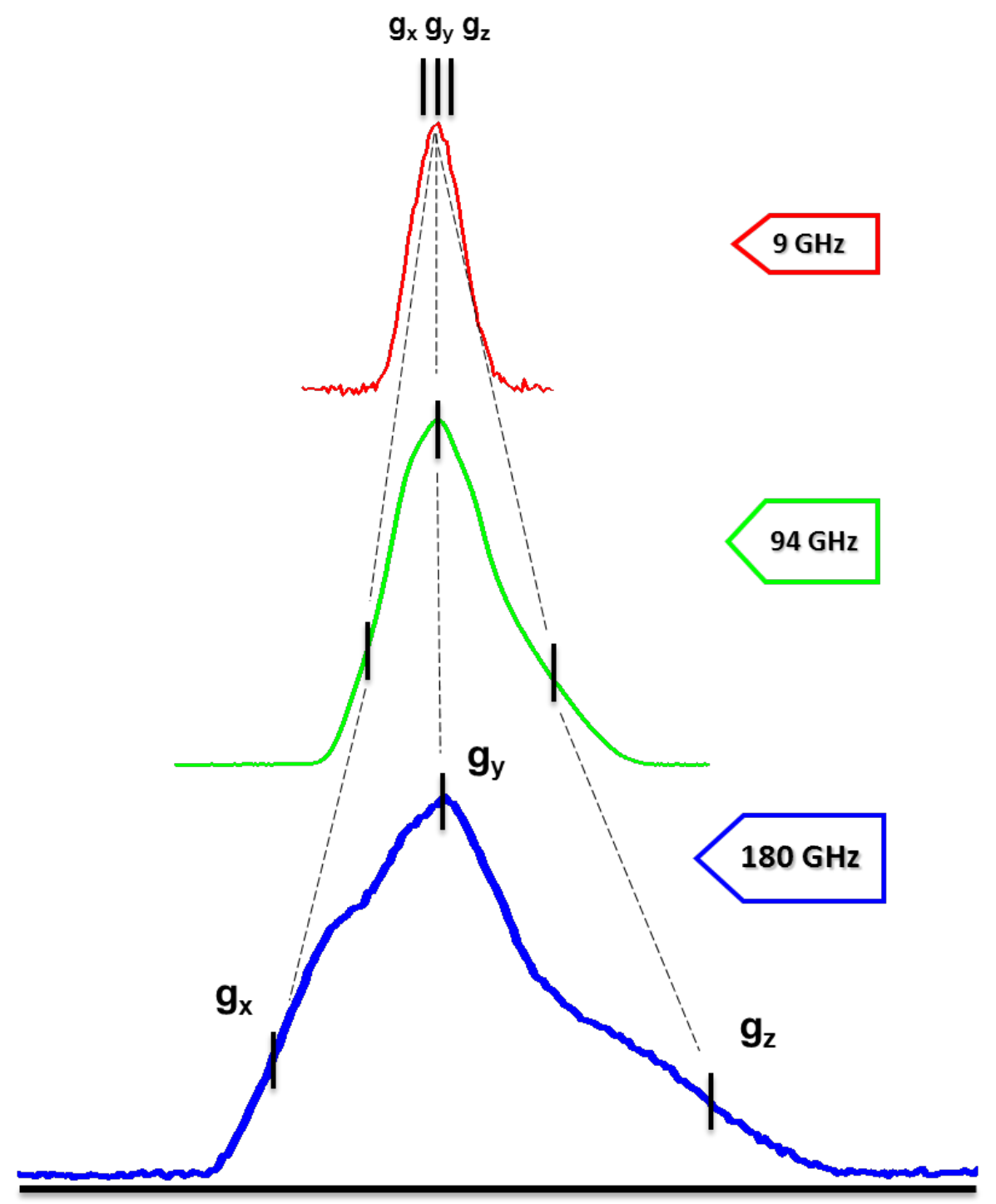

Field (G)

Figure 3-1. Multifrequency EPR spectra of $\alpha-\mathrm{NH}_{2} \mathrm{Y}_{730^{\circ}}$ in the presence of CDP/ATP as a substrate/effector pair in $\mathrm{H}_{2} \mathrm{O}$ assay buffer. The increase in g-resolution with increasing frequency and higher fields is demonstrated. The spectrum at $180 \mathrm{GHz}$ yields g-values of $\mathrm{g}_{\mathrm{x}}=2.0052, \mathrm{~g}_{\mathrm{y}}=2.0042$ and $g_{z}=2.0022$. Although the g-values can be discriminated in the $94 \mathrm{GHz}$ spectrum they have been extracted with highest accuracy at $180 \mathrm{GHz}$ (the error here is \pm 0.0001 ).

Until now no EPR studies were performed on proteins containing 3-aminotyrosyl radicals and even EPR studies on the amino acid radical itself are missing. However plenty of studies exist on the $o$-aminophenol radical (4-9) and on $Y^{*}$ s for many different proteins $(10,11)$. $9 \mathrm{GHz}$ EPR studies on the o-aminophenol radical reveal that the two major coupling contributions arise from the nitrogen and the hydrogens of the amino group. Depending on 
the solvent the isotropic nitrogen hyperfine coupling constant varies between 12.4 and 13.3 $\mathrm{MHz}$. The isotropic hyperfine coupling constants for the two amino protons are larger (up to $21 \mathrm{MHz}$ ) and are reported to have different values especially in studies which are performed in $\mathrm{CH}_{2} \mathrm{Cl}_{2} /$ THF (THF = tetrahydrofuran) which is a good mimic for a protein environment. $9 \mathrm{GHz}$ studies in water revealed also a $\mathrm{g}_{\text {iso }}$ of 2.0037 which comes close to our $\mathrm{g}_{\text {iso }}$ of 2.0038 of the $\alpha-\mathrm{NH}_{2} \mathrm{Y}_{730}$ indicating a hydrogen-bond environment around the radical.

As it was shown in the introduction for $\mathrm{Ys}^{\circ}$, a substantial shift to 2.0076 of the $g_{\mathrm{x}}$ value indicates already the possibility of an $\mathrm{H}$-bond. Our $\mathrm{g}_{\mathrm{x}}$-value is much lower (2.0052) and one reason for this is the possibility of an intramolecular $\mathrm{H}$-bond between $\mathrm{N}$ and $\mathrm{O}$ of the radical species as reported through early INDO calculations by Loth et al. (12). A more recent computational study on the hybridization state of the nitrogen in anilines showed also that pyramidalization can occur giving a geometrical structure which can be explained through a partially sp3-hybridized state (13).

These different spectroscopical and theoretical studies provided the foundation to understand our radical system. However not all structural and electronic parameters could be obtained through the before mentioned studies (i.e. the hyperfine and g-tensor) which are necessary for a detailed characterization of the 3-aminotyrosyl radical in $\alpha 2$ and $\beta 2$. We performed therefore a systematic analysis of the EPR spectra at frequencies from 9 - 180 $\mathrm{GHz}$ in order to obtain a detailed picture about the electronic and molecular structure. Our data give insight into the g-values, the orientation of the $\beta$-protons, the hybridization state of the amino nitrogen and the orientation of the amino protons relative to the plane of the aromatic ring (Figure 3-2). The hyperfine couplings from which we can extract the geometry of the amino group are consistent hereby with an intramolecular hydrogen bond within $\mathrm{NH}_{2} \mathrm{Y}_{730^{\circ}}$. 


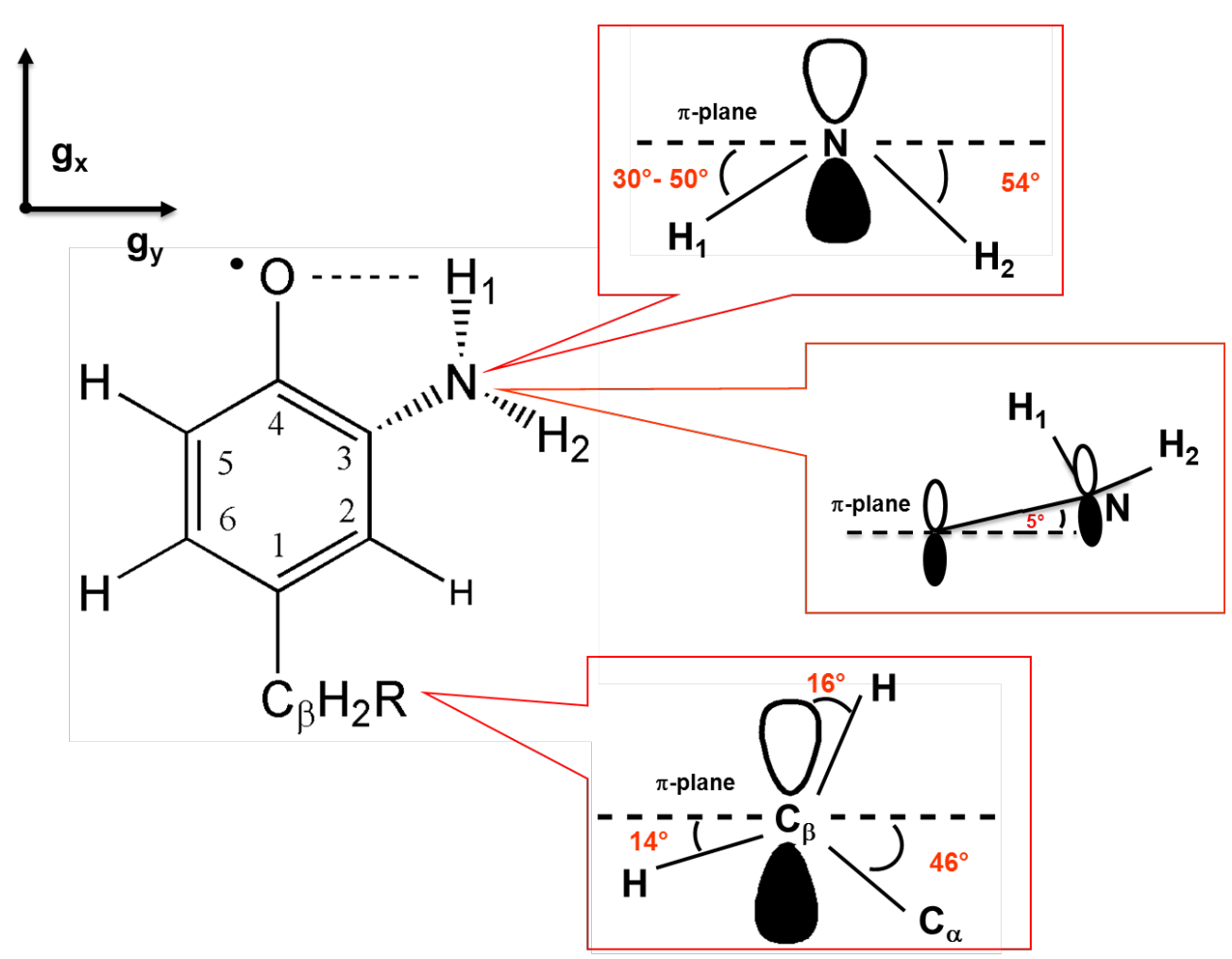

Figure 3-2. Structure of $\mathrm{NH}_{2} \mathrm{Y}_{730}$ ' showing the intramolecular hydrogen-bond represented by dashed lines. The out-of-plane angles for the $\mathrm{NH}_{2}$ group and the dihedral angles for the $\mathrm{C} \beta$-methylene protons with respect to the $\pi$-plane are shown in the red insets.

A sp2 hybridized state of the amino group would result in $\mathrm{C}-\mathrm{N}-\mathrm{H}$ angles which are $120^{\circ}$. Our angles obtained from the EPR studies are $106^{\circ}$ and $105^{\circ}$ respectively and express the sp3 hybridized nature of the amino group. With the calotte model option in PyMOL which represents the electron density around nuclei, we can also observe a small overlap of H1 electron density of the amino group with the oxygen electron density of the C-O group (Figure 3-3). 

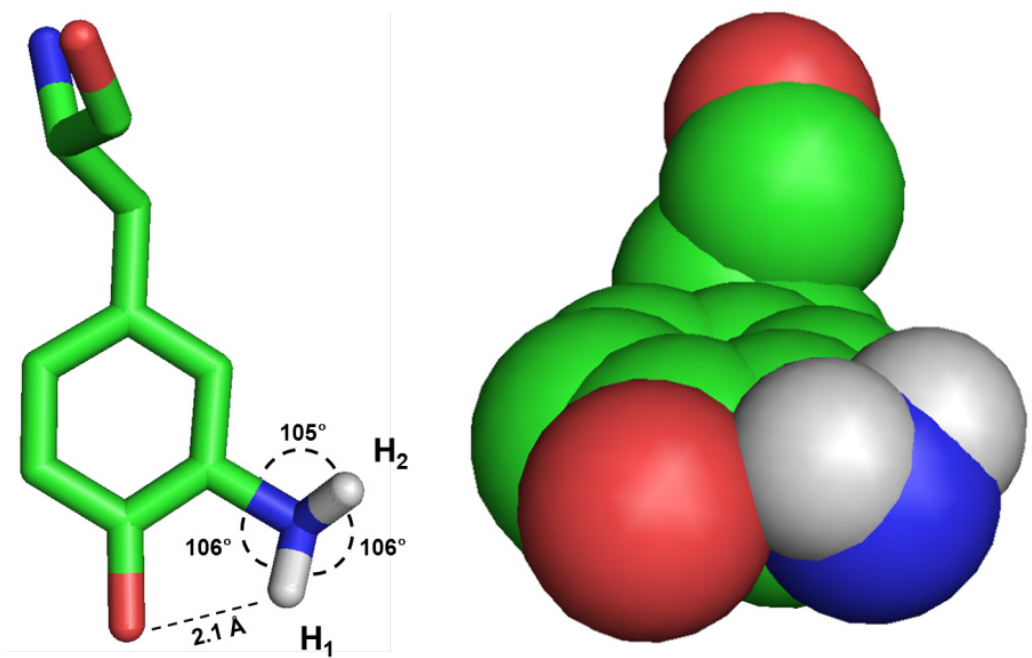

Figure 3-3. PyMol structure of $\mathrm{NH}_{2} \mathrm{Y}_{730^{\circ}}$. View from top showing the $\mathrm{C}-\mathrm{N}-\mathrm{H}$ bond angles which result in a sp3 hybridized state of the amino group (left). A distance of $2.1 \AA$ between $\mathrm{H} 1$ and O positions $\mathrm{H} 1$ into hydrogen-bond distance and leads to an overlap of their electronic orbitals.

The obtained parameter set which was used in the simulation of 9 and $94 \mathrm{GHz}$ EPR spectra of $\mathrm{NH}_{2} \mathrm{Y}_{730}{ }^{\circ}$ in deuterated and protonated buffer was further used as a starting parameter set for the simulation of $\mathrm{NH}_{2} \mathrm{Y}_{731}{ }^{\circ}$ in $\alpha 2$ and $\mathrm{NH}_{2} \mathrm{Y}_{356}{ }^{\circ}$ in $\beta 2$. An optimization of the hyperfine values and the Euler angles had to be performed in order to simulate these EPR spectra. This resulted for example in case of $\mathrm{NH}_{2} \mathrm{Y}_{731}{ }^{\circ}$ in different structural parameters for the $\mathrm{C}_{\beta}$ methylene protons what could be supported by ${ }^{1} \mathrm{H}$ Davies ENDOR spectroscopy at $34 \mathrm{GHz}$. Moreover we could detect in the $\mathrm{NH}_{2} \mathrm{Y}_{731}$ spectrum for two different freeze quench time points an additional radical signal which could originate from a second radical species. Due to an observed conformational flexibility at position 731 in crystal structures of mutant $\alpha 2 \mathrm{~s}$ the signal stems most probably from $\mathrm{NH}_{2} \mathrm{Y}_{731}$ residing in a different conformation. However this conformational flexibility was not observed in crystal structures of the E.coli wt system. Nevertheless from published crystallographic data on $Y_{730}$ and $Y_{731}$ of other different organisms little variability is case of residue 730 and much greater variability in case of residue 731 has been observed $(3,14-16)$.

$\mathrm{NH}_{2} \mathrm{Y}_{356}{ }^{\circ}$ in $\beta 2$ possess only one conformation due to our EPR analysis and could be simulated with slight adjustments of the hyperfine parameter set. The g-values are smaller in comparison to $\mathrm{NH}_{2} \mathrm{Y}_{730}{ }^{\circ}$ and $\mathrm{NH}_{2} \mathrm{Y}_{731}{ }^{\circ}$ given rise to a different electrostatic environment at position 356 in $\beta 2$. 
Most intriguing in the overall analysis of the $\mathrm{NH}_{2} \mathrm{Ys}^{\circ}$ on the radical propagation pathway is that the parameter set for the simulation of the $\mathrm{NH}_{2}$-group is nearly the same for all three spin trap positions and that the $\beta$-methylene proton couplings differ from one position to the other suggesting different conformations of $\mathrm{NH}_{2} \mathrm{Ys}^{\bullet}$ on the pathway.

\subsection{Results}

\section{Decomposition of $\mathrm{Y}_{122}{ }^{\circ}$ and $\alpha-\mathrm{ND}_{2} \mathrm{Y}_{730}{ }^{\circ}$ at $94 \mathrm{GHz}$ - Electron spin echo spectra at}

\section{0 and $10 \mathrm{~K}$}

In order to filter out the EPR signal of any new radical, like the $\mathrm{ND}_{2} \mathrm{Y}_{730^{\circ}}$ all spectra were recorded at $70 \mathrm{~K}$ where the $\mathrm{Y}_{122}{ }^{\circ}$ in $\beta 2$ is not visible during the electron spin echo experiment. At $10 \mathrm{~K}$ the recorded spectrum is a composite of $\mathrm{Y}_{122}{ }^{\circ}$ and $\mathrm{ND}_{2} \mathrm{Y}_{730} 0^{\circ}$. However at $70 \mathrm{~K}$ the EPR signal is associated only with the $\mathrm{ND}_{2} \mathrm{Y}_{730}{ }^{\circ}$ due to the proximity of the $\mathrm{Y}_{122}{ }^{\circ}$ to the di-iron cluster which causes the fast relaxation of the electron species (4.6 $\AA$ distance between Fe1 and the oxygen of the Tyr- $0^{\circ}$ in $\beta 2$ ). The $94 \mathrm{GHz}$ spectra measured at the two different temperatures are displayed in absorptive mode showing that the composite spectrum consisting of $\mathrm{Y}_{122}{ }^{\bullet}$ and $\mathrm{ND}_{2} \mathrm{Y}_{730}{ }^{\bullet}$ exhibits a much broader line width due to the higher $g_{x}$-value of the $Y_{122}{ }^{\circ}\left(g_{x}=2.00912\right.$ for $Y_{122}{ }^{\circ} ; g_{x}=2.0052$ for $\left.N D_{2} Y_{730}{ }^{\circ}\right)$ (Figure 3-4). In general the method for filtering out the $Y_{122}{ }^{\bullet}$ signal has been applied throughout all EPR experiments concerning the $\mathrm{ND}_{2} \mathrm{Y}^{\bullet}$ at different positions of the radical propagation pathway ( $\alpha-730, \alpha-731$ and $\beta-356)$. 


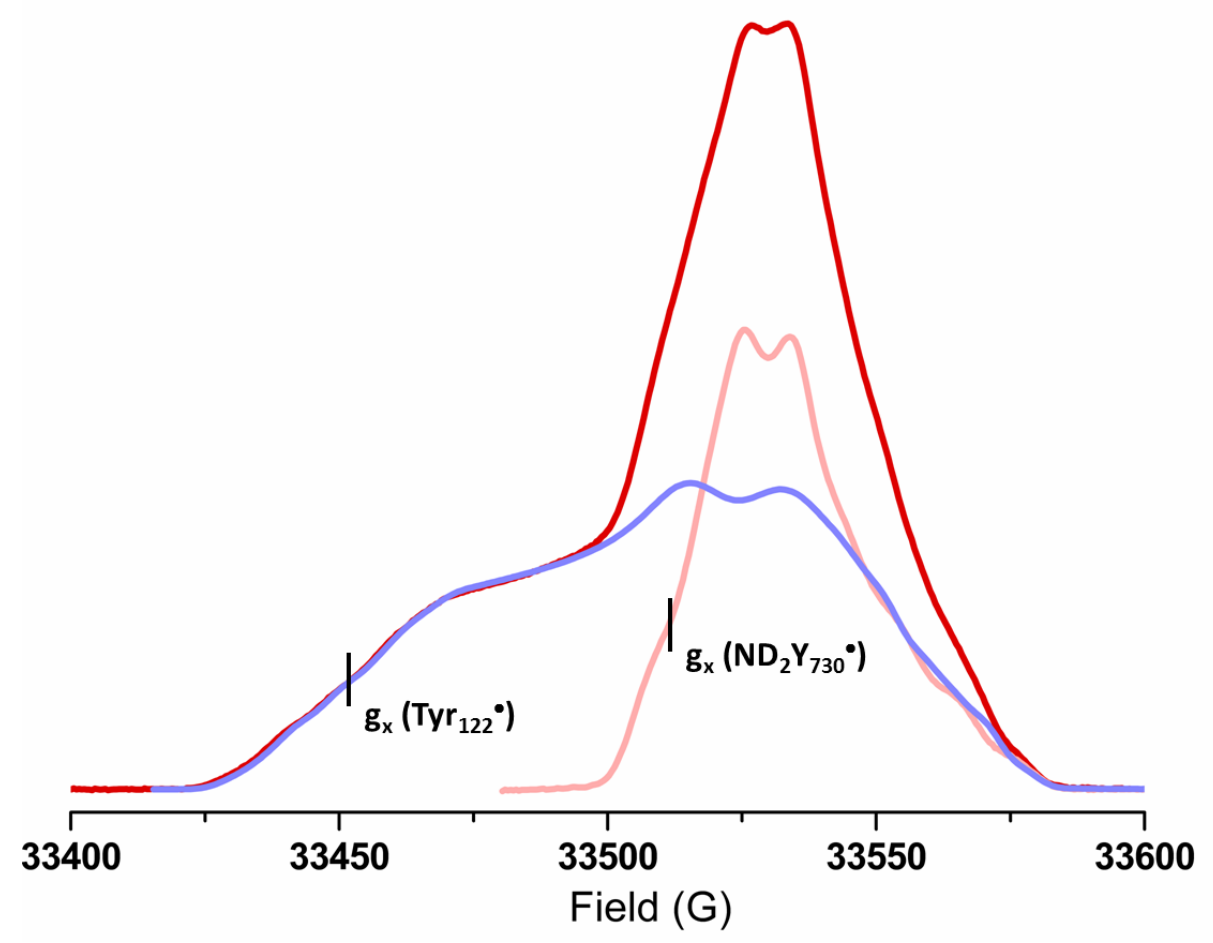

Figures 3.4. Decomposition of $\mathrm{Y}_{122}{ }^{\circ}$ and $\mathrm{ND}_{2} \mathrm{Y}_{730^{\circ}}$ at $94 \mathrm{GHz}$. A representative absorptive spectrum consisting of $\mathrm{ND}_{2} \mathrm{Y}_{730^{\circ}}$ and $\mathrm{Y}_{122}{ }^{\circ}$ is shown in the dark red spectrum which is measured at $10 \mathrm{~K}$. At $70 \mathrm{~K}$ only the $\mathrm{ND}_{2} \mathrm{Y}_{730^{\circ}}$ signal is present which is shown in the light red spectrum. The $\mathrm{Y}_{122}{ }^{\bullet}$ spectrum is shown in light purple. The amount of $\mathrm{ND}_{2} \mathrm{Y}_{730}{ }^{\circ}$ is $\approx 40 \%$ in the composite spectrum (quenching time $30-40 \mathrm{sec})$. The $\mathrm{g}_{\mathrm{x}}$ value in the composite spectrum on the low field side belongs to the $\mathrm{Y}_{122}{ }^{\circ}$ and is different from the $\mathrm{ND}_{2} \mathrm{Y}_{730^{\circ}}$ one, expressing hereby a different hydrogen-bond environment. The intrinsic $\mathrm{Y}_{122} \cdot$ with $\mathrm{g}_{\mathrm{x}}=2.0091, \mathrm{~g}_{\mathrm{y}}=2.0046$ and $\mathrm{g}_{\mathrm{z}}=2.00225$ has been used as an internal standard in order to get out the g-values for the new $\mathrm{ND}_{2} \mathrm{Y}^{\bullet}$ species. Experimental conditions (dark red trace): $\mathrm{T}=70 \mathrm{~K}, \mathrm{MW}(\pi / 2)=32 \mathrm{~ns}, \tau=260 \mathrm{~ns}, \mathrm{MW}(\pi)=64 \mathrm{~ns}$, repetition time $=3 \mathrm{~ms}$, shots $/$ point $=50$, scans $=700$. Experimental conditions (light red trace): $\mathrm{T}=10 \mathrm{~K}, \mathrm{MW}(\pi / 2)=32 \mathrm{~ns}, \tau=340 \mathrm{~ns}$, $\mathrm{MW}(\pi)=64 \mathrm{~ns}$, repetition time $=40 \mathrm{~ms}$, shots $/$ point $=20$, scans $=125$.

\section{Electron spin echo spectroscopy at $94 \mathrm{GHz}$ of $\alpha-\mathrm{NH}_{2} \mathrm{Y}_{730^{\circ}}$ and $\alpha-\mathrm{NH}_{2} \mathrm{Y}_{731}$ in $\mathrm{H}_{2} \mathrm{O}$ and $\mathrm{D}_{2} \mathrm{O}$ assay buffer}

In order to understand the hyperfine couplings associated with the $\mathrm{NH}_{2}$ protons, pulsed $94 \mathrm{GHz}$ EPR absorptive spectra were recorded at $70 \mathrm{~K}$ for $\mathrm{NH}_{2} \mathrm{Y}_{730 / 731}$ in $\mathrm{H}_{2} \mathrm{O}$ and $\mathrm{D}_{2} \mathrm{O}$. Afterwards the point-by-point first derivative was computed (Figure 3-5 and 3.6). All spectra show highly resolved anisotropic hyperfine interactions for some of the nuclei involved at 94 $\mathrm{GHz}$ and can be interpreted with simple splitting schemes when recorded in $\mathrm{D}_{2} \mathrm{O}$ buffer. 
In case of the $\mathrm{NH}_{2} \mathrm{Y}_{730^{\circ}}$ in $\mathrm{D}_{2} \mathrm{O}$ the spectrum is dominated by two large hyperfine couplings, one almost isotropic and a second largely anisotropic. At the high-field edge $\left(B|| g_{z}\right)$, both hyperfine couplings are large with a similar size, giving rise to a quartet with 1:2:2:1 intensity. In the center and at the low field side $\left(\mathrm{B}|| \mathrm{g}_{\mathrm{y}}\right.$ and $\left.\mathrm{B}|| \mathrm{g}_{\mathrm{x}}\right)$ of the $94 \mathrm{GHz}$ spectrum only one large hyperfine coupling is detected.

For the $\mathrm{NH}_{2} \mathrm{Y}_{731}{ }^{\circ}$ spectrum in $\mathrm{D}_{2} \mathrm{O}$ we can observe at the low field side at $\mathrm{B}|| \mathrm{g}_{\mathrm{x}}$ a doublet of doublet arising from a minor and a larger hyperfine coupling. In the center at $\mathrm{B}|| \mathrm{g}_{\mathrm{y}}$ a smaller hyperfine coupling is detected than in case of $\mathrm{NH}_{2} \mathrm{Y}_{730}{ }^{\circ}$ at this position and at $\mathrm{B}|| \mathrm{g}_{\mathrm{z}}$ in the quartet pattern region an additional peak is visible.

A large anisotropic interaction is typical for the ${ }^{14} \mathrm{~N}$ nucleus which has major spin density in the $p_{z}$ orbital (Figure 3-2 upper inset) $(17,18)$ and a large isotropic coupling is typical for $\beta$ methylene protons that have a $\mathrm{C}-\mathrm{H}$ bond oriented almost parallel to the $\mathrm{p}_{z}$ orbital of $\mathrm{C} 1$ in the aromatic ring (Figure 3-2 lower inset), as has been well documented for $\mathrm{Y}_{122}{ }^{\bullet}$ of E.coli RNR and other $\mathrm{Y}^{\circ} \mathrm{S}$ (19-21). In case of the $\mathrm{NH}_{2} \mathrm{Y}_{731}{ }^{\circ}$ the doublet of doublet splitting pattern in the low-field region arises through different dihedral angles for the $C_{\beta}$-methylene protons which place the $\mathrm{C}-\mathrm{H}$ bonds in two different positions. The different methylene proton couplings for $\mathrm{NH}_{2} \mathrm{Y}_{730}{ }^{\circ}$ and $\mathrm{NH}_{2} \mathrm{Y}_{731}{ }^{\circ}$ are also visible in Q-band ENDOR spectra measured for both radicals at $70 \mathrm{~K}$ in $\mathrm{D}_{2} \mathrm{O}$ buffer (shown below).

The spectra in $\mathrm{H}_{2} \mathrm{O}$ show a substantially different line shape, which arises from hyperfine contributions of the $\mathrm{NH}_{2}$ protons. The complexity of these spectra is such that the visible features can not be interpreted with simple splitting schemes. The spectra have therefore to be analyzed through iterative simulations at 9 and $94 \mathrm{GHz}$ taking also the g-values into account which are the same for both radicals at $180 \mathrm{GHz}$. 


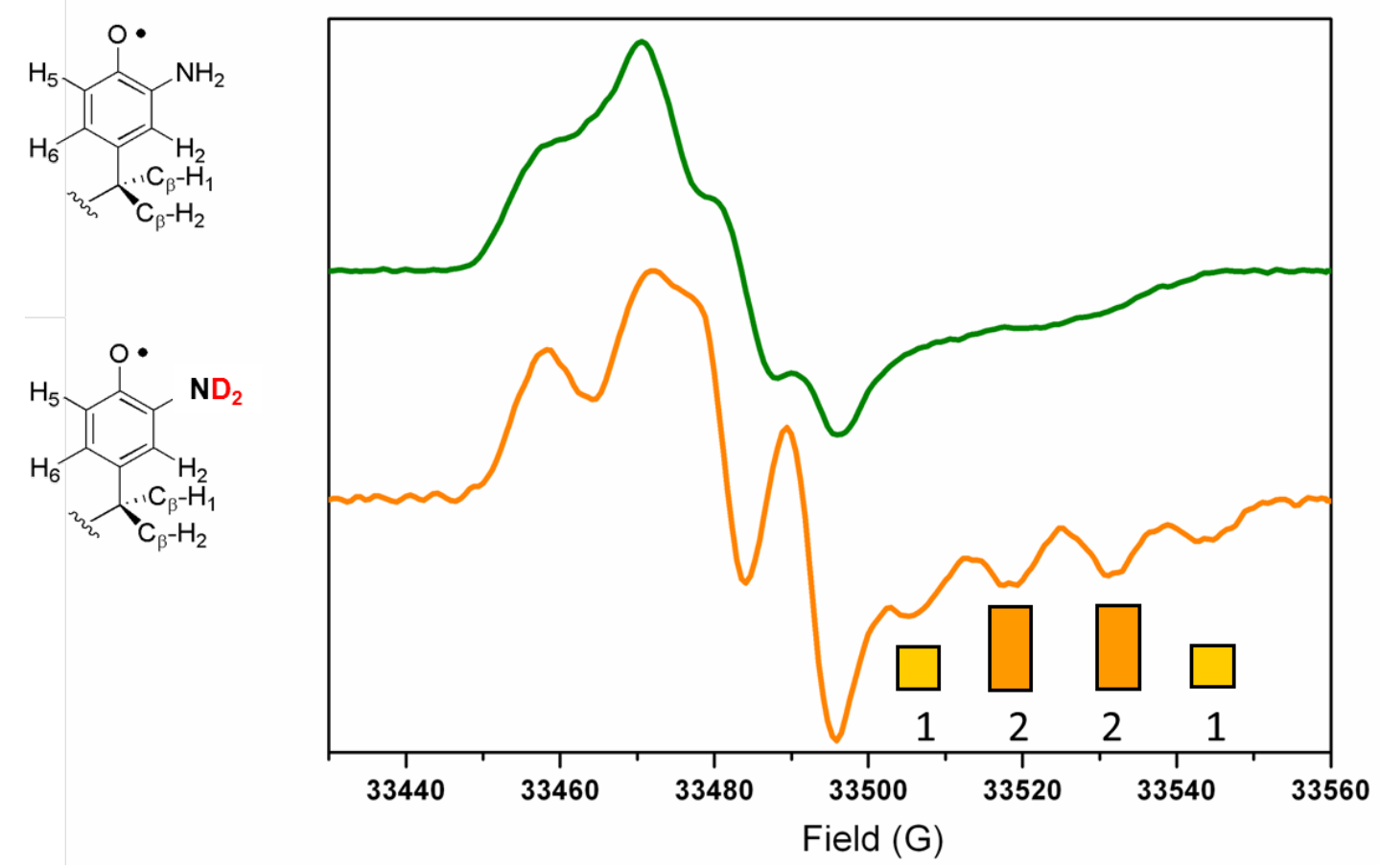

Figures 3.5. Comparison of $94 \mathrm{GHz}$ pulsed derivative EPR spectra of $\mathrm{NH}_{2} \mathrm{Y}_{730}{ }^{\bullet}$ in $\mathrm{H}_{2} \mathrm{O}$ (green trace) and $\mathrm{D}_{2} \mathrm{O}$ (orange trace) assay buffer, respectively. Notice the profound change in line shape after deuteration. The quartett splitting pattern in the high field region comes from the coupling of the electron to the ${ }^{14} \mathrm{~N}$ nucleus $(S=1)$ and one proton of the $C_{\beta}$-methylen group.

Experimental conditions (upper spectrum): $\mathrm{T}=70 \mathrm{~K}, \mathrm{MW}(\pi / 2)=32 \mathrm{~ns}, \tau=248 \mathrm{~ns}, \mathrm{MW}(\pi)=64 \mathrm{~ns}$, repetition time $=5 \mathrm{~ms}$, shots $/$ point $=50$, scans $=700$. Experimental conditions (lower spectrum): $\mathrm{T}=$ $70 \mathrm{~K}, \mathrm{MW}(\pi / 2)=32 \mathrm{~ns}, \tau=260 \mathrm{~ns}, \mathrm{MW}(\pi)=64 \mathrm{~ns}$, repetition time $=3 \mathrm{~ms}$, shots $/$ point $=50$, scans $=$ 700. 


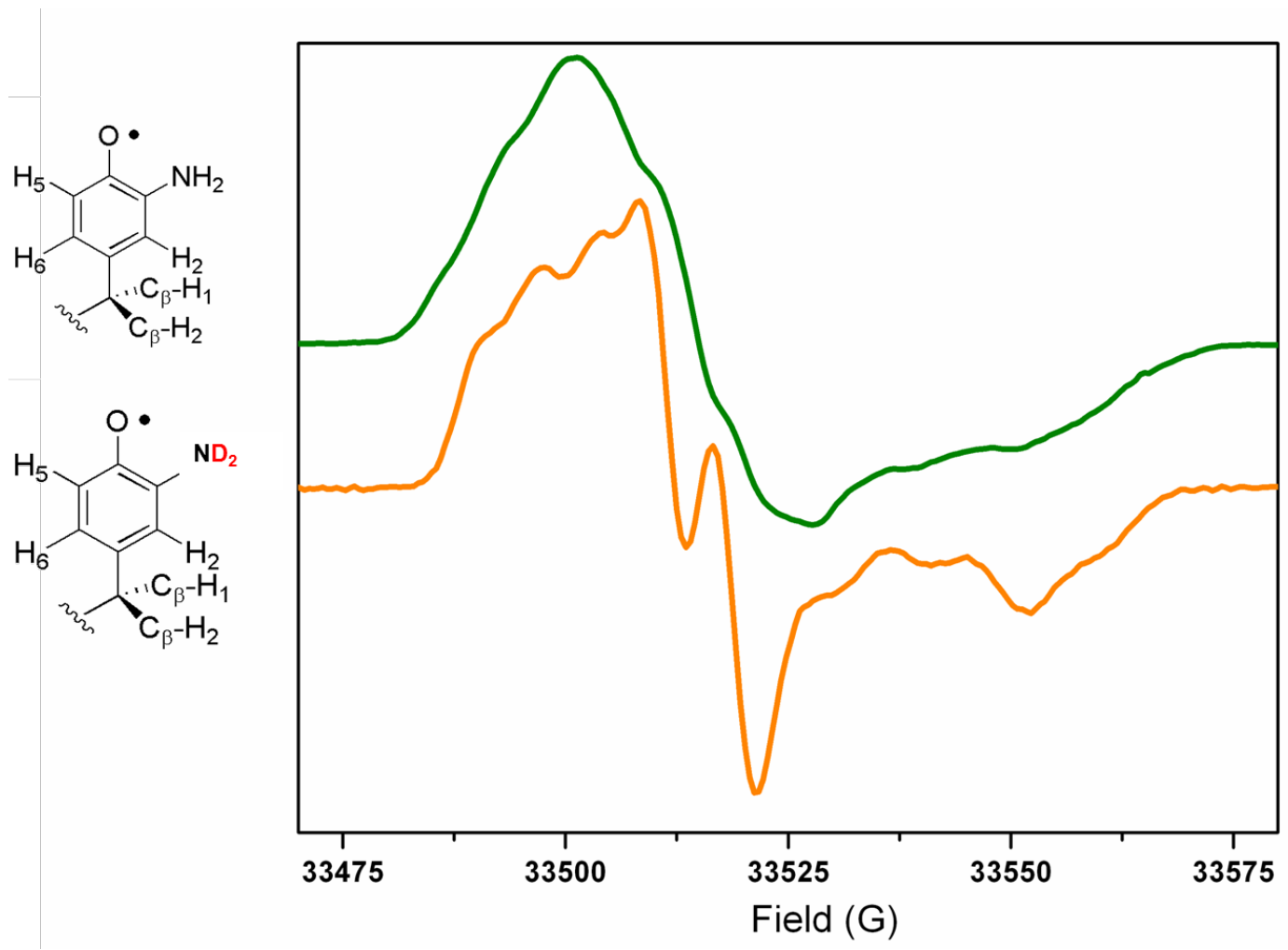

Figures 3.6. Comparison of $94 \mathrm{GHz}$ pulsed derivative EPR spectra of $\mathrm{NH}_{2} \mathrm{Y}_{731} \cdot$ in $\mathrm{H}_{2} \mathrm{O}$ (green trace) and $D_{2} \mathrm{O}$ (orange trace) assay buffer respectively. Notice the additional peak appearing in the high field region in $\mathrm{D}_{2} \mathrm{O}$ spectrum of $\mathrm{NH}_{2} \mathrm{Y}_{731}$. Experimental conditions (upper spectrum): $\mathrm{T}=70 \mathrm{~K}, \mathrm{MW}(\pi / 2)=$ $20 \mathrm{~ns}, \tau=248 \mathrm{~ns}, \mathrm{MW}(\pi)=40 \mathrm{~ns}$, repetition time $=5 \mathrm{~ms}$, shots $/$ point $=50$, scans $=1300$.

Experimental conditions (lower spectrum): $\mathrm{T}=70 \mathrm{~K}, \mathrm{MW}(\pi / 2)=16 \mathrm{~ns}, \tau=260 \mathrm{~ns}, \mathrm{MW}(\pi)=32 \mathrm{~ns}$, repetition time $=5 \mathrm{~ms}$, shots $/$ point $=50$, scans $=1070$.

The additional peak which appears in the $\mathrm{D}_{2} \mathrm{O}$ spectrum of $\mathrm{NH}_{2} \mathrm{Y}_{731}{ }^{\bullet}$ is most probably originating from a second radical species. The detection of spectra at two different time points demonstrates a diminishing of the radical species in the second time scale

(Figure 3-7). If this radical species is the $\mathrm{NH}_{2} \mathrm{Y}_{731}{ }^{\bullet}$ in another conformation on the radical propagation pathway or another radical species (i.e. $Y^{\bullet}$ ) is not clear until now. Work in order to quench the radical species on the millisecond time scale is in progress. However it is known from $X$-ray structure studies on $\alpha-\mathrm{NH}_{2} \mathrm{Y}_{731}$ which crystallizes as a trimer that in one monomer at position 731 the amino acid exhibits conformational flexibility. This conformational flexibility could be essential for the activity of E.coli RNR and can cause perturbations of redox potentials in the near environment of $\alpha-\mathrm{NH}_{2} \mathrm{Y}_{731}$. However until today the flexibility of this residue was not observed in PELDOR studies. 


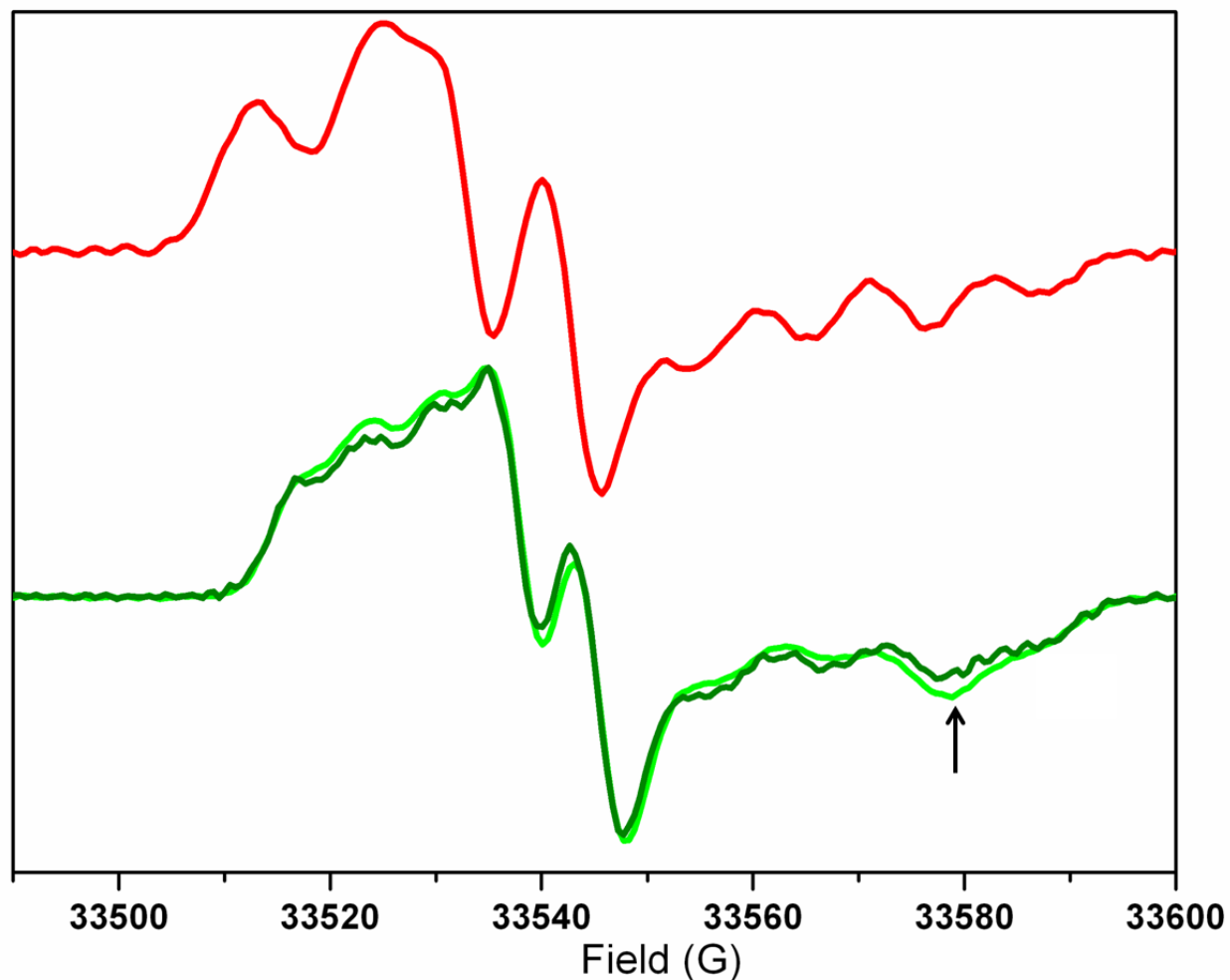

Figures 3.7. Time course of the potentially new radical in the $\mathrm{NH}_{2} \mathrm{Y}_{731}{ }^{\circ}$ spectrum is shown for two time points (lower part). The light green EPR spectrum is recorded after 30 seconds quenching time and the dark green spectrum after 60 seconds. The arrow indicates the diminishing intensity of the new radical species. The quartet pattern appears hereby at the latest time point. For comparison the spectrum at the top of the figure shows the $\mathrm{NH}_{2} \mathrm{Y}_{730^{\circ}}$.

Proton Davies ENDOR spectroscopy at $34 \mathrm{GHz}$ of $\alpha-\mathrm{NH}_{2} \mathrm{Y}_{730^{\circ}}$ and $\alpha-\mathrm{NH}_{2} \mathrm{Y}_{731}{ }^{\circ}$ in $\mathrm{D}_{2} \mathrm{O}$ assay buffer

${ }^{1} \mathrm{H}$ Davies ENDOR spectra have been recorded in $\mathrm{D}_{2} \mathrm{O}$ buffer in order to detect the hyperfine couplings for $\mathrm{C}_{\beta}$-methylene protons of $\mathrm{NH}_{2} \mathrm{Y}_{730}$ and $\mathrm{NH}_{2} \mathrm{Y}_{731}{ }^{\circ}$ (Figure 3-8 and 3-9). After the exchange of the amino protons through deuterons large coupling contributions which are visible in the Davies spectrum should originate primarily from methylene protons.

In case of the $\mathrm{ND}_{2} \mathrm{Y}_{730^{\circ}}$ one can observe already by eye a major isotropic hyperfine value, which is in the range of $30 \mathrm{MHz}$. This value can be assigned to one $\mathrm{C}_{\beta}$-methylene proton, based also on a DFT study of the $p$-ethylphenoxy radical (see Appendix Figure A-4) where the dependence of the $C_{\beta}$-methylene proton coupling is calculated with respect to the $C_{\alpha}-C_{\beta}$ ring-plane dihedral angle (22). For the second $\mathrm{C}_{\beta}$-methylene proton one gets out an isotropic 
hyperfine value of $\sim 5 \mathrm{MHz}$ and from both hyperfine values a $\mathrm{C}_{\alpha}-\mathrm{C}_{\beta}$-ring-plane dihedral angle of $60^{\circ}$ results. In addition to the theoretical results the $30 \mathrm{MHz}$ coupling could be confirmed in the simulation of the $\mathrm{NH}_{2} \mathrm{Y}_{730}{ }^{\circ}$ in $\mathrm{D}_{2} \mathrm{O}$ buffer (shown below in the next section).

In the Davies ENDOR spectrum of $\mathrm{NH}_{2} \mathrm{Y}_{730^{\circ}}$ one observes additional proton hyperfine couplings which are below $10 \mathrm{MHz}$. They originate from ring protons at the $\mathrm{C} 2, \mathrm{C} 5$ and $\mathrm{C} 6$ position based on DFT calculations of the $o$-aminophenol radical (23). The hyperfine coupling of the second $\mathrm{C}_{\beta}$-methylene proton appears also in this region but is not resolved.

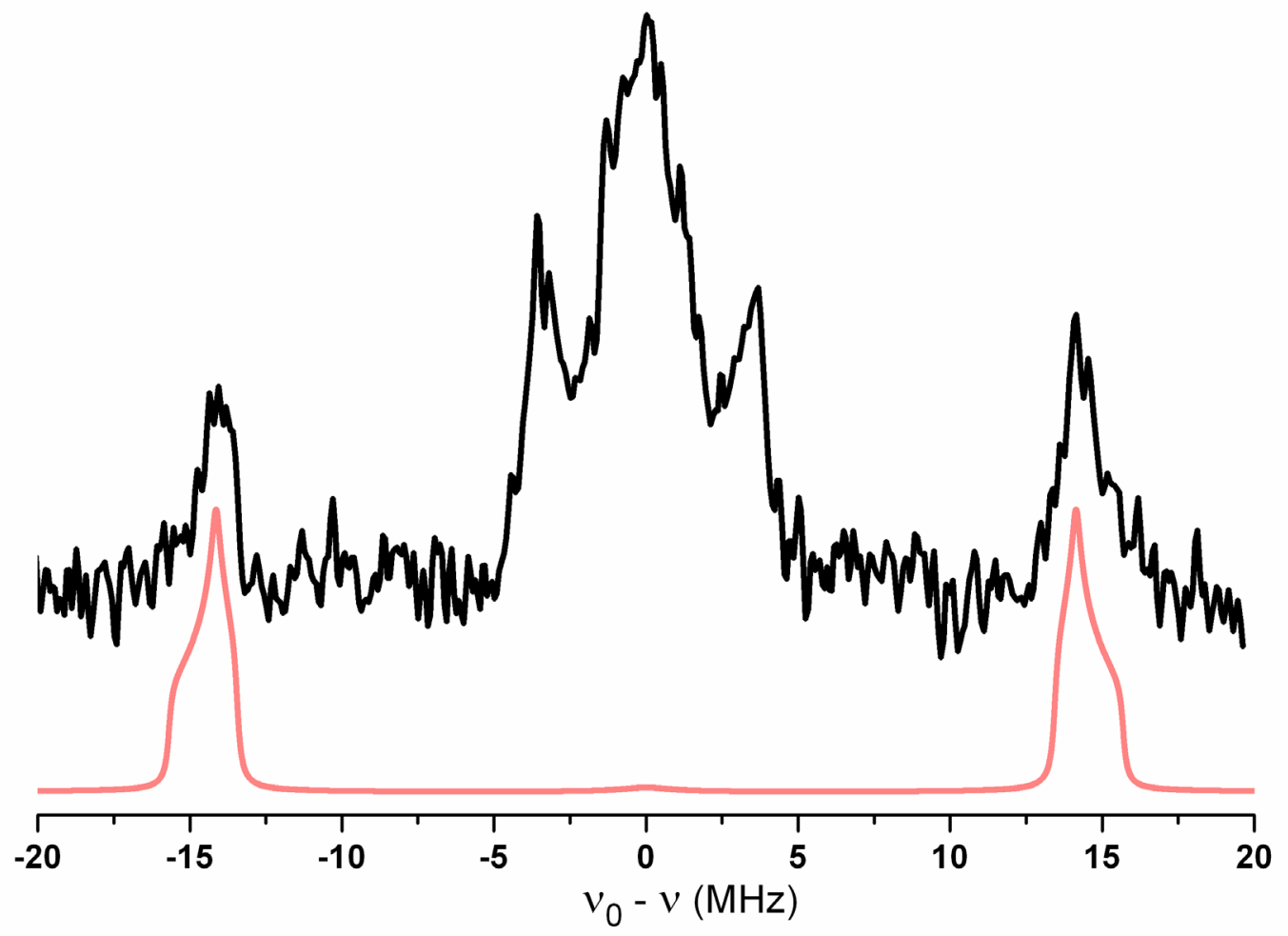

Figures 3.8. $34 \mathrm{GHz}{ }^{1} \mathrm{H}$ Davies ENDOR on $\alpha-\mathrm{NH}_{2} \mathrm{Y}_{730^{\circ}}$ in $\mathrm{D}_{2} \mathrm{O}$ assay buffer (black). The large proton hyperfine coupling could be simulated with the hyperfine tensor for one methylene proton which is also used for the simulation of the $1^{\text {st }}$ derivative EPR spectrum in $D_{2} \mathrm{O}$ buffer. Experimental conditions: $\mathrm{T}=70 \mathrm{~K}, \mathrm{MW}(\pi / 2)=20 \mathrm{~ns}$, prep $(\pi)=128 \mathrm{~ns}, \tau=300 \mathrm{~ns}$, repetition time $=5 \mathrm{~ms}$, $\mathrm{RF}$ pulse $=15 \mu \mathrm{s}$, scans $=15100,72 \mathrm{~h}$ acquisition time.

In the ${ }^{1} \mathrm{H}$ Davies ENDOR spectrum of $\mathrm{NH}_{2} \mathrm{Y}_{731}{ }^{\bullet}$ we can observe one proton hyperfine coupling which is around $20 \mathrm{MHz}$ and another one which is around $10 \mathrm{MHz}$. They can be confirmed through the before mentioned DFT calculation on the p-ethylphenoxy radical and are assigned to both $C_{\beta}$-methylene protons. From these two hyperfine couplings a $C_{\alpha}-C_{\beta}$-ring$\mathbf{9 2} \mid \mathrm{P}$ a g e 
plane dihedral angle of $80^{\circ}$ results. Furthermore the two hyperfine values for the methylene protons are approved through the simulation of the $\mathrm{NH}_{2} \mathrm{Y}_{731}{ }^{\circ}$ in $\mathrm{D}_{2} \mathrm{O}$ buffer (shown below in the next section). The rest of the Davies ENDOR spectrum is related to resonances of the ring protons as explained before.

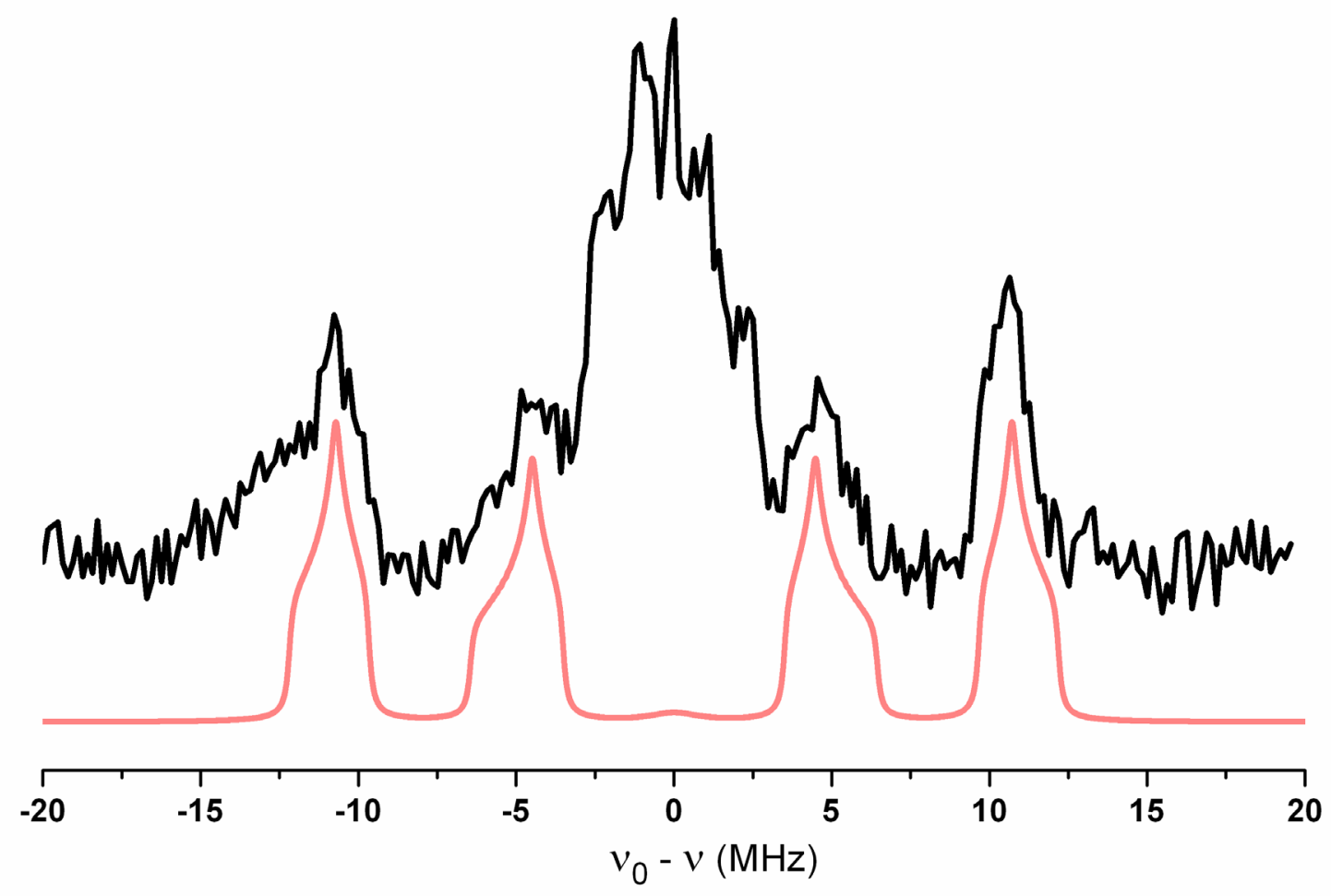

Figures 3.9. $34 \mathrm{GHz}{ }^{1} \mathrm{H}$ Davies ENDOR on $\alpha-\mathrm{NH}_{2} \mathrm{Y}_{731}{ }^{\circ}$ in $\mathrm{D}_{2} \mathrm{O}$ assay buffer (black). The two proton hyperfine couplings could be simulated with the parameters of two hyperfine tensors which are also used for the simulation of the $1^{\text {st }}$ derivative EPR spectrum in $\mathrm{D}_{2} \mathrm{O}$ buffer. Experimental conditions: $\mathrm{T}=70 \mathrm{~K}, \mathrm{MW}(\pi / 2)=28 \mathrm{~ns}$, prep $(\pi)=128 \mathrm{~ns}, \tau=300 \mathrm{~ns}$, repetition time $=5 \mathrm{~ms}$, RF pulse $=15 \mu \mathrm{s}$, scans $=15149,72 \mathrm{~h}$ acquisition time.

\section{Spectral simulations of $\alpha-\mathrm{NH}_{2} \mathrm{Y}_{730}{ }^{\circ}$ and $\alpha-\mathrm{NH}_{2} \mathrm{Y}_{731}{ }^{\circ}$ in $\mathrm{H}_{2} \mathrm{O}$ and $\mathrm{D}_{2} \mathrm{O}$ assay buffer at 9 and} $94 \mathrm{GHz}$

The simulation strategy for the analysis of the $\mathrm{NH}_{2} \mathrm{Y}_{730} \cdot$ required an analysis of the spectra at different frequencies (multifrequency approach) where the constraints posed by the spectra were determined independently and then combined to find a global solution. The g-values which were determined by $180 \mathrm{GHz}$ EPR spectroscopy were held as fixed parameters 
throughout all 9 and $94 \mathrm{GHz}$ simulations. The simulation in deuterated buffer medium was attempted first where the hyperfine couplings to two nuclei were included in the simulations. One large hyperfine coupling was assumed to be isotropic, what is typical for $\mathrm{C}_{\beta}$-methylene protons and the second one was set anisotropic with the largest tensor component at $A_{z z}$ which is typical for a ${ }^{14} \mathrm{~N}$ nucleus which has major spin density in the $p_{z}$-orbital. Due to a spin of $\mathrm{I}=1$ the ${ }^{14} \mathrm{~N}$ nucleus generates a triplet pattern. The hyperfine couplings of the exchangeable amino deuterons however are very small $\left(A_{\text {iso }}<5 \mathrm{MHz}\right)$ and disappear in the EPR line width which is $11-13 \mathrm{MHz}$ at 9 and $94 \mathrm{GHz}$. The largest hyperfine coupling of the ring protons is around $10 \mathrm{MHz}$ and contributes consequently to the total line width of $11-13 \mathrm{MHz}$. Simulations in $\mathrm{D}_{2} \mathrm{O}$ buffer were performed in parallel at both frequencies ( 9 and $94 \mathrm{GHz}$ ) and led after iterative rounds to a satisfactory result (Figure 3-10 and 3-11). The hyperfine tensor for the $C_{\beta}$-methylene protons was found to have a small anisotropy, which is approved also in the $34 \mathrm{GHz}$ ENDOR spectrum and was previously found for $\mathrm{Y}_{122}{ }^{\circ}$ at $140 \mathrm{GHz}(24)$. In case of the ${ }^{14} \mathrm{~N}$ parameters a $5^{\circ}-\beta$-angle and a $60^{\circ}$ - $\alpha$-angle had to be introduced. The two angles are related to the position of the $\mathrm{C}-\mathrm{N}$-bond within the g-Tensor frame and are in agreement with the $5^{\circ}$ out-of-plane position of the bond and a $60^{\circ}$ in-plane rotation away from the C-O bond (see Figure 3-3 on the left side). The relevancy of the Euler angles will be explained below in detail for the position of the two $\mathrm{N}-\mathrm{H}$ bonds when it comes to the analysis of the spectra in $\mathrm{H}_{2} \mathrm{O}$ buffer. 


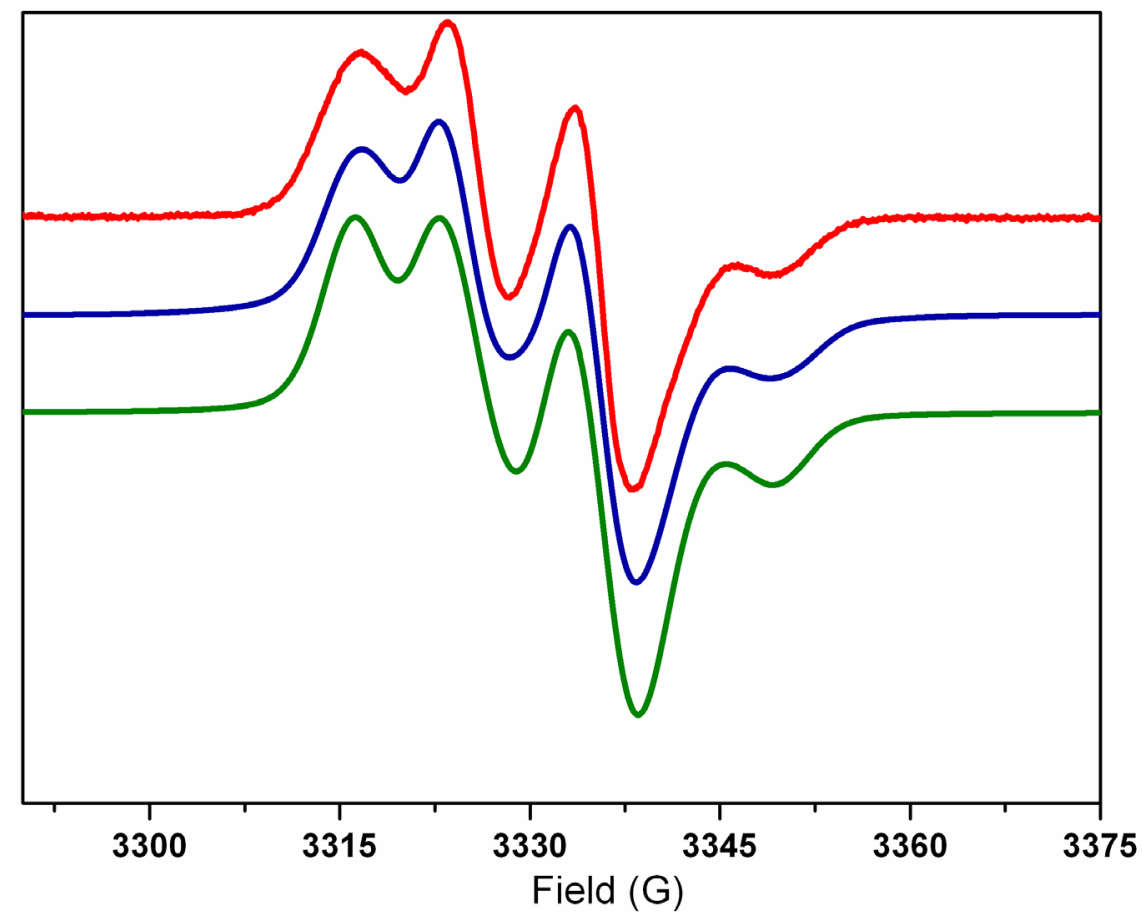

Figure 3-10. Simulations $($ blue $=$ simulation software SimFonia; green $=$ simulation software EasySpin) of the first derivative EPR spectrum (red) of $\mathrm{NH}_{2} \mathrm{Y}_{730^{\circ}}$ in $\mathrm{D}_{2} \mathrm{O}$ assay buffer at $9 \mathrm{GHz}$. The SimFonia software does not take into account forbidden transitions at $9 \mathrm{GHz}$ which slightly broaden the simulated spectrum. Therefore in addition the EasySpin software was used which includes these transitions. Another choice for the EasySpin software was the possibility to use a fitting procedure with the genetic algorithm. Only a slight difference could be observed between the two simulations. See Table 3-1 for the summary of simulation parameters. In order to rationalize the Euler rotation and the Euler angles within the context of a structure see section on page 98. 


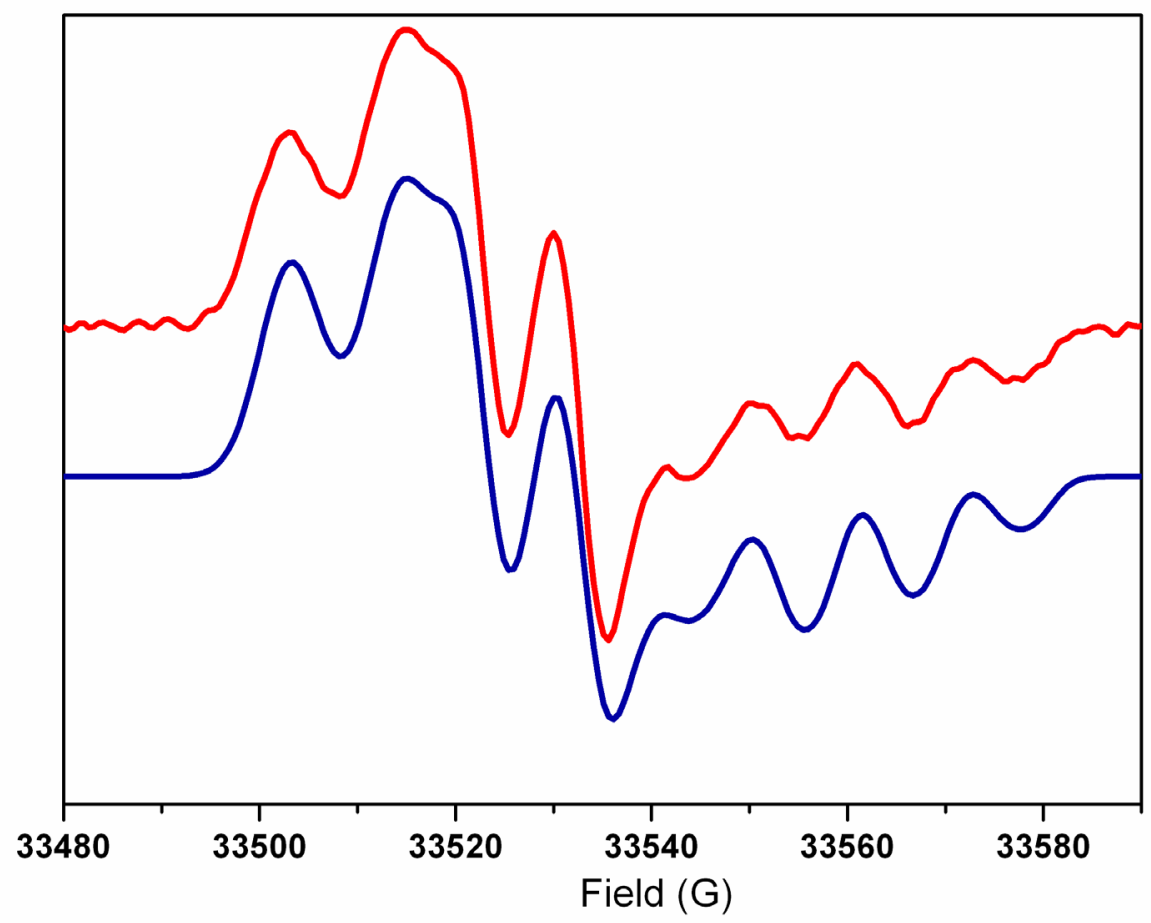

Figure 3-11. Simulation (blue = SimFonia) of the first derivative EPR spectrum (red) of $\mathrm{NH}_{2} \mathrm{Y}_{730}{ }^{\circ}$ in $\mathrm{D}_{2} \mathrm{O}$ assay buffer at $94 \mathrm{GHz}$. See Table 3-1 for the summary of simulation parameters. In order to rationalize the Euler rotation and the Euler angles see section on page 98.

The analysis of the spectra in $\mathrm{H}_{2} \mathrm{O}$ buffer was attempted next at $94 \mathrm{GHz}$ including the parameters obtained from the simulation in $\mathrm{D}_{2} \mathrm{O}$ buffer. To start the simulation procedure at $94 \mathrm{GHz}$ was reasonable, as it provides more constraints due to higher resolution than the $9 \mathrm{GHz}$ spectrum. Two additional couplings had to be included in the obtained parameter set from the simulations in $\mathrm{D}_{2} \mathrm{O}$ which are originating from the two protons of the amino group (Table 3-1). We assumed hereby that the hyperfine tensor axes of the amino protons were not collinear with the $g$ tensor axes (Figure 3-12) and performed the simulations by varying systematically all hyperfine tensor components in the specific field regions together with all Euler angles which were varied in $5-10^{\circ}$ steps. 


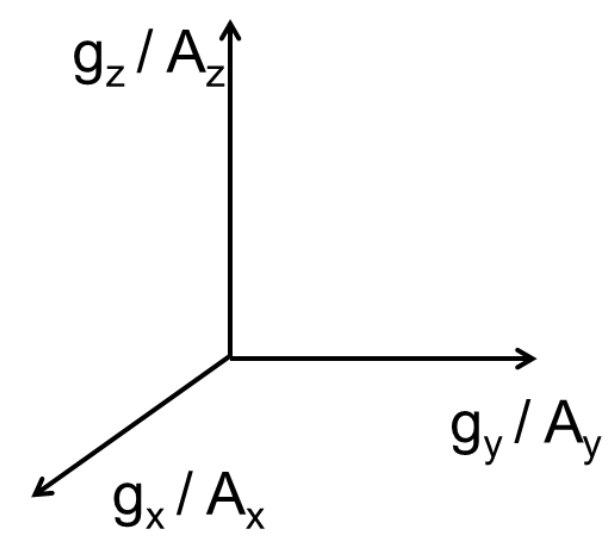

Coincidence of $\mathbf{g}$ and $\mathbf{A}$ tensor

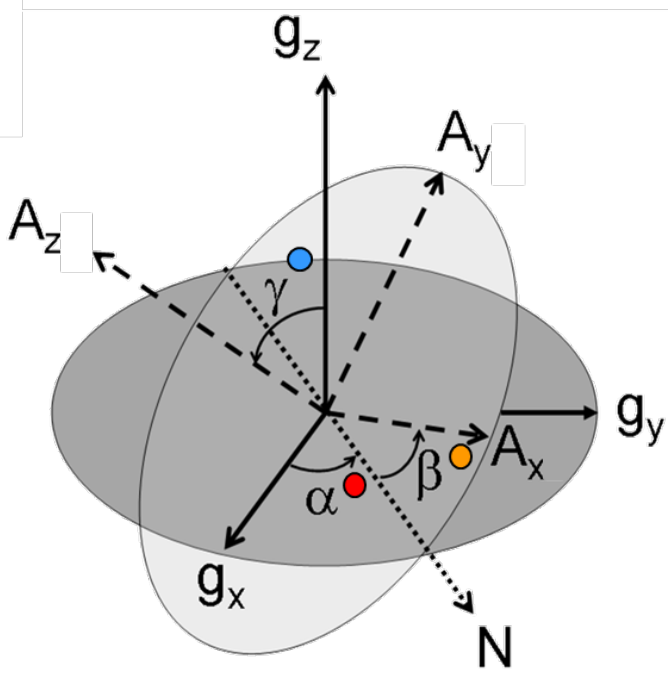

Noncoincidence of $\mathbf{g}$ and $\mathbf{A}$ tensor

Figure 3-12. Illustration of the A-tensor frame placed into the g-tensor frame from a situation of coincidence (left) to a situation of noncoincedence (right). Three Euler rotations are necessary around different A tensor axes in order to co-linearize both tensors (situation at the left side) using the Euler angles $\alpha, \beta$ and $\gamma . A_{x}$ points hereby into the bond direction (i.e. the $\mathrm{N}-\mathrm{H}$ bond of the aminogroup).

Our starting parameters for the two protons were based on the studies of the $o$-aminophenol radical where we started with a set of two isotropic hyperfine couplings of $15 \mathrm{MHz}$ and a set of Euler angles which accounted for the direction of the N-H bonds in the molecular frame. After several simulation rounds at $94 \mathrm{GHz}$ it became clear that we need non-equivalent hyperfine couplings for the protons with two anisotropic hyperfine tensors. The parameters were then optimized also in several simulation rounds through the simulation of the $9 \mathrm{GHz}$ spectrum and the solution for both spectra is displayed below (Figure 3-13 and 3-14). 


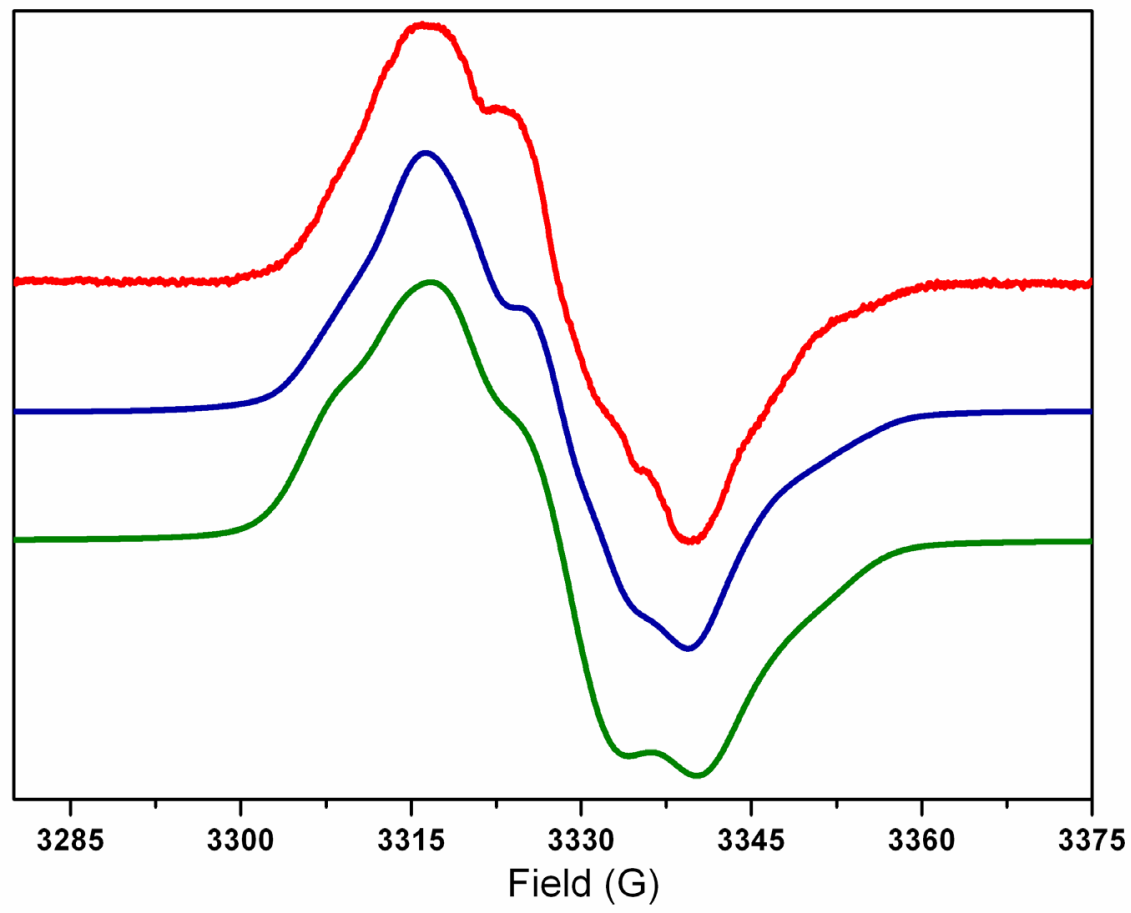

Figure 3-13. Simulations $($ blue $=$ simulation software SimFonia; green $=$ simulation software EasySpin) of the first derivative EPR spectrum (red) of $\mathrm{NH}_{2} \mathrm{Y}_{730^{\circ}}$ in $\mathrm{H}_{2} \mathrm{O}$ assay buffer at $9 \mathrm{GHz}$. See Table 3-1 for the summary of simulation parameters. In order to rationalize the Euler rotation and the Euler angles within the context of a structure see section on page 98.

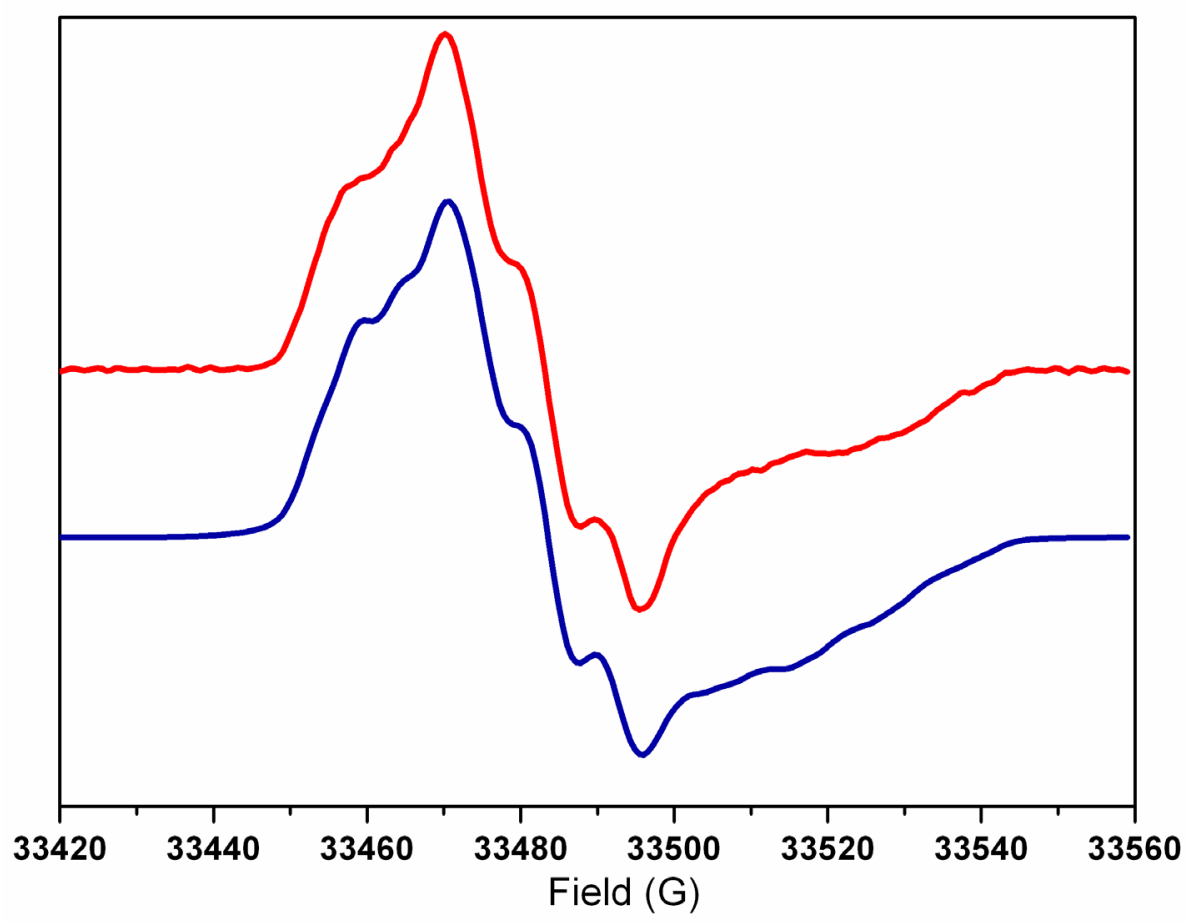


Figure 3-14. Simulation (blue $=$ SimFonia) of the first derivative EPR spectrum (red) of $\mathrm{NH}_{2} \mathrm{Y}_{730}{ }^{\circ}$ in $\mathrm{H}_{2} \mathrm{O}$ assay buffer at $94 \mathrm{GHz}$. See Table 3-1 for the summary of simulation parameters. In order to rationalize the Euler rotation and the Euler angles within the context of a structure see section on page 98.

The parameter set obtained from the study of the $\mathrm{NH}_{2} \mathrm{Y}_{730}{ }^{\circ}$ was used as a starting set for the simulation of $\mathrm{NH}_{2} \mathrm{Y}_{731} 1^{\circ}$. The spectrum in $\mathrm{D}_{2} \mathrm{O}$ was simulated with two different hyperfine couplings for the $C_{\beta}$-methylene protons which were already visible in the Davies ENDOR study (see section before). However the anisotropic hyperfine tensor for the ${ }^{14} \mathrm{~N}$ nucleus was not changed and the additional peak at $33610 \mathrm{G}$ was not taken into account. Minor modifications (up to $4 \mathrm{MHz}$ ) had to be performed for the $A_{z z}$ hyperfine tensor values of both amino-protons and the $A_{x x}$ hyperfine tensor value of $\mathrm{H} 2$ of the $\mathrm{NH}_{2}$-group had to be changed from 13 to $18.2 \mathrm{MHz}$. However the Euler angles which express the conformation of the $\mathrm{NH}_{2}$-group and the g-values which are sensitive to the electrostatic environment (i.e hydrogen bond network) were not changed at all. The simulations at $94 \mathrm{GHz}$ in $\mathrm{D}_{2} \mathrm{O}$ and $\mathrm{H}_{2} \mathrm{O}$ buffer are plotted under the experimental spectra and are shown below (Figure 3-15 and 3-16).

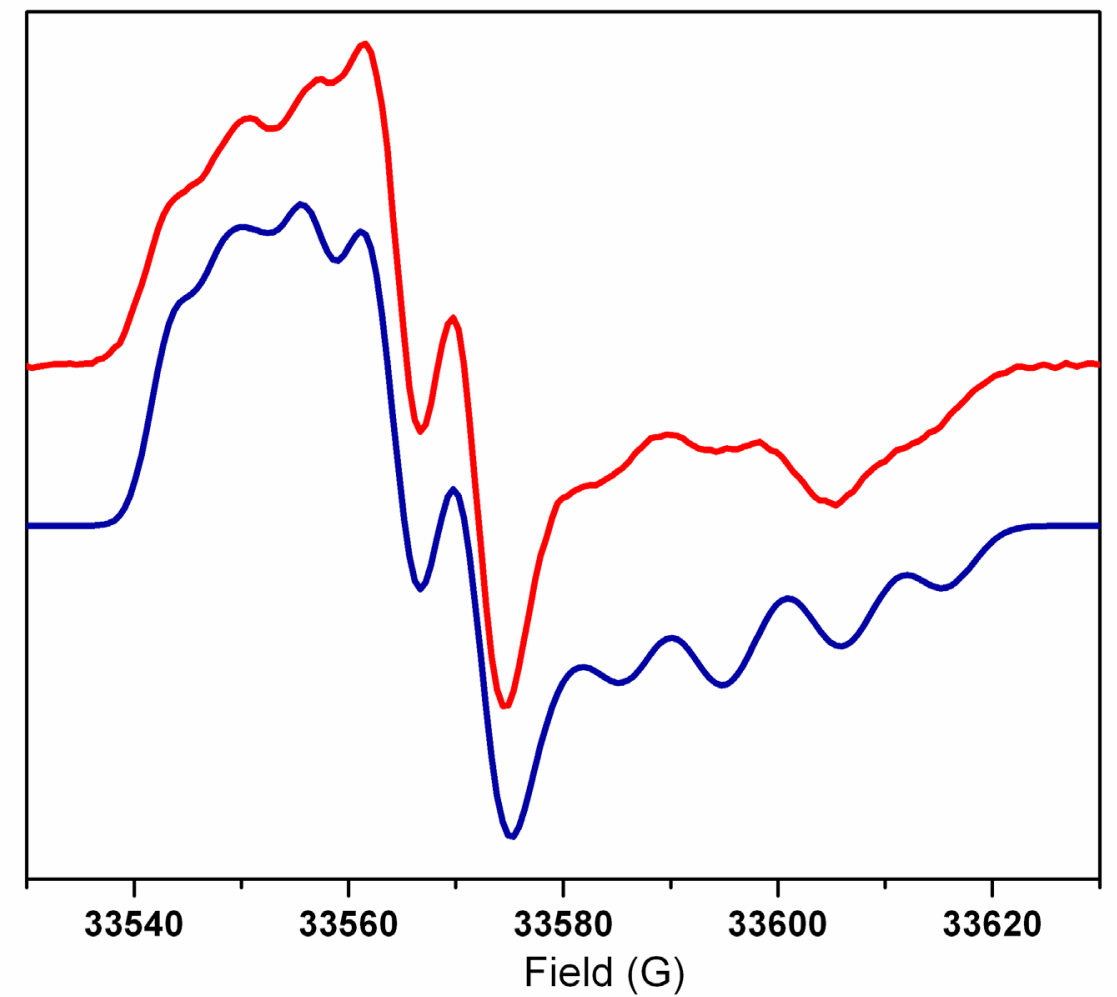


Figure 3-15. Simulation (blue = simulation software SimFonia) of the first derivative EPR spectrum (red) of $\mathrm{NH}_{2} \mathrm{Y}_{731}{ }^{\circ}$ in $\mathrm{D}_{2} \mathrm{O}$ assay buffer at $94 \mathrm{GHz}$. See Table 3-1 for summary of simulation parameters. In order to rationalize the Euler rotation and the Euler angles within the context of a structure see section on page 98 .

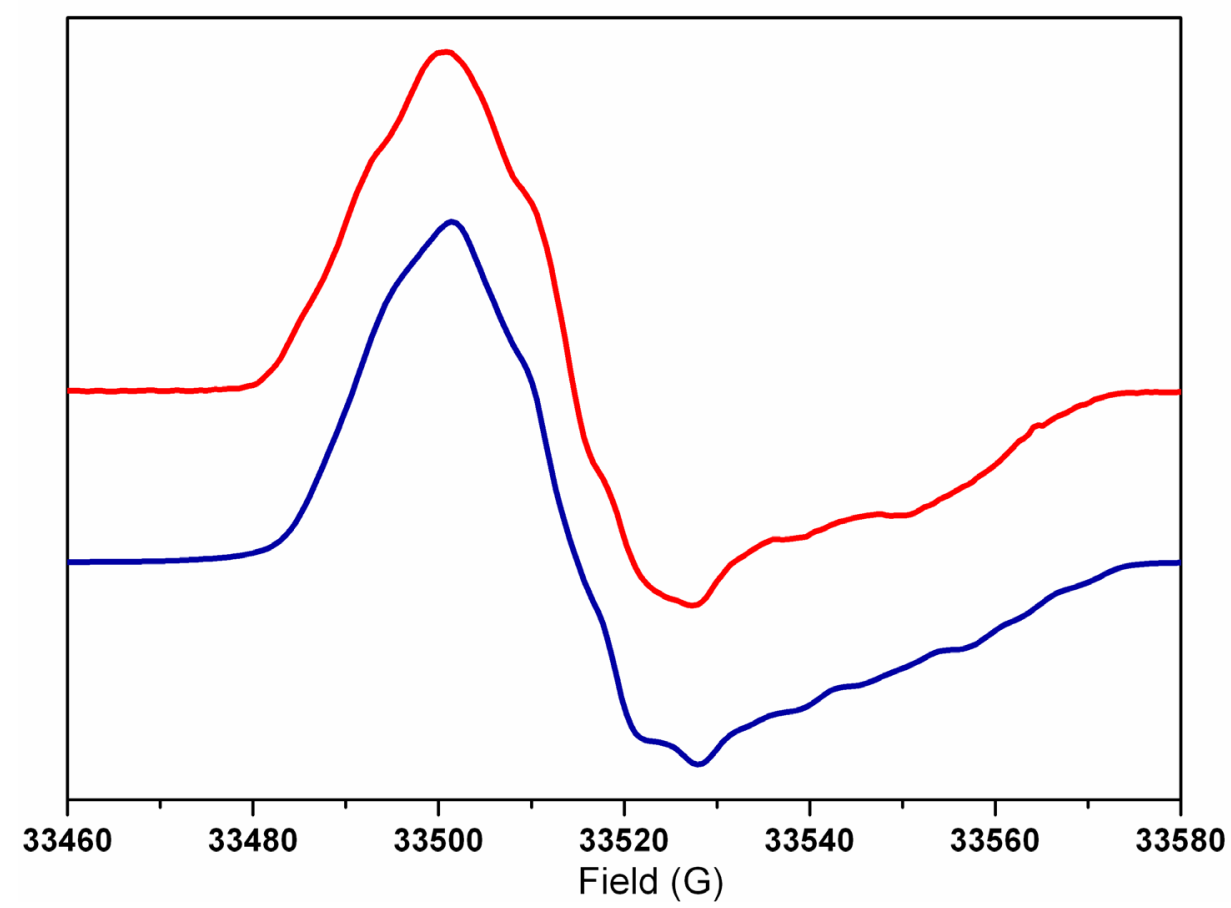

Figure 3-16. Simulation (blue = simulation software SimFonia) of the first derivative EPR spectrum (red) of $\mathrm{NH}_{2} \mathrm{Y}_{731}{ }^{\circ}$ in $\mathrm{H}_{2} \mathrm{O}$ assay buffer at $94 \mathrm{GHz}$. See Table 3-1 for the summary of simulation parameters. In order to rationalize the Euler rotation and the Euler angles within the context of a structure see section on page 98 .

$\alpha-\mathrm{NH}_{2} \mathrm{Y}_{730}{ }^{\circ}$ and $\alpha-\mathrm{NH}_{2} \mathrm{Y}_{731}{ }^{\circ}$ spectra at $34 \mathrm{GHz}$ together with their simulations are shown in the Appendix (Figure A-5, A-6, A-7).

\section{Spectral simulations of $\beta-\mathrm{NH}_{2} \mathrm{Y}_{356}{ }^{\circ}$ in $\mathrm{D}_{2} \mathrm{O}$ and $\mathrm{H}_{2} \mathrm{O}$ assay buffer at $94 \mathrm{GHz}$ and comparison of all $\mathrm{NH}_{2} \mathrm{Y}^{\bullet}$ on the radical propagation pathway}

We have also performed simulations for the newly generated $\mathrm{NH}_{2} \mathrm{Y}^{\bullet}$ at position 356 in the $\beta$-subunit. As starting parameters we used again the set from the simulation of the $\mathrm{NH}_{2} \mathrm{Y}_{730^{\circ}}$ where we optimized the hyperfine values for one $C_{\beta}$-methylene proton and the $A_{y y}$ value of the $\mathrm{H} 1$ of the $\mathrm{NH}_{2}$-group. The change of the $A_{y y}$ value from 8 to $15 \mathrm{MHz}$ was necessary in 
order to simulate the line shape in the $g_{y}$ region (between 33620 and $33640 \mathrm{G}$ ). However the hyperfine tensor for the ${ }^{14} \mathrm{~N}$ nucleus was nearly the same as it was used for the simulations of $\mathrm{NH}_{2} \mathrm{Y}_{730^{\circ}}$ and $\mathrm{NH}_{2} \mathrm{Y}_{731}$ ' which forms the previously mentioned quartet pattern in the high field region. Also in this case the Euler angles had not to be changed at all but we observed a slight shift in the $g_{x}$ and $g_{y}$ values to 2.0050 and 2.0041 respectively, indicating a more hydrophilic environment around the $\mathrm{NH}_{2} \mathrm{Y}^{\bullet}$. The two simulations in $\mathrm{D}_{2} \mathrm{O}$ and $\mathrm{H}_{2} \mathrm{O}$ buffer are displayed below (Figure 3-17 and 3-18).

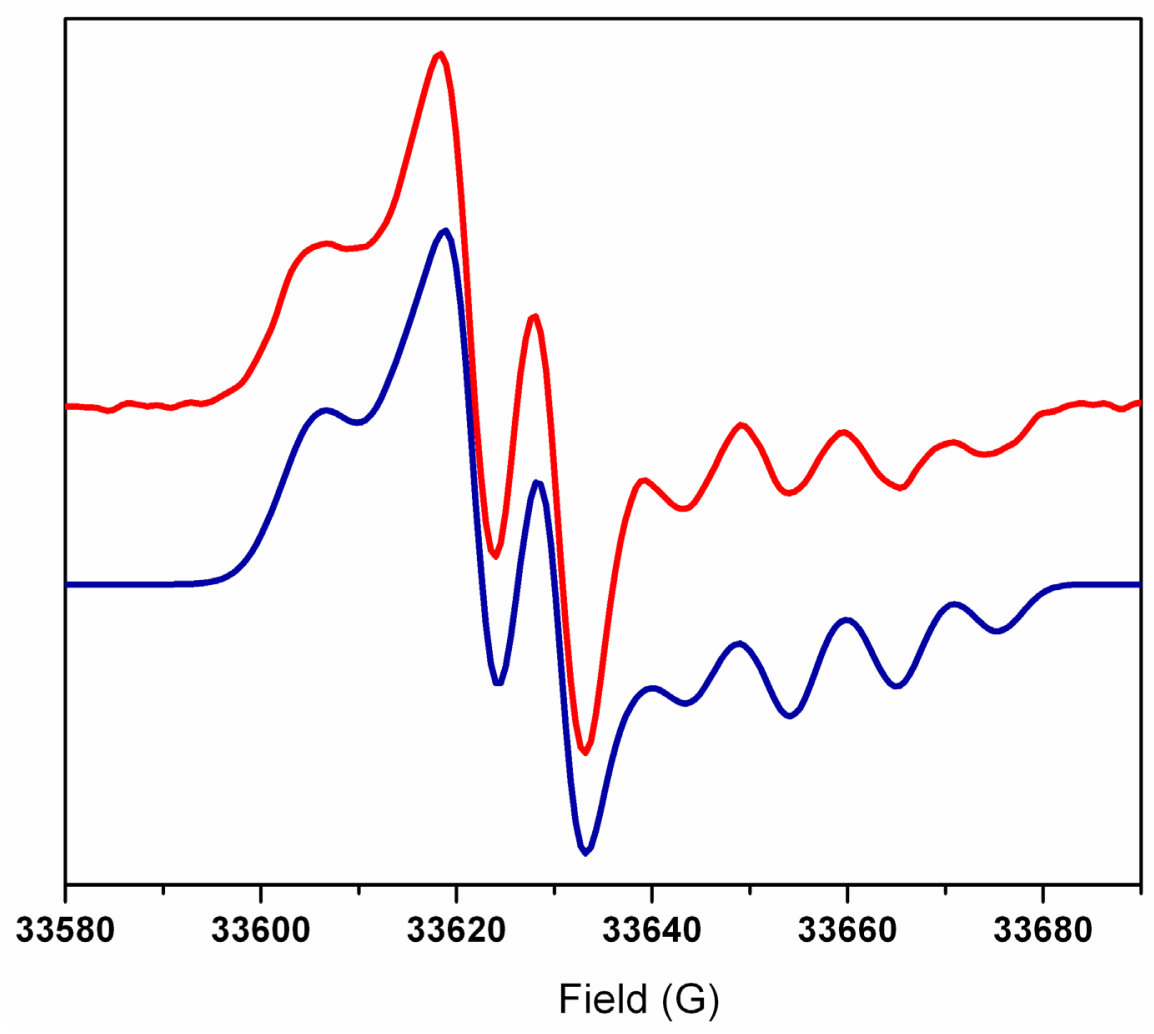

Figure 3-17. Simulation (blue = simulation software SimFonia) of the first derivative EPR spectrum (red) of $\beta-\mathrm{NH}_{2} \mathrm{Y}_{356^{\circ}}$ in $\mathrm{D}_{2} \mathrm{O}$ assay buffer at $94 \mathrm{GHz}$. See Table 3-1 for the summary of simulation parameters. In order to rationalize the Euler rotation and the Euler angles within the context of a structure see section on page 98. Experimental conditions: $\mathrm{T}=70 \mathrm{~K}, \mathrm{MW}(\pi / 2)=16 \mathrm{~ns}, \tau=260 \mathrm{~ns}$, $\mathrm{MW}(\pi)=32 \mathrm{~ns}$, repetition time $=5 \mathrm{~ms}$, shots $/$ point $=50$, scans $=583$. 


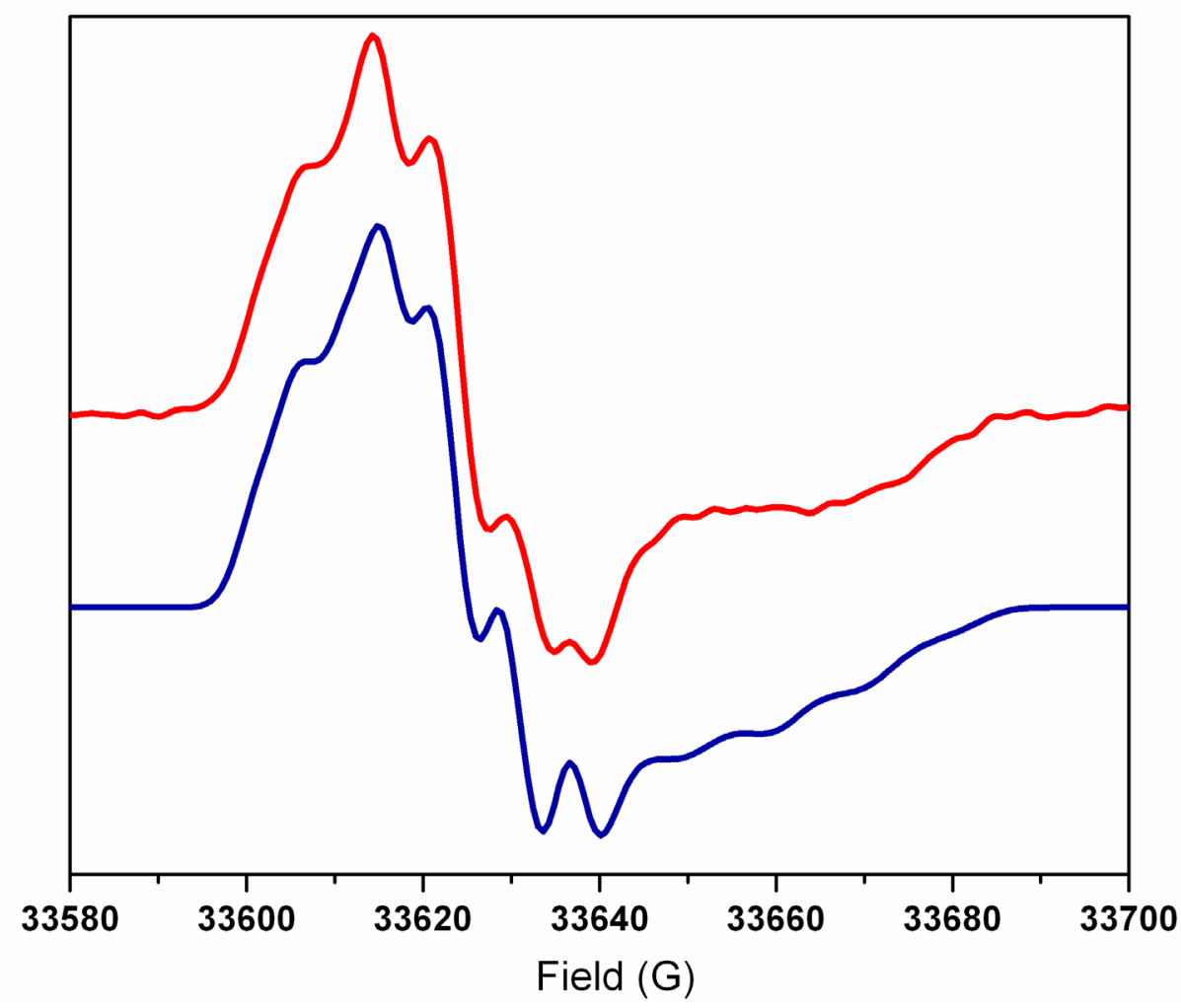

Figure 3-18. Simulation (blue = simulation software SimFonia) of the first derivative EPR spectrum (red) of $\beta-\mathrm{NH}_{2} \mathrm{Y}_{356}{ }^{\circ}$ in $\mathrm{H}_{2} \mathrm{O}$ assay buffer at $94 \mathrm{GHz}$. See Table 3-1 for the summary of simulation parameters. In order to rationalize the Euler rotation and the Euler angles within the context of a structure see section on page 98. Experimental conditions: $\mathrm{T}=70 \mathrm{~K}, \mathrm{MW}(\pi / 2)=32 \mathrm{~ns}, \tau=260 \mathrm{~ns}$, $\mathrm{MW}(\pi)=64 \mathrm{~ns}$, repetition time $=5 \mathrm{~ms}$, shots $/$ point $=200$, scans $=750$.

All EPR parameters for each single $\mathrm{NH}_{2} \mathrm{Y}^{\bullet}$ on the radical propagation pathway are summarized below (Table 3-1). What is most remarkable is that the ${ }^{14} \mathrm{~N}$ couplings and the proton couplings are very similar for each mutation site with one exception which is the 7 $\mathrm{MHz}$ difference for the $\mathrm{A}_{\mathrm{yy}}$ value of the $\mathrm{NH}_{2}(\mathrm{H} 1)$ of the $\mathrm{NH}_{2} \mathrm{Y}_{356}{ }^{\circ}$ when compared to the other two $\mathrm{NH}_{2} \mathrm{Y}^{\bullet}$ s. Still all used Euler angles in the simulations of the $\mathrm{NH}_{2} \mathrm{Y}^{\bullet}$ s are the same and the summarized EPR parameters for the $\mathrm{NH}_{2}$-group reflect a nearly identical $\mathrm{NH}_{2}$-conformation at all three mutation sites. The methylene couplings of the $\mathrm{NH}_{2} \mathrm{Y}^{\circ} \mathrm{s}$ show however a pronounced discrepancy from one mutation site to the other, which is clearly the case for position 731 . These couplings depend therefore on the specific location where the unnatural amino acid is incorporated. 


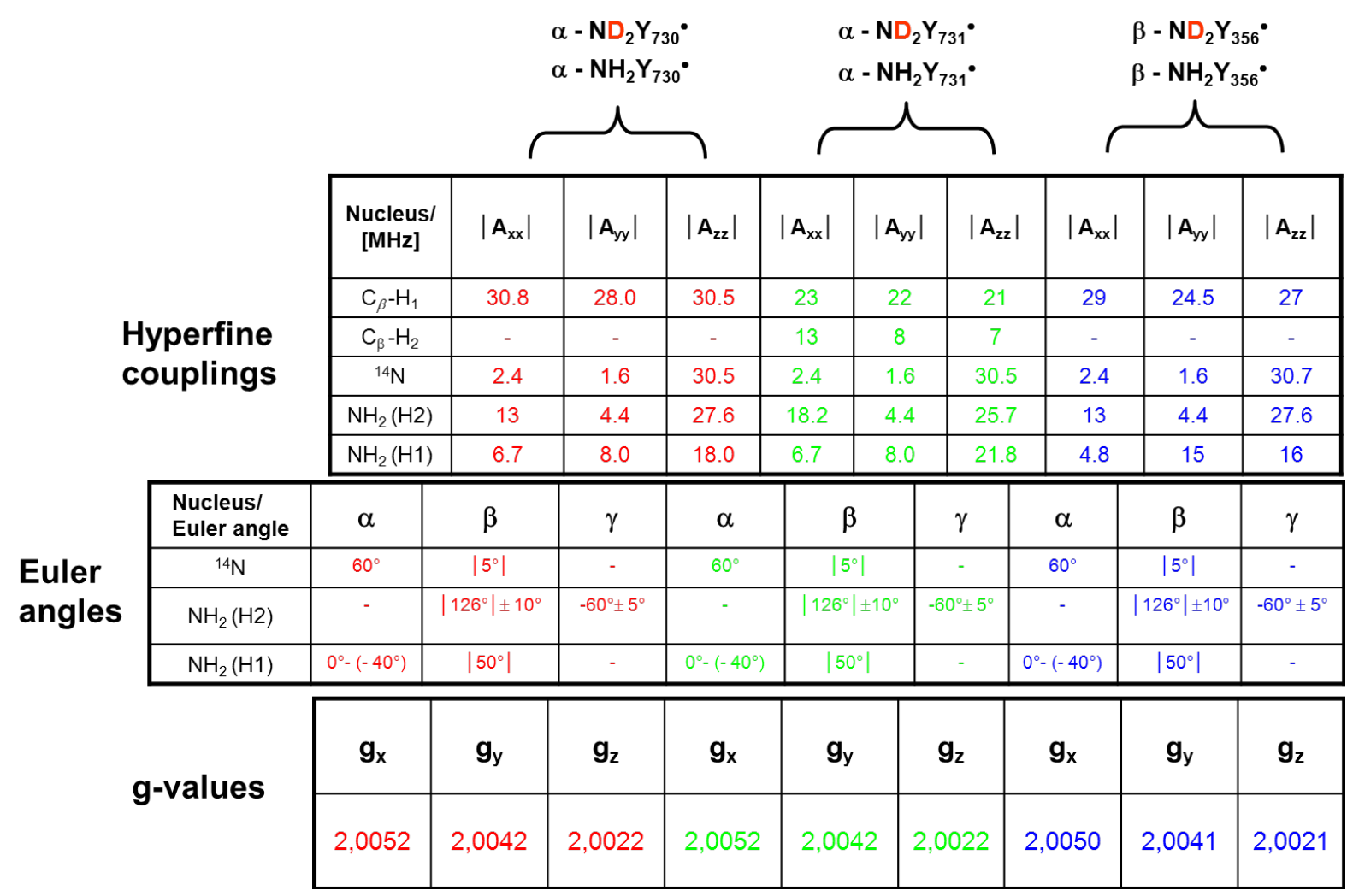

Table 3-1. Summary of all EPR parameters which were used in the simulation of $\mathrm{NH}_{2} \mathrm{Y}^{\circ} \mathrm{S}$ in $\mathrm{D}_{2} \mathrm{O}$ and $\mathrm{H}_{2} \mathrm{O}$ buffer at position $\beta-356, \alpha-731$ and $\alpha-730$ on the radical propagation pathway of E.coli RNR.

Presented are the hyperfine couplings, the Euler angles and the g-values. Isotropic hyperfine values are calculated according to the equation $A_{i s o}=\left(A_{x x}+A_{y y}+A_{z z}\right) / 3$.

All the simulations end up in structural information of the $\mathrm{NH}_{2} \mathrm{Y}^{\bullet}$ at position $\beta-356, \alpha-730$ and $\alpha-731$ which will be explained in the next section. The parameter set which is needed for an accurate analysis of all high-field EPR spectra is huge and needed to be determined as far as possible through the simulation of different EPR spectra which were measured in separate experiments. A detailed analysis of the intensities, line shapes and splitting patterns hereby taking also line broadenings into account was an imperative which lead finally to simulations which are in agreement with the experimental spectra. At the end an overview of all three measured $\mathrm{NH}_{2} \mathrm{Y}^{\circ}$ s in $\mathrm{D}_{2} \mathrm{O}$ and $\mathrm{H}_{2} \mathrm{O}$ buffer is shown. 


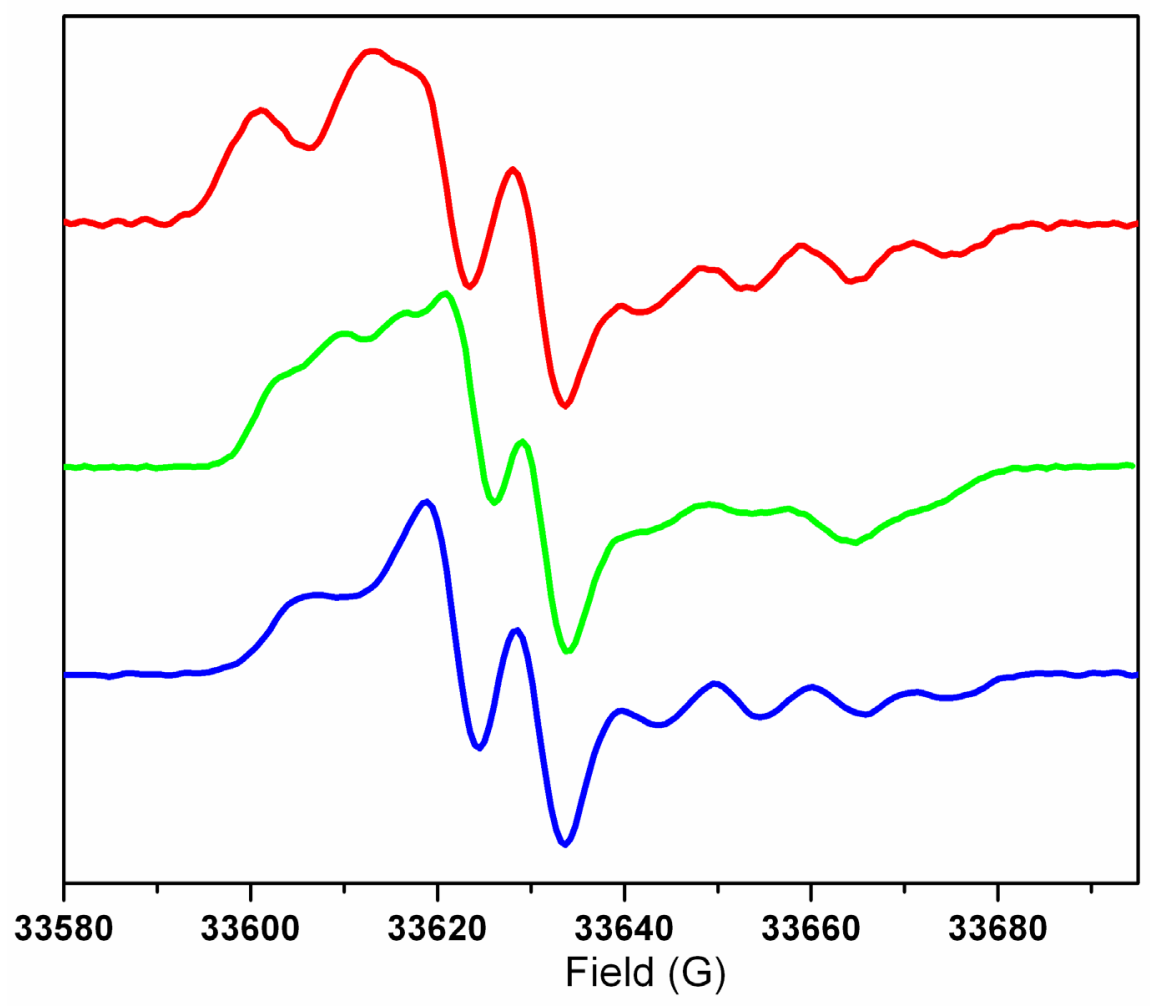

Figure 3-19. Comparison of all $1^{\text {st }}$ derivative $\mathrm{NH}_{2} \mathrm{Y}^{\bullet}$ spectra recorded in $\mathrm{D}_{2} \mathrm{O}$ buffer starting from the top with $\alpha-\mathrm{NH}_{2} \mathrm{Y}_{730}{ }^{\circ}$ (red), $\alpha-\mathrm{NH}_{2} \mathrm{Y}_{731}{ }^{\bullet}$ (green) and $\beta-\mathrm{NH}_{2} \mathrm{Y}_{356}{ }^{\bullet}$ (blue). The change in line shape is due to different $C_{\beta}$-methylene proton couplings. The quartet pattern at the high-field site is present for all $\mathrm{NH}_{2} \mathrm{Y}^{\circ} \mathrm{s}$, although slightly masked in case of $\mathrm{NH}_{2} \mathrm{Y}_{731}{ }^{\bullet}$ (due to the second radical species). 


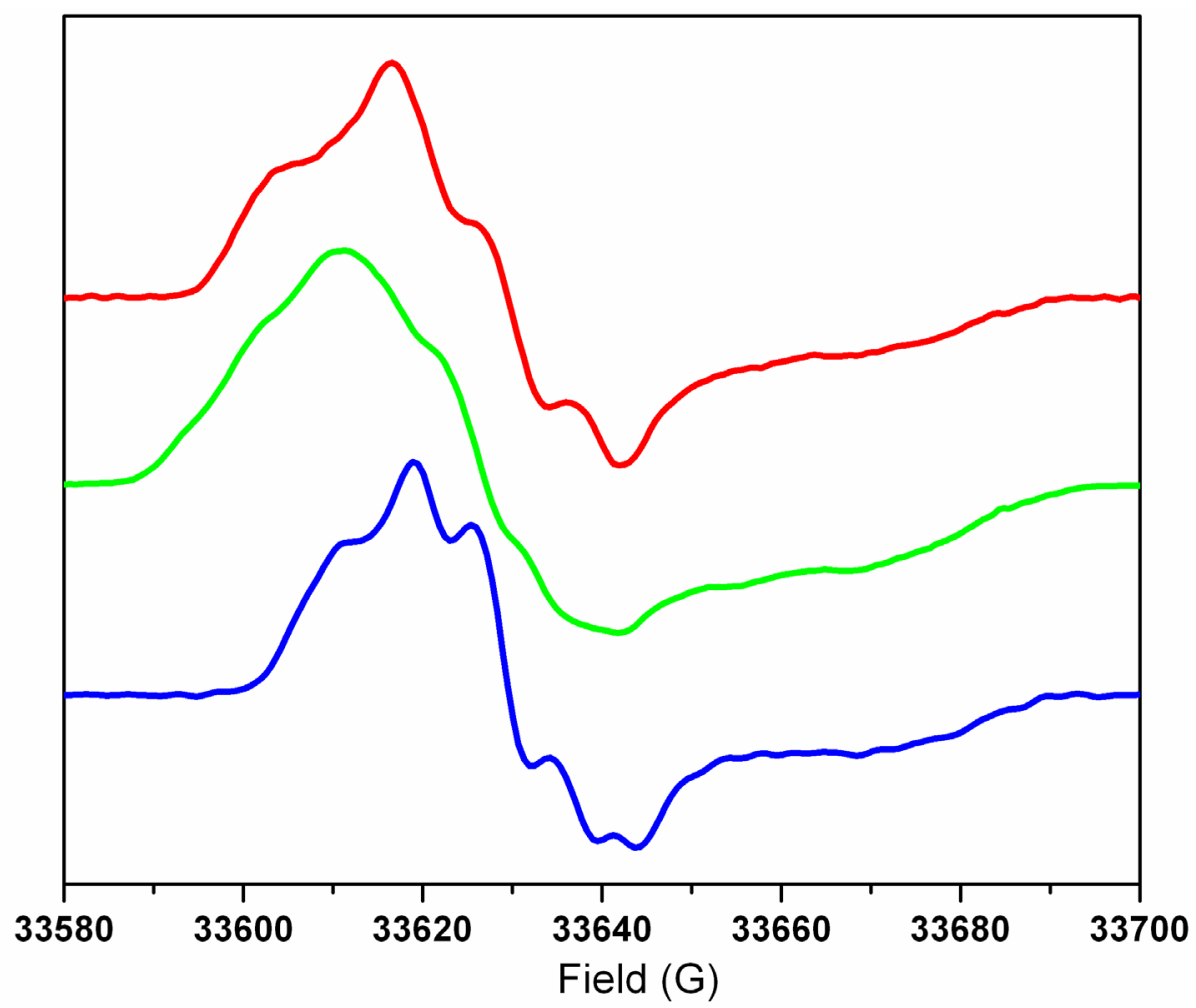

Figure 3-20. Comparison of all $1^{\text {st }}$ derivative $\mathrm{NH}_{2} \mathrm{Y}^{\bullet}$ spectra recorded in $\mathrm{H}_{2} \mathrm{O}$ buffer starting from the top with $\alpha-\mathrm{NH}_{2} \mathrm{Y}_{730}{ }^{\circ}$ (red), $\alpha-\mathrm{NH}_{2} \mathrm{Y}_{731}{ }^{\bullet}$ (green) and $\beta-\mathrm{NH}_{2} \mathrm{Y}_{356}{ }^{\bullet}$ (blue). An additional line shape which results from hyperfine couplings of the two amino-protons is filling up the quartet pattern at the high-field site and the doublet patterns at the low-field site. The overall discrepancy in line shape of the three spectra results still from different $C_{\beta}$-methylene proton couplings.

\section{Structure of $\mathrm{NH}_{2} \mathrm{Y}^{\bullet}$ at position $\beta-356, \alpha-731$ and $\alpha-730$}

In order to understand the results obtained from Table 3-1 it is necessary to establish the relationship between the obtained Euler angles which account for the rotation of the $A$ into the g-tensor frame and the molecular structure of the $\mathrm{NH}_{2} \mathrm{Y}^{\bullet}$. The g-tensor lies hereby with the $g_{x}$-axes in the $\mathrm{C}-\mathrm{O}$ bond, $\mathrm{g}_{z}$ orthogonal to the $\pi$-plane and $\mathrm{g}_{\mathrm{y}}$ orthogonal to the $\mathrm{g}_{\mathrm{x}}$ - and the $\mathrm{g}_{\mathrm{z}}$-axes. This is also confirmed through DFT calculations on the 4-ethyl-2-amino-tyrosyl radical (see Appendix Figure A-8). With this in hand we first rationalized the position of the $\mathrm{N}-\mathrm{H}(2)$ bond. The $A_{x}$-tensor axis lies along the bond and can be co-linearized with the $g_{x}{ }^{-}$ tensor axis after two consecutive rotations starting from the rotation around the $A_{y}$-axis with the Euler angle $\beta$ which $-126^{\circ}$ and afterwards with the rotation around the new $A_{z}$ axis $\left(A_{z}\right)$ with the Euler angle $\gamma$ which is $-60^{\circ}$ (Figure 3-21). 

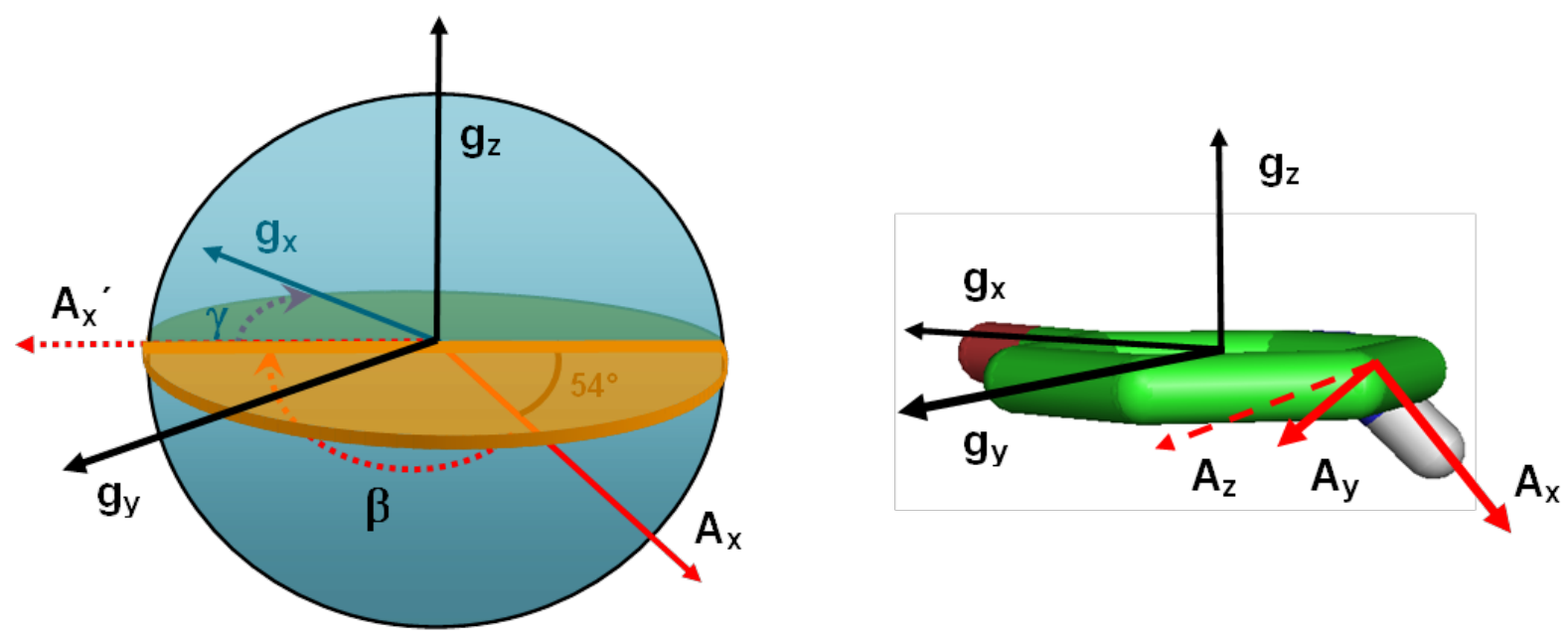

Figure 3-21. Euler rotation exemplified on the $\mathrm{N}-\mathrm{H}(2)$ bond. In case of $\mathrm{N}-\mathrm{H}(2)$, the first rotation occurs about $A_{y}$ and moves $A_{x}$ to $A_{x}^{\prime}$ (Euler angle $\beta$ ). The second rotation occurs about $A_{z}$ (Euler angle $\gamma$ ). See Table 3-1 for values of Euler angles for the $\mathrm{H} 2$ proton. For clarity, the translation of the other axes after each rotation is not shown (i.e. $A_{y}{ }^{\prime}$ and $A_{z}{ }^{\prime}$ ). The negative values of the angles are due to the requirement of counter-clockwise rotations in the Euler convention when moving the A-tensor out of the g-tensor frame (right side).

When we performed these rotations we realized immediately how sensitive the simulation was through the change of the $\beta$-angle within some degrees. The $\beta$-angle for this rotation results hereby in the out-of-plane position of the $\mathrm{N}-\mathrm{H}(2)$ bond which is $54^{\circ}$. Moreover the $\gamma$-angle is consistent with the form of a trigonal pyramide of the $\mathrm{NH}_{2}$-group when looking from top of the molecule.

In case of the $\mathrm{N}-\mathrm{H}(1)$ bond the Euler rotation resulted in an $\alpha$-angle which is in between $0^{\circ}$ and $40^{\circ}$ and an $\beta$-angle which is $50^{\circ}$. An $\alpha$-angle larger than $0^{\circ}$ represents a distortion of the trigonal pyramidal structure of the $\mathrm{NH}_{2}$ group whereas the $\beta$-angle places the $\mathrm{N}-\mathrm{H}(1)$ bond below the molecular plane like in the case of the N-H(2) bond (Figure 3-22). 

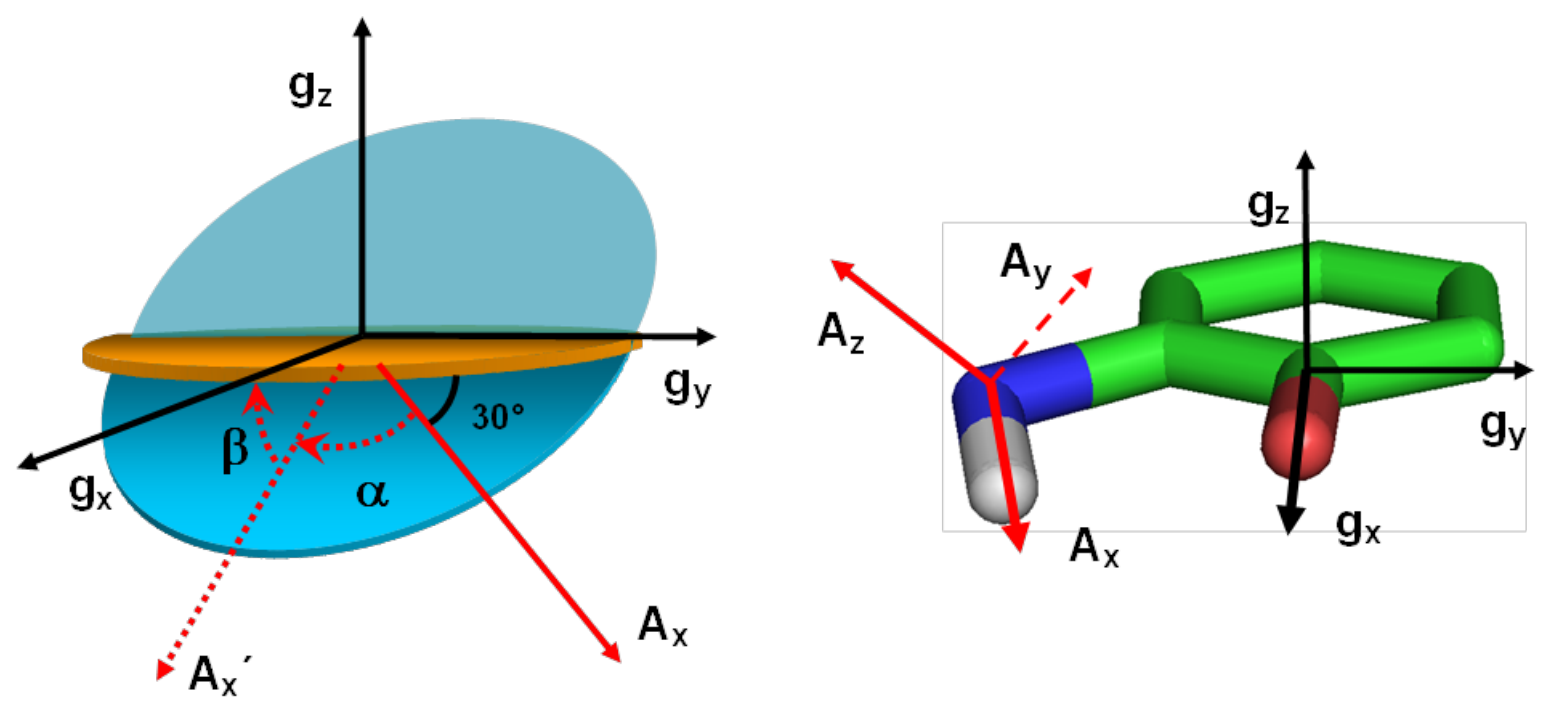

Figure 3-22. Euler rotation exemplified on the $\mathrm{N}-\mathrm{H}(1)$ bond. Two rotations are required to colinearize the $A$ - and g-tensor axes. The first rotation occurs about $A_{z}$ (Euler angle $\alpha$ ) and generates $A_{x}{ }^{\prime}$ co-linearizing $g_{y}$ and $A_{y}$. The second rotation occurs about $A_{y}{ }^{\prime}$ and corresponds to Euler angle $\beta$. This leads to co-linear $g_{x} / A_{x}$ and $g_{z} / A_{z}$ axes.

Again the simulation is very sensitive to slight changes of the $\beta$-angle. Due to uncertainty of the $\alpha$-angle the position of the $\mathrm{N}-\mathrm{H}(1)$ bond is less defined whereas the orientation of the $\mathrm{N}-\mathrm{H}(2)$ bond is well established within the amino-group. We also note that the hyperfine tensor of the $\mathrm{H} 1$ proton is substantially different, although the isotropic part is $\operatorname{similar}\left(\mathrm{A}_{\text {iso }}\right.$ $\left.(\mathrm{H}(2))=15 \mathrm{MHz} ; \mathrm{A}_{\text {iso }}(\mathrm{H}(1))=11 \mathrm{MHz}\right)$. This is likely caused by an additional dipolar interaction of $\mathrm{N}-\mathrm{H}(1)$ with the oxygen of the $\mathrm{C}-\mathrm{O}$ group. We conclude that the differences in the hyperfine tensors of the amino protons combined with the uncertain location of $\mathrm{N}-\mathrm{H}(1)$ strongly suggest the formation of an intramolecular hydrogen bond. Lastly the orientation of the $\mathrm{C}-\mathrm{N}$ bond is defined through the two Euler angles $\alpha$ and $\beta$ which are $60^{\circ}$ and $5^{\circ}$. The $5^{\circ}$ $\beta$-angle expresses the slight tilt of the $\mathrm{C}-\mathrm{N}$ bond whereas the $\alpha$-angle places the $\mathrm{C}-\mathrm{N}$ bond clockwise from the $\mathrm{C}-\mathrm{O}$ bond away. This is in agreement with an ortho-position of the $\mathrm{NH}_{2}{ }^{-}$ group in the 3-aminotyrosyl radical and supports the formation of an intramolecular hydrogen bond. As shown above in Table 3-1 the Euler angles are the same for each $\mathrm{NH}_{2} \mathrm{Y}^{\bullet}$ position within some degree of variation. The detailed structural characterization of the $\mathrm{NH}_{2}-$ group in the $\mathrm{NH}_{2} \mathrm{Y}^{\bullet}$ at position 730 leads therefore to the conclusion that the trigonal $\mathrm{NH}_{2}$-conformation together with an internal hydrogen bond formation is mandatory at each $\mathrm{NH}_{2} \mathrm{Y}$ location in the pathway. As a representative example of $\mathrm{NH}_{2} \mathrm{Y}^{\bullet}$ structures, the 
3-aminotyrosyl radical embedded in the protein at location 730 is presented in the Discussion section.

Finally the discrepancy of the $\mathrm{C}_{\beta}$-methylene proton couplings results in dihedral angles for the $\mathrm{C}_{\alpha}-\mathrm{C}_{\beta}$ bond with respect to the $\pi$-plane which differ from one $\mathrm{NH}_{2} \mathrm{Y}$ position to another one (Figure 3-23). The dihedral angle could be calculated through the usage of the McConnell equation $\left(A_{C \beta-H}=B_{1} \times \rho_{C 1} \times \cos ^{2} \Theta\right)$ which relates the isotropic $C_{\beta}$-methylene proton coupling to the electron spin density at the C1-carbon atom taking hereby the angle $\Theta$ between the $\mathrm{C}-\mathrm{H}$ bond and the $\mathrm{p}_{\mathrm{z}}$ orbital axis into account. $\mathrm{A} \Theta$ angle of $0^{\circ}$ leads to complete hyperconjugation between the $\mathrm{p}_{\mathrm{z}}$ orbital and the $\sigma$ orbital of the $\mathrm{C}-\mathrm{H}$ bond hereby leading to maximal overlap of these two orbitals and therefore to maximal hyperfine coupling value due to strongly induced electron spin polarization at the proton site. $\Theta$ angles which are bigger than $0^{\circ}$ decrease the hyperfine coupling.

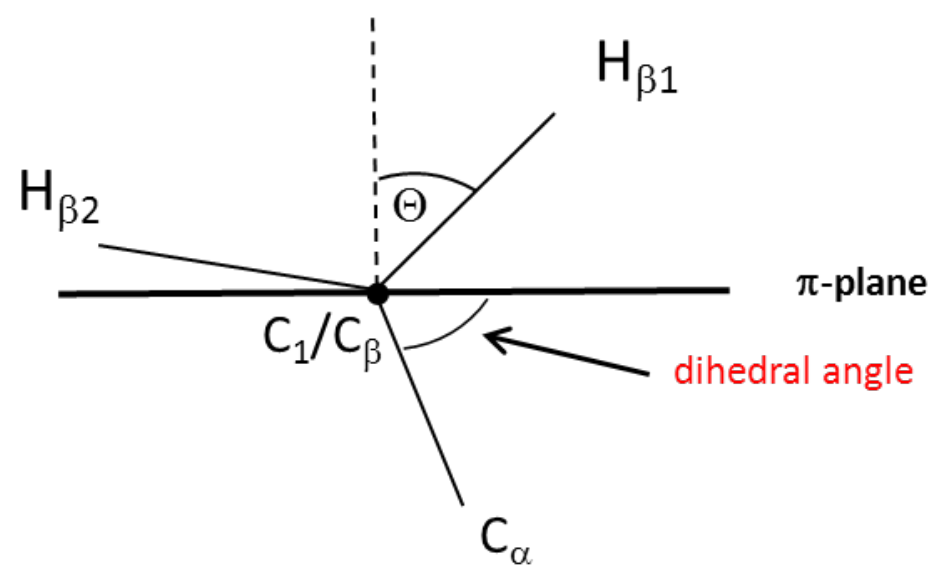

Figure 3-23. Definition of the dihedral angle and the angle $\Theta$. The three bonds form angles of $120^{\circ}$ when looking from the top. After calculation of $\Theta$ through the McConnell relation one can calculate easily the dihedral angle between the $\pi$-plane and the $C_{\alpha}-C_{\beta}$ bond of the protein chain which is $\Theta+\left(120^{\circ}-90^{\circ}\right)$. The dotted line represents the $p_{z}$ orbital axis at the $C 1$ position in the aromatic ring of the $\mathrm{NH}_{2} \mathrm{Y}^{*}$.

Using the isotropic value of $29.8 \mathrm{MHz}$ for the biggest hyperfine coupling value and the electron spin density of 0.2 at C1 (obtained from DFT calculations) together with the empirical constant $B_{1}$ of $162 \mathrm{MHz}$ one calculates a $\Theta$ value of $16^{\circ}$ which leads finally to a dihedral angle of $46^{\circ}$ for the $\mathrm{NH}_{2} \mathrm{Y}^{\bullet}$ at position 730 . The other two dihedral angles for 
position 731 and 356 are calculated to be $65^{\circ}$ and $55^{\circ}$ giving the isotropic hyperfine value of 22 and $26.8 \mathrm{MHz}$, respectively.

\subsection{Discussion}

Our experiments and analysis have allowed us to determine the g-values and the largest hyperfine tensors for all three $\mathrm{NH}_{2} \mathrm{Y}^{\bullet}$ on the radical propagation pathway. The g-values which are $g_{x}=2.0052, g_{y}=2.0042$ and $g_{z}=2.0022$ were first recorded using $180 \mathrm{GHz}$ pulsed EPR spectroscopy and have then been approved for $\mathrm{NH}_{2} \mathrm{Y}_{731}{ }^{\circ}$. They are slightly different for $\mathrm{NH}_{2} \mathrm{Y}_{356}{ }^{\circ}$. All $\mathrm{NH}_{2} \mathrm{Y}^{\circ} \mathrm{s}$ are in the range of phenoxy and ortho-substituted phenoxy radicals (23, 25-27). Comparing the $\mathrm{NH}_{2} \mathrm{Y}_{730^{\circ}}$ g-values with the g-values of $\operatorname{Tyr}_{122}{ }^{\bullet}\left(\mathrm{g}_{\mathrm{x}}=2.0091, \mathrm{~g}_{\mathrm{y}}=2.0046\right.$ and $g_{z}=2.0023$ ) reveals that $g_{y}$ and $g_{z}$ are similar and that $g_{x}$ is significantly suppressed. Below for comparison the two radicals $\operatorname{Tyr}_{122}{ }^{\bullet}$ and $\mathrm{NH}_{2} \mathrm{Y}_{730^{\circ}}$ are shown (Figure 3-24). Their orthorhombic tensors are different in shape. Especially the lower gx value of $\mathrm{NH}_{2} \mathrm{Y}_{730^{\circ}}$ (here being a representative for all $\mathrm{NH}_{2} \mathrm{Y}^{\bullet} \mathrm{s}$ ) changes the line shape significantly.
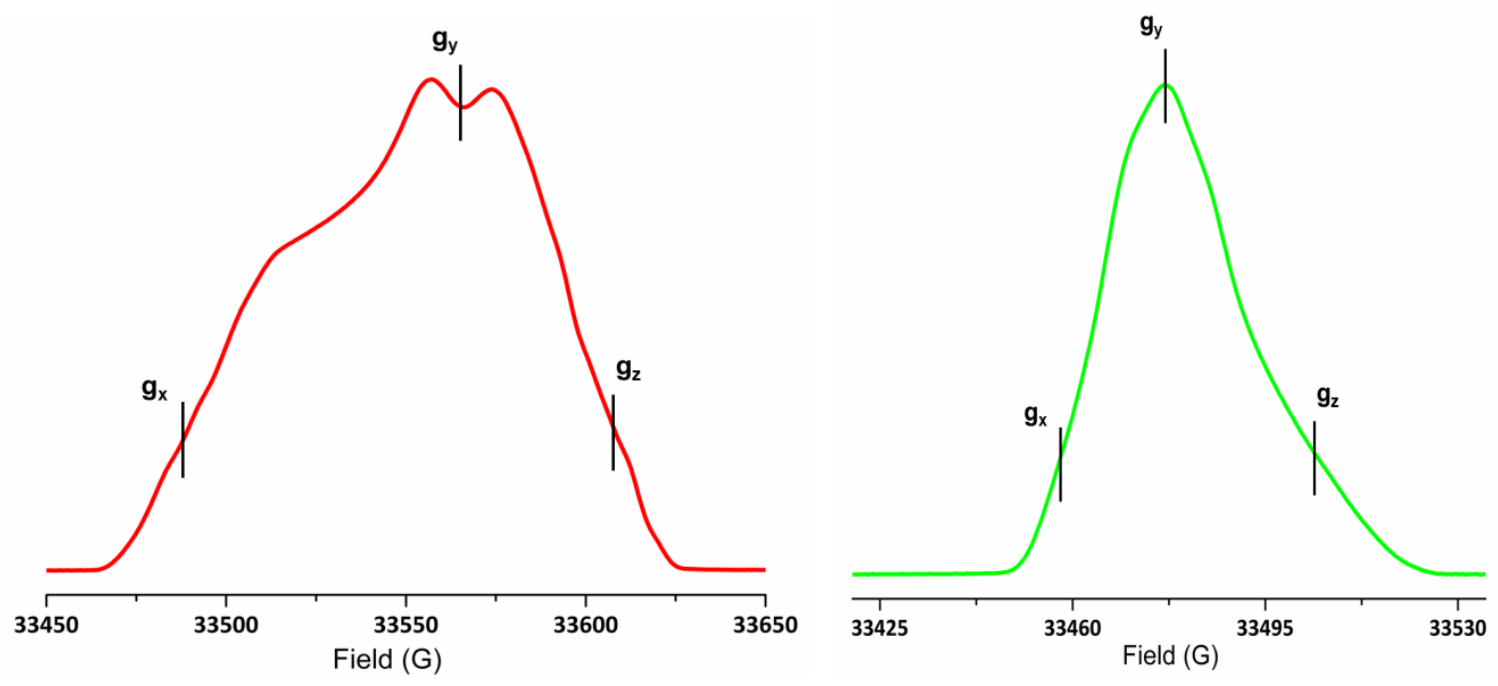

Figure 3-24. The two radicals present in the $\mathrm{NH}_{2} \mathrm{Y}_{730}-\alpha 2: \beta 2$ complex after incubation with substrate and effector (left $=\mathrm{Y}_{122}{ }^{\circ}$ and right $=\mathrm{NH}_{2} \mathrm{Y}_{730^{\circ}}$ ). The nature of both radicals is strongly influenced by the $g_{x}$ value. The g-values are indicated. 
A suppression of the $g_{x}$-value in case of $Y^{\circ}$ s has been found experimentally and validated by several theoretical studies to be associated with intermolecular hydrogen bonding to the phenoxy radical oxygen (28). Therefore in many $Y^{\bullet}$ studies of RNRs the $g_{x}$-value has been used as a diagnostic for the presence of external $\mathrm{H}$-bonds (see Chapter 1 Table 1-2 and references). Another mechanism for suppression of the $g_{x}$ value is ortho substitution of the phenoxy ring with a heteroatom such as $\mathrm{S}$ or $\mathrm{O}$, what is found for example in the thio-ether group like in galactose oxidase or in ortho-hydroxyphenoxy radicals like in one of our studies where a DOPA $^{\bullet}$ was generated at position 356 in the $\beta$-subunit (29). In both cases the $g_{x^{-}}$ value was suppressed to 2.0070 and 2.0056 , respectively. In case of the $\mathrm{NH}_{2} \mathrm{Y}^{\bullet}$ we have to take first the observed intramolecular $\mathrm{H}$-bond for the suppression of $\mathrm{g}_{\mathrm{x}}$ into account together with the ortho standing nitrogen heteroatom. Added to this complexity we show in the next chapter for the $\alpha-\mathrm{NH}_{2} \mathrm{Y}_{730^{\circ}}$ that it is surrounded by an intermolecular $\mathrm{H}$-bond network. On the other hand side due to the introduced heteroatom and the intrinsic intramolecular $\mathrm{H}$-bond it will be possible to distinguish a $\mathrm{NH}_{2} \mathrm{Y}^{\bullet}$ from other $\mathrm{Y}^{\bullet} \mathrm{s}$ within enzymes using high frequency EPR when $\mathrm{NH}_{2} \mathrm{Y}$ is inserted as a probe to trap radical intermediates.

Another important feature of the electronic structure which resulted from our study of the $\mathrm{NH}_{2} \mathrm{Y}_{730}{ }^{\circ}$ is the requirement for two amino protons in the simulation which consist of different hyperfine interactions. Together with the nitrogen hyperfine interactions a sp3 hybridized amino structure and not a sp2 hybridized results which would lead to a planar $\mathrm{NH}_{2}$-group. Taking also the $\mathrm{C}_{\alpha}-\mathrm{C}_{\beta}$-ring-plane dihedral angle into account $\mathrm{NH}_{2} \mathrm{Y}_{730}$ could be modeled into the $\alpha$ subunit with the obtained results from our EPR multifrequency study. Four conformations are theoretically possible in order to place the $\mathrm{NH}_{2} \mathrm{Y}_{730}{ }^{\circ}$ into the $\alpha$-subunit. However three of them lead to sterical clashes with surrounding amino acid residues and are inconsistent with X-ray crystallographic studies on $\mathrm{NH}_{2} \mathrm{Y}-\alpha 2$. In one case the $\mathrm{NH}_{2}$-group orientation does not result in any sterical clash and no $\mathrm{H}$-bond interactions between the $\mathrm{NH}_{2}$-protons with the nearby environment are expected besides the intramolecular $\mathrm{H}$-bond (Figure 3-25). Our observation that the $\mathrm{NH}_{2}$-group stays in the out-of-plane conformation are in agreement with earlier experimental and theoretical studies by Loth et al. on the ortho-aminophenoxyl radical. Their studies suggested an out-ofplane angle of $30^{\circ}$ for the $\mathrm{H} 1$ proton in the $\mathrm{N}-\mathrm{H} 1$ bond. The $\mathrm{H} 2$ in the $\mathrm{N}-\mathrm{H} 2$ bond stays in hereby in an out-of-plane angle of $15^{\circ}$. Nevertheless an intramolecular H-bond is formed $\mathbf{1 1 0} \mid \mathrm{P}$ a g e 
with the $\mathrm{H} 1$ proton in their studies and the conformation with a sp3 hybridized nitrogen consists of a lower energy with respect to the sp2 hybridized nitrogen in a planar conformation $(\Delta \mathrm{E}=0.5 \mathrm{kcal} / \mathrm{mol})$. The stabilization energy of one hydrogen-bond here can be up to $4-5 \mathrm{kcal} / \mathrm{mol}$.

Our simulations required further a $5^{\circ}$ tilt angle of the $\mathrm{C}-\mathrm{N}$ bond relative to the ring plane and Loth et al. found in their studies that the same tilt angle stabilizes the out-of-plane conformation. Their isotropic nitrogen hyperfine coupling which is depending on the solvent is $12.4\left(\mathrm{CHCl}_{2} / \mathrm{THF}\right)$ and $12.6 \mathrm{MHz}\left(\mathrm{CH}_{3} \mathrm{OH}\right)$ and is in very good agreement with our experimental one of $11.5 \mathrm{MHz}$. The hyperfine couplings of the $\mathrm{H} 2$ proton are 16.2 and 15.4 $\mathrm{MHz}$ and also in a very good agreement with our isotropic value of $15 \mathrm{MHz}$. However the isotropic $\mathrm{H} 1$ proton hyperfine coupling is $\sim 10 \mathrm{MHz}$ larger than our experimentally one. Their larger coupling value could result in a shorter intramolecular hydrogen-bond than we get out from our EPR analysis (see Figure 3-3). However Loth et al. did not report on the intramolecular hydrogen-bond length. Finally, recent DFT calculations predicted formation of an $\mathrm{H}$-bond in ortho-aminophenols in agreement with our results and those of Loth et al. (23).
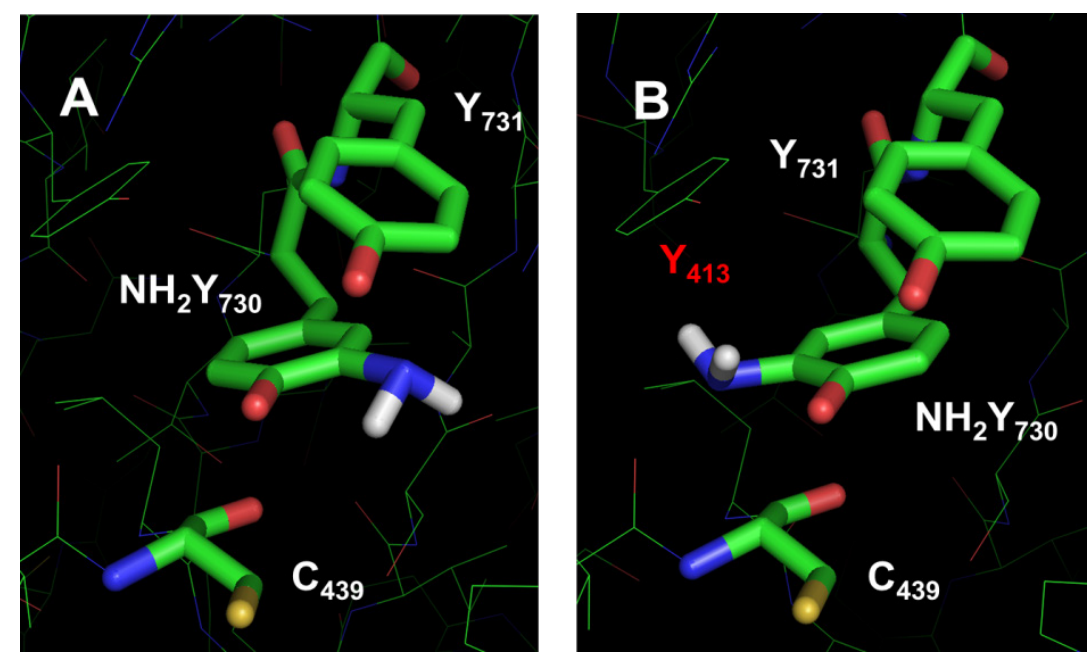

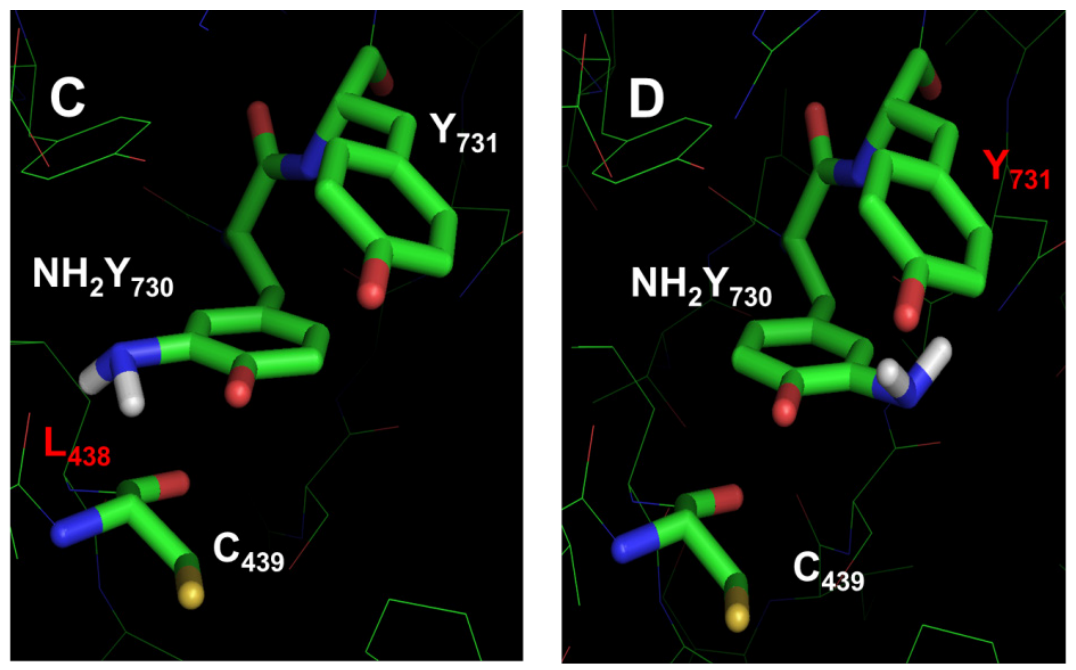

Figure 3-25. Models for the $\mathrm{Y}_{731}-\mathrm{NH}_{2} \mathrm{Y}_{730}-\mathrm{C}_{439}$ pathway in $\mathrm{NH}_{2} \mathrm{Y}_{730}-\alpha 2$. Models $\mathrm{B}, \mathrm{C}$ and $\mathrm{D}$ are consistent with the EPR data, but result in steric clashes within $\alpha 2$. In model B the $\mathrm{NH}_{2}$-group is to near to $Y_{413}$, in model $C$ to near to the backbone of the carbonyl of $L_{438}$ and in $D$ the amino group on the right side is too near to $\mathrm{Y}_{731}$. Furthermore $\mathrm{B}$ and $\mathrm{C}$ are inconsistent with the newest $\mathrm{X}$-ray structure of $\mathrm{NH}_{2} \mathrm{Y}-\alpha 2$ (3). Eliminating all these three possible models, results in only one model which is model $A$.

Our EPR analysis has provided constraints to model $\mathrm{NH}_{2} \mathrm{Y}$ at residue 730 in the $\alpha$-subunit in order to obtain insight into its position relative to the $Y_{731}$ and $C_{439}$ (Figure 3-26). The hyperfine coupling of the $\mathrm{C}_{\beta}$-methylene protons of the $\mathrm{NH}_{2} \mathrm{Y}^{\bullet}$ allowed calculation of the dihedral angle between $C_{\alpha}$ and $C_{\beta}$ of the protein chain and $p_{z}$ at $C_{1}$ being $46^{\circ}$. The angle is similar to the one determined for $Y_{730}$ in the crystal structure of wt $\alpha 2$ which is $36^{\circ}$. Thus, replacement of $\mathrm{Y}_{730}$ with $\mathrm{NH}_{2} \mathrm{Y}_{730}$ does not appear to significantly alter the orientation of this residue. In case of $\mathrm{NH}_{2} \mathrm{Y}_{731}$ the discrepancy of the dihedral angle obtained from the wt $\alpha 2$ crystal structure when compared to the EPR studies on the $\mathrm{NH}_{2} \mathrm{Y}_{731}{ }^{\bullet}$ is more pronounced. In wt $\alpha 2$ we measure a dihedral angle of $33^{\circ}$ but the angle obtained from our EPR studies is $65^{\circ}$. For $\mathrm{NH}_{2} \mathrm{Y}_{356^{\circ}}$ in the $\beta$-subunit we have no X-ray structural data available. 


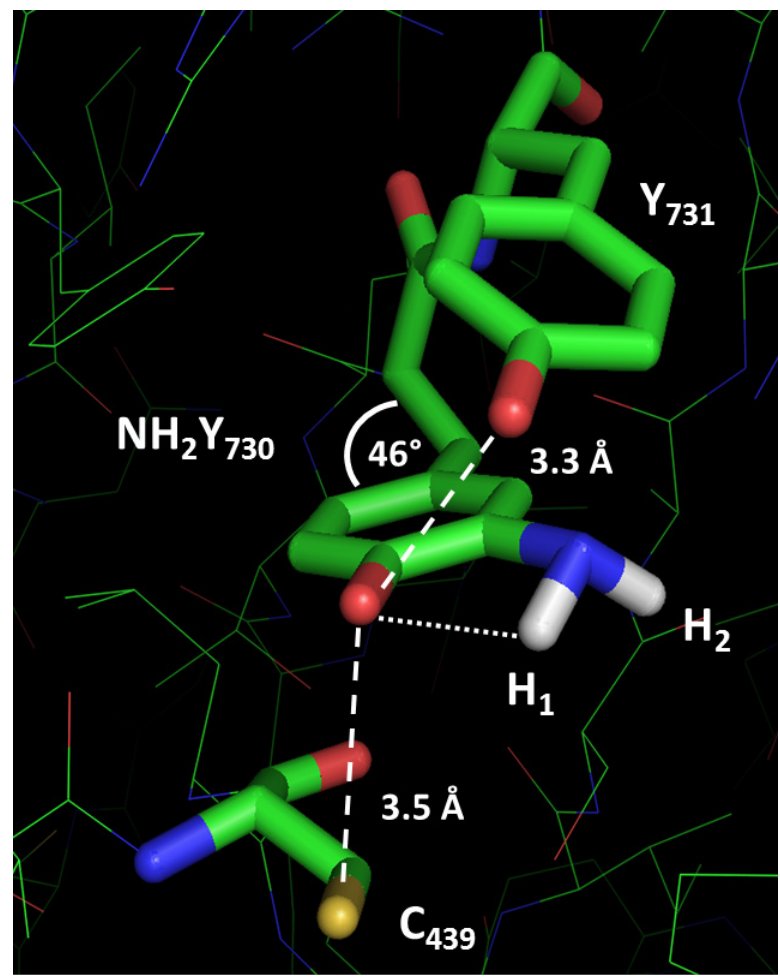

Figure 3-26. Model $A$ for the $\mathrm{Y}_{731}-\mathrm{NH}_{2} \mathrm{Y}_{730}-\mathrm{C}_{439}$ pathway in $\mathrm{NH}_{2} \mathrm{Y}_{730}-\alpha 2$. $\mathrm{NH}_{2} \mathrm{Y}$ has been modeled into residue 730 of $\alpha 2$ using the coordinates for wt crystal structure. The $C_{\alpha}-C_{\beta}$-ring-plane dihedral angle and the $\mathrm{NH}_{2}$-group conformation are based on results herein. The distance between the $\mathrm{H} 1$ from the $\mathrm{NH}_{2}$-group and the phenol $\mathrm{O}$ is indicated and the distance between the essential residues around $\mathrm{NH}_{2} \mathrm{Y}_{730}$ are illustrated as discussed in the text.

In conclusion the modeling together with the X-ray crystallographic studies suggested that the amino group does not affect the distances between the essential amino acids in the proposed PCET pathway indicating that the $\mathrm{NH}_{2} \mathrm{Y}$ probe can be used to measure the distance to the protons on $\mathrm{Y}_{731}(\mathrm{OH})$ and $\mathrm{C}_{439}(\mathrm{SH})$. In support of model $A$ it will be shown in the next chapter that the $\mathrm{C}-\mathrm{O}$ bonds of $\mathrm{Y}_{731}$ and $\mathrm{NH}_{2} \mathrm{Y}_{730}$ are staying nearly in one line to each other so that model D in Figure 3-25 could also be excluded. This was performed with high-field ENDOR experiments in $\mathrm{D}_{2} \mathrm{O}$ buffer which permitted the observation of intermolecular exchangeable protons together with their distances and orientations. The ENDOR experiment is hereby aggravated by the concomitant observation of the amino protons, which are also exchangeable. Therefore, the independent determination of the hyperfine tensors for the amino protons was a prerequisite for high-field ENDOR studies on $\mathrm{NH}_{2} \mathrm{Y}^{\bullet}$. 


\section{Chapter 4}

High-field ENDOR and DFT characterization of hydrogen-bond networks around $\alpha-\mathrm{NH}_{2} \mathrm{Y}_{730}{ }^{\circ}$ and $\alpha-\mathrm{NH}_{2} \mathrm{Y}_{731}$ in E.coli ribonucleotide reductase 


\subsection{Introduction}

In this chapter we concentrate on the elucidation of the hydrogen-bond network around $\mathrm{NH}_{2} \mathrm{Y}_{730}{ }^{\circ}$ and $\mathrm{NH}_{2} \mathrm{Y}_{731^{\circ}}$ in the $\alpha 2$ subunit of E.coli RNR through high frequency ENDOR spectroscopy in combination with DFT cluster calculations.

A hydrogen-bond network is seen as a prerequisite for the proposed $\mathrm{H}$-atom transfer or colinear PCET at both residue positions. As X-ray crystallography can not provide us with detailed information about proton locations, high frequency ENDOR can do this and gives direct evidence about the number, distances and orientations of $\mathrm{H}$-bonds in the active state of the E.coli RNR enzyme.

The structure of the $\alpha 2$ subunit was solved 1994 . Three years later a crystal structure with GDP and dTTP gave also detailed insight about bound substrate and effector at their specificity sites (PDB ID = 4R1R) (1). The effector dTTP is hereby positioned at the dimer interface at the end of a four helix bundle, whereas the substrate GDP is positioned near $\mathrm{CyS}_{439}$ with the C3' carbon being $3.4 \AA$ away from the sulphur atom. The O-S and O-O distances between $\mathrm{CyS}_{439}$ and $\mathrm{Tyr}_{731}$ as well as $\operatorname{Tyr}_{731}$ and $\operatorname{Tyr}_{730}$ are nearly the same, 3.4 and 3.3 Å (see Figure 1-6 in Chapter 1).

Recently also the crystal structure of $\mathrm{NH}_{2} \mathrm{Y}_{730}-\alpha 2$ (PDB ID = 2XO4) has been reported, showing that the $\mathrm{NH}_{2} \mathrm{Y}_{730}$ substitution has nearly no effect on the O-S and O-O inter atom distances (Figure 4-1). If the residues $\mathrm{Y}_{731}$ and $\mathrm{NH}_{2} \mathrm{Y}_{730}$ are positioned in a $\pi$-stacked conformation the maximum distance difference is $\pm 0.3 \AA$ in comparison to the wt structure. The first of three $\alpha$ monomers (the asymmetric unit is a trimer) is shown below with the $0-0$ and the O-S distance being 3.3 and $3.7 \AA$ respectively. 


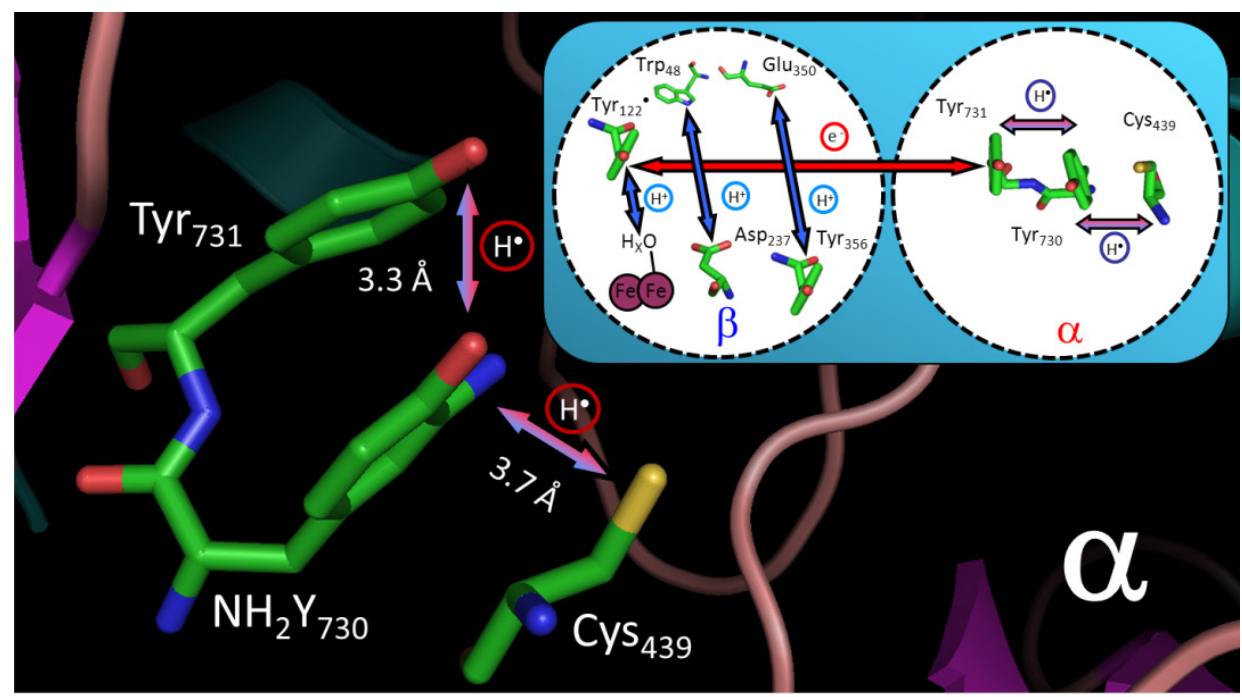

Figure 4-1. Crystal structure of the $\mathrm{NH}_{2} \mathrm{Y}_{730}-\alpha 2$ mutant of E.coli RNR (first of three $\alpha$ monomers) showing the residues $\mathrm{Y}_{731}, \mathrm{NH}_{2} \mathrm{Y}_{730}$ and $\mathrm{C}_{439}$ in the PCET pathway. The O-O distance and the O-S distance between the essential amino acid residues are indicated with the proposed co-linear PCET mechanism between the three residues. The inset shows the current model for the long-range ( $35 \AA$ ) ), reversible PCET starting at $Y_{122}$ in the $\beta$-subunit and reaching $C_{439}$ in the $\alpha$-subunit.

Quite interesting one of the monomers of the asymmetric unit shows $\mathrm{Tyr}_{731}$ in a flipped conformation away from $\mathrm{NH}_{2} \mathrm{Y}_{730}$. The reorientation of $\mathrm{Tyr}_{731}$ was not expected and it highlights the dynamic flexibility of residues at the $\alpha / \beta$ interface suggesting motions that may be possible upon $\alpha / \beta$ subunit interaction (see Figure $4-2$ ).

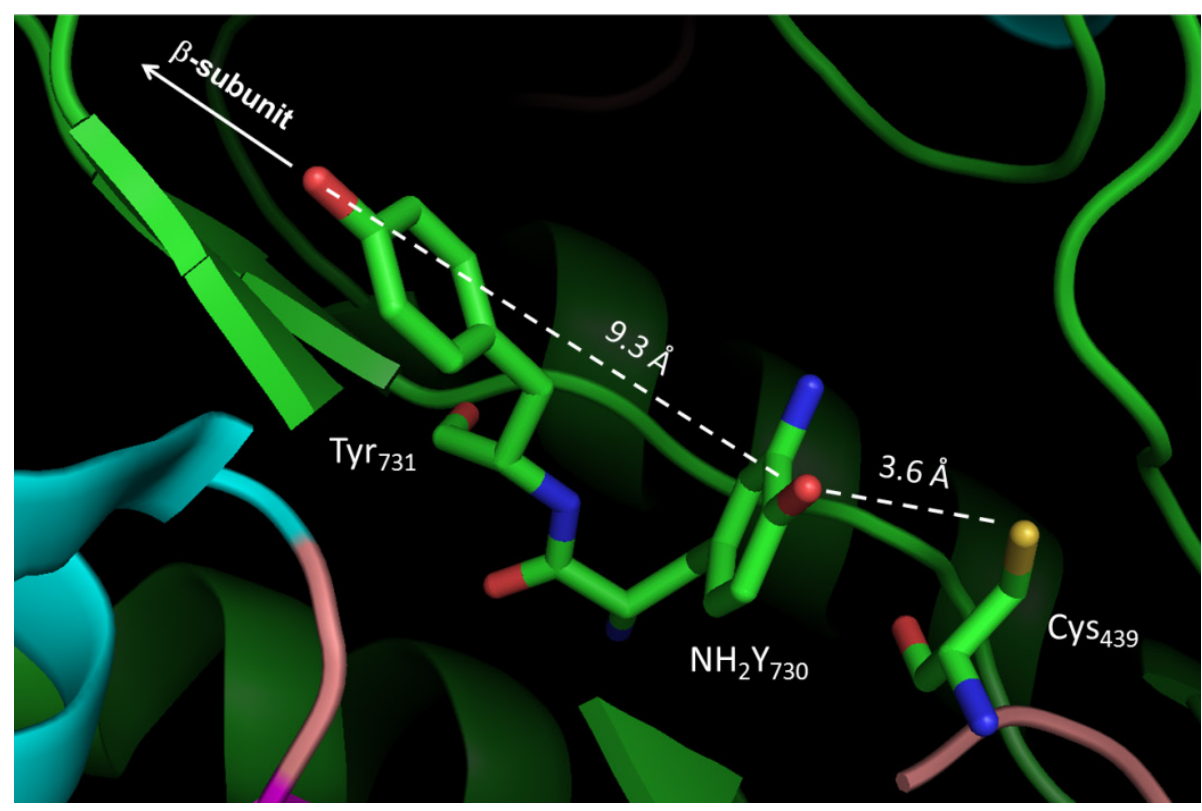


Figure 4-2. Crystal structure of the $\mathrm{NH}_{2} \mathrm{Y}_{730^{-}}-\alpha 2$ mutant of E.coli RNR in the flipped conformation (third of three $\alpha$ monomers) showing the residues $\mathrm{Y}_{731}, \mathrm{NH}_{2} \mathrm{Y}_{730}$ and $\mathrm{C}_{439}$ in the PCET pathway. The O-O distance in this conformation is $9.3 \AA$ but the O-S distance remains nearly unchanged within a difference of $0.1 \AA$ in comparison to the first monomer. In the flipped conformation $Y_{731}$ points into the direction of the $\beta$-subunit suggesting an interaction with $Y_{356}$.

$\mathrm{NH}_{2} \mathrm{Y}_{730}-\alpha$ was also co-incubated with a short peptide of the $\beta$-subunit like the wt structure. Although the crystallographic data of $\beta-Y_{356}$ are missing (the last residue is $Q_{360}$ ), the positioning of $\mathrm{Y}_{731}$ in the flipped conformation towards the peptide which is wrapped around the $\beta$-subunit is unambiguously visible (see Figure 4-3).

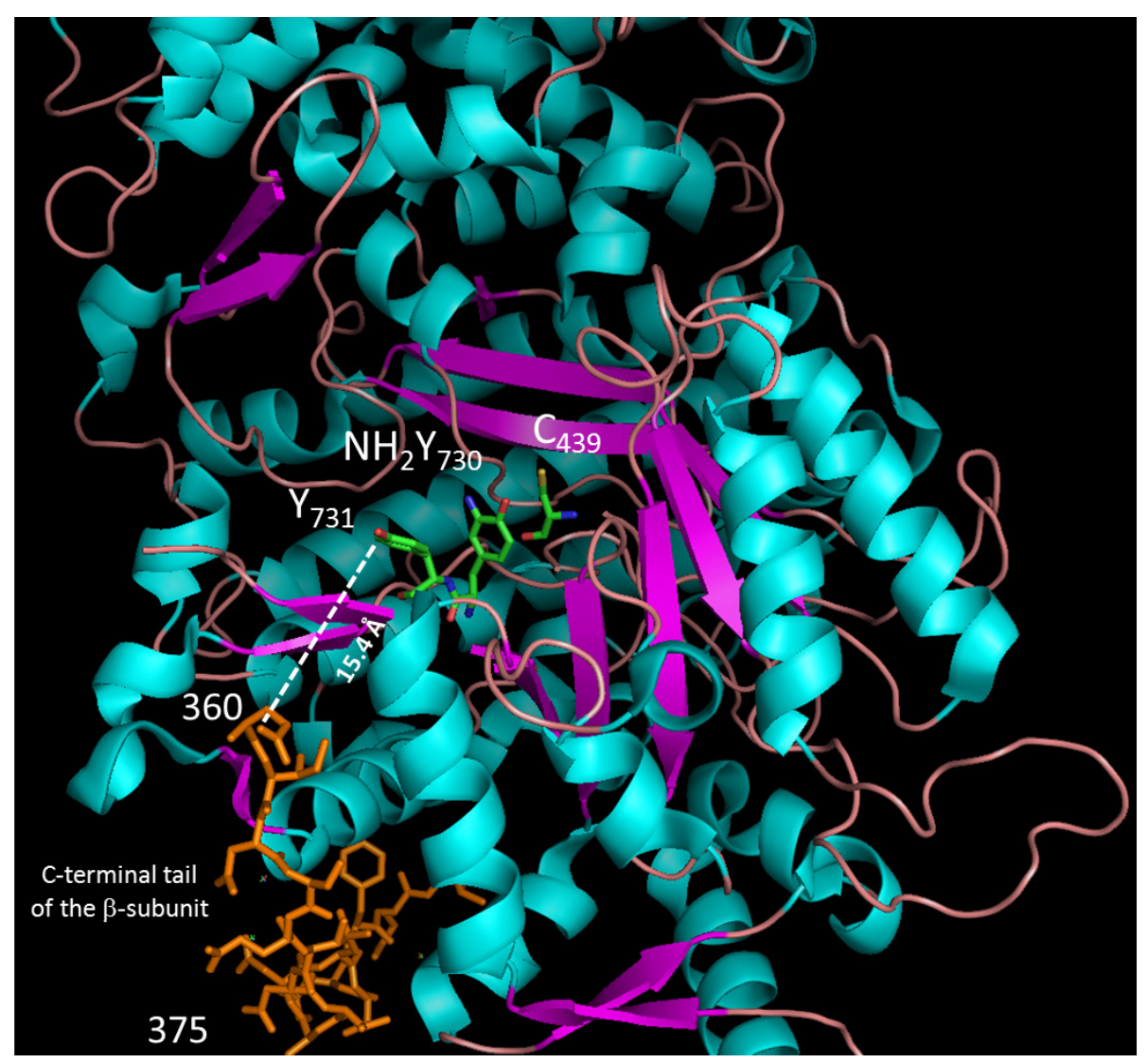

Figure 4-3. Crystal structure of the $\mathrm{NH}_{2} \mathrm{Y}_{730}-\alpha 2$ mutant of E.coli RNR in the flipped conformation (third of three $\alpha$ monomers) co-incubated with a 20-mer tail of the $\beta$-subunit (only the residues 360 to 375 are visible in the crystal structure). The distance from the C-O oxygen of $\alpha-Y_{731}$ to the oxygen of the side-chain of $\mathrm{Q}_{360}$ is indicated (the last visible residue). If we assume that the next four residues including $\beta-Y_{356}$ are positioned into the direction of $\alpha-Y_{731}$, then a C-O --- O-C distance in the range of 3-4 Å between $\beta-Y_{356}$ and $\alpha-Y_{731}$ can result. 
However EPR distance measurements (PELDOR) on hand quenched samples (second timescale) of incubated $\alpha 2-\mathrm{NH}_{2} \mathrm{Y}_{730 / 731}$ and $\beta 2$ determined a cross distance between $\mathrm{Y}_{122}{ }^{\bullet}$ in the $\beta$-subunit and $\mathrm{NH}_{2} \mathrm{Y}_{730^{\circ}}$ and $\mathrm{NH}_{2} \mathrm{Y}_{731}{ }^{\bullet}$ in the $\alpha$-subunit which results in a $\pi$-stacking conformation (2). This could further be confirmed through cross-distance measurements on the docking mode. Still the conformation of $Y_{731}$ could be different if one quenches the reaction on another timescale due to the visible flexibility of this residue in crystallographic studies.

The $\mathrm{NH}_{2} \mathrm{Y}_{731}-\alpha 2$ crystal structure (PDB ID = 2XO5) has also been reported and shows again that the residue at position 731 is flexible. In case of one monomer of the crystallized trimer, the $\mathrm{NH}_{2}$ group points to the left and for the other two monomers to the right direction when looking along the $\mathrm{C}-\mathrm{O}$ bond from the direction of the oxygen atom (see Figure 4-4). Therefore both $\mathrm{NH}_{2} \mathrm{Y}-\alpha 2$ mutant structures suggest high flexibility at position 731 but not at position 730.
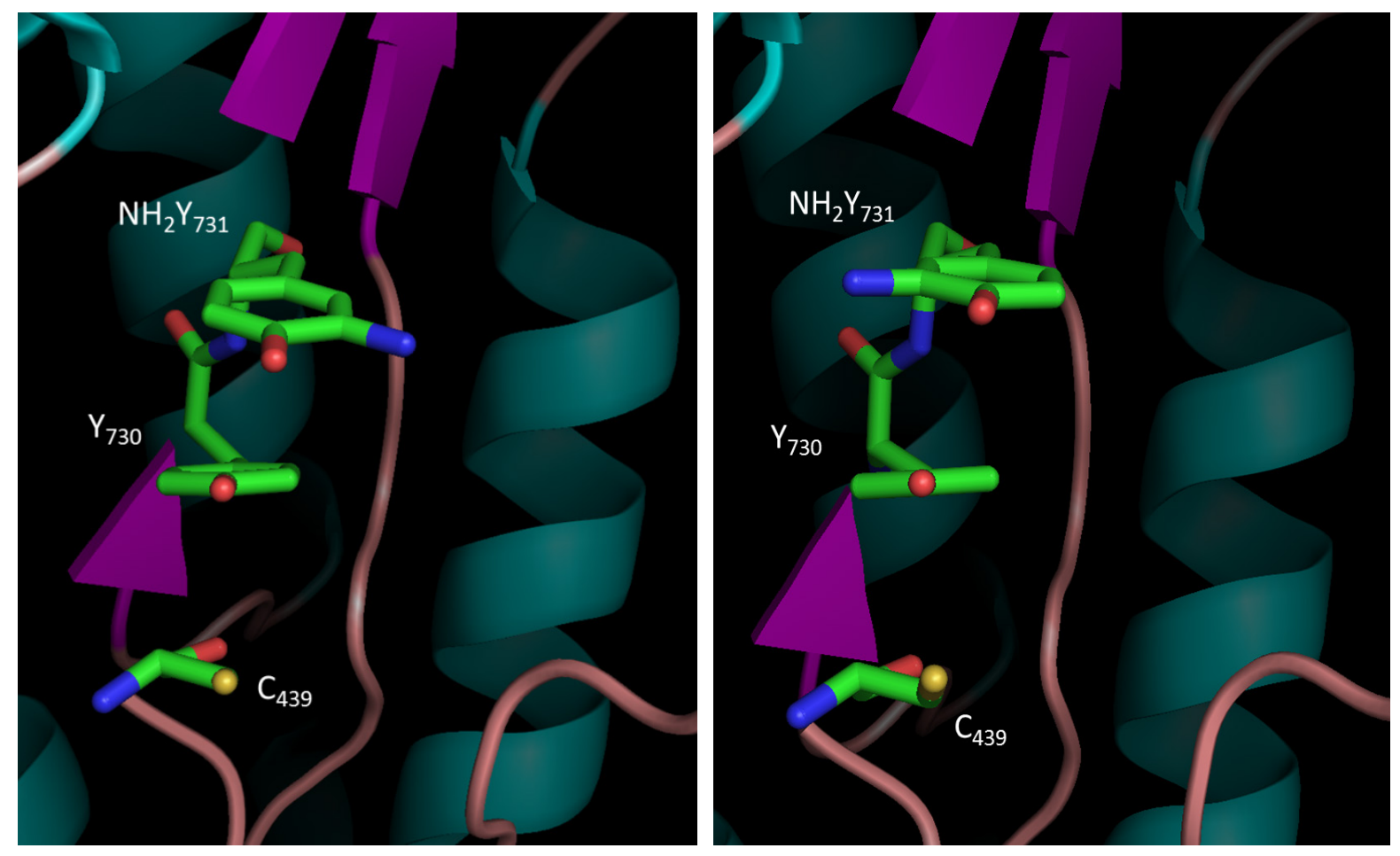

Figure 4-4. Crystal structure of the $\mathrm{NH}_{2} \mathrm{Y}_{731}-\alpha 2$ mutant of E.coli RNR showing two different conformations of $\mathrm{NH}_{2} \mathrm{Y}_{731}$ from the trimeric crystallophic unit, reflecting the high flexibility of this residue at position 731 . 
When incubating $\mathrm{NH}_{2} \mathrm{Y}_{730 / 731}-\alpha 2$ and $\beta 2$ in the presence of substrate and effector no activity of the enzyme was expected as $\mathrm{NH}_{2} \mathrm{Y}$ should function as a $190 \mathrm{mV}$ thermodynamic hole in the PCET pathway. However in comparison to the wt complex, 3-9 \% activity was detectable with turnover numbers for mutant $\alpha 2 \mathrm{~s}\left(\mathrm{k}_{\text {cat }}\right.$ of $\left.\sim 0.5 \mathrm{~s}^{-1}\right)$ which are ten times lower than in the wt complex ( $k_{\text {cat }}$ of $\left.2-10 s^{-1}\right)(3)$.

A conformational gating process which was reported earlier for wt E.coli RNR was also observed for $\mathrm{NH}_{2} \mathrm{Y}$-mutant E.coli RNRs with a slow phase of $\mathrm{k}_{\text {slow }}=1.5-5.0 \mathrm{~s}^{-1}$ (4). A second additional fast phase varied from $9-45 s^{-1}$ and demonstrated small dependence on the $\mathrm{NH}_{2} \mathrm{Y}$-position within the pathway. Both rate constants are believed to measure conformational changes rather than chemical events with $\mathrm{k}_{\text {slow }}$ reporting on conformational changes preceding catalysis (rate-determining step).

The amount of $\mathrm{NH}_{2} \mathrm{Y}^{\bullet}$ ranges from 30 to $40 \%$ of the $\mathrm{Y}_{122}{ }^{\bullet}$ that we start but only $20 \%$ of this $\mathrm{NH}_{2} \mathrm{Y}^{\bullet}$ initiates multiple nucleotide turnovers. If we are looking at this active state through high-field ENDOR spectroscopy we can gain insight about the hydrogen-bond interactions of the $\mathrm{NH}_{2} \mathrm{Y}^{\bullet}$ intermediate at each position of the PCET pathway which resembles the one in the wt complex as we expect that during activity the $\mathrm{NH}_{2} \mathrm{Y}-\alpha 2$ holo-protein is placed in a conformation similar to wt so that nucleotide turnover can occur.

Although we are looking at this stage likely on a composite of $\mathrm{NH}_{2} \mathrm{Y}^{\bullet}$ species, the $\mathrm{X}$-ray data shown above suggest that at position 730 the residue is fixed during radical propagation within the pathway. At position 731 the situation is different and we expect due to the flexibility of this residue in crystal structures a significantly different conformation when we freeze quench the reaction i.e. on the $\mu$ s timescale. However, in the second timescale we expect $\mathrm{NH}_{2} \mathrm{Y}_{731}$ to be mainly in a $\pi$ stacked conformation with $\mathrm{Y}_{730}$. The high-field ENDOR results reported herein for $\alpha-\mathrm{NH}_{2} \mathrm{Y}_{730}{ }^{\circ}$ and $\alpha-\mathrm{NH}_{2} \mathrm{Y}_{731}{ }^{\circ}$ are based on this conformation.

For the $\alpha$-subunit the $\mathrm{X}$-ray data of $\alpha-\mathrm{NH}_{2} \mathrm{Y}_{730}$ and $\alpha-\mathrm{NH}_{2} \mathrm{Y}_{731}$ suggested in case of most crystallographic units that the orientation of $\mathrm{NH}_{2} \mathrm{Y}_{730} / \mathrm{Y}_{730}$ and $\mathrm{Y}_{731} / \mathrm{NH}_{2} \mathrm{Y}_{730}$ relative to each other gives rise to $\pi$ - $\pi$-stacking and co-linear PCET or H-atom transfer. The proposal of colinear PCET is also supported by several experiments. Site-directed mutagenesis studies of $\alpha 2$ have shown that when $Y_{731}$ and $Y_{730}$ are mutated to phenylalanine the radical transfer is stopped due to a missing hydrogen bond (inactive mutant) (5). Furthermore a pure electron transfer among residues $\mathrm{Y}_{731}, \mathrm{Y}_{730}$ and $\mathrm{C}_{439}$ could be excluded through experiments with engineered photo-RNRs and diverse peptide-attached photooxidants $(6,7)$. 
We have characterized $\mathrm{NH}_{2} \mathrm{Y}_{730^{\circ}}$ and $\mathrm{NH}_{2} \mathrm{Y}_{731}{ }^{\circ}$ in the $\alpha$-subunit through multifrequency EPR spectroscopy and revealed unprecedented g-values $\left(g_{x}=2.0052 ; g_{y}=2.0042\right.$ and $\left.g_{z}=2.0022\right)$ in comparison to known g-values for $\mathrm{Ys}^{\bullet}$ which are either $\mathrm{H}$-bonded or not. From the very low $g_{x}$-value it was suggested that characteristic electrostatic and $\mathrm{H}$-bond interactions are present around the radical (see Chapter 3 ). The previous study prompted us to examine in more detail the structure around $\mathrm{NH}_{2} \mathrm{Y}_{730}{ }^{\circ}$ and find evidence for the proposed intermolecular hydrogen-bond network to the next essential amino acids $Y_{731}$ and $C_{439}$ on the PCET pathway. For this purpose we have performed high-frequency ENDOR spectroscopy in order to detect the hyperfine coupling to deuterons which replaced the positions of protons after $\mathrm{D}_{2} \mathrm{O}$ buffer exchange. Specifically the method at high fields permits to separate the resonances of the deuterons from ${ }^{1} \mathrm{H}$ resonances of non-exchangeable protons (i.e. ring protons) or other low- $\gamma$ nuclei such as ${ }^{13} \mathrm{C}$ or ${ }^{14} \mathrm{~N}$ of the $\mathrm{NH}_{2} \mathrm{Y}_{730}$ (i.e. from the $\mathrm{NH}_{2}$-group or the ring carbons). Additionally the high-field/high-frequency approach allows also for so called orientation-selective ENDOR experiments. Only a few molecular orientations are excited with this approach. If however one records several of such ENDOR spectra across the EPR line by using specific pulse lengths it allows not only the detection of all hyperfine tensor orientations at once (like in X-band for organic radicals) but permits the reconstruction of different hyperfine orientations in the molecular or g-tensor frame. Simulating all orientation selective ENDOR traces in one run allows the determination of hyperfine and quadrupole parameters (in case of ${ }^{2} \mathrm{H}$ ) together with Euler angles which result in a geometrical orientation of the A- and Q-tensor with respect to the g-tensor frame.

Nevertheless, the interpretation of the EPR data in terms of a structure requires also the use of sophisticated quantum chemical calculations. There are essentially two approaches to model enzymes (8). In one approach, a small quantum chemical (QM) model is surrounded by a molecular mechanics (MM) part which usually describes the rest of the enzyme. This QM/MM methodology using DFT has not so far been used to study PCET processes in enzymes to any significant degree. Instead, the most common approach in this context has been to use the DFT cluster model, where all atoms are described quantum mechanically. The models have been gradually increased with time and can now contain up to 250 atoms. To model the rest of the enzyme, a simple dielectric cavity with fixed dielectric constant (usually $\varepsilon=4.0$ ) has most often been used. 
Below in the results section it is shown that the combination of high-field ENDOR and DFT methods allowed the determination of the number, distances and orientations of exchangeable protons around the $\alpha-\mathrm{NH}_{2} \mathrm{Y}_{730}{ }^{\bullet}$ and $\alpha-\mathrm{NH}_{2} \mathrm{Y}_{731}{ }^{\bullet}$ intermediate. The calculated EPR parameters were important for the interpretation of all orientation selective ENDOR spectra and could approve the experimental g-values through the involvement of a hydrogen-bond network around $\alpha-\mathrm{NH}_{2} \mathrm{Y}_{730}{ }^{\circ}$ and $\alpha-\mathrm{NH}_{2} \mathrm{Y}_{731}{ }^{\circ}$.

Our study revealed in case of $\alpha-\mathrm{NH}_{2} \mathrm{Y}_{730}{ }^{\circ}$ that two hydrogen-bonds are directed towards the PCET pathway residues $\operatorname{Tyr}_{731}$ and $\mathrm{Cys}_{439}$ and additionally it was surprisingly revealed that a water molecule is placed $2.6 \AA$ away from the $\mathrm{NH}_{2} \mathrm{Y}_{730} 0^{\circ}$ intermediate $\left(\mathrm{O}^{\circ}\right.$-D distance). This water molecule has not been considered to date in a possible PCET mechanism within $\alpha 2$ and could have substantial impact on the $\mathrm{H}$-atom transfer rates between the three essential residues in the PCET pathway of $\alpha 2$.

First orientation selective ENDOR measurements on $\alpha-\mathrm{NH}_{2} \mathrm{Y}_{731}{ }^{\bullet}$ (quenched at the second timescale) revealed through ENDOR simulations three different deuterons, from which two of them are in the same hydrogen-bond distance range like in case of $\alpha-\mathrm{NH}_{2} \mathrm{Y}_{730}$ and point also into the direction of PCET pathway residues either in the $\alpha$ - or in the $\beta$-subunit. The third deuteron originates most probably from a water molecule $3.5 \AA$ away from the $\mathrm{NH}_{2} \mathrm{Y}_{731}{ }^{\circ}$ intermediate $\left(\mathrm{O}^{\circ}\right.$-D distance). EPR parameters obtained from DFT calculations on $\alpha$ $\mathrm{NH}_{2} \mathrm{Y}_{730^{\circ}}$ could hereby successfully be used to approve the experimentally detected hydrogen-bonds. A calculation for the detected far deuteron has still to be performed. 


\subsection{Results}

High-field ENDOR spectroscopy at $94 \mathrm{GHz}$ of $\alpha-\mathrm{NH}_{2} \mathrm{Y}_{730^{\circ}}$ and $\alpha-\mathrm{NH}_{2} \mathrm{Y}_{731}{ }^{\circ}$ in deuterated buffer

The ENDOR spectra were recorded with the Mims pulse sequence which is preferably used for the detection of deuterons in a radius of up to $5 \AA$ around a paramagnetic center. After $\mathrm{D}_{2} \mathrm{O}$ buffer exchange all protons of functional groups like $\mathrm{O}-\mathrm{H}, \mathrm{S}-\mathrm{H}, \mathrm{NH}_{2}$ as well as protons from the backbone amid groups get replaced through deuterons. The two spectra are shown below composed by a complex hyperfine line pattern which consist of at least more than one deuterium resonance (Figure 4-2 and 4-3). Each deuterium contributes hereby to the spectrum with a line pattern characterized by a hyperfine and a quadrupol splitting. For deuterons with pure dipole-dipole interaction a quartet pattern is visible (see also Figure 1-33 Chapter 1).

The observation of a complex ${ }^{2} \mathrm{H}$ ENDOR signal with a spectral width of up to $4 \mathrm{MHz}$ was expected in case of both samples due to the first examination of proton resonances of the $\mathrm{NH}_{2}$-group of the $\mathrm{NH}_{2} \mathrm{Y}_{730}$ probe by multifrequency EPR spectroscopy. The largest hyperfine coupling value of a proton ( $\left|A_{z z}\right|=27.6 \mathrm{MHz}$ for $\mathrm{H} 2$ ) corresponds in deuteron frequency to 4.2 $\mathrm{MHz}$ and is in very good agreement with the spectral width of both ${ }^{2} \mathrm{H}$ ENDOR spectra. Several other overlapping signals are also visible but most prominent are the strong features on top of the resonance shoulders at around $\pm 0.6 \mathrm{MHz}$ for $\mathrm{ND}_{2} \mathrm{Y}_{730}$ and $\pm 0.8 \mathrm{MHz}$ for $\mathrm{ND}_{2} \mathrm{Y}_{731^{\circ}}$. A well defined Pake pattern of a weakly coupled deuteron around the ${ }^{2} \mathrm{H}$ Larmor frequency is visible only in case of the $\mathrm{ND}_{2} \mathrm{Y}_{730^{\circ}}$ probe and the strong peaks at \pm 0.6 and \pm 0.8 $\mathrm{MHz}$ are expected to originate from strongly coupled hydrogen bonds (9). 


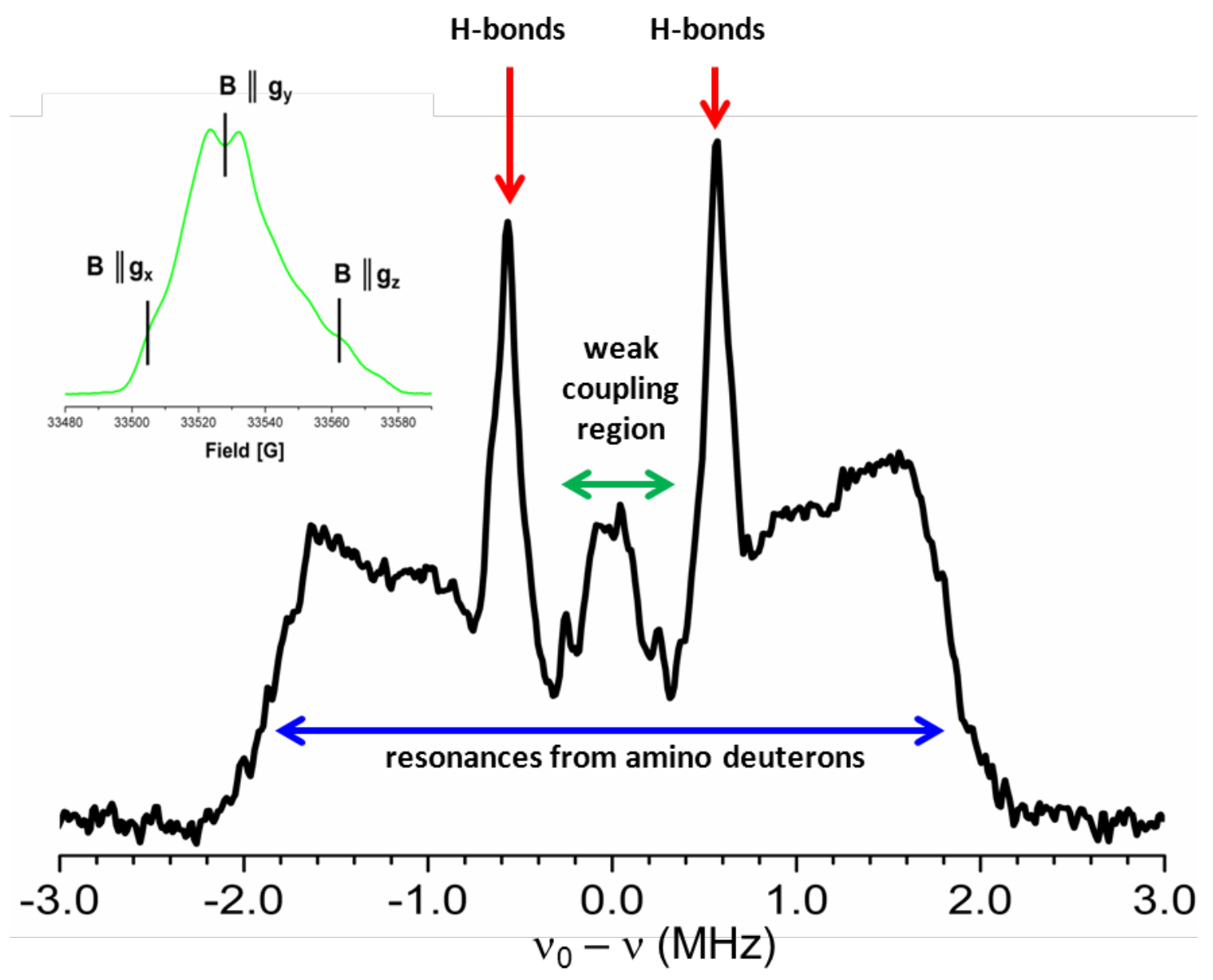

Figure 4-5. $94 \mathrm{GHz}^{2} \mathrm{H}$ Mims ENDOR spectrum of $\mathrm{NH}_{2} \mathrm{Y}_{730}{ }^{\bullet}$ in $\mathrm{D}_{2} \mathrm{O}$ assay buffer generated after reaction of $\mathrm{NH}_{2} \mathrm{Y}_{730}$ with CDP/ATP and freeze quenching with liquid $\mathrm{N}_{2}$ after $30 \mathrm{~s}$. The spectrum was recorded at $B \| g_{y}$ (see absorptive EPR spectrum in the inset left of $\mathrm{NH}_{2} \mathrm{Y}_{730} 0^{\circ}$ in $\mathrm{D}_{2} \mathrm{O}$ buffer). Three different resonant regions are indicated with arrows and are discussed in the text. Experimental conditions (for field sweep): $\mathrm{T}=70 \mathrm{~K}, \mathrm{MW}(\pi / 2)=32 \mathrm{~ns}, \tau=260 \mathrm{~ns}, \mathrm{MW}(\pi)=64 \mathrm{~ns}$, repetition time $=3 \mathrm{~ms}$, shots $/$ point $=50$, scans $=700$. Experimental conditions (for ENDOR): $\mathrm{T}=10 \mathrm{~K}, \mathrm{MW}$ pulse $(\pi / 2)=20 \mathrm{~ns}$, $\tau=200 \mathrm{~ns}, \mathrm{RF}$ pulse $=40 \mu \mathrm{s}$, repetition time $=150 \mathrm{~ms}, 2660$ scans, $55 \mathrm{~h}$ acquisition time. 


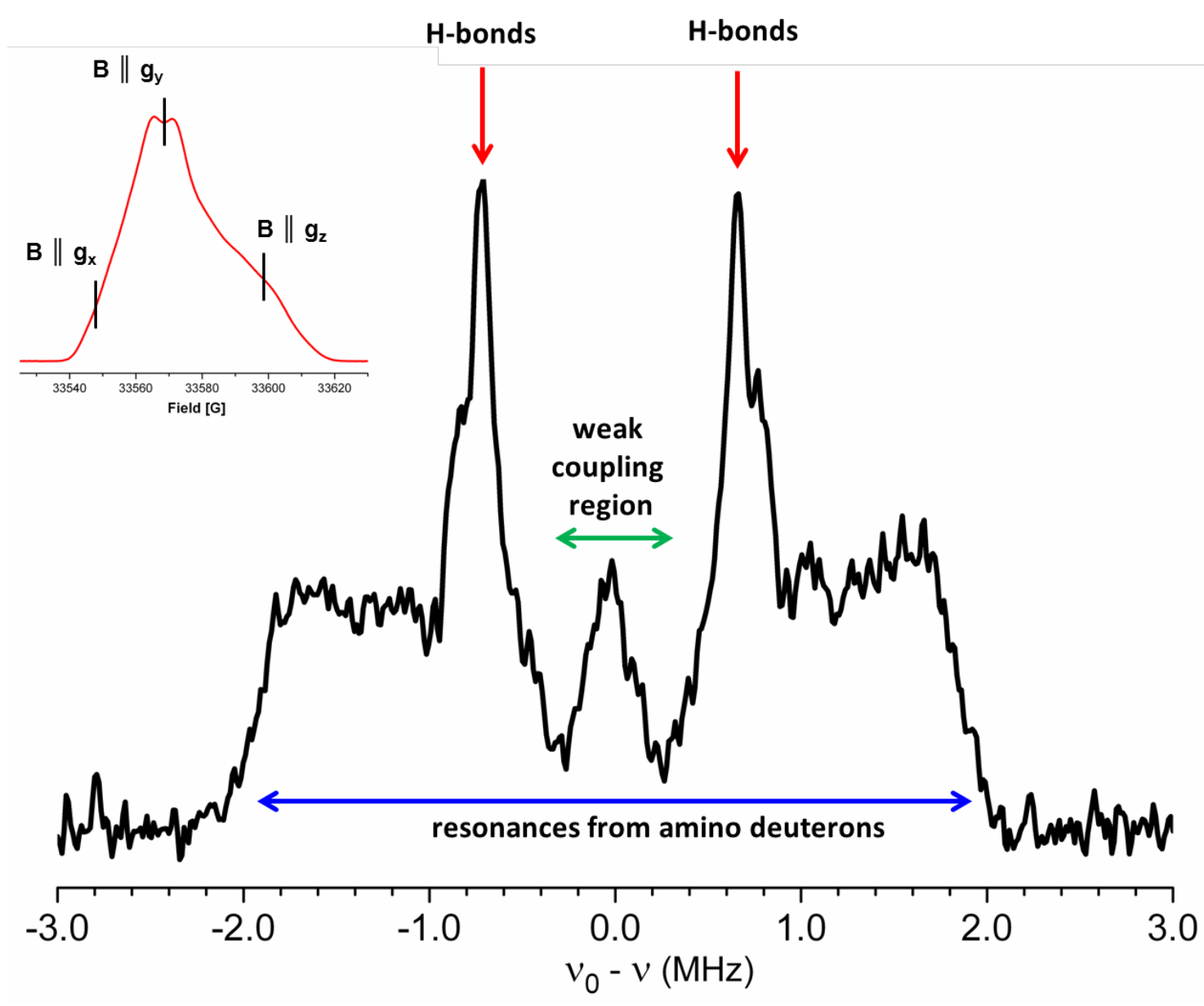

Figure 4-6. $94 \mathrm{GHz}^{2} \mathrm{H}$ Mims ENDOR spectrum of $\mathrm{NH}_{2} \mathrm{Y}_{731}$ in $\mathrm{D}_{2} \mathrm{O}$ assay buffer generated after reaction of $\mathrm{NH}_{2} \mathrm{Y}_{731}$ with CDP/ATP and freeze quenching with liquid $\mathrm{N}_{2}$ after $30 \mathrm{~s}$. The spectrum was recorded at $B \| g_{y}$ (see absorptive EPR spectrum in the inset left of $\mathrm{NH}_{2} \mathrm{Y}_{731}{ }^{\bullet}$ in $\mathrm{D}_{2} \mathrm{O}$ buffer). Three different resonant regions are indicated with arrows which are discussed in the text. Experimental conditions (for field sweep): $\mathrm{T}=70 \mathrm{~K}, \mathrm{MW}(\pi / 2)=16 \mathrm{~ns}, \tau=260 \mathrm{~ns}, \mathrm{MW}(\pi)=32 \mathrm{~ns}$, repetition time $=5 \mathrm{~ms}$, shots $/$ point $=50$, scans $=1070$. Experimental conditions (for ENDOR): $\mathrm{T}=10 \mathrm{~K}, \mathrm{MW}$ pulse $(\pi / 2)=20$ $\mathrm{ns}, \tau=200 \mathrm{~ns}, \mathrm{RF}$ pulse $=40 \mu \mathrm{s}$, repetition time $=150 \mathrm{~ms}, 2660$ scans, $55 \mathrm{~h}$ acquisition time.

For comparison the two $94 \mathrm{GHz}^{2} \mathrm{H}$ Mims ENDOR spectra have been overlayed (Figure 4-4). The spectral width is in both cases the same as well as a step in the resonance shoulder which results from an overlap of resonances coming from deuterons with medium hyperfine coupling of the $\mathrm{ND}_{2}$ group $( \pm 1.4 \mathrm{MHz}$ ) and from the hyperfine tensor of the external deuterons. The spectra however differ in the low coupling region and the coupling to external hydrogens is in case of $\mathrm{ND}_{2} \mathrm{Y}_{731}{ }^{\circ}$ stronger when compared with $\mathrm{ND}_{2} \mathrm{Y}_{730}{ }^{\circ}$ (an increase of $0.2 \mathrm{MHz}$ ). 


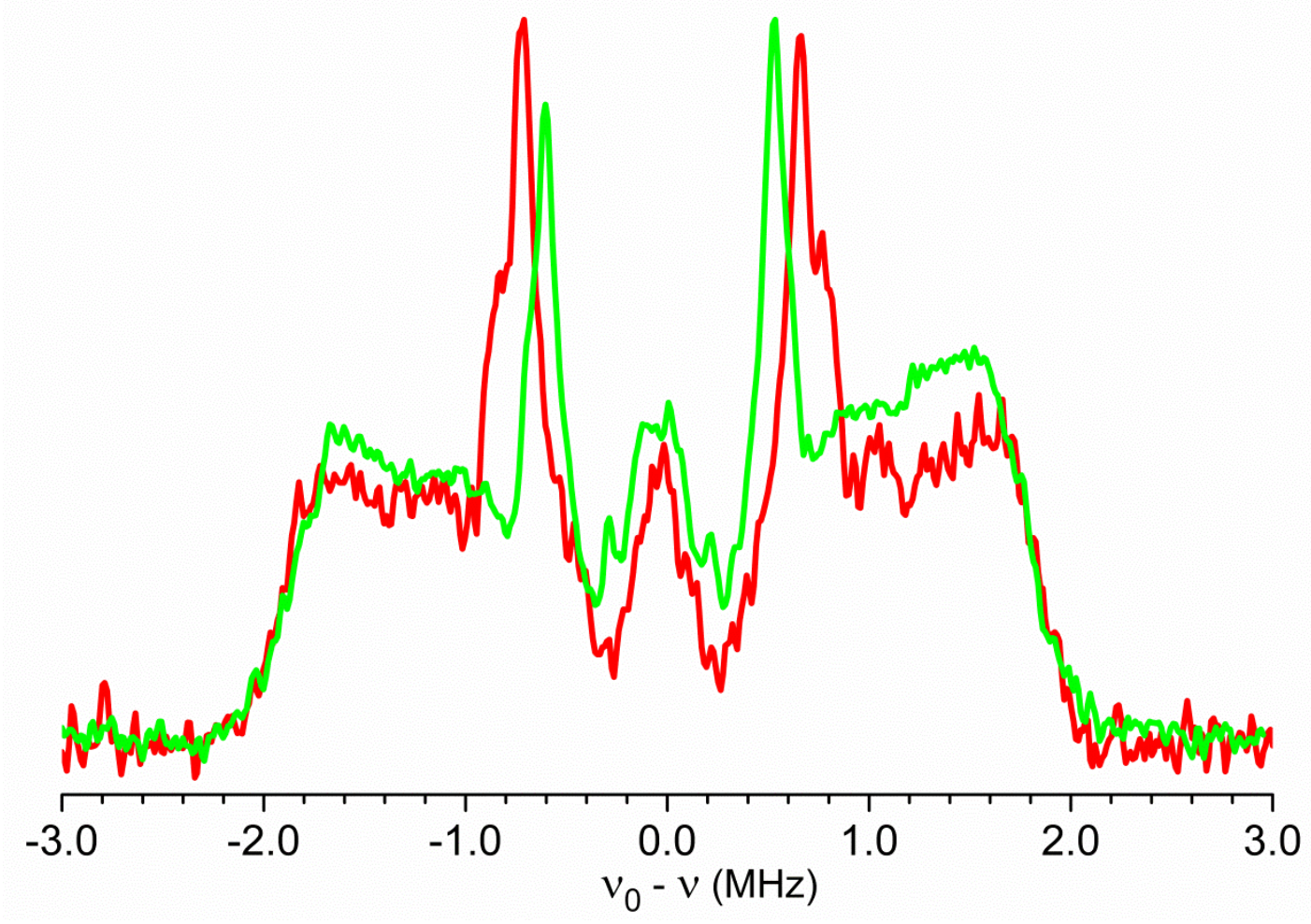

Figure 4-7. Comparison of both $\mathrm{NH}_{2} \mathrm{YS}^{\bullet}$ (green spectrum $=\mathrm{NH}_{2} \mathrm{Y}_{730^{\circ}}$, red spectrum $=\mathrm{NH}_{2} \mathrm{Y}_{731}{ }^{\circ}$ ) in $\mathrm{D}_{2} \mathrm{O}$ assay buffer which were recorded under the same experimental ENDOR conditions. Differences appear in the low coupling region and for the external H-bonds. However the spectral width is the same when recording the spectra at $\mathrm{B} \| \mathrm{g}_{\mathrm{y}}$.

As a descriptive example we have also recorded $N_{2} Y_{730^{\circ}}$ with a $\tau$-value of 360 ns. The spectrum is overlayed with the previously recorded one with $\tau=200 \mathrm{~ns}$ (Figure 4-5). What is immediately visible is that the spectrum recorded with the longer $\tau$ consists no spectral information in the region around $\pm 1.2 \mathrm{MHz}$. It is the first blind spot for this $\tau$ value as demonstrated also in Figure 1-31 in the introduction. However the width of the spectrum remains the same although the edges of the spectrum are lower in intensity when compared with the spectra with $\tau=200 \mathrm{~ns}$. On the other hand the measurement with the shorter $\tau$ places the first blind spot outside the ENDOR spectral region but suppresses the ENDOR signal around the nuclear Zeeman frequency. For an exact analysis of the three different spectral regions (see Figure 4-2 and 4-3) three different $\tau$-values in the orientation selective ENDOR experiments of $\mathrm{ND}_{2} \mathrm{Y}_{730}{ }^{\circ}$ and $\mathrm{ND}_{2} \mathrm{Y}_{731}{ }^{\circ}$ have been used as demonstrated in the next sections. 


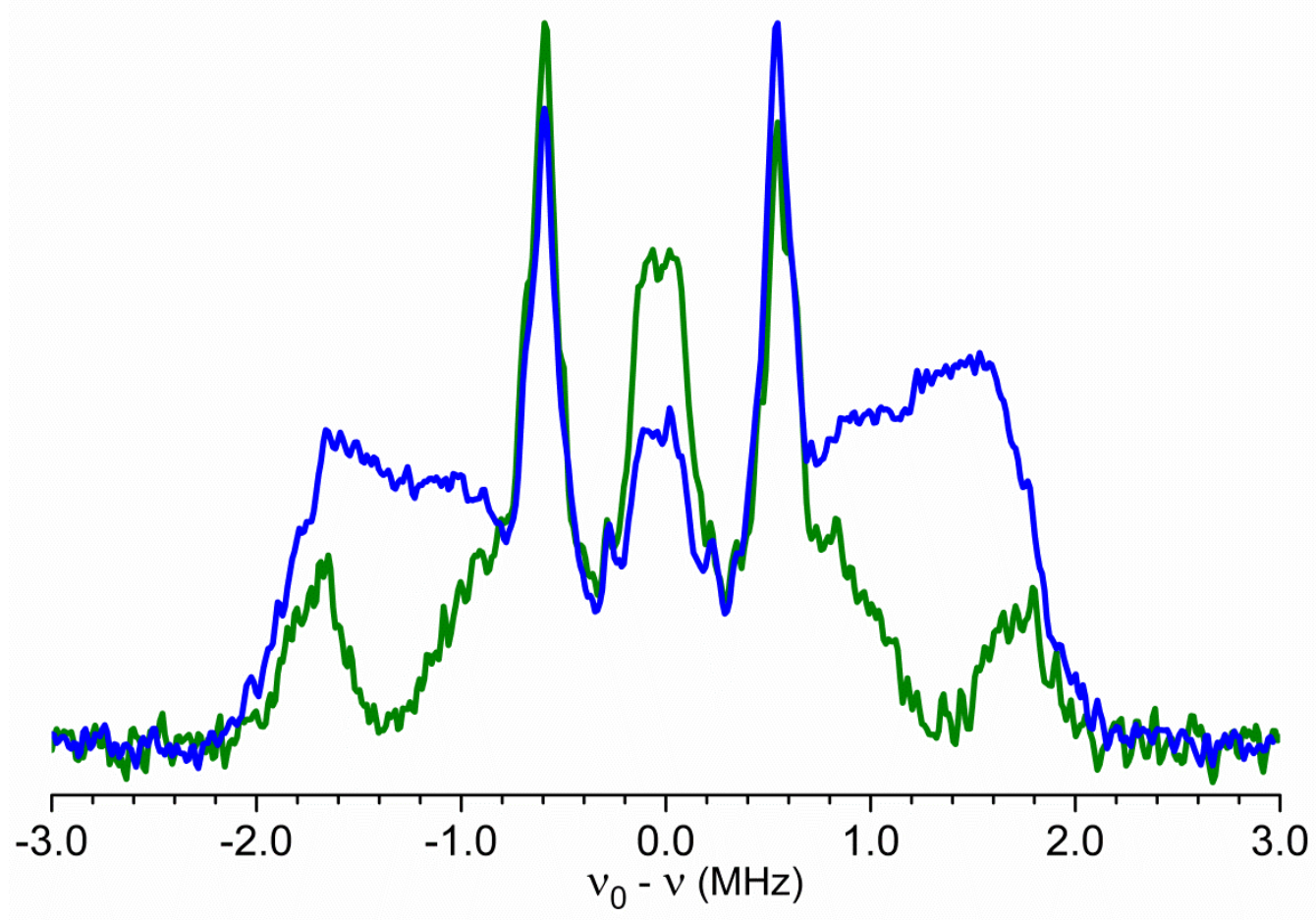

Figure 4-8. Comparison of two $\mathrm{ND}_{2} \mathrm{Y}_{730}{ }^{\circ}$ ENDOR spectra measured with two different $\tau$-values. Experimental conditions (for blue spectrum): $\mathrm{T}=10 \mathrm{~K}, \mathrm{MW}$ pulse $(\pi / 2)=20 \mathrm{~ns}, \tau=200 \mathrm{~ns}$, RF pulse $=40 \mu \mathrm{s}$, repetition time $=150 \mathrm{~ms}, 2660$ scans, $55 \mathrm{~h}$ acquisition time. Experimental Conditions (for green spectrum): $\mathrm{T}=10 \mathrm{~K}, \mathrm{MW}$ pulse $(\pi / 2)=20 \mathrm{~ns}, \tau=360 \mathrm{~ns}$, RF pulse $=40 \mu \mathrm{s}$, repetition time $=150 \mathrm{~ms}, 1700$ scans, $35 \mathrm{~h}$ acquisition time.

\section{ENDOR spectroscopy at $34 \mathrm{GHz}$ of $\alpha-\mathrm{NH}_{2} \mathrm{Y}_{730^{\circ}}$ and $\alpha-\mathrm{NH}_{2} \mathrm{Y}_{731}{ }^{\circ}$ in deuterated buffer}

Before performing the orientation selective measurements on $\mathrm{ND}_{2} \mathrm{Y}_{730^{\circ}}$ we started Q-band ENDOR experiments on $\mathrm{ND}_{2} \mathrm{Y}_{730^{\circ}}$ and $\mathrm{ND}_{2} \mathrm{Y}_{731^{\circ}}$. The EPR spectral line width changes from $75 \mathrm{G}$ at $\mathrm{W}$-band to $50 \mathrm{G}$ at Q-band frequency leading to a less pronounced g-anisotropy at Q-band. Our intention was to excite more orientations at once at this frequency and to observe changes in the deuteron hyperfine lines. Specifically, we were curious to know if the spectral line width changes, which was due to the ENDOR experiments at W-band frequency $\pm 4 \mathrm{MHz}$. In the $\mathrm{W}$-band experiment only g-tensor orientations at $\mathrm{g}_{\mathrm{y}}$ are excited, whereas the experiments at Q-band frequency allow excitation of orientations at $g_{y}, g_{x}$ and partially at $g_{z}$ (Figure 4-6). 

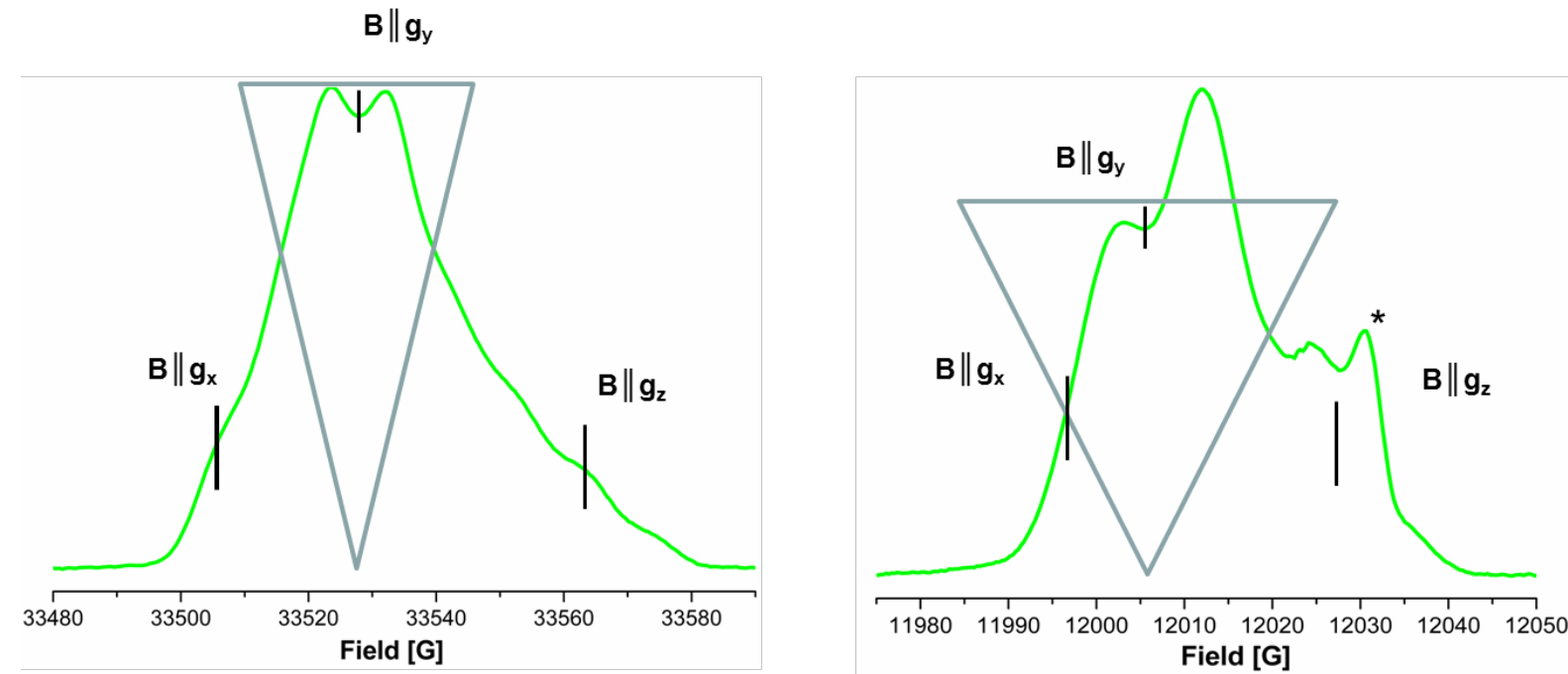

Figure 4-9. Demonstrative example of the excitation bandwidth of a $\pi / 2$ pulse on $g_{y}$ of the absorptive line of the $\mathrm{ND}_{2} \mathrm{Y}_{730^{\circ}}$ spectrum at $\mathrm{W}$-band (left) and Q-band frequency (right).The bandwidth corresponds to a $\pi / 2$ pulse of 20 ns which was used in the Mims sequence for the ENDOR experiments on $\mathrm{ND}_{2} \mathrm{Y}_{730}{ }^{\circ}$ and $\mathrm{ND}_{2} \mathrm{Y}_{731}{ }^{\circ}$ (shown below in Figure 4-7). The signal marked with an asterisk is the e-centre of a Q-band quartz tube.

Experimental conditions (for field sweep at $94 \mathrm{GHz}$ (left spectrum)): $\mathrm{T}=70 \mathrm{~K}, \mathrm{MW}(\pi / 2)=32 \mathrm{~ns}$, $\tau=260 \mathrm{~ns}, \mathrm{MW}(\pi)=64 \mathrm{~ns}$, repetition time $=3 \mathrm{~ms}$, shots $/$ point $=50$, scans $=700$. Experimental conditions (for field sweep at $34 \mathrm{GHz}$ (right spectrum)): $\mathrm{T}=70 \mathrm{~K}, \mathrm{MW}$ pulse $(\pi / 2)=32 \mathrm{~ns}, \tau=300 \mathrm{~ns}$, $\mathrm{MW}(\pi)=64 \mathrm{~ns}$, repetition time $=5 \mathrm{~ms}$, shots $/$ point $=50$, scans $=635$.

Although the Q-band approach allowed the excitation of more orientations specifically in the $g_{x}$ region we could not observe a significant effect in the ENDOR spectra of $N D_{2} Y_{730^{\circ}}$ and $N D_{2} Y_{731}{ }^{\bullet}$ (Figure 4-7). The spectral line width was slightly broader in case of $\mathrm{ND}_{2} \mathrm{Y}_{731}{ }^{\bullet}( \pm 4.1 \mathrm{MHz})$ and we could observe some change in the hyperfine tensor shape of the external hydrogen bonds (the strong signals are much broader in case of $N_{2} \mathrm{Y}_{731^{\circ}}{ }^{\circ}$. These results motivated us however to start an orientation selective study at W-band also because we were not able to excite all the orientations at once at Q-band frequency. 


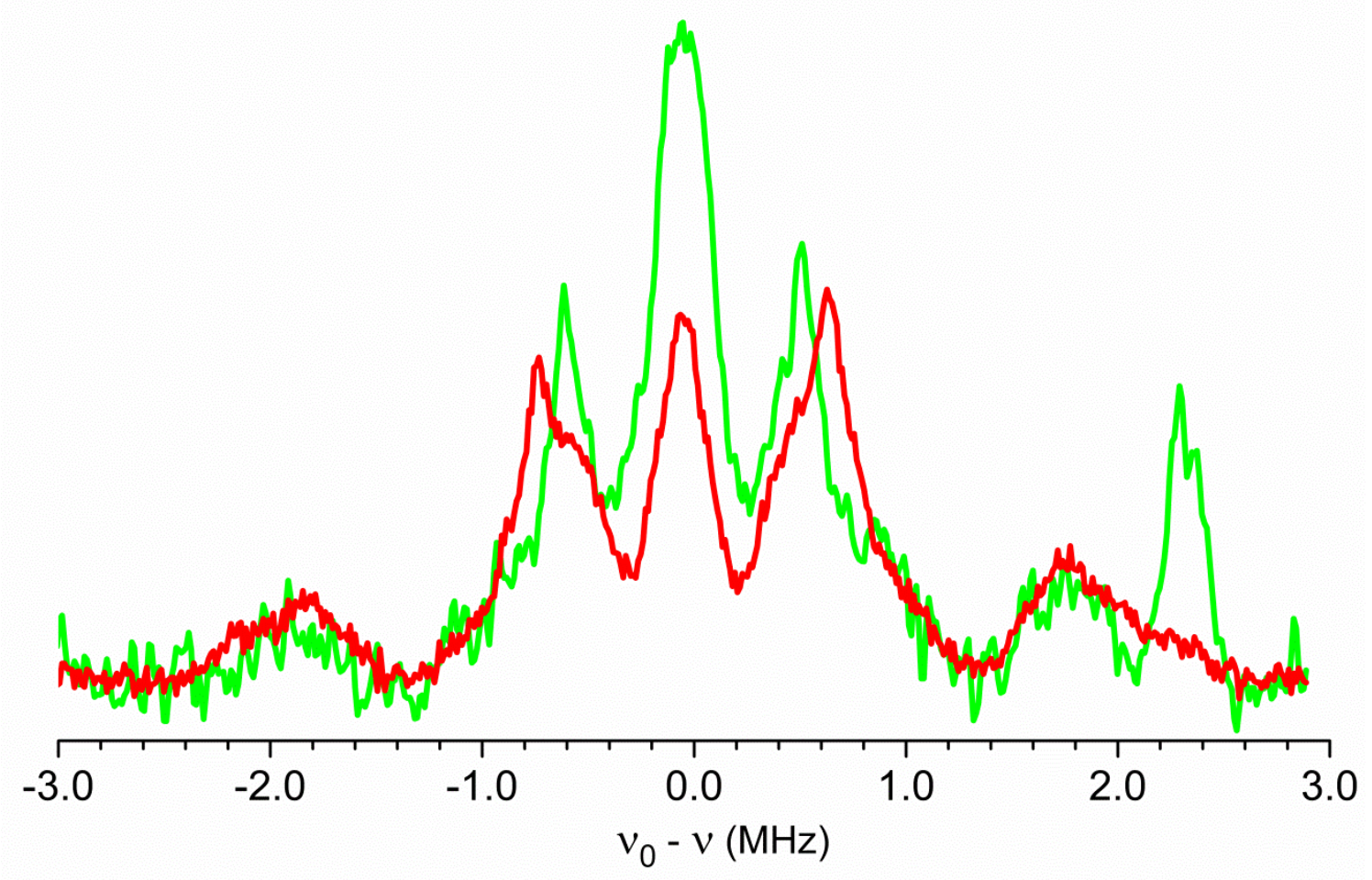

Figure 4-10. Comparison of $\mathrm{ND}_{2} \mathrm{Y}_{730}{ }^{\circ}$ and $\mathrm{ND}_{2} \mathrm{Y}_{731}{ }^{\circ}$ ENDOR spectra measured at Q-band frequency, measured with the same $\tau$-value (360 ns) as in the $\mathrm{W}$-band experiment of $\mathrm{ND}_{2} \mathrm{Y}_{730^{\circ}}$ (see Figure 4-4). The signal at $+2.4 \mathrm{MHz}$ in the $\mathrm{ND}_{2} \mathrm{Y}_{730^{\circ}}$ ENDOR experiment is due to one of several proton harmonics visible in Q-band ENDOR experiments. Experimental conditions (for ENDOR on $\mathrm{ND}_{2} \mathrm{Y}_{730^{\circ}}$ (green spectrum)): $\mathrm{T}=7.5 \mathrm{~K}, \mathrm{MW}$ pulse $(\pi / 2)=20 \mathrm{~ns}, \tau=360 \mathrm{~ns}$, RF pulse $=40 \mu \mathrm{s}$, repetition time $=150 \mathrm{~ms}$, 1700 scans, $35 \mathrm{~h}$ acquisition time. Experimental conditions (for ENDOR on $N D_{2} Y_{731}{ }^{\circ}$ (red spectrum)): $\mathrm{T}=7.5 \mathrm{~K}, \mathrm{MW}$ pulse $(\pi / 2)=20 \mathrm{~ns}, \tau=360 \mathrm{~ns}, \mathrm{RF}$ pulse $=40 \mu \mathrm{s}$, repetition time $=150 \mathrm{~ms}, 2640$ scans, $55 \mathrm{~h}$ acquisition time. Q-band ENDOR spectra were also recorded for both $\mathrm{NH}_{2} \mathrm{Y}^{\bullet}$ species with a $\tau=100 \mathrm{~ns}$ and are shown in the Appendix (Figure A-9).

\section{Inspection of the $\alpha 2-\mathrm{NH}_{2} \mathrm{Y}_{730}$ crystal structure}

The recently published crystal structure of the $\mathrm{NH}_{2} \mathrm{Y}_{730}-\alpha 2$ mutant set the boundaries for our expectations of observable deuterons in a Mims ENDOR experiment. The structure is very similar to the wt structure and suggests that in the trapped active complex several exchangeable protons can be found in a distance range on the order of $\leq 5 \AA$. After $D_{2} \mathrm{O}$ buffer exchange deuterons from functional groups of $Y_{731}, C_{439}$ and $Y_{413}$ can potentially contribute to the Mims ENDOR spectrum. From EPR experiments in $\mathrm{D}_{2} \mathrm{O}$ and $\mathrm{H}_{2} \mathrm{O}$ buffer we know already that deuterons which are part of the $\mathrm{ND}_{2}$-group contribute with large hyperfine couplings to the ENDOR spectrum. Additionally we see two water molecules which 
reside in a distance of $\leq 5 \AA$ from which one is conserved in the wt structure (Figure 4-8). In order to figure out which of the several deuterons we see during the active state of the $\alpha 2$ mutant we performed orientation selective ENDOR experiments from where we can extract the directions of $\mathrm{H}$-bonds (here $\mathrm{D}$-bonds) together with the distances. Moreover we started systematically to perform the orientation selective experiments on different spectral segments of the $\mathrm{ND}_{2} \mathrm{Y}_{730^{\circ}}$ ENDOR spectrum (see Figure 4-2).

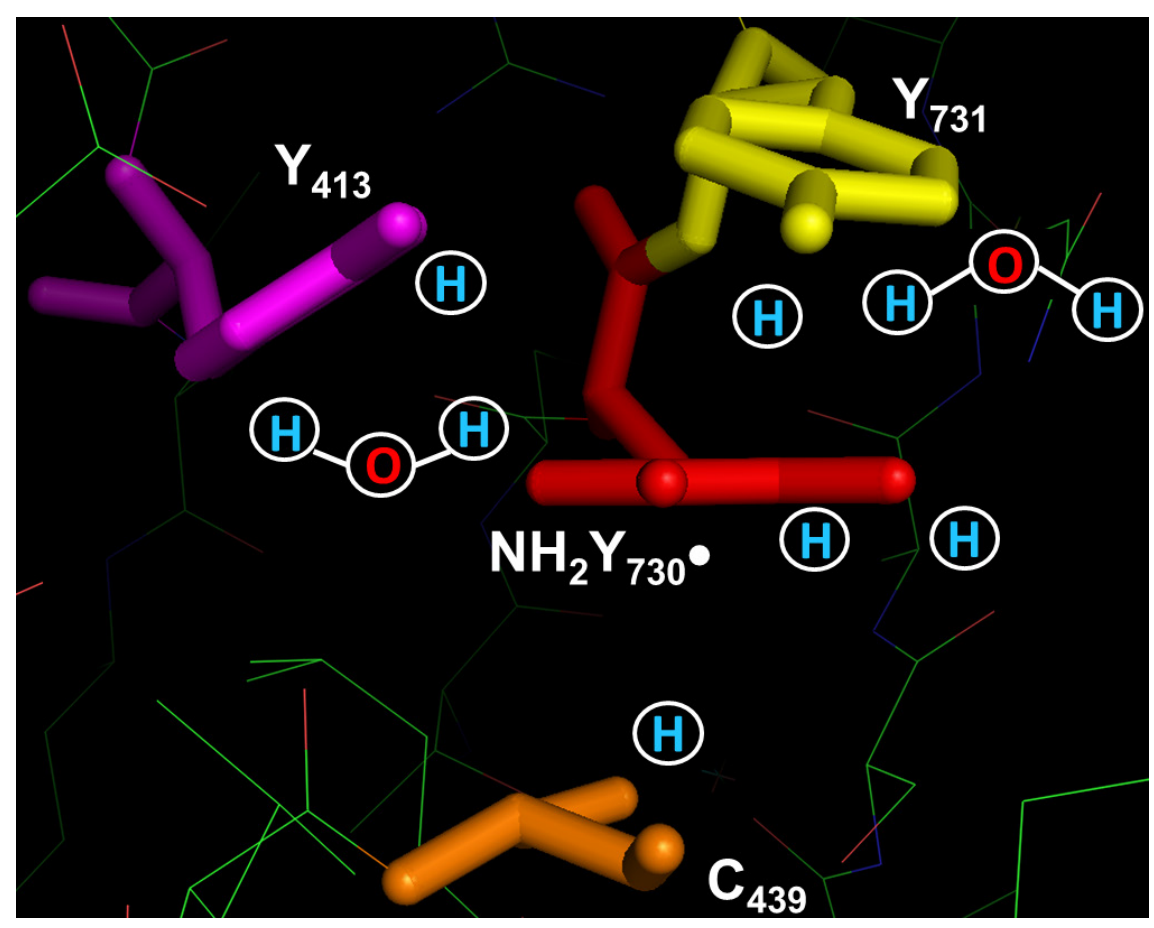

Figure 4-11. Crystal structure of $\mathrm{NH}_{2} \mathrm{Y}_{730}-\alpha 2$ together with hydrogens which could be potentially observed during activity in a high-field ENDOR experiment. The PCET pathway residues $\mathrm{Y}_{731}, \mathrm{NH}_{2} \mathrm{Y}_{730}$ and $\mathrm{C}_{439}$ are colored in yellow, red and orange respectively. The location of the substrate is below $\mathrm{C}_{439}$ (not visible). $\mathrm{Y}_{413}$ is one candidate which could potentially circumvent the proposed PCET as it is placed in 4-5 $\AA$ distance to $\mathrm{Y}_{731}$ and $\mathrm{NH}_{2} \mathrm{Y}_{730}$. The exchangeable protons which are located in a distance range of $\leq 5 \AA$ are indicated.

\section{Orientation selective high-field ENDOR on $\alpha-\mathrm{ND}_{2} \mathrm{Y}_{730^{\circ}}$ - coupling to a distant deuteron}

The weak coupling region $\left( \pm 0.30 \mathrm{MHz}\right.$ ) of the $\mathrm{ND}_{2} \mathrm{Y}_{730^{\circ}}$ ENDOR spectrum consists of a powder pattern which is typical for a single deuteron nucleus. In order to enhance the resolution in this region, the spectrum was recorded with $\Delta v=0.34 \mathrm{MHz}$ at five different field positions of the EPR line (Figure 4-9). Orientational selectivity becomes visible when 
starting at field position $\mathrm{g}_{\mathrm{x}}$ and continuing in $12 \mathrm{G}$ steps until reaching field position $\mathrm{g}_{\mathrm{z}}$. At $\mathrm{g}_{\mathrm{x}}$ the ENDOR spectrum is slightly sharper, whereas at $g_{y}$ it is broader around the centre of the Larmor frequency and finally becomes sharper again at $g_{z}$. A simulation was performed without the input of parameters from DFT calculations (model free) using hereby only one hyperfine tensor $A$, the corresponding Euler angles which indicate the direction of the major component of the A-tensor $\left(A_{z}\right)$ which lies along the $O^{\circ}-D$ distance vector and the quadrupole tensor which is collinear to $A$.

Looking at the ENDOR spectrum at $g_{x}$ one could observe for one hyperfine component a coupling of $\sim 0.4 \mathrm{MHz}$ and a quadrupole component with a coupling of $\sim 0.1 \mathrm{MHz}$. Assuming a pure dipolar tensor one could then calculate the other components of the hyperfine and quadrupole tensor and use them as input parameters for the simulation of all five ENDOR spectra.

In order to simulate all hyperfine spectra at once one had to choose also the right Euler angles. This has been performed in $5-10^{\circ}$ steps for all three angles $\alpha, \beta$, and $\gamma$. Slight changes in the $\beta$-angle showed hereby strong effects on the simulation of the orientation selective ENDOR spectra. In several iterative steps a combination of Euler angles was found which satisfied the simulations of all five ENDOR spectra. $\beta$ reflects hereby the orientation of the D-bond with respect to the $\mathrm{g}_{2}$-axis of the $\mathrm{NH}_{2} \mathrm{Y}_{730^{\circ}}$. The simulation of the orientation selective ENDOR spectra is displayed below and the analysis is consistent with a weakly coupled deuteron at a distance of $2.6 \AA \AA$. The Euler angles indicate a distance vector between the oxygen of $\mathrm{NH}_{2} \mathrm{Y}_{730^{\circ}}$ and the deuteron which is almost in plane with the aromatic ring and points to the opposite side of the $\mathrm{NH}_{2}$-group. Looking at the $\mathrm{NH}_{2} \mathrm{Y}_{730}-\alpha 2$ crystal structure one can observe one conserved water molecule between $\mathrm{NH}_{2} \mathrm{Y}_{730}$ and $\mathrm{Y}_{413}$ and one deuteron of this water molecule in an orientation which matches the obtained hyperfine and quadrupole parameters together with the Euler angles. The parameters for the simulation of the deuterium ENDOR spectrum of a $\mathrm{D}_{2} \mathrm{O}$ molecule are summarized in Table 4-1 first row. 


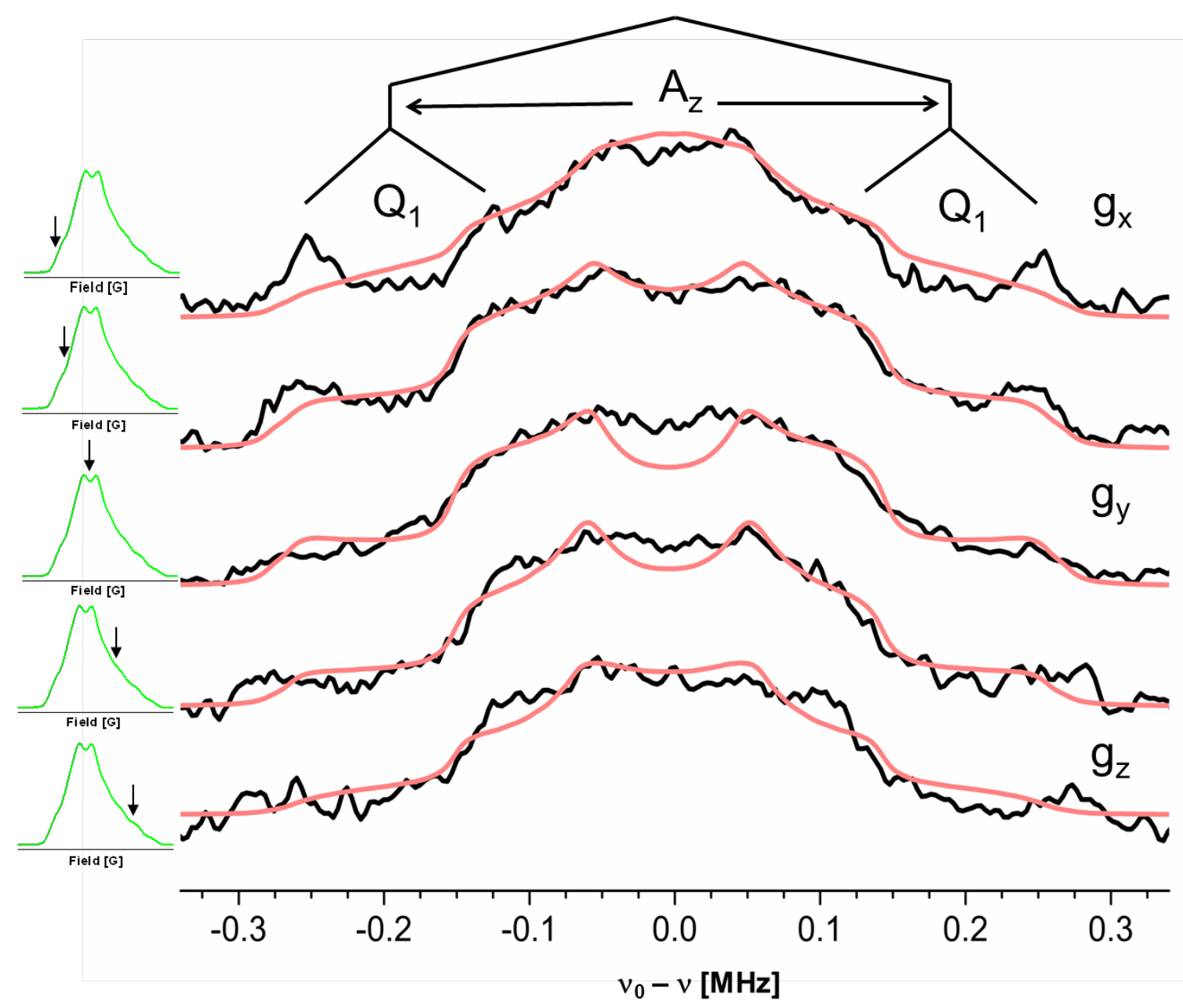

Figure 4-12. Orientation selective ${ }^{2} \mathrm{H}$ Mims ENDOR spectra of $\mathrm{NH}_{2} \mathrm{Y}_{730}{ }^{\circ}$ in $\mathrm{D}_{2} \mathrm{O}$ assay buffer of the weakly coupled region which is indicated in Figure 4-1. ENDOR spectra (black spectrum) are recorded across the EPR line in $12 \mathrm{G}$ steps. On the left side the field positions at which the ENDOR experiment was recorded are indicated. The simulations (red) were performed with the parameters from Table 4-1 for a deuteron of a $\mathrm{D}_{2} \mathrm{O}$ molecule. The largest component of the hyperfine and quadrupole coupling tensor is indicated on the first ENDOR trace. Conditions for all ENDOR spectra: $\mathrm{T}=10 \mathrm{~K}, \mathrm{MW}$ pulse $(\pi / 2)=40 \mathrm{~ns}, \tau=360 \mathrm{~ns}, \mathrm{RF}$ pulse $=40 \mu \mathrm{s}$, repetition time $=150 \mathrm{~ms}, 3000$ scans $/$ spectrum, $60 \mathrm{~h} / \mathrm{spectrum}$. 
High-field Mims ENDOR spectroscopy on $\operatorname{Tyr}_{122}{ }^{\bullet}$ in the resting state of the $\beta$-subunit compared with ENDOR spectroscopy on $\operatorname{Tyr}_{122} / \alpha-\mathrm{ND}_{2} \mathrm{Y}_{730^{\circ}}$ in the active complex

As all ENDOR spectra were recorded at $10 \mathrm{~K}$ a high probability is given that the ${ }^{2} \mathrm{H}$ resonance in the low coupling region stems from a $D_{2} \mathrm{O}$ molecule near the $\mathrm{Y}_{122}{ }^{\bullet}$ in the $\beta$-subunit. Therefore a ${ }^{2} \mathrm{H}$ Mims ENDOR spectrum has been also recorded of the $\beta$-subunit in the resting state under the same conditions like for the $\mathrm{Y}_{122}{ }^{\circ} / \mathrm{ND}_{2} \mathrm{Y}_{730^{\circ}}$ mixture in the active complex. The measurements were performed under the same experimental conditions and a comparison of the two spectra as well as a concomitant subtraction of $\mathrm{Y}_{122}$ from the $\mathrm{Y}_{122} / \mathrm{ND}_{2} \mathrm{Y}_{730^{\circ}}$ mixture, demonstrates clearly that the ${ }^{2} \mathrm{H}$ resonance of a $\mathrm{D}_{2} \mathrm{O}$ molecule is related to the $\mathrm{ND}_{2} \mathrm{Y}_{730^{\circ}}$ (see Figure 4-10). The resulting splitting pattern is a doublet of a doublet originating from one hyperfine and one quadrupole interaction.

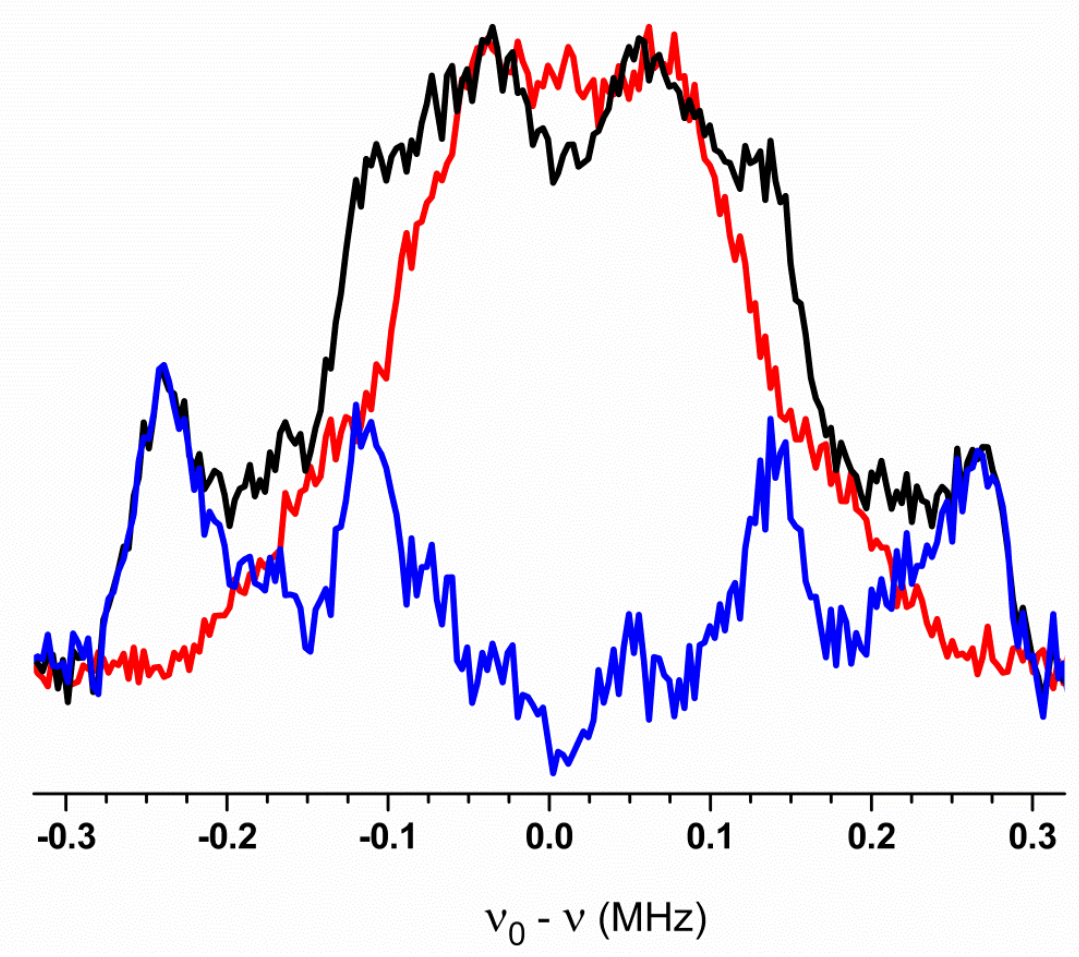

Figure 4-13. ${ }^{2} \mathrm{H}$ Mims ENDOR spectroscopy of $\alpha-\mathrm{NH}_{2} \mathrm{Y}_{730} / \beta-\mathrm{Y}_{122}{ }^{\circ}$ (black spectrum) in the active complex and of $Y_{122}{ }^{\circ}$ in the resting state of the $\beta$-subunit (red spectrum) in $D_{2} \mathrm{O}$ buffer. Both spectra were recorded with the same measurement conditions at $B \| g_{y}$. of the low coupling region Conditions for both ENDOR spectra: $\mathrm{T}=10 \mathrm{~K}, \mathrm{MW}$ pulse $(\pi / 2)=16 \mathrm{~ns}, \tau=360 \mathrm{~ns}$, RF pulse $=40 \mu \mathrm{s}$, repetition time $=150 \mathrm{~ms}, 3000$ scans/spectrum, $60 \mathrm{~h} /$ spectrum. The subtraction $\left(\mathrm{Y}_{122}{ }^{\circ} / \mathrm{ND}_{2} \mathrm{Y}_{730^{\circ}}\right.$ $\mathrm{Y}_{122}{ }^{\circ}$ ) in blue shows the quartet pattern which is also visible in Figure 4-9. 


\section{Orientation selective high-field ENDOR on $\alpha-N_{2} Y_{730^{\circ}}$ - coupling to external hydrogen bonds}

In a second series of experiments we examined the spectral region within $\pm 1.5 \mathrm{MHz}$, a region where we expect hydrogen bonds to occur. We recorded this time three ENDOR spectra across the EPR line in $18 \mathrm{G}$ steps (Figure 4-11), starting again at $g_{x}$ and observed immediately a pronounced orientation selection when going from $g_{y}$ to $g_{z}$. The strong intensity of the peaks at $\pm 0.6 \mathrm{MHz}$ at $g_{x}$ and $g_{y}$ suggested that they could constitute the perpendicular component of an axially symmetric hyperfine tensor $\left(A_{\perp}\right)$ and could arise from more than one deuteron. At field position $g_{z}$ the strong signal of the peaks at $\pm 0.6 \mathrm{MHz}$ collapsed and at $\pm 1.2 \mathrm{MHz}$ additional signals appeared given rise for the parallel component of the hyperfine tensor $\left(A_{\|}\right)$. Nevertheless, a better resolution of peaks attributable to different deuterium nuclei was not obtainable, likely due to the overlap of all signals. With this information in hand we started our simulation with two pure dipolar tensors but found also that several other combinations of hyperfine and quadrupole tensor components as well as Euler angles could satisfy the simulation with similar quality. We needed therefore the input of parameters for external hydrogen bonds which were calculated through the DFT methodology. The obtained parameters are based on a quantum chemical calculation of an optimized structure of the $\mathrm{NH}_{2} \mathrm{Y}_{730} 0^{\circ}$ together with several surrounding amino acids including the PCET pathway residues and the water molecules which are visible in the $\mathrm{NH}_{2} \mathrm{Y}_{730^{-}}-\alpha 2$ crystal structure.

The different DFT cluster models and specifically their effect on the $\mathrm{g}_{x}$-value of $\mathrm{NH}_{2} \mathrm{Y}_{730^{\circ}}$, which is an indicator for $\mathrm{H}$-bond formations is explained in a separate section (see below). However as documented in Table 4-1 we leaned on the DFT results from the calculation of Model 1. Atypical hyperfine tensors for both deuterons ( $\mathrm{Tyr}_{731}-\mathrm{OD}$ and $\mathrm{Cys}_{439}-\mathrm{SD}$ ) were reported which consisted of a significant isotropic part $\left(A_{\text {iso }}\right)$, due to strong contact interactions of both deuterons with the aromatic $\pi$-system. The shape of the hyperfine tensors was retained but some hyperfine tensor components had to be increased to about $0.5 \mathrm{MHz}$ in order to simulate the spectra. We used hereby small model systems (see below) where the hyperfine tensor is calculated with respect to the bond distance and the out-ofplane angle (angle between the bond and the aromatic ring). The quadrupole tensors were 
obtained with the same approach and the Euler angles through inspection of the X-ray structure.

Specifically the $\beta$ angle could be estimated from the $\mathrm{X}$-ray structure and was then compared with the obtained $\beta$ angle from the DFT cluster calculation. Other angles were obtained through systematic change in $10^{\circ}$ steps and were also compared with the results from the DFT cluster calculation. Finally the simulation was also deconvoluted in order to demonstrate the separate contributions to the overall simulation of the two deuterons $\left(\mathrm{Y}_{731}-\mathrm{OD}\right.$ and $\left.\mathrm{C}_{439}-\mathrm{OD}\right)$.

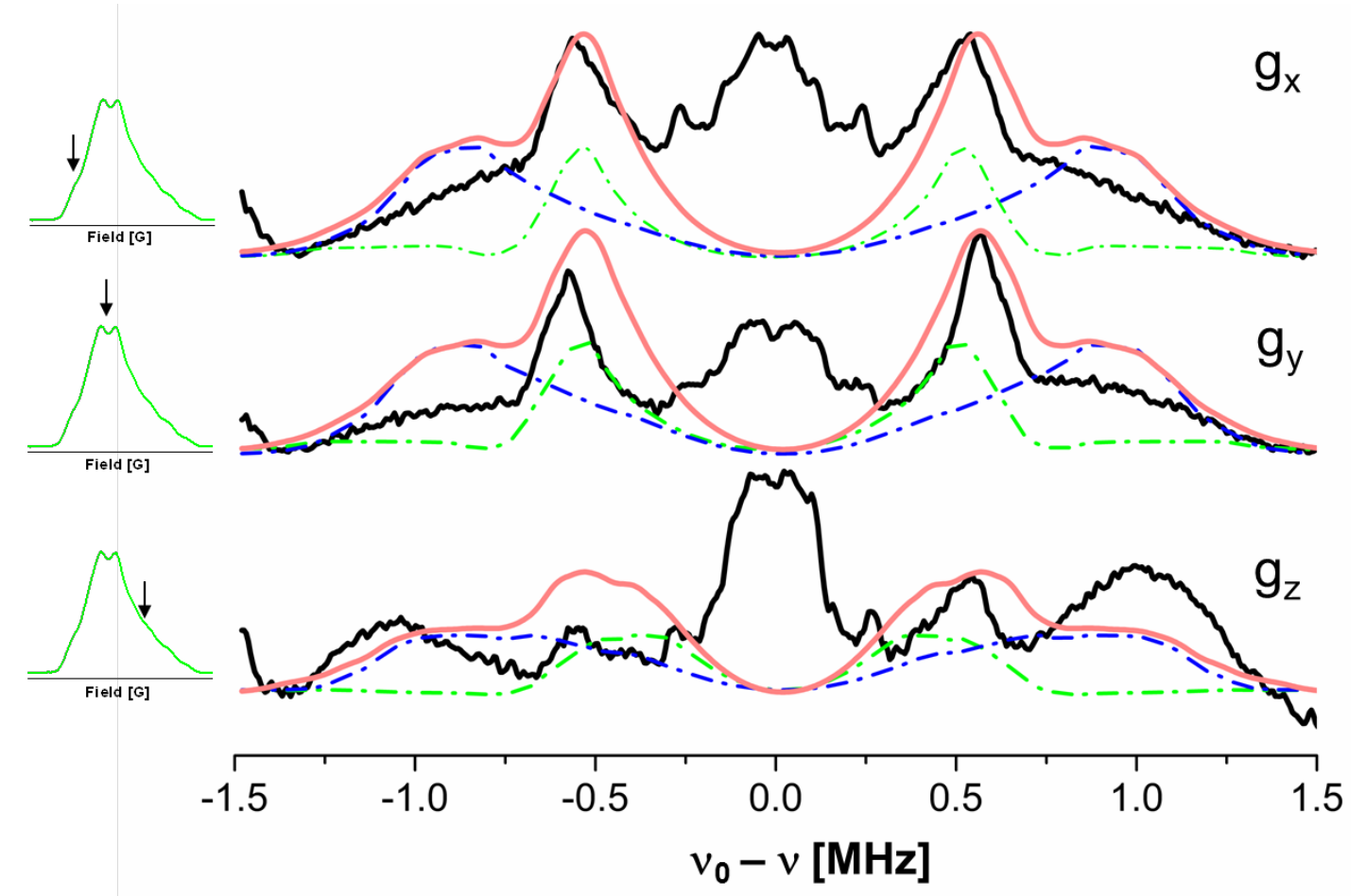

Figure 4-14. Orientation selective ${ }^{2} \mathrm{H}$ Mims ENDOR spectra of $\mathrm{NH}_{2} \mathrm{Y}_{730^{\circ}}$ in $\mathrm{D}_{2} \mathrm{O}$ assay buffer in the region $\pm 1.5 \mathrm{MHz}$ where external hydrogen bonds are expected. Spectra were recorded across the EPR line in $18 \mathrm{G}$ steps (black spectra). On the left side the field positions at which the ENDOR experiment was recorded are indicated. The simulation (red) has been deconvoluted in two separate simulations (blue and green) with the parameters for $\mathrm{Y}_{731}$-OD (blue spectrum) and $\mathrm{C}_{439}$-OD (green spectrum) as listed in Table 4-1. In the two separate simulations hyperfine and quadrupole parameters as well as Euler angles for the exchangeable hydrogens of the $\mathrm{NH}_{2}$-group were not included (see Table 4-1). Conditions for all ENDOR spectra: $\mathrm{T}=10 \mathrm{~K}$, MW pulse $(\pi / 2)=20 \mathrm{~ns}, \tau=320$ $\mathrm{ns}, \mathrm{RF}$ pulse $=40 \mu \mathrm{s}$, repetition time $=150 \mathrm{~ms}, 2500$ scans $/$ spectrum, $50 \mathrm{~h} / \mathrm{spectrum}$. 


\section{Orientation selective high-field ENDOR on $\alpha-N_{2} Y_{730^{\circ}}$ - coupling to deuterons of the}

\section{$\mathrm{ND}_{2}-$ group}

In the last series of experiments we recorded orientation selective ENDOR spectra of the whole spectral region where also the resonances of the amino deuterons appear. Orientational selectivity appears when going from $g_{x}$ to $g_{z}$ over $g_{y}$ (Figure 4-12). The resonances between \pm 1.0 and $\pm 2.0 \mathrm{MHz}$ changed in intensity with respect to the strong signals at $\pm 0.6 \mathrm{MHz}$ and additionally the spectral line width of the spectrum changed from \pm 2.2 $\mathrm{MHz}$ to $\pm 1.8 \mathrm{MHz}$. For the simulation we took the previously determined parameters from Table 4-1 for the deuterons of $\mathrm{Tyr}_{731}-\mathrm{OD}$ and $\mathrm{Cys}_{439}-\mathrm{SD}$ together with the known parameters for the deuterons of the $\mathrm{ND}_{2}$-group. $A_{x}$ of $N_{2}(D 2)$ had to be optimized from 2 to $3 \mathrm{MHz}$ in deuteron frequency and other values were varied a little. However the tensor shape for both $\mathrm{ND}_{2}$ deuterons was retained with respect to Table 3-1 in Chapter 3.

A satisfactory simulation was obtained for all three ENDOR traces where all significant resonances are represented in the simulation as well as the form of several overlayed hyperfine tensors. Moreover exactly the same Euler angles could be used which were used in the high-field EPR simulation of $\mathrm{NH}_{2} \mathrm{Y}_{730^{\circ}}$ in $\mathrm{H}_{2} \mathrm{O}$ buffer. The quadrupole values which are slightly smaller with respect to the DFT calculations have been also implemented.

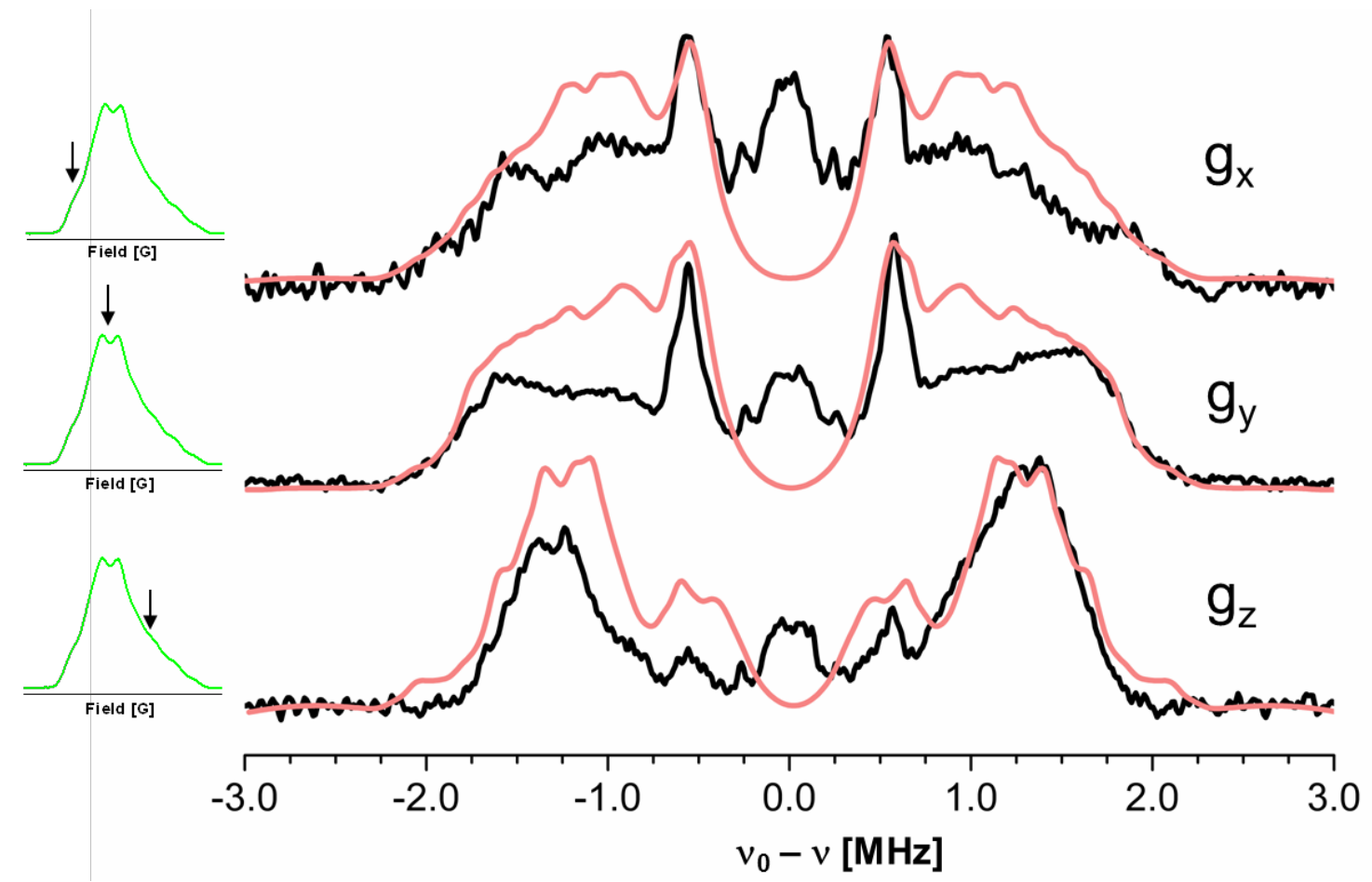

Figure 4-15. Orientation selective ${ }^{2} \mathrm{H}$ Mims ENDOR spectra of $\mathrm{NH}_{2} \mathrm{Y}_{730^{\circ}}$ in $\mathrm{D}_{2} \mathrm{O}$ assay buffer of the whole spectral region. Spectra were recorded across the EPR line in $18 \mathrm{G}$ steps (black spectra). On 135 | P a g e 
the left side the field positions at which the ENDOR experiment was recorded are indicated. The simulations (red) include the parameters for the $\mathrm{ND}_{2}$-group and for $\mathrm{Y}_{731}-\mathrm{OD}$ and $\mathrm{C}_{439}-\mathrm{OD}$.

Conditions for all ENDOR spectra: $\mathrm{T}=10 \mathrm{~K}$, MW pulse $(\pi / 2)=20 \mathrm{~ns}, \tau=200 \mathrm{~ns}$, RF pulse $=40 \mu \mathrm{s}$, repetition time $=150 \mathrm{~ms}, 2500$ scans/spectrum, $50 \mathrm{~h} / \mathrm{spectrum}$.

The parameters which were used in the simulations of the three orientation selective ENDOR experiments together with the calculated DFT parameters according to Model 1 are summarized in Table 4-1. The obtained O-D and S-D distances from $\mathrm{Y}_{731}$ and $\mathrm{C}_{439}$ respectively are in very good agreement within $0.1 \AA$ difference. For the deuteron of the $\mathrm{D}_{2} \mathrm{O}$ molecule which is residing near to the nodal plane of the aromatic $\pi$-system (no contact interaction) we were able to calculate the $\mathrm{ND}_{2} \mathrm{Y}_{730}-\mathrm{O}^{\circ}$-D distance using the point-dipole formula for pure dipolar interaction between radical and deuteron (Equation 4-1). The discrepancy to the distance obtained through DFT calculations is quite significant $(0.4 \AA)$ and is an important point in the upcoming discussion.

$$
r=\sqrt[3]{\frac{\mu_{0} \cdot g_{e} \cdot \beta_{e} \cdot g_{n} \cdot \beta_{n} \cdot \rho_{O}}{4 \pi \cdot A_{\perp}}} \quad r=\sqrt[3]{\frac{3.558}{A_{\perp}}}
$$

Equation 4-1. Point-dipole model formula considering the dipole interaction between one nucleus and the electron spin density of the oxygen of $\mathrm{NH}_{2} \mathrm{Y}_{730^{\circ}}$. The bond distances around the spin trap are calculated with the assumption of an oxygen spin density $\left(\rho_{0}\right)$ of around $0.3(10) . \mu_{0}$ is hereby the permeability constant of vacuum. $g$ and $\beta$ are the nuclear and electronic g-factors and magnetons, respectively. $A_{\perp}$ (or $A_{x}$ ) is the experimental input parameter (perpendicular hyperfine tensor component) for the calculation of a point dipole distance $r$. Specific point dipole expression for deuterium (right) where all the physical constants together with $\rho_{0}$ are summarized in the enumerator. 


\begin{tabular}{|c|c|c|c|c|c|c|c|c|c|c|}
\hline & $A_{x}$ & $A_{y}$ & $A_{z}$ & $\alpha$ & $\beta$ & $\gamma$ & $Q_{1}$ & $Q_{2}$ & $Q_{3}$ & $r_{O-D}$ \\
\hline $\mathbf{D}_{2} \mathbf{O}$ & & & & & & & & & & \\
\hline Sim & -0.21 & -0.21 & 0.42 & 55 & 100 & -65 & 0.10 & -0.04 & -0.05 & $2.6^{(\mathrm{a})}$ \\
\hline $\begin{array}{c}\text { DFT } \\
\text { Model 1 }\end{array}$ & -0.31 & -0.28 & 0.64 & -6 & 81 & -50 & 0.14 & -0.06 & -0.07 & 2.2 \\
\hline $\begin{array}{c}\mathbf{T y r}_{73^{-}} \\
\text {OD }\end{array}$ & & & & & & & & & & \\
\hline Sim & -2.38 & -1.82 & 1.18 & -10 & 45 & 75 & -0.04 & -0.06 & 0.10 & $1.7^{(b)}$ \\
\hline $\begin{array}{c}\text { DFT } \\
\text { Model 1 }\end{array}$ & -1.70 & -1.15 & 0.73 & -14 & 47 & 78 & -0.04 & -0.06 & 0.11 & 1.8 \\
\hline Cys $_{439}$-SD & & & & & & & & & & \\
\hline Sim & -1.18 & -1.12 & 0.78 & -10 & 160 & 85 & 0.06 & -0.03 & -0.03 & $2.0^{(6)}$ \\
\hline $\begin{array}{c}\text { DFT } \\
\text { Model 1 }\end{array}$ & -1.04 & -0.78 & 0.64 & -18 & 142 & 44 & 0.07 & -0.04 & -0.03 & 2.0 \\
\hline $\mathbf{N D}_{\mathbf{2}}$ (D2) & & & & & & & & & & \\
\hline Sim & 3.02 & 0.67 & 4.37 & 0 & 126 & -60 & 0.08 & -0.04 & -0.04 & - \\
\hline $\mathbf{N D}_{\mathbf{2}}$ (D1) & & & & & & & & & & \\
\hline Sim & 1.04 & 1.23 & 3.39 & 0 & 50 & 0 & 0.08 & -0.04 & -0.04 & - \\
\hline
\end{tabular}

Table 4-1. Parameter set for deuterons which are a constituent of the external hydrogen-bond network and part of the 3-aminotyrosyl radical at position 730 . The hyperfine and quadrupole values are given in $\mathrm{MHz}$ and the corresponding Euler angles $(\alpha, \beta, \gamma)$ are given in degrees. The signs of the couplings from the simulation are known only relative to each other within one tensor. The conversion factor from ${ }^{1} \mathrm{H}$ to ${ }^{2} \mathrm{H}$ frequency is 6.5 with respect to Table 3-1. Distances are given in $\AA ̊$ and errors are estimated approximately to be 5-10 \%. (a) from point-dipole model; (b) from DFT calculations on the angular and distance dependence of $A$ and $Q$ values of small model systems.

DFT calculations explain the experimental g-values and confirm hydrogen-bonds to the

\section{3-aminotyrosyl radical in the protein environment}

As demonstrated in the introduction the $g_{x}$-value of a radical is very sensitive to hydrogenbond interactions. In an attempt to understand our g-values of $g_{x}=2.0052, g_{y}=2.0042$ and $g_{z}=2.0022$ which were obtained from the $180 \mathrm{GHz}$ experiment, DFT calculations have been first performed on small model systems starting from the 4-methyl phenol radical (Table 42). Further, calculations were performed on the isolated 2-amino-4-methylphenol radical (2AMPR) which is mimicking our 3-aminotyrosyl radical in the isolated state (without the protein environment) together with different 2-AMPR hydrogen-bond complexes using the structural restraints already available from big model systems. In a second series of calculations the protein environment around the $\mathrm{NH}_{2} \mathrm{Y}_{730^{\circ}}$ was taken into account (DFT cluster calculations) with six additional amino acids, including $\mathrm{Tyr}_{731}$ and $\mathrm{Cys}_{439}$. We took into consideration for the calculation all amino acid residues which had at least one atom located 
in a $5 \AA$ radius. The two water molecules WAT1 and WAT2 which are present in the $\mathrm{NH}_{2} \mathrm{Y}_{730^{-}}$ $\alpha 2$ crystal structure are also included in the cluster calculations (see for details Materials and Methods part). WAT1 and WAT2 are located left and right from the amino-group of $\mathrm{NH}_{2} \mathrm{Y}_{730}{ }^{\circ}$ and the six calculated models differ hereby in the number and position of water molecules (Figure 4-13).

With respect to the small model systems we observe a substantial $g_{x}$-shift when the aminogroup is introduced in ortho-position of the 4-methylphenol radical. The $g_{x}$-value decreases from 2.0092 to 2.0069 and even further to $2.0060,2.0055$ and 2.0050 when one or two hydrogen bonds are included. The water molecule in $2.2 \AA$ distance decreases $g_{x}$ further to 2.0046 what is much lower than experimentally determined.

\begin{tabular}{|c|c|c|c|c|}
\hline DFT model structure & $g_{x}$ & $\mathrm{~g}_{\mathrm{y}}$ & $\mathrm{g}_{\mathrm{z}}$ & radical \\
\hline & 2.0092 & 2.0047 & 2.0022 & 4-methyl-phenol radical \\
\hline & 2.0069 & 2.0046 & 2.0022 & $\begin{array}{l}\text { 2-aminodimethyl-4-methyl- } \\
\text { phenol radical }\end{array}$ \\
\hline & 2.0061 & 2.0045 & 2.0022 & $\begin{array}{l}\text { 2-amino-4-methyl-phenol } \\
\text { radical (2-AMPR) }\end{array}$ \\
\hline & 2.0060 & 2.0043 & 2.0018 & $2-A M P R+C y s_{439}$ \\
\hline & 2.0055 & 2.0044 & 2.0020 & 2-AMPR + $\mathrm{Tyr}_{731}$ \\
\hline & 2.0050 & 2.0040 & 2.0018 & $2-A M P R+T^{2} r_{731}+C_{y s} s_{439}$ \\
\hline & 2.0046 & 2.0039 & 2.0017 & $2-\mathrm{AMPR}+\mathrm{Tyr}_{731}+\mathrm{Cys}_{439}+\mathrm{H}_{2} \mathrm{O}$ \\
\hline Experiment & 2.0052 & 2.0042 & 2.0022 & \\
\hline
\end{tabular}

Table 4-2. Summarized g-values from small model systems (DFT calculations) and from experiment. The decrease of the $g_{x}$-value is indicated, starting from 4-methyl-phenol radical and ending with $\mathrm{NH}_{2} \mathrm{Y}_{730^{\circ}}$ surrounded by $\mathrm{Y}_{731}, \mathrm{C}_{439}$ and $\mathrm{H}_{2} \mathrm{O}$ in hydrogen bonding distance. A change in the $\mathrm{g}_{\mathrm{y}}$-value is 138 | P a g e 
also present but less pronounced. The small models are shown on the left and named on the right side.

The different model systems are summarized below (Figure 4-13) together with the g-values which were all in close agreement with our spectroscopic results. Model 1 with one water molecule (WAT1) is in very good agreement with the results obtained from high-field EPR and ENDOR measurements, however placing the water molecule in a closer distance than in the active enzymatic state $\left(\mathrm{O}^{\circ}-\mathrm{D}-\mathrm{OD}\right.$ distance is 2.2 instead $2.6 \AA$ ). Importantly, the best agreement between theory and experiment is given if one takes Model 6 into account where no water molecule is included, suggesting that our experimentally observed $D_{2} \mathrm{O}$ molecule is not in hydrogen bond distance range. This fits very good with the observation that the $g_{x}$-value in Model 1 (2.0049) falls below the $g_{x}$-value of 2.0052 . We see therefore the decreasing of this $g_{x}$-value represented in the shorter $0^{\circ}-\mathrm{D}_{2} \mathrm{O}$ distance of $2.2 \AA$ in the resting state of the enzyme (weak hydrogen bond). Model 2, 3 and 4 consider all two water molecules in the calculation. One is placed as in Model 1 between $\mathrm{Y}_{413}$ and $\mathrm{NH}_{2} \mathrm{Y}_{730}{ }^{\circ}$ and the second water molecule resides on the site of the amino-group as observed in the $\mathrm{NH}_{2} \mathrm{Y}_{730}-\alpha 2$ crystal structure.

All three structures with two water molecules were not considered for further analysis because they show either a strong interaction with the amino group of $\mathrm{NH}_{2} \mathrm{Y}_{730}{ }^{\circ}$ or with the carbonyl group of $A_{695}$. The first situation gives rise to nitrogen tensors and $C_{\beta}$-methylene proton tensors of $\mathrm{NH}_{2} \mathrm{Y}_{730^{\circ}}$ which are not compatible with our EPR results.

A very similar calculation to Model 1 is represented through Model 5 . The $0^{\circ}$-D-OD distance is $1.9 \AA$ long and $0.7 \AA$ shorter with respect to our experimental results.

Although both calculated dipolar tensors (Model 1 and Model 5) are not in agreement with our experimental one, we point out that Model 1 with the deuteron placed $2.2 \AA$ from the oxygen of the $\mathrm{NH}_{2} \mathrm{Y}_{730^{\circ}}$ comes nearer to our results from the ENDOR experiment.

In summary DFT calculations on small model systems and protein clusters (in total 137 atoms) have been performed in order to explain the significantly low $g_{x}$-value observed in our $180 \mathrm{GHz}$ EPR experiment considering hereby the location of the amino-group in orthoposition, an external hydrogen bonding network around the $\mathrm{NH}_{2} \mathrm{Y}_{730}{ }^{\circ}$ together with the surrounding protein environment. 
As mentioned above the DFT calculations provided us also with the important information about the shape of the two hyperfine tensors of $\mathrm{Tyr}_{731}-\mathrm{OD}$ and $\mathrm{Cys}_{439}-\mathrm{SD}$. Still the calculated hyperfine tensor for the deuteron of the water molecule exceeds far the tensor which we observe experimentally.

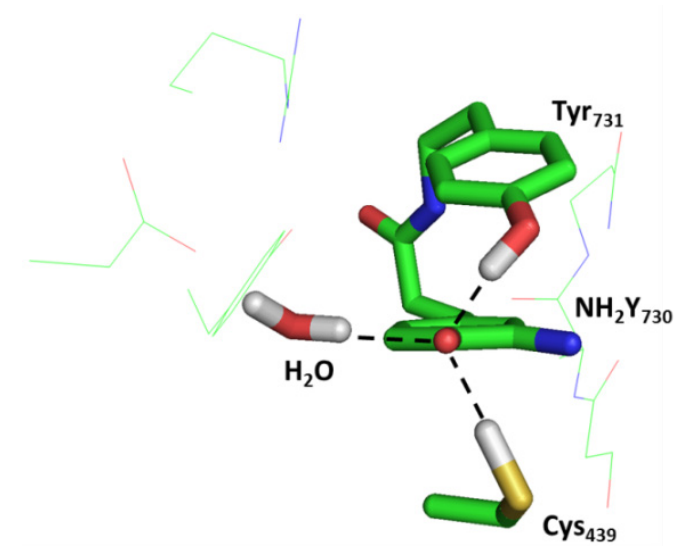

Model 1

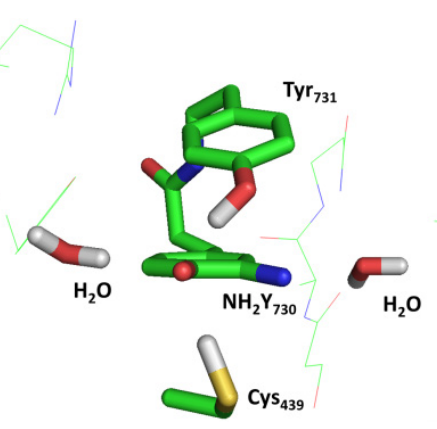

Model 2

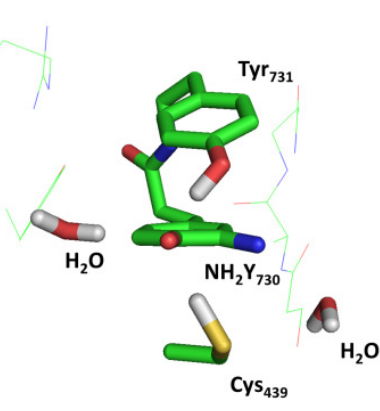

Model 3

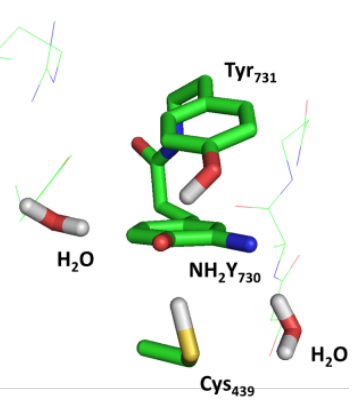

Model 4

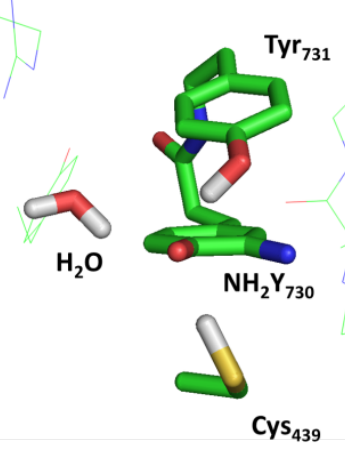

Model 5

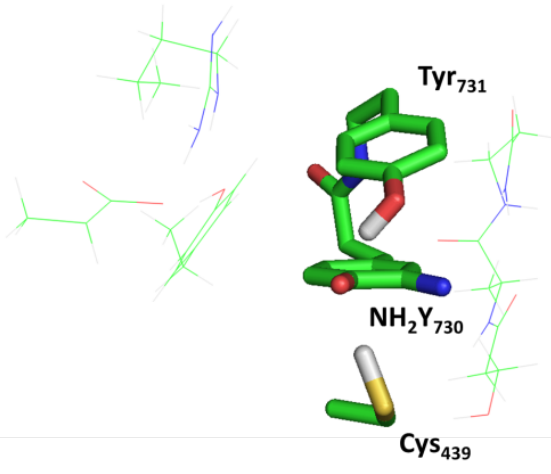

Model 6 
Figure 4-16. Summary of six calculated DFT cluster models which take the protein environment and water molecules into account around the $\mathrm{NH}_{2} \mathrm{Y}_{730^{\circ}}$. The PCET pathway residues together with the water molecules are shown in bold. Additional amino acids are shown in stick representation. All hydrogens besides the one which form a hydrogen bond network around $\mathrm{NH}_{2} \mathrm{Y}_{730}$ are omitted. Calculations result in g-values which are close to the experimental one $\left(g_{x}=2.0052, g_{y}=2.0042\right.$ and $\left.g_{z}=2.0022\right)$. The g-values for all models and the model characteristics are displayed below in Table 4-3.

In conclusion six DFT cluster models were constructed in order to find an optimized structure which is compatible with the orientation selective ENDOR spectra of $\alpha-\mathrm{NH}_{2} \mathrm{Y}_{730^{\circ}}$. All structures used the coordinates of $\mathrm{NH}_{2} \mathrm{Y}_{730}-\alpha 2$ as input but differed in the number and arrangement of the water molecules. Only one structure did not take a water molecule into account. Two structures contained only one water molecule located in hydrogen bond distance between $\mathrm{Y}_{413}$ and $\mathrm{NH}_{2} \mathrm{Y}_{730}$ and three other structures took into account a second water molecule visible in the $\mathrm{NH}_{2} \mathrm{Y}_{730}-\alpha 2$ structure but not in the wt structure. In these three structures the $\mathrm{NH}_{2} \mathrm{Y}_{730^{-}} \mathrm{O}^{\circ}$ - D-OD bond is $~ 5 \AA$ long and we are at the limit for the observation of deuterium resonances in ${ }^{2} \mathrm{H}$ Mims ENDOR experiments. Moreover in two of the structures the water molecule is attached to the $\mathrm{NH}_{2}$-group and changes the $\mathrm{C}_{\alpha}$ - $\mathrm{C}_{\beta}$-ring-plane dihedral angle in such a way that we observe isotropic couplings for $C_{\beta}$-methylene protons of up to 50 $\mathrm{MHz}$. This is $20 \mathrm{MHz}$ more than what we used as input for the multifrequency EPR analysis of $\mathrm{NH}_{2} \mathrm{Y}_{730^{\circ}}$. Additionally in one of the calculations the $\mathrm{A}_{\mathrm{z}}$ value of the ${ }^{14} \mathrm{~N}$ tensor is lower, about $10 \mathrm{MHz}$ with respect to the one which we used in the simulations.

\begin{tabular}{|c|c|c|c|c|}
\hline g-values/models & $g_{x}$ & $\mathbf{g}_{\mathrm{y}}$ & $g_{z}$ & model features \\
\hline 1 & 2.0049 & 2.0041 & 2.0018 & D- $0^{\circ}$ distance $=2.2 \AA$ \\
\hline 2 & 2.0049 & 2.0041 & 2.0018 & $\begin{array}{c}2^{\text {nd }} \text { water attached to } \\
C=O \text { group of } A_{695}\end{array}$ \\
\hline 3 & 2.0048 & 2.0038 & 2.0018 & $\begin{array}{l}2^{\text {nd }} \text { water attached } \\
\text { through the oxygen to } \\
\text { the } \mathrm{NH}_{2} \text {-group of } \\
\mathrm{NH}_{2} \mathrm{Y}_{730^{\circ}}\end{array}$ \\
\hline 4 & 2.0055 & 2.0041 & 2.0019 & $\begin{array}{c}2^{\text {nd }} \text { water attached } \\
\text { through the hydrogen } \\
\text { to the } \mathrm{NH}_{2} \text {-group of } \\
\qquad \mathrm{NH}_{2} \mathrm{Y}_{730^{\circ}}\end{array}$ \\
\hline 5 & 2.0048 & 2.0040 & 2.0018 & $\mathrm{D}-\mathrm{O}^{\circ}$ distance $=1.9 \AA$ \\
\hline 6 & 2.0053 & 2.0042 & 2.0018 & no water molecule \\
\hline
\end{tabular}


Table 4-3. Summary of g-values resulting from calculations of six DFT cluster models together with model features.

The two structures with one water molecule differed in the $\mathrm{NH}_{2} \mathrm{Y}_{730^{-}} \mathrm{O}^{\circ}$ - D-OD distance which was 1.9 and $2.2 \AA$, respectively. However the structure with the longer distance generated a dipolar tensor together with the deuteron tensors for the OD- and SD-group, which came quite close to the experimental values. This structure with the hydrogen network around $\mathrm{NH}_{2} \mathrm{Y}_{730^{\circ}}$ is shown below (Figure 4-14).

Still remarkably, the calculated DFT structure with no water molecule demonstrates through comparison with our experimental g-values that a calculation with a water molecule with a $\mathrm{NH}_{2} \mathrm{Y}_{730}-\mathrm{O}^{\circ}-\mathrm{D}-\mathrm{OD}$ distance of $2.6 \AA$ could be indicative for no hydrogen-bond formation.

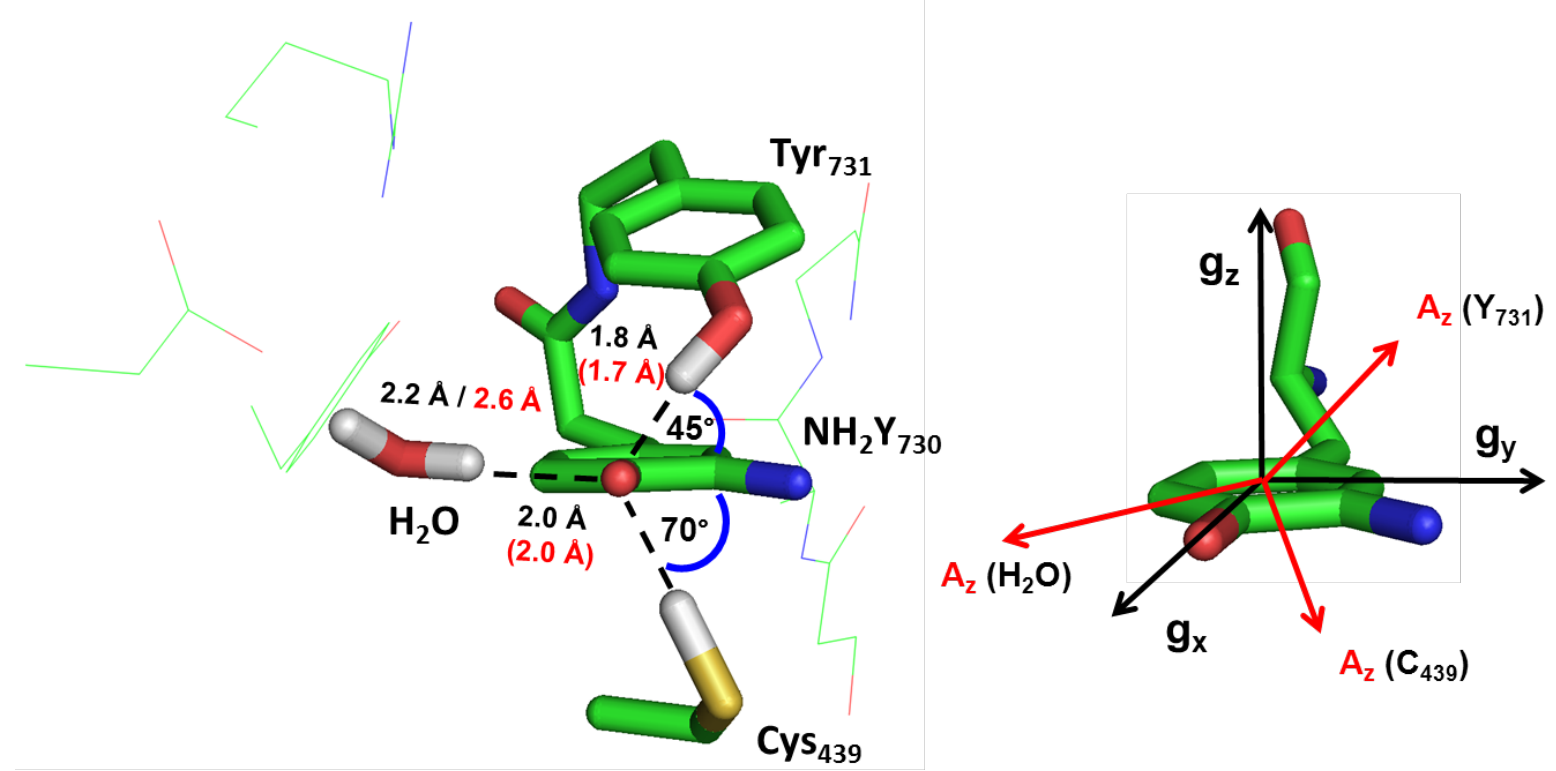

Figure 4-17. Combined EPR and DFT model for the hydrogen-bond network around $\mathrm{NH}_{2} \mathrm{Y}_{730^{\circ}}$ in the $\alpha$-subunit. (Left) DFT distances are indicated in black for the resting state of the $\alpha$-subunit and ENDOR distances are indicated in red for the active state of the E.coli RNR enzyme. The out-of-plane angles formed between the two hydrogen bonds and the aromatic ring plane are obtained from the $\beta$-angles of the simulation in Table 4-1. (Right) Position of the g-tensor within the $\mathrm{NH}_{2} \mathrm{Y}$ structure. The direction of the hyperfine tensor component $A_{z}$ which lies in bond direction is also schematically drawn with red arrows in the g-tensor frame. 


\section{Orientation selective high-field ENDOR on $\alpha-\mathrm{ND}_{2} \mathrm{Y}_{731}{ }^{\circ}$ - coupling to a distant deuteron}

Taking into consideration Figure 4-3 it is not obvious like in Figure 4-2 for $N_{2} \mathrm{Y}_{730}{ }^{\circ}$ that the low coupling region consists of a powder pattern of a far deuteron. We have recorded therefore also here highly resolved orientation selective ENDOR spectra of the weak coupling region of $\mathrm{ND}_{2} \mathrm{Y}_{731}{ }^{\circ}$ in order to probe for a deuteron distance and orientation which is for sure further apart than $2.6 \AA$ (compare edges of the orientation selective spectra of Figure 4-9 and 4-15). The measurements demonstrate clear orientation selection when going from $g_{x}$ to $g_{z}$ in $12 \mathrm{G}$ steps (see Figure 4-15) although the orientation selection is less pronounced between $g_{y}$ and $g_{z}$. Between $g_{x}$ and $g_{y}$ the ENDOR spectra show a maximum edge-to-edge distance of $\pm 0.2 \mathrm{MHz}$. The orientation selective spectra could be simulated using one hyperfine and quadrupole tensor $A$ and $Q$ and the corresponding Euler angles indicating the $\mathrm{O}^{\circ}-\mathrm{D}$ distance vector to lie in a direction nearly perpendicular to the aromatic $\pi$-plane. At this distance we assume a pure dipolar tensor as the deuteron should not interact with the $\pi$-orbitals of the $\mathrm{NH}_{2} \mathrm{Y}_{731}$ ' for distances longer than $2.4 \AA$ (see Appendix). Also here the simulation was performed without the input of parameters from DFT calculations but with the information of possible water locations in the X-ray structure of $\alpha 2$ $\mathrm{NH}_{2} \mathrm{Y}_{731}$. In one molecule of the asymmetric crystal structure unit, one oxygen of a water molecule is placed in a distance of $4.9 \AA$ from the oxygen and $3.5 \AA$ from the nitrogen of $\mathrm{NH}_{2} \mathrm{Y}_{731}$. Through a systematic change of the $\beta$ - and $\gamma$-angle and with respect to these distances we could obtain an ENDOR simulation with a deuteron which is $3.5 \AA$ away from the oxygen of $\mathrm{NH}_{2} \mathrm{Y}_{731}$. However also here an analysis through DFT calculations (small model systems), taking the crystal structure coordinates of $\mathrm{NH}_{2} \mathrm{Y}_{731}-\alpha 2$ as input would be very important in order to confirm the distances obtained from the ENDOR simulations. Additionally we have to emphasize that the tensor which we use for the far deuteron is significantly masked by the deuterium matrix peak which is strongly pronounced around the deuterium resonance line and which aggravated our analysis. The parameters for the simulation of the deuteron of a $\mathrm{D}_{2} \mathrm{O}$ molecule are summarized in Table 4-4 first row. 


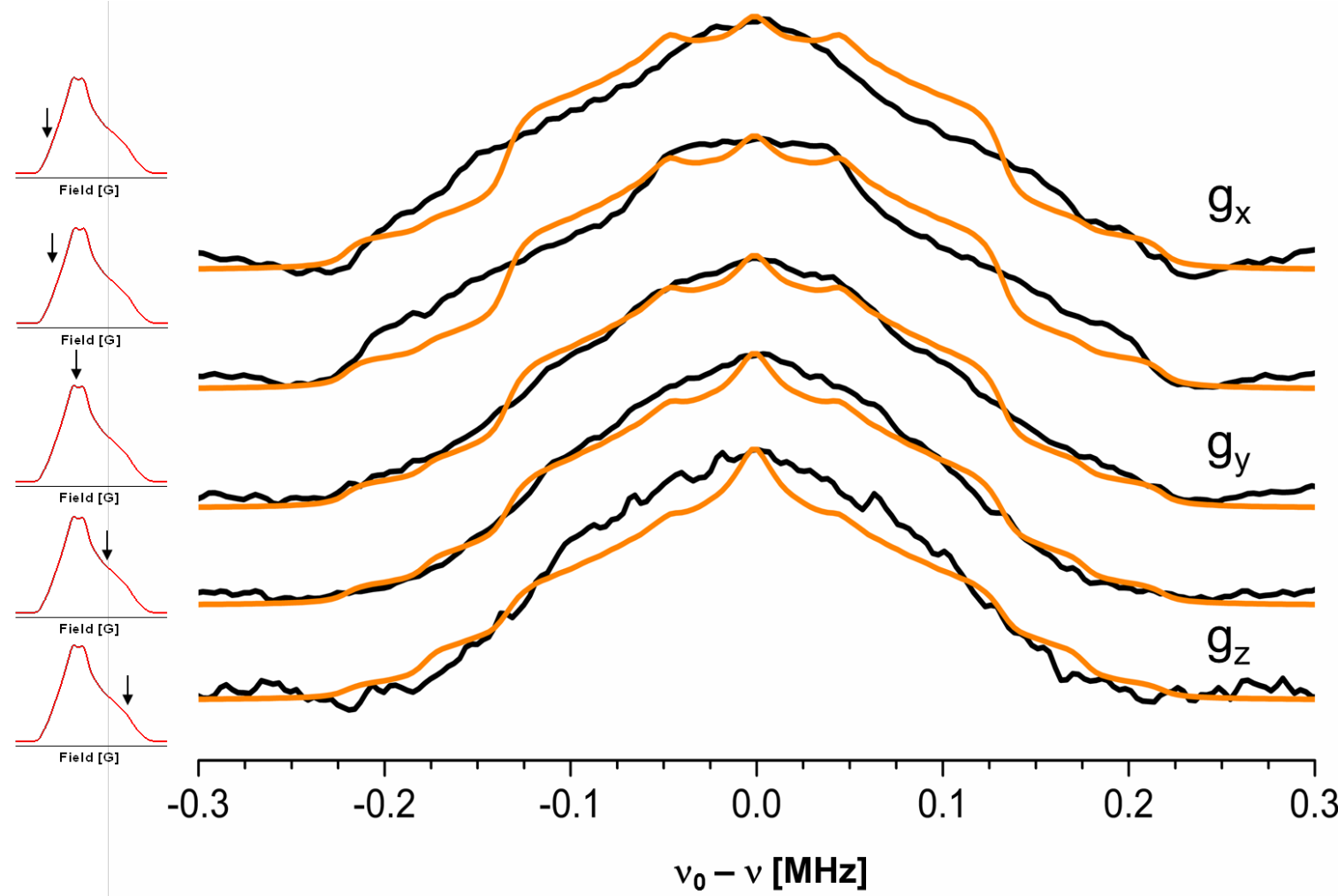

Figure 4-18. Orientation selective ${ }^{2} \mathrm{H}$ Mims ENDOR spectra of $\mathrm{NH}_{2} \mathrm{Y}_{731}{ }^{\circ}$ in $\mathrm{D}_{2} \mathrm{O}$ assay buffer of the weakly coupled region which is indicated in Figure 4-3. ENDOR spectra (in black) are recorded across the EPR line in $12 \mathrm{G}$ steps. On the left side the field positions at which the ENDOR experiment was recorded are indicated. The simulations (orange) were performed with the parameters from Table 4-4 for a deuteron of a $\mathrm{D}_{2} \mathrm{O}$ molecule. Conditions for all ENDOR spectra: $\mathrm{T}=10 \mathrm{~K}, \mathrm{MW}$ pulse $(\pi / 2)=40 \mathrm{~ns}, \tau=360 \mathrm{~ns}, \mathrm{RF}$ pulse $=40 \mu \mathrm{s}$, repetition time $=150 \mathrm{~ms}, 2000$ scans $/ \mathrm{spectrum}$, $40 \mathrm{~h} / \mathrm{spectrum}$. 
Orientation selective high-field ENDOR on $\alpha-\mathrm{ND}_{2} \mathrm{Y}_{731}{ }^{\circ}$ - coupling to external hydrogenbonds

As for $N_{2} \mathrm{Y}_{730}{ }^{\circ}$ we have recorded orientation selective high-field ENDOR spectra for $\mathrm{ND}_{2} \mathrm{Y}_{731}{ }^{\circ}$ in the spectral region within $\pm 1.5 \mathrm{MHz}$ where external hydrogen bonds are expected. Three ENDOR spectra were recorded across the EPR line in $18 \mathrm{G}$ steps (Figure 4-16), starting at $\mathrm{g}_{\mathrm{x}}$. Like in case of $\mathrm{ND}_{2} \mathrm{Y}_{730^{\circ}}$ (see Figure 4-11) we observed a pronounced orientation selection when going from $g_{y}$ to $g_{z}$. The strong intensity of the peaks at $\pm 0.6 \mathrm{MHz}$ at $g_{x}$ and $g_{y}$ suggested also here that they could constitute the perpendicular component of an axially symmetric hyperfine tensor $\left(A_{\perp}\right)$.

However from the DFT calculations on small model systems we learned, that the hyperfine tensor for deuterium does not possess the typical dipolar tensor form when the deuterium nucleus is close enough to the $\pi$-system of the $\mathrm{NH}_{2} \mathrm{Y}^{\circ}$. As the out-of-plane angle of the hydrogen-bond to the aromatic ring plane of $\mathrm{Y}_{731}$ is the same like in the case of $\mathrm{NH}_{2} \mathrm{Y}_{730}{ }^{\circ}$ $\left(45^{\circ}\right)$ (see Figure 4-14) we used the parameter set from Table 4-1 for $\operatorname{Tyr}_{731}$-OD as input parameters for $\mathrm{Tyr}_{730^{-}} \mathrm{OD}$ (assuming hereby that after structural minimization of $\mathrm{NH}_{2} \mathrm{Y}_{731}{ }^{\circ}$ in the DFT cluster model systems a similar hydrogen-bond conformation is present). The parameters were slightly changed for the $A_{y}$ and $Q_{3}$ value and are lower than for the calculated small model systems. Additionally we found out that we could use as a second hyperfine tensor, a tensor for a $\mathrm{D}_{2} \mathrm{O}$ molecule which is in a distance of $1.7-1.8 \AA$ in close contact to the $\pi$-plane of the aromatic system. Interestingly the $0^{\circ}-D$ distance vector is exactly orthogonal to the $\pi$-plane. 


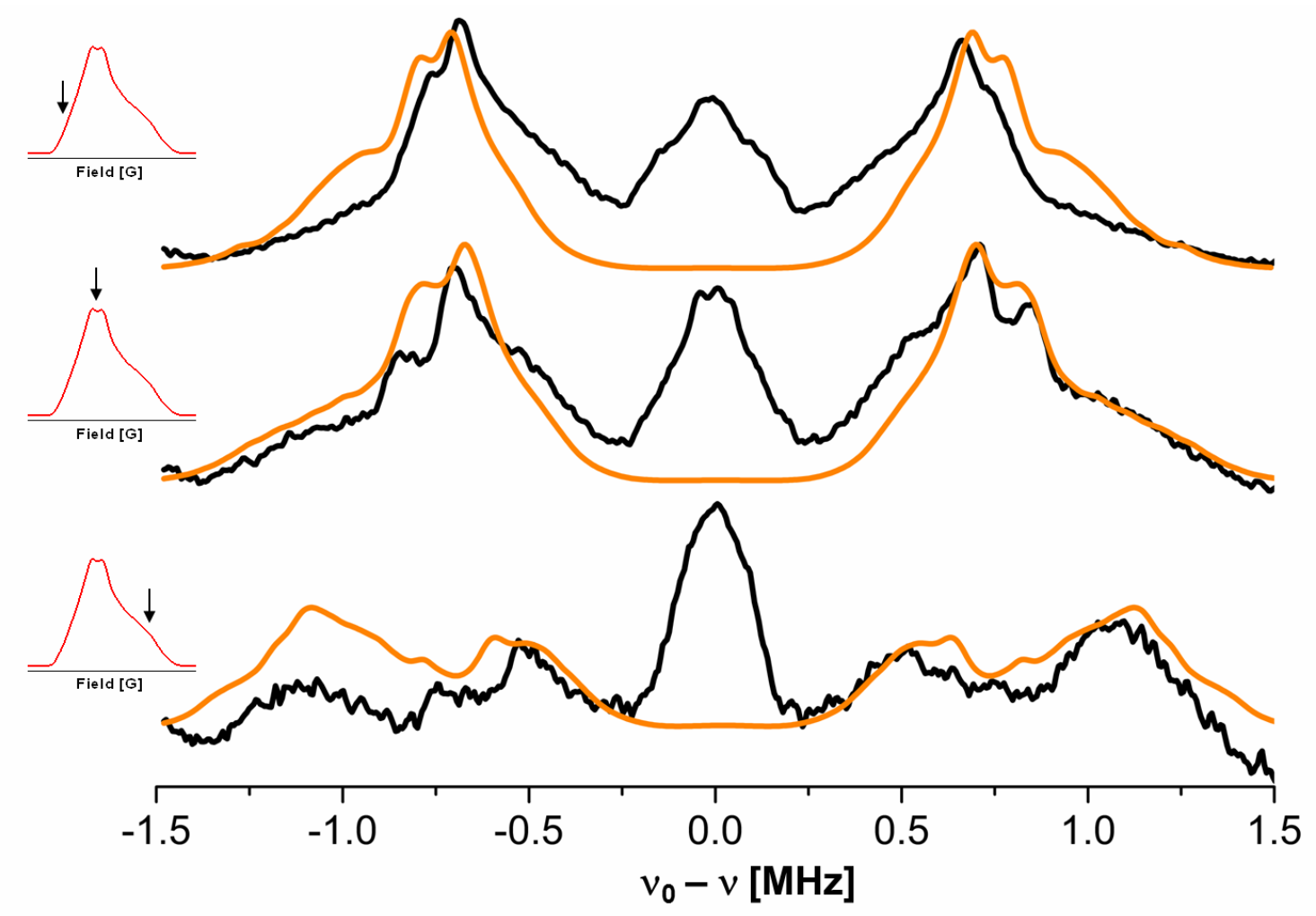

Figure 4-19. Orientation selective ${ }^{2} \mathrm{H}$ Mims ENDOR spectra of $\mathrm{NH}_{2} \mathrm{Y}_{731}{ }^{\bullet}$ in $\mathrm{D}_{2} \mathrm{O}$ assay buffer in the region $\pm 1.5 \mathrm{MHz}$ where external hydrogen bonds are expected. Spectra were recorded across the EPR line in $18 \mathrm{G}$ steps (black). On the left side the field positions at which the ENDOR experiment was performed are indicated. In the simulations the hyperfine and quadrupole parameters as well as Euler angles for the exchangeable hydrogens of the $\mathrm{NH}_{2}$-group were included (see Table 4-4). Conditions for all ENDOR spectra: $\mathrm{T}=10 \mathrm{~K}$, MW pulse $(\pi / 2)=20 \mathrm{~ns}, \tau=320 \mathrm{~ns}$, RF pulse $=40 \mu \mathrm{s}$, repetition time $=150 \mathrm{~ms}, 2500 \mathrm{scans} / \mathrm{spectrum}, 50 \mathrm{~h} / \mathrm{spectrum}$. 


\begin{tabular}{|c|c|c|c|c|c|c|c|c|c|c|}
\hline & $A_{x}$ & $A_{y}$ & $A_{z}$ & $\alpha$ & $\beta$ & $\gamma$ & $Q_{1}$ & $Q_{2}$ & $Q_{3}$ & $r_{O-D}$ \\
\hline $\mathbf{D}_{\mathbf{2}} \mathbf{O}(\mathbf{1})$ & & & & & & & & & & \\
\hline $\operatorname{Sim}$ & -0.09 & -0.09 & 0.17 & 0 & -20 & 30 & 0.12 & -0.06 & -0.06 & $3.5^{(\mathrm{a})}$ \\
\hline $\mathbf{D}_{\mathbf{2}} \mathbf{O}(\mathbf{2})$ & & & & & & & & & & \\
\hline $\operatorname{Sim}$ & -1.51 & -1.54 & 1.29 & 0 & 0 & 0 & -0.03 & -0.06 & 0.11 & $1.7-1.8^{(\mathrm{b})}$ \\
\hline $\mathbf{T y r}_{\mathbf{7 3 0}}-\mathbf{O D}(\mathbf{3})$ & & & & & & & & & & \\
\hline $\operatorname{Sim}$ & -2.38 & -1.15 & 1.15 & -14 & 133 & 78 & -0.03 & -0.06 & 0.03 & $1.8^{(b)}$ \\
\hline $\mathbf{N D}_{\mathbf{2}}$ (D2) & & & & & & & & & & \\
\hline $\operatorname{Sim}$ & 3.02 & 0.67 & 4.37 & 0 & 126 & -60 & 0.08 & -0.04 & -0.04 & - \\
\hline $\mathbf{N D}_{\mathbf{2}}$ (D1) & & & & & & & & & & \\
\hline $\operatorname{Sim}$ & 1.04 & 1.23 & 3.39 & 0 & 50 & 0 & 0.08 & -0.04 & -0.04 & - \\
\hline
\end{tabular}

Table 4-4. Parameter set for deuterons 1-3 which are depicted in Figure 4-17 around the 3-aminotyrosyl radical at position 731. The hyperfine and quadrupole values are given in $\mathrm{MHz}$ and the corresponding Euler angles $(\alpha, \beta, \gamma)$ are given in degrees. The conversion factor from ${ }^{1} \mathrm{H}$ to ${ }^{2} \mathrm{H}$ frequency is 6.5 with respect to Table 3-1. Distances are given in $\AA$ and errors are estimated approximately to be 5-10 \%. (a) from point-dipole model; (b) from DFT calculations on the angular and distance dependence of $A$ and $Q$ values of small model systems (data not shown). Like for the simulation of the spectra in Figure 4-11 the parameters of the $\mathrm{ND}_{2}$-group were included without any change.

Although the three possible hydrogen positions are not approved through a separate DFT cluster calculation on $\mathrm{NH}_{2} \mathrm{Y}_{731^{\circ}}$, the ENDOR simulations strongly suggest that all $\mathrm{O}^{\circ}-\mathrm{D}$ distance vectors are orthogonal or nearly orthogonal to the aromatic plane (see Figure 4-17). Especially the similar behaviour of the hyperfine and quadrupole tensors in the external hydrogen-bond region (compare ENDOR spectra of $\mathrm{ND}_{2} \mathrm{Y}_{730}{ }^{\circ}$ and $\mathrm{ND}_{2} \mathrm{Y}_{731}{ }^{\circ}$ in Figure 4-11 and 4-16) supports our finding that the hydrogen-bond network is very similar to the one around $\mathrm{NH}_{2} \mathrm{Y}_{730}{ }^{\circ}$ with the exception that we see additionally a second more distant water molecule residing near the $\alpha 2: \beta 2$ subunit interface. 


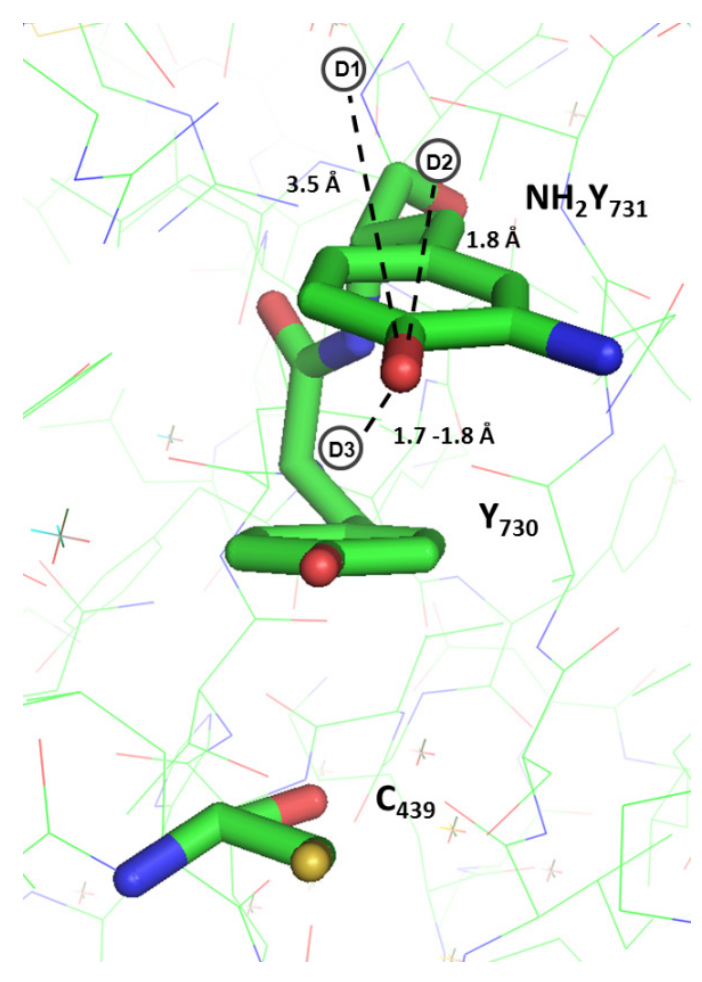

Figure 4-20. Crystal structure of $\mathrm{NH}_{2} \mathrm{Y}_{731}-\alpha 2$ and locations of deuterons obtained from the analysis of the orientation selective ENDOR spectra of $\alpha-\mathrm{NH}_{2} \mathrm{Y}_{731}{ }^{\circ}$ in $\mathrm{D}_{2} \mathrm{O}$ assay buffer. The directions of the $\mathrm{O}^{\circ}-\mathrm{D}$ distance vectors are indicated together with the distances from Table 4-4.

\subsection{Discussion}

In order to get structural insights about a putative hydrogen-bond network in the $Y_{731} \leftrightarrow$ $\mathrm{Y}_{730} \leftrightarrow \mathrm{C}_{439}$ triad of the $\alpha$-subunit of E.coli RNR during radical propagation, we have site-specifically incorporated at position 730 and 731,3 -aminotyrosine $\left(\mathrm{NH}_{2} \mathrm{Y}\right)$ using the in vivo nonsense suppressor tRNA/aminoacyl-tRNA synthetase methodology. $\mathrm{NH}_{2} \mathrm{Y}$ could serve hereby as a competitive spin trap in radical transport. First spectroscopic evidence for the formation of the 3-aminotyrosyl radical $\left(\mathrm{NH}_{2} \mathrm{Y}^{\circ}\right)$ in $\mathrm{NH}_{2} \mathrm{Y}_{730}-\alpha 2$ were reported earlier through X-band EPR and PELDOR spectroscopy as well as stopped-flow UV-vis kinetics (11). We have further characterized the electronic structure of the trapped radical at position 730 and 731 in detail as illustrated in Chapter 3 and have determined its g-values. The latter analysis suggested strong intermolecular hydrogen-bond interactions around $\mathrm{NH}_{2} \mathrm{Ys}^{\bullet}$ at both locations that could be essential for the PCET in the $\alpha$-subunit. Our high-field ENDOR result on $\mathrm{ND}_{2} \mathrm{Y}_{730^{\circ}}$ and $\mathrm{ND}_{2} \mathrm{Y}_{731}{ }^{\circ}$ gave direct evidence for three exchangeable protons with two 
being in direct hydrogen-bond distance to the oxygen of the $\mathrm{NH}_{2} \mathrm{Y}^{\bullet}$. For $\mathrm{ND}_{2} \mathrm{Y}_{730^{\circ}}$ one exchangeable proton is located at a distance of $2.6 \AA$ and the tensor points in the direction of a water molecule, which is also visible in crystal structures of wt- $\alpha 2$ and $\mathrm{NH}_{2} \mathrm{Y}_{730}-\alpha 2$ of E.coli RNR as well as in the $\alpha$-subunit of human $\operatorname{RNR}(3,12,13)$.

The location of the water molecule near $\mathrm{NH}_{2} \mathrm{Y}_{730}-\alpha 2$ during the active state suggests that it might have mechanistic functions during $\mathrm{H}$-atom transfer between $\mathrm{Y}_{731}, \mathrm{Y}_{730}$ and $\mathrm{C}_{439}$ as recent studies on electron transfer in peptides with relais-amino acids have pointed out. The importance of a water environment for a PCET between a $C^{\bullet}$ and a $Y$ which are separated by $10 \AA$ was demonstrated by Giese et al. . Here a peptide system was used which contains tyrosine at the $\mathrm{N}$-terminal end as an electron donor, a dialkoxyphenylalanine at the $\mathrm{C}$ terminal end as a precursor for the electron acceptor, and the relay amino acid cysteine on a half-way between donor and acceptor. The effect on radical transfer was demonstrated through an isotope effect of 2 when performing the reaction in $D_{2} \mathrm{O}$ suggesting hereby a water-mediated PCET. The yield of the generated $\mathrm{Y}^{\bullet}$ decreased from 15 to $7 \%$ (14).

The DFT cluster calculations on $\alpha-\mathrm{NH}_{2} \mathrm{Y}_{730}{ }^{\circ}$ predict an optimized structure in which the observed water molecule from the ENDOR experiments is in hydrogen bond distance of $2.2 \AA$ to the oxygen of $\mathrm{NH}_{2} \mathrm{Y}_{730^{\circ}}$. Furthermore DFT studies on larger systems which include the substrate GDP has additionally to be performed in order to understand the $\mathrm{H}$-atom transfer kinetics of the $Y_{731} \leftrightarrow Y_{730} \leftrightarrow C_{439}$ triad. The substrate is hereby one trigger probably together with the detected water molecule which can control PCET kinetics in the $\alpha$-subunit.

Interestingly, we note that the ENDOR data of the trapped intermediate show the hydrogenbond to the water molecule at a longer distance $(2.6 \AA)$ than obtained from the calculation on the structure of the resting $\alpha 2$-subunit $(2.2 \AA)$.

The longer distance is also reflected in the g-values, which are better reproduced by the DFT model without a water molecule suggesting no hydrogen-bond at $2.6 \AA$ distance (see Table 4-3). This difference is however intriguing and might suggest that small reorientations of this water molecule could influence the energetics of the radical transfer process.

In the resting (inactive) state the molecule might be close enough to slow down the radical transfer while in the active state the hydrogen-bond is loosened.

Furthermore, our ENDOR data are consistent with the couplings to exchangeable protons of $\mathrm{Y}_{731}$ and $\mathrm{C}_{439}$, as confirmed by DFT calculations. This is for the first time a combined 
experimental and theoretical evidence for the postulated hydrogen-bond network between the essential PCET pathway residues in the $\alpha 2$ subunit of E.coli RNR at position 730.

The water molecule which we observe in our ENDOR studies can most probably penetrate through a water accessible cleft between the $\mathrm{N}$-terminal domain and the $\alpha / \beta$ barrel where the PCET pathway residues $\mathrm{Y}_{731}, \mathrm{Y}_{730}$ and $\mathrm{C}_{439}$ are placed. All crystal structures show that it is already present before $\alpha 2 / \beta 2$ subunit interaction and is surrounded by polar amino acids like $Y_{413}, D_{334}$, and $T_{303}$ as well as the backbone carbonyl of $L_{438}$. To date a possible role of a water molecule in the PCET of the active E.coli RNR has not been discussed, likely due to the lack of structural data on the intermediate states. However, while a mechanism for PCET in form of an HAT mechanism between the two $Y_{731}$ and $Y_{730}$ seems to be widely accepted due to the vicinity, the $\pi$-stacking and the relative redox potentials of these two residues the PCET mechanism between $\mathrm{Y}_{730}$ and $\mathrm{C}_{439}$ is still not clear.

In order to rationalize the energetics for $\mathrm{H}$-atom transfer between $\mathrm{Y}_{731} \leftrightarrow \mathrm{Y}_{730} \leftrightarrow \mathrm{C}_{439}$ in the $\alpha$-subunit the redox potentials have been also computed against the reaction coordinates (Figure 4-18). The crystal structure of the $\alpha$-subunit with GDP and dTTP as substrate and effector has served as an input for the calculation. We found that the transition states as well as the redox potentials were different depending on the presence of a water molecule near $Y_{730}$ (residing in a close distance of $2.2 \AA$ ). When water is present the transition states for $\mathrm{H}$-atom transfer between $\mathrm{Y}_{731}$ and $\mathrm{Y}_{730}$ as well as $\mathrm{Y}_{730}$ and $\mathrm{C}_{439}$ are in both cases increased by 1.1 and $1.3 \mathrm{kcal} / \mathrm{mol}$, respectively. The increased barriers significantly slow down the kinetics of $\mathrm{H}$-atom transfer by about an order of magnitude. The longer distance of $2.6 \AA$ based on our ENDOR data resembles hereby the calculation without the water molecule. 


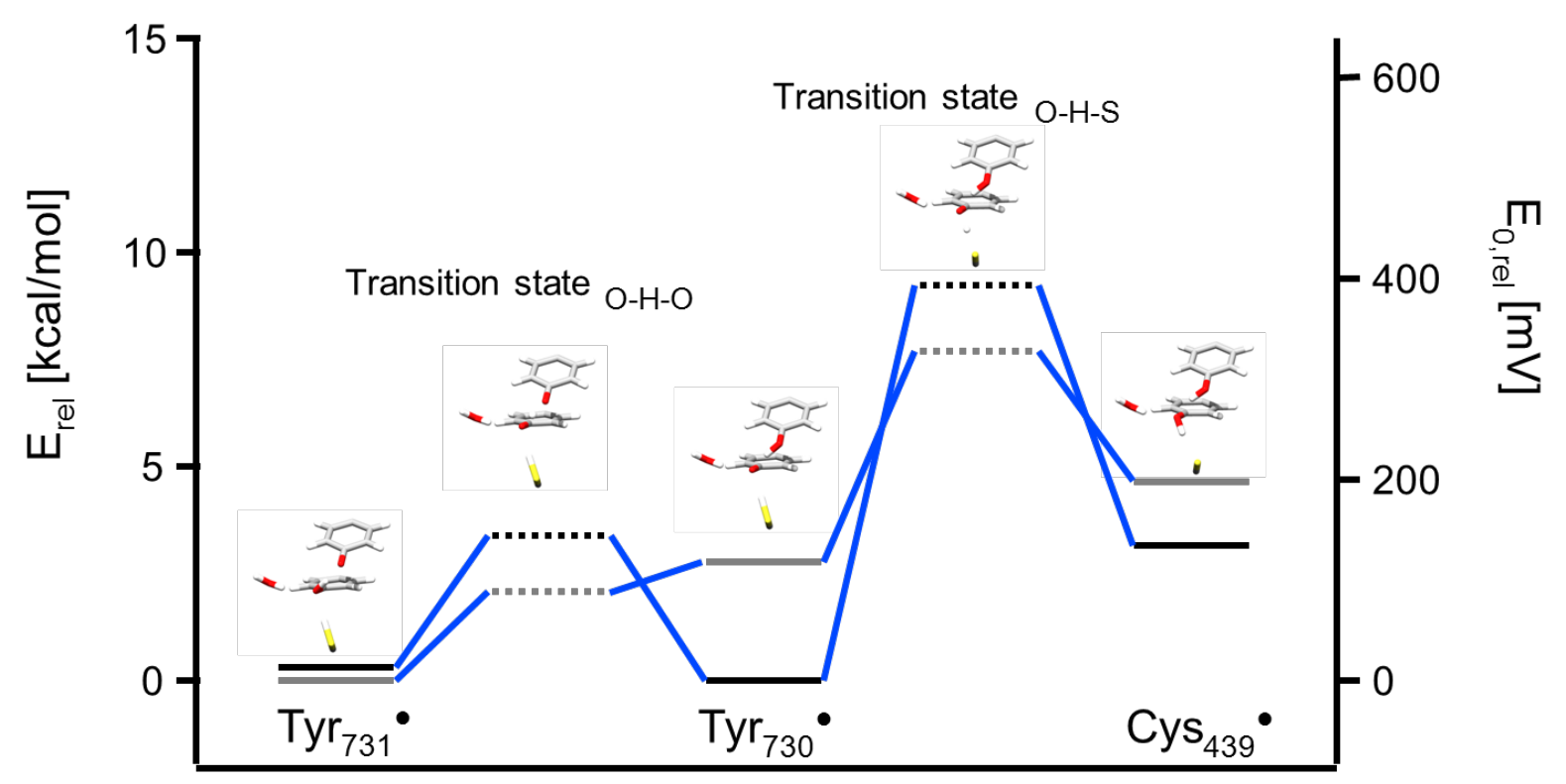

reaction coordinate

Figure 4-21. Energy level diagram including the transition states for the PCET pathway residues $\mathrm{Y}_{731}-\mathrm{Y}_{730}-\mathrm{C}_{439}$ in the $\alpha$-subunit. The energy levels and transition states shown in grey are computed without the water molecule placed nearby $Y_{730}$. In black the computation with the water near $Y_{730}$ is shown. Without the water molecule the reduction potentials for $\operatorname{Tyr}_{730^{\circ}}$ and $\mathrm{Cys}_{439}{ }^{\circ}$ are raised by 2.8 and $1.4 \mathrm{kcal} / \mathrm{mol}$ whereas for $\mathrm{Tyr}_{731}$ they remain nearly the same.

For $\alpha-\mathrm{NH}_{2} \mathrm{Y}_{731}$, ENDOR spectroscopy in combination with already obtained results from DFT studies on $\alpha-\mathrm{NH}_{2} \mathrm{Y}_{730}{ }^{\circ}$ suggest a very similar hydrogen-bond network around $\alpha-\mathrm{NH}_{2} \mathrm{Y}_{731}$. Furthermore the g-values of $\alpha-\mathrm{NH}_{2} \mathrm{Y}_{731}{ }^{\bullet}$ are the same like for $\alpha-\mathrm{NH}_{2} \mathrm{Y}_{730}{ }^{\bullet}\left(g_{x}=2.0052\right.$, $\left.g_{y}=2.0042, g_{z}=2.0022\right)$ and give further evidence for the similarity of hydrogen-bond networks.

Still it is not known which could be the second hydrogen-bond partner besides $Y_{730}$ in case of $\alpha-\mathrm{NH}_{2} \mathrm{Y}_{731}$ ' which should be located near the $\alpha / \beta$ subunit interface. Orientation selective ENDOR spectra could be successfully simulated assuming one water molecule which is positioned with a $\mathrm{D}^{\circ} \mathrm{O}^{\bullet}$ distance of $1.8 \AA$ orthogonal to the aromatic plane. Another possibility would be $Y_{356}$ of the $\beta$-subunit as the PCET partner. Further we notice that $Y_{731}$ or $\mathrm{NH}_{2} \mathrm{Y}_{731}$ is highly flexible and that during conformational change (forward and backward PCET) the second hydrogen-bond partner could possible interchange at the $\alpha / \beta$ subunit interface. 
The influence of water or water structures on interprotein electron-transfers has been theoretically discussed with the result that across aqueous interfaces strongly coupled tunneling pathways involve single or several water molecules which form a mediating water structure in order to facilitate electron transfer at subunit interfaces (15). More recent results on the redox reaction between methylamine dehydrogenase (MADH) and amicyanin from Paracoccus denitrificans report a "molecular break-water", which functions as an organizing unit for solvent-exposed residues hereby assisting an enhanced electron transfer between these two proteins (16).

In our case a water structure at the subunit interface is further supported through the observation that a second water molecule is visible in the orientation selective ENDOR spectrum residing at the $\alpha / \beta$ interface which is positioned nearly perpendicular to the aromatic plane. This second water molecule is also visible in the $\mathrm{NH}_{2} \mathrm{Y}_{731}-\alpha 2 \mathrm{X}$-ray structure $\left(0^{\circ}\right.$ - O distance of $\left.4.9 \AA\right)$.

In conclusion we could characterize the hydrogen-bond network around $\mathrm{NH}_{2} \mathrm{Y}_{730}$ and $\mathrm{NH}_{2} \mathrm{Y}_{731}{ }^{\circ}$ in the $\alpha$-subunit during the active state of the E.coli RNR enzyme through a high-field ENDOR/DFT approach. The finding suggests hereby that the PCET mechanism at both positions is a co-linear one. Interesting is also that through high-field ENDOR spectroscopy detected water molecules near $\mathrm{NH}_{2} \mathrm{Y}_{730 / 731}{ }^{\circ}$ could have mechanistic influence on the PCET process. They have the ability either to accelerate or to slow down the H-atom transfer depending on their position with respect to the $\mathrm{NH}_{2} \mathrm{Ys}^{\bullet}$ in the $\alpha$-subunit. The here examined ENDOR spectra of the 3-aminotyrosyl radical at location 730 and 731 showed complicated but unique hyperfine spectra originating from several deuterium nuclei which could be interpreted through a sophisticated simulation strategy of high-field EPR and ENDOR spectra and through the usage of quantum chemical calculations which facilitated the choice of different parameter sets for the ENDOR simulation and could unambiguously interpret the g-values of $\alpha-\mathrm{NH}_{2} \mathrm{Y}_{730 / 731}$. 
Materials and methods of chapter 3 and 4 


\section{Materials and Methods}

\section{Materials}

$\mathrm{D}_{2} \mathrm{O}$ (99.8 atom \% in D), Minicon concentration device (YM-30), Eppendorf tubes, CDP, ATP, X-band ER 221TUB/4 Bruker (ID = $4 \mathrm{~mm}$ ), Q-band sample tubes ER 221TUB-Q10 Bruker (ID = $1 \mathrm{~mm}$ ), W-band sample tubes E600-213 Bruker (ID = $0.5 \mathrm{~mm}$ ), round quartz capillary tubes from Wilmad (ID = 0.3), HEPES, $\mathrm{MgSO}_{4}$, EDTA.

\section{Preparation of high-field EPR samples}

Prereduced $\mathrm{NH}_{2} \mathrm{Y}_{730 / 731}-\alpha 2$ and $\beta 2$ were combined in an equimolar ratio and concentrated at $4{ }^{\circ} \mathrm{C}$ in a Minicon concentration device (YM-30 membrane) to a final complex concentration of $\left.100 \mu \mathrm{M}\left(\varepsilon_{280} \mathrm{~nm}(\alpha 2+\beta 2)\right)=320 \mathrm{mM}^{-1} \mathrm{~cm}^{-1}\right)$ in $\mathrm{D}_{2} \mathrm{O} / \mathrm{H}_{2} \mathrm{O}$ buffer consisting $50 \mathrm{mM}$ Hepes, $15 \mathrm{mM} \mathrm{MgSO}_{4}$ and $1 \mathrm{mM}$ EDTA (assay buffer) at a pD of 8.0. The concentrated samples were divided into $100 \mu \mathrm{L}$ aliquots, placed in $1.5 \mathrm{~mL}$ Eppendorf tubes, and flash-frozen in liquid $\mathrm{N}_{2}$. $\mathrm{NH}_{2} \mathrm{Y}_{356}-\beta 2$ and prereduced $\alpha 2$ were already prepared in both buffer media in $100 \mu \mathrm{L}$ aliquots by Ellen Minnihan from the Stubbe group at MIT and sent to the MPIBPC for highfield EPR analysis. The high-field EPR samples were then prepared by thawing each aliquot on ice and adding CDP and ATP to final concentrations of 2 and $6 \mathrm{mM}$ in a $20 \mu \mathrm{L}$ sample volume. Each reaction was allowed to proceed for $~ 30 \mathrm{~s}$ at room temperature and was then quenched in an EPR tube by hand-freezing with liquid $\mathrm{N}_{2}$. The reaction in $\mathrm{D}_{2} \mathrm{O}$ was carried out after exchanging the $\mathrm{H}_{2} \mathrm{O}$ buffer of prereduced $\mathrm{NH}_{2} \mathrm{Y}_{730 / 731^{-}} \alpha 2$ and $\beta 2$ through deuterated assay buffer by 5 concentration/dilution cycles at $4{ }^{\circ} \mathrm{C}$ using a Minicon concentration device and a YM-30 membrane until the solution consisted of $>99 \%$ deuterated buffer. Nucleotide stock solutions were also prepared in deuterated buffer.

\section{Electron spin echo spectroscopy at $9,34,94$, and $180 \mathrm{GHz}$}

9, 34 and $94 \mathrm{GHz}$ pulsed EPR spectra were recorded on Bruker Elexsys spectrometers of the series E580 and E680 using a $\pi / 2-\pi$ spin echo sequence with typical $\pi / 2$ pulse lengths of 32 ns. The $180 \mathrm{GHz} \mathrm{NH} \mathrm{Y}_{730^{\circ}}$ pulsed EPR spectrum was recorded on a home built spectrometer in Frankfurt am Main by Prof. Marina Bennati previously reported with $\pi / 2$ pulse lengths of $32 \mathrm{~ns}$ (1). Other experimental details are given in the figure captions. The pulsed EPR spectra 
were recorded at $70 \mathrm{~K}$, where $\mathrm{Y}_{122}{ }^{\bullet}$ is not detectable as it relaxes more rapidly than a $\mathrm{NH}_{2} \mathrm{Y}^{\bullet}$ due to its vicinity to the di-iron cluster (2). At this temperature, the electron spin echo (ESE) spectra at all four frequencies contain only contributions from the $\mathrm{NH}_{2} \mathrm{Y}_{730}$ signal.

\section{Simulation of EPR spectra at 9, 34 and $94 \mathrm{GHz}$}

The EPR powder spectra were analyzed using SimFonia software (Bruker) and in case of Xband EPR spectra additionally the EasySpin software was used which is free available (3). In

order to independently determine the optimal number of parameters, g-values were obtained from the $180 \mathrm{GHz}$ EPR spectrum and the majority of the hyperfine couplings were fixed by iterative simulations of the ${ }^{14} \mathrm{~N}$ spectra in $\mathrm{D}_{2} \mathrm{O}$ at the two different frequencies 9 and $94 \mathrm{GHz}$. At the end the optimized parameters obtained from the $\mathrm{NH}_{2} \mathrm{Y}_{730^{\circ}}$ simulations in $\mathrm{D}_{2} \mathrm{O}$ were used as input for the simulations of the spectra in $\mathrm{H}_{2} \mathrm{O}$. The latter spectra contained additionally the contribution of the two exchangeable protons of the $\mathrm{NH}_{2}$ group. The simulation of the amino proton contribution was complex because of their large hyperfine anisotropy and the non co-linearity of the g- and A-axes for these protons. The simulation thus required the introduction of the three Euler angles between the g- and A-axes as additional parameters.

\section{$34 \mathrm{GHz}$ Davies ENDOR on $\alpha-\mathrm{NH}_{2} \mathrm{Y}_{730}{ }^{\circ}$ and $\alpha-\mathrm{NH}_{2} \mathrm{Y}_{731}{ }^{\circ}$ in $\mathrm{D}_{2} \mathrm{O}$ assay buffer}

$34 \mathrm{GHz}{ }^{1} \mathrm{H}$ ENDOR measurements were performed on the Bruker Elexsys spectrometer of the series E580 and were employed with the Davies ENDOR sequence $(\pi-\operatorname{RF}-\pi / 2-\tau-\pi-\tau-$ echo) at $70 \mathrm{~K}$ in deuterated buffer in order to probe for large proton methylene couplings which should be distinguishable from small ring proton couplings. At $70 \mathrm{~K}$ the strong signal of $\operatorname{Tyr}_{122}{ }^{\bullet}$ is not visible and the ENDOR experiment is performed only on the $\mathrm{ND}_{2} \mathrm{Y}_{730}{ }^{\circ}$ or $\mathrm{ND}_{2} \mathrm{Y}_{731} \cdot$ signal. The length of the preparation pulse (first $\pi$ pulse), the $\pi / 2$ and RF pulses are described in the figure captions of the results section. 


\section{4 and $94 \mathrm{GHz}$ Mims ENDOR spectroscopy on $\alpha-\mathrm{NH}_{2} \mathrm{Y}_{730}{ }^{\circ}$ and $\alpha-\mathrm{NH}_{2} \mathrm{Y}_{731}{ }^{\circ}$ in $\mathrm{D}_{2} \mathrm{O}$ buffer}

${ }^{2} \mathrm{H}$ ENDOR measurements were employed with the Mims ENDOR sequence $(\pi / 2-\tau-\pi / 2-\mathrm{RF}$ $-\pi / 2-\tau$ - echo) at $10 \mathrm{~K}$. As the $\mathrm{Y}_{122}{ }^{\circ}$ in E.coli RNR does not possess exchangeable protons we could optimize our ENDOR performance for $\mathrm{NH}_{2} \mathrm{Y}_{730} 0^{\circ}$ and $\mathrm{NH}_{2} \mathrm{Y}_{731}$ by taking advantage of enhanced Boltzmann population difference at $10 \mathrm{~K}$ (4). Orientation selective ENDOR which focused on different spectral regions of the spin probe was the method of choice for the characterization of the $\mathrm{H}$-bond network during PCET in the $\alpha$-subunit at position 730 and 731. Different $\tau$ and $\pi / 2$ values were used in the Mims sequence as it is described in the figure captions of the results section.

\section{Simulation of ENDOR spectra}

$94 \mathrm{GHz}{ }^{2} \mathrm{H}$ ENDOR spectra were simulated using a MATLAB routine developed in-house that is based on a first order Hamiltonian (high-field condition) for the hyperfine and quadrupolar interaction. Previous EPR simulations of $\mathrm{NH}_{2} \mathrm{Y}_{730}{ }^{\circ}$ in $\mathrm{D}_{2} \mathrm{O}$ and $\mathrm{H}_{2} \mathrm{O}$ buffer resulted in a set of hyperfine tensor principal values $\left(A_{x x}, A_{y y}, A_{z z}\right)$ and Euler angles $(\alpha, \beta, \gamma)$. The $\mathrm{NH}_{2} \mathrm{Y}_{730^{\circ}}$ parameter set included four nuclei and the simulations were obtained at two different frequencies ( 9 and $94 \mathrm{GHz}$ ) with two different simulation software (SimFonia and EasySpin) (5). The hyperfine parameters for the two protons of the amino group were divided by a factor of 6.5 and were used together with the Euler angles as starting parameters for the simulation of the $94 \mathrm{GHz}^{2} \mathrm{H}$ ENDOR spectrum of $\mathrm{ND}_{2} \mathrm{Y}_{730^{\circ}}$ and $\mathrm{ND}_{2} \mathrm{Y}_{731} 1^{\circ}$. Importantly the Euler angles were not changed at all and the change of the hyperfine parameters is explained in the results section.

\section{DFT calculations}

DFT calculations on cluster model and small model systems were performed by Christoph Riplinger from the Neese group at the University of Bonn with the $a b$ initio, DFT and semiempirical SCF-MO (self-consistent field molecular orbital) package ORCA.

\section{Cluster model systems:}

The cluster models were generated from the crystal structure of the $\mathrm{NH}_{2} \mathrm{Y}_{730^{-}} \alpha 2$ mutant of E.coli RNR. Models 1 to 6 incorporated, besides the residues $\mathrm{NH}_{2} \mathrm{Y}_{730^{\circ}}, \mathrm{Y}_{731}$ and $\mathrm{C}_{439}$ that are directly involved in the PCET process, the surrounding residues $D_{334}, R_{411}, Y_{413}, S_{694}, A_{695}$ and 
$\mathrm{N}_{696}$. Models 1 and 5 additionally incorporated the water molecule WAT1 (see Figure 4-12) which is present between $\mathrm{NH}_{2} \mathrm{Y}_{730^{\circ}}$ and $\mathrm{D}_{334}$ in all three molecules of the asymmetric unit of the crystal structure. Models $2-4$ contain, additionally to WAT1, a second water molecule that is present in only two out of the three molecules in the crystal structure (see Figure 4-12). It is located between $\mathrm{NH}_{2} \mathrm{Y}_{730}$ and residing near $\mathrm{A}_{695}$. The side chains of the residues were always fully incorporated. Bonds were truncated in the backbone region between $C_{\alpha}$ and $\mathrm{C}(\mathrm{C}=\mathrm{O})$ and between $\mathrm{C}_{\alpha}$ and $\mathrm{N}(\mathrm{N}-\mathrm{H})$, respectively, and replaced by a $\mathrm{C}-\mathrm{H}$ bond. If the residues are in neighbour position or interrupted by one amino acid in the model structure (as for $\mathrm{S}_{694}-\mathrm{N}_{696}$ and $\mathrm{NH}_{2} \mathrm{Y}_{730^{\circ}}-\mathrm{Y}_{731}$ ) the respective backbone bonds were not cut. In order to mimic the steric influence of the protein in the native enzyme structure Cartesian constraints were included in the geometry optimization of the model structures. For all residues the coordinates of the $\mathrm{C}_{\alpha}$ and $\mathrm{C}_{\beta}$ atoms were kept fixed.

\section{Small model systems:}

All small model systems were constructed in order to analyze the influence of $Y_{731}, C_{439}$ and $\mathrm{H}_{2} \mathrm{O}$ on the g-values of $\mathrm{NH}_{2} \mathrm{Y}_{730}{ }^{\circ}$ (see Table 4-2). The simplest model are the 4-methyl-phenol and 2-amino-4-methylphenol radical (2-AMPR) which represents $\mathrm{NH}_{2} \mathrm{Y}_{730^{\circ}}$. As a non-H-bond equivalent (no intramolecular $\mathrm{H}$-bond is present) the 2-aminodimethyl-4-methyl-phenol radical has been used as a system. The intermolecular hydrogen-bond environment has then been gradually increased with $\mathrm{Y}_{731}, \mathrm{C}_{439}$ and $\mathrm{H}_{2} \mathrm{O}$, what gives rise to four additional models: 2-AMPR and $C_{439}, 2-A M P R$ and $Y_{731}, 2-A M P R, Y_{731}$ and $C_{439}$ and 2-AMPR, $Y_{731}, C_{439}$ and $\mathrm{H}_{2} \mathrm{O}$. In order to preserve the orientation of the hydrogen-bonds as present in the protein structure, angle and dihedral constraints were imposed during geometry optimization of the model systems.

\section{QM methodology:}

The optimizations were performed with unrestricted RI-BP using the def2-TZVP basis set in accordance with the def2-TZVP/J auxiliary basis set (6-9). Single-point energy calculations were carried out with RIJCOSX-B3LYP (10-12) using the def2-TZVPP basis set in accordance with def2-TZVPP/J as auxiliary basis set for all atoms. The EPR calculations were carried out using RIJCOSX-B3LYP using the EPR-II basis set (IGLO-II for sulfur) in accordance with def2TZVPP/JK as auxiliary basis set for all atoms (13-15). Grimme's 2006 semiempirical dispersion 
correction (keyword VDW06 in ORCA) was included to account for dispersion effects (16). Numerical frequency calculations were carried out at the same level of theory as for the optimization. From these frequency calculations the zero point energies (ZPE) for the relative energies were derived.

\section{PCET barrier calculations:}

For the calculation of the PCET barriers the crystal structure of $\alpha 2$ with GDP/dTTP as substrate and effector has been used (PDB ID $=4 R 1 R$ ). Relaxed surface scans were carried out pulling the hydrogen atom from $\mathrm{Y}_{731}$ or $\mathrm{C}_{439}$ to $\mathrm{Y}_{730^{\circ}}$. Transition state optimizations were performed using the P-RFO algorithm with an approximate Hessian matrix as initial Hessian as implemented in ORCA (17-18).

\section{Molecular visualization}

Visualization of the $\alpha 2$ crystal structure as well as modeling of the 3-aminotyrosyl radical into the $\alpha$-subunit was performed with PyMOL version 0.99 . 
Summary 


\section{Summary}

In this Thesis one of the most important enzymes in nucleotide metabolism - ribonucleotide reductase from the E.coli organism was examined through the usage of high-frequency/highfield EPR and ENDOR spectroscopy. The investigation focused on the structure of the proposed proton-coupled electron transfer (PCET) pathway which allows for radical propagation over a distance of $35 \AA$ from the $\beta$-subunit (the radical generation side) to the $\alpha$-subunit (the nucleotide turnover side). Three tyrosine residues are involved for efficient radical propagation on the pathway, $\operatorname{Tyr}_{356}$ in the $\beta$-subunit and $\operatorname{Tyr}_{731}$ and $\operatorname{Tyr}_{730}$ in the $\alpha$ subunit. In order to find evidence for the prerequisites of a proposed proton-coupled electron transfer at each tyrosine location, 3-aminotyrosine $\left(\mathrm{NH}_{2} \mathrm{Y}\right)$ has been incorporated through the in vivo suppressor tRNA/aminoacyl-tRNA synthetase methodology. After initiation of radical propagation through substrate and effector, $\mathrm{NH}_{2} \mathrm{Y}$ was able to function as a thermodynamic trap due to its lower redox potential of $190 \mathrm{mV}$ in comparison to Tyr. We have characterized for the first time the new amino acid radical at all three positions on the pathway through the application of multifrequency EPR spectroscopy. The information of this analysis paved the way to determine the hydrogen-bond network around $\mathrm{NH}_{2} \mathrm{Y}_{730}{ }^{\circ}$ and $\mathrm{NH}_{2} \mathrm{Y}_{731}$ in the $\alpha$-subunit through the usage of high-field ENDOR spectroscopy in conjunction with DFT calculations and to understand the energetics of the PCET mechanism at each location. Water structures were also detected around $\mathrm{NH}_{2} \mathrm{Y}_{730}{ }^{\circ}$ and $\mathrm{NH}_{2} \mathrm{Y}_{731}{ }^{\circ}$ which might be required for PCET processes in the $\alpha$-subunit.

Chapter 1 gives a general overview about different classes of ribonucleotide reductases (RNRs) with the E.coli class la being the prototype one. The E.coli RNR and its proposed radical propagation pathway are explained in more detail and an introduction to electron transfer theory as well as proton-coupled electron transfer theory is given. The latter is proposed to be important at each step of radical transfer on the radical propagation pathway. The three methods which were successively used to probe for a PCET event are: The tRNA/aminoacyl-tRNA synthetase methodology which can now be applied routinely in order to incorporate unnatural amino acids in E.coli RNR; secondly, multifrequency EPR spectroscopy which is a powerful approach for characterizing new amino acid radicals and, 
lastly, high frequency EPR spectroscopy combined with the double-resonance technique ENDOR, which is used for the characterization of hydrogen-bond networks around incorporated amino acid radical spin traps.

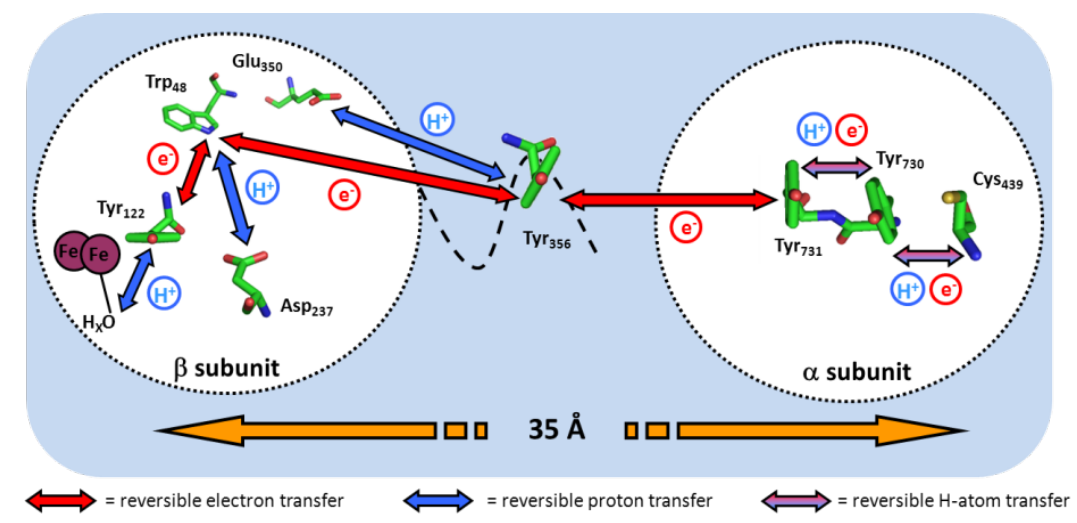

In Chapter $\mathbf{2}$ it is explained how the right tRNA/tRNA synthetase pair was selected for the incorporation of $\mathrm{NH}_{2} \mathrm{Ys}$ into the $\alpha$-subunit at location 730 and 731 . The expressions and purifications of $\mathrm{NH}_{2} \mathrm{Y}_{730}-\alpha 2$ and $\mathrm{NH}_{2} \mathrm{Y}_{731}-\alpha 2$ are described in detail and were all successful. On average $11.6 \mathrm{~g}$ of cell paste was obtained during the expression procedure resulting in approximately $125 \mathrm{mg}$ quantities of each $\mathrm{NH}_{2} \mathrm{Y}-\alpha 2$. During the purification procedure of $\mathrm{NH}_{2} \mathrm{Y}-\alpha 2 \mathrm{~s}$ one step could be skipped with respect to the purification of wt $\alpha 2$ in order to increase the yields. The incorporation of $\mathrm{NH}_{2} \mathrm{Ys}$ at each residue position (730 and 731) in the $\alpha$-subunit could be performed with high fidelity and with reasonable amounts of mutant proteins through the in vivo methodology.

1

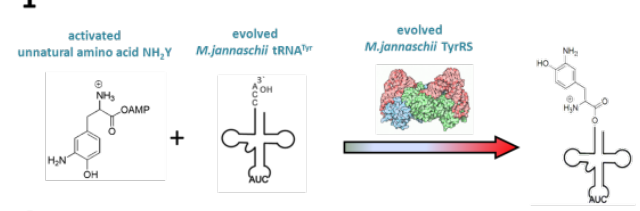

2

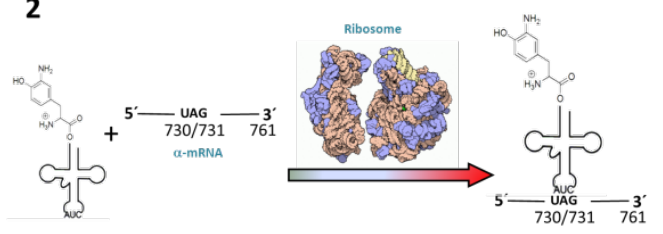

3

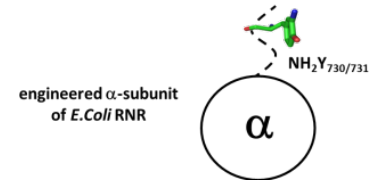


Chapter 3 demonstrates the investigation of $\alpha-\mathrm{NH}_{2} \mathrm{Y}_{730}{ }^{\circ}$ through multifrequency EPR spectroscopy at 9,94 and $180 \mathrm{GHz}$ which results in a first characterization of the electronic and molecular structure of a 3-aminotyrosyl radical in an enzyme. The $180 \mathrm{GHz}$ spectra are used in the analysis for a precise determination of the g-values, whereas the analysis of the 9 and $94 \mathrm{GHz}$ spectra in $\mathrm{D}_{2} \mathrm{O}$ and $\mathrm{H}_{2} \mathrm{O}$ buffer reveal the hyperfine tensors and Euler angles for the $\mathrm{NH}_{2}$-group nuclei and the $\mathrm{C}_{\beta}$-methylen protons. $34 \mathrm{GHz}$ ENDOR spectra support further the assignment of $C_{\beta}$-methylen proton hyperfine couplings, which could be used in order to determine the dihedral angle of the $C_{\alpha}-C_{\beta}$-bond with respect to the aromatic ring plane.
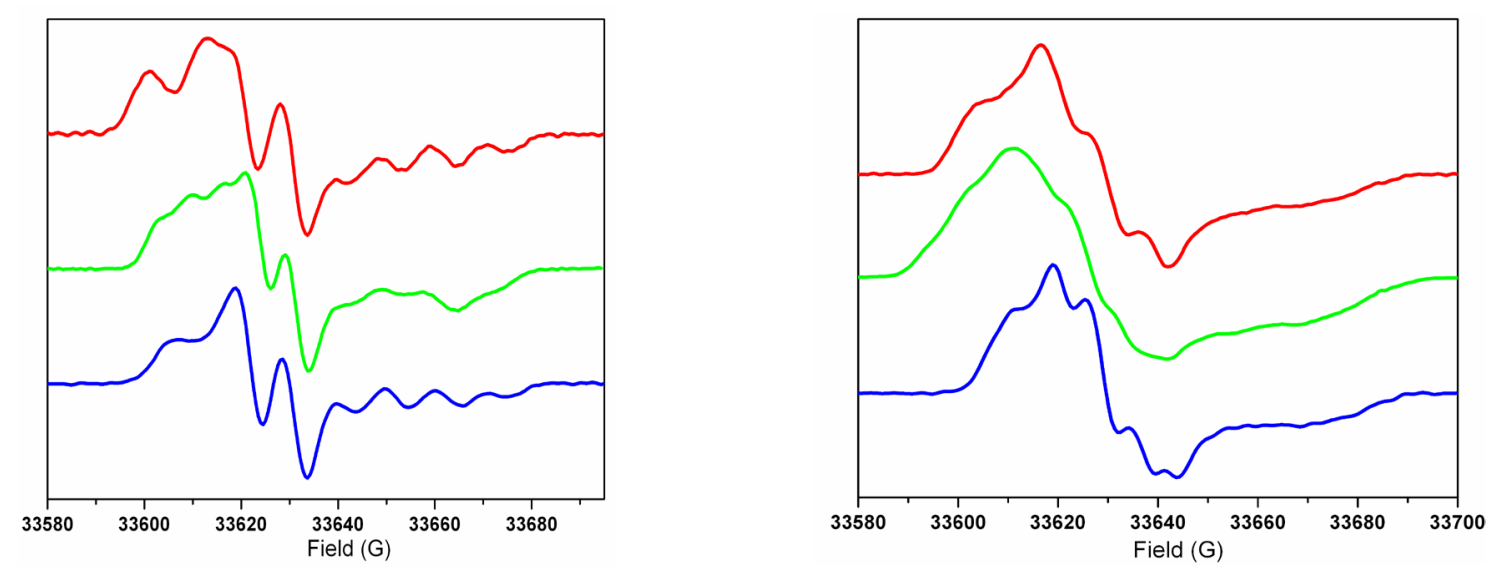

The obtained simulation parameter set for $\alpha-\mathrm{NH}_{2} \mathrm{Y}_{730} \cdot$ (six parameters per nucleus) was used as a reference for the simulation of $\alpha-\mathrm{NH}_{2} \mathrm{Y}_{731}{ }^{\bullet}$ and $\beta-\mathrm{NH}_{2} \mathrm{Y}_{356} 6^{\circ}$. The analysis of all three $\mathrm{NH}_{2} \mathrm{Y}^{\bullet} \mathrm{S}$ on the radical propagation pathway resulted hereby in different conformations of the $\mathrm{C}_{\alpha}-\mathrm{C}_{\beta}$-bond. Most surprisingly the $\mathrm{NH}_{2}$-group adopts the same conformation for all three $\mathrm{NH}_{2} \mathrm{Y}^{\circ} \mathrm{S}$ suggesting an intrinsic structural feature of the $\mathrm{NH}_{2} \mathrm{Y}$-probe during radical propagation. The Euler rotation exemplified for the $\mathrm{NH}_{2}$-group of $\mathrm{NH}_{2} \mathrm{Y}_{730}$ results in out-ofplane positions of the two $\mathrm{N}-\mathrm{H}$ bonds and the $\mathrm{C}-\mathrm{N}$ bond. With these structural informations in hand the $\mathrm{NH}_{2}$-group and the $\mathrm{C}_{\alpha}-\mathrm{C}_{\beta}$-bond could be modelled into the crystal structure at position 730 . The dihedral angle obtained from the EPR analysis differed only by $10^{\circ}$ from the one obtained from the wt crystal structure indicating that a substitution of $Y_{730}$ does not perturb the structure at this position. In case of $\alpha-\mathrm{NH}_{2} \mathrm{Y}_{731}{ }^{\circ}$ we obtain a dihedral angle which is in agreement with a $\pi$-stacked conformation between $\mathrm{NH}_{2} \mathrm{Y}_{731}{ }^{\circ}$ and $\mathrm{Y}_{730}$. However we notice that a second conformation due to the high flexibility at residue position 731 is 
possible. For $\mathrm{NH}_{2} \mathrm{Y}$ at position $\beta-356$ the obtained dihedral angle is a new structural restraint which could not be obtained to date from X-ray crystallographic studies on the $\beta$-subunit.

Chapter 4 focuses on the detailed characterization of the hydrogen-bond network around $\alpha-\mathrm{NH}_{2} \mathrm{Y}_{730}{ }^{\bullet}$ and $\alpha-\mathrm{NH}_{2} \mathrm{Y}_{731}{ }^{\bullet}$ during activity of E.coli ribonucleotide reductase. In $\mathrm{D}_{2} \mathrm{O}$ buffer one can detect all exchangeable hydrogens of functional groups in a radius of $5 \AA$ through ${ }^{2} \mathrm{H}$ Mims ENDOR spectroscopy. The orientation selective study most importantly revealed the mutual orientation of the hyperfine (dipolar) tensor with respect to the g-tensor and consequently the directions of $\mathrm{O}^{\circ}$-D distance vectors through interpretation of the Euler angles. Several orientation selective experiments covered hereby different ENDOR spectral regions of $\alpha-N_{2} \mathrm{Y}_{730}{ }^{\circ}$ and $\alpha-N D_{2} \mathrm{Y}_{731}{ }^{\circ}$ in order to systematically screen for different sorts of deuterons (i. e. deuterons from the $\mathrm{ND}_{2}$ group).

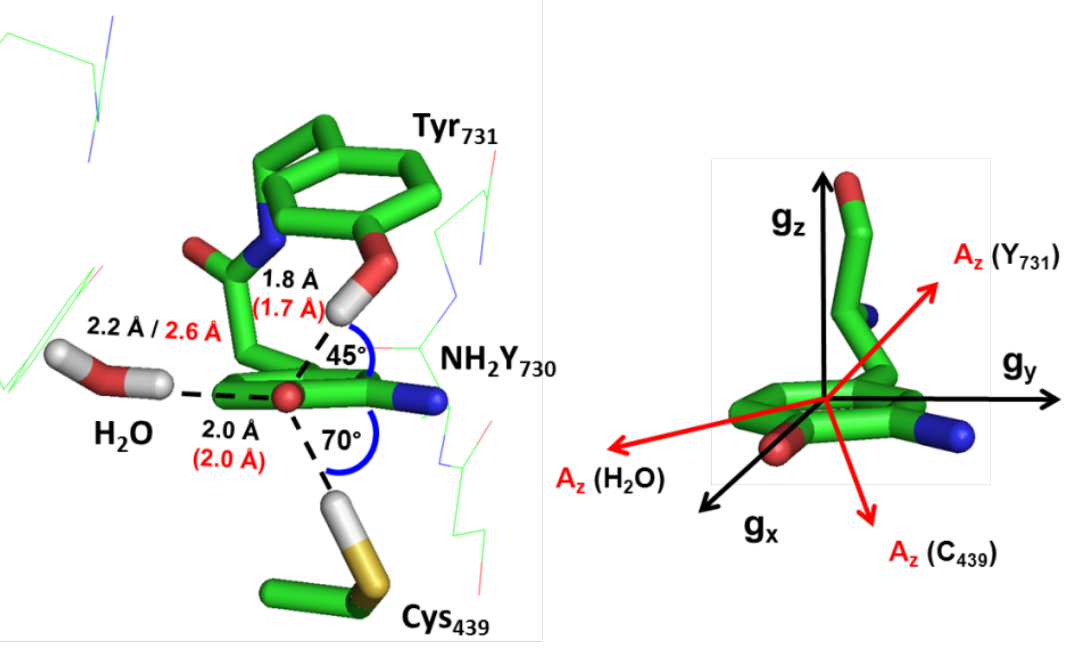

Our study revealed in case of both spin traps two hydrogen bonds which are directed towards the PCET pathway residues, $\alpha-Y_{731}$ and $\alpha-C_{439}$ as well as $\beta-Y_{356}$ and $\alpha-Y_{730}$. Furthermore deuterons from surrounding $\mathrm{D}_{2} \mathrm{O}$ molecules could be detected which may play a role in controlling the PCET kinetics between the pathway residues. The recently published $\mathrm{X}$-ray structures of $\alpha 2-\mathrm{NH}_{2} \mathrm{Y}_{730}$ and $\alpha 2-\mathrm{NH}_{2} \mathrm{Y}_{731}$ set the boundaries for the assignment of the water molecules. High-field ENDOR revealed for $\mathrm{NH}_{2} \mathrm{Y}_{730}$ one water molecule which is placed in a $0^{\circ}-D$ distance of $2.6 \AA$ parallel to the ring plane. The water molecule resides on the opposite side of the $\mathrm{NH}_{2}$-group in agreement with the $\mathrm{X}$-ray crystallographic data. DFT cluster calculations based on the resting state of $\alpha 2-\mathrm{NH}_{2} \mathrm{Y}_{730}$ could confirm two hydrogen- 
bonds pointing towards $\alpha-Y_{731}$ and $\alpha-C_{439}$ in a distance of 1.8 and $2.0 \AA$. The difference to the distances obtained from high-field ENDOR simulations were maximally $0.1 \AA$. However the experimentally obtained distance to the deuteron of the water molecule was longer when compared with the DFT study. A $0.4 \AA$ discrepancy between active and resting state suggests that the water molecule may have a role as a trigger for an $\mathrm{H}$-atom transfer event between $\mathrm{NH}_{2} \mathrm{Y}_{730}\left(\mathrm{Y}_{730}\right)$ and $\mathrm{C}_{439}$ or $\mathrm{NH}_{2} \mathrm{Y}_{730}\left(\mathrm{Y}_{730}\right)$ and $\mathrm{Y}_{731}$. DFT calculations where systematically the number of atoms was increased (in form of amino acids and water molecules) resulted in the same g-values as obtained from $180 \mathrm{GHz}$ EPR experiments $\left(g_{x}=2.0052, g_{y}=2.0042\right.$ and $\left.g_{z}=2.0022\right)$ supporting further an external hydrogen network around $\mathrm{NH}_{2} \mathrm{Y}_{730^{\circ}}$. Most surprisingly nearly the same g-values are obtained for $\alpha-\mathrm{NH}_{2} \mathrm{Y}_{731}{ }^{\bullet}$ and for $\beta-\mathrm{NH}_{2} \mathrm{Y}_{356}{ }^{\bullet}$ suggesting a similar hydrogen network around both spin probes. Orientation selective highfield ENDOR results on $\alpha-\mathrm{NH}_{2} \mathrm{Y}_{731}{ }^{\bullet}$ are hereby in agreement with a hydrogen-bond network similar to $\alpha-\mathrm{NH}_{2} \mathrm{Y}_{730} \cdot$ with an additional water molecule placed near the $\alpha / \beta$ interface.

The investigation of the hydrogen-bond network around $\mathrm{NH}_{2} \mathrm{Y}_{730}$ and $\mathrm{NH}_{2} \mathrm{Y}_{731}{ }^{\bullet}$ in the $\alpha$-subunit and specifically the obtained orientations of hydrogen-bonds through high-field ENDOR spectroscopy indicate for both sites that an $\mathrm{H}$-atom transfer mechanism (co-linear $\mathrm{PCET}$ ) is present during activity of E.coli ribonucleotide reductase. 
$\underline{\text { References }}$ 


\section{References}

\section{Chapter 1}

1. Jordan A \& Reichard P (1998) Ribonucleotide reductases. Annu Rev Biochem 67: 7198.

2. $\quad$ Stubbe J \& van Der Donk WA (1998) Protein Radicals in Enzyme Catalysis. $\underline{\text { Chem Rev }}$ 98 (2): 705-762.

3. Mao SS, et al. (1992) A model for the role of multiple cysteine residues involved in ribonucleotide reduction: amazing and still confusing. Biochemistry 31 (40): 97339743.

4. Mao SS, et al. (1992) Interaction of C225S-R1 mutant subunit of ribonucleotide reductase with $\mathrm{R} 2$ and nucleoside diphosphates: tales of a suicidal enzyme. Biochemistry 31 (40): 9744-9751.

5. Mao SS, Yu GX, Chalfoun D, \& Stubbe J (1992) Characterization of C439S-R1, a mutant of Escherichia coli ribonucleotide diphosphate reductase: evidence that C439 is a residue essential for nucleotide reduction and C439S-R1 is a protein possessing novel thioredoxin-like activity. Biochemistry 31 (40): 9752-9759.

6. Stubbe JA (1989) Protein radical involvement in biological catalysis? Annu Rev Biochem 58: 257-285.

7. $\quad$ Nordlund P \& Reichard P (2006) Ribonucleotide reductases. Annu Rev Biochem 75: 681-706.

8. Reichard P (2010) Ribonucleotide reductases: substrate specificity by allostery. Biochem Biophys Res Commun 396 (1): 19-23.

9. Atta $\mathrm{M}$, et al. (2010) S-Adenosylmethionine-dependent radical-based modification of biological macromolecules. Curr Opin Struct Biol 20 (6): 684-692.

10. Cox N, et al. (2010) A tyrosyl-dimanganese coupled spin system is the native metalloradical cofactor of the R2F subunit of the ribonucleotide reductase of Corynebacterium ammoniagenes. J Am Chem Soc 132 (32): 11197-11213.

11. Boal AK, Cotruvo JA, Jr., Stubbe J, \& Rosenzweig AC (2010) Structural basis for activation of class Ib ribonucleotide reductase. Science 329 (5998): 1526-1530.

12. Sjöberg BM (2010) Biochemistry. A never-ending story. Science 329 (5998): 14751476.

13. Jiang W, et al. (2007) A manganese(IV)/iron(III) cofactor in Chlamydia trachomatis ribonucleotide reductase. Science 316 (5828): 1188-1191.

14. Uppsten $\mathrm{M}$, et al. (2003) Structure of the large subunit of class Ib ribonucleotide reductase from Salmonella typhimurium and its complexes with allosteric effectors. J Mol Biol 330 (1): 87-97.

15. Larsson KM, et al. (2004) Structural mechanism of allosteric substrate specificity regulation in a ribonucleotide reductase. Nat Struct Mol Biol 11 (11): 1142-1149.

16. Logan DT, Andersson J, Sjöberg BM, \& Nordlund P (1999) A glycyl radical site in the crystal structure of a class III ribonucleotide reductase. Science 283 (5407): 14991504.

17. Larsson KM, Andersson J, Siöberg BM, Nordlund P, \& Logan DT (2001) Structural basis for allosteric substrate specificity regulation in anaerobic ribonucleotide reductases. Structure 9 (8): 739-750. 
18. Brown NC \& Reichard P (1969) Role of effector binding in allosteric control of ribonucleoside diphosphate reductase. J Mol Biol 46 (1): 39-55.

19. Ehrenberg A \& Reichard P (1972) Electron spin resonance of the iron-containing protein B2 from ribonucleotide reductase. J Biol Chem 247 (11): 3485-3488.

20. Licht S, Gerfen GJ, \& Stubbe J (1996) Thiyl radicals in ribonucleotide reductases. Science 271 (5248): 477-481.

21. Unkrig V, Neugebauer FA, \& Knappe J (1989) The free radical of pyruvate formatelyase. Characterization by EPR spectroscopy and involvement in catalysis as studied with the substrate-analogue hypophosphite. Eur J Biochem 184 (3): 723-728.

22. Duboc-Toia C, et al. (2003) Very high-field EPR study of glycyl radical enzymes. J Am Chem Soc 125 (1): 38-39.

23. Huyett JE, et al. (1995) Compound ES of Cytochrome-C Peroxidase Contains a Trp pCation Radical - Characterization by cw and Pulsed Q-Band Endor Spectroscopy. J Am Chem Soc 117(35): 9033-9041.

24. Bender CJ, et al. (1989) J. Am. Chem. Soc. 111: 8076.

25. Hoganson CW, Sahlin M, Sjöberg BM, \& Babcock GT (1996) Electron magnetic resonance of the tyrosyl radical in ribonucleotide reductase from Escherichia coli. $\underline{J}$ Am Chem Soc 118 (19): 4672-4679.

26. Stubbe J \& Riggs-Gelasco P (1998) Harnessing free radicals: formation and function of the tyrosyl radical in ribonucleotide reductase. Trends Biochem Sci 23 (11): 438-443.

27. Tong WH, et al. (1996) Mechanism of assembly of the diferric cluster-tyrosyl radical cofactor of Escherichia coli ribonucleotide reductase from the diferrous form of the R2 subunit. J Am Chem Soc 118 (8): 2107-2108.

28. Bollinger JM, et al. (1994) Mechanism of Assembly of the Tyrosyl Radical-Diiron(lii) Cofactor of Escherichia-Coli Ribonucleotide Reductase. 2. Kinetics of the Excess Fe ${ }^{2+}$ Reaction by Optical, Epr, and Mössbauer Spectroscopies. J Am Chem Soc 116 (18): 8015-8023.

29. Bollinger JM, et al. (1994) Mechanism of Assembly of the Tyrosyl Radical-Diiron(lii) Cofactor of Escherichia-Coli Ribonucleotide Reductase. 3. Kinetics of the Limiting Fe ${ }^{2+}$ Reaction by Optical, Epr, and Mössbauer Spectroscopies. J Am Chem Soc 116 (18): 8024-8032.

30. Fritscher J, et al. (2005) Structure of the nitrogen-centered radical formed during inactivation of $\mathrm{E}$. coli ribonucleotide reductase by 2'-azido-2'-deoxyuridine-5'diphosphate: trapping of the 3'-ketonucleotide. J Am Chem Soc 127 (21): 7729-7738.

31. Zipse $\mathrm{H}$, et al. (2009) Structure of the nucleotide radical formed during reaction of CDP/TTP with the E441Q-a2b2 of E. coli ribonucleotide reductase. J Am Chem Soc 131 (1): 200-211.

32. Lawrence CC, et al. (1999) High-field EPR detection of a disulfide radical anion in the reduction of cytidine 5'-diphosphate by the E441Q R1 mutant of Escherichia coli ribonucleotide reductase. Proc Natl Acad Sci U SA 96 (16): 8979-8984.

33. Thelander $L$ (1973) Physicochemical characterization of ribonucleoside diphosphate reductase from Escherichia coli. J Biol Chem 248 (13): 4591-4601.

34. Nordlund P, Siöberg BM, \& Eklund $H$ (1990) Three-dimensional structure of the free radical protein of ribonucleotide reductase. Nature 345 (6276): 593-598.

35. Uhlin U \& Eklund $\mathrm{H}$ (1994) Structure of ribonucleotide reductase protein R1. Nature 370 (6490): 533-539.

36. Aubert C, Vos $\mathrm{MH}$, Mathis $\mathrm{P}$, Eker AP, \& Brettel K (2000) Intraprotein radical transfer during photoactivation of DNA photolyase. Nature 405 (6786): 586-590. 
37. Kavakli IH \& Sancar A (2004) Analysis of the role of intraprotein electron transfer in photoreactivation by DNA photolyase in vivo. Biochemistry 43 (48): 15103-15110.

38. Verkhovskaya ML, Belevich N, Euro L, Wikstrom M, \& Verkhovsky MI (2008) Realtime electron transfer in respiratory complex I. Proc Natl Acad Sci U S A 105 (10): 3763-3767.

39. Igarashi RY \& Seefeldt LC (2003) Nitrogen fixation: the mechanism of the Modependent nitrogenase. Crit Rev Biochem Mol Biol 38 (4): 351-384.

40. Loll B, Kern J, Saenger W, Zouni A, \& Biesiadka J (2005) Towards complete cofactor arrangement in the 3.0 A resolution structure of photosystem II. Nature 438 (7070): 1040-1044.

41. Climent I, Sjöberg BM, \& Huang CY (1991) Carboxyl-terminal peptides as probes for Escherichia coli ribonucleotide reductase subunit interaction: kinetic analysis of inhibition studies. Biochemistry 30 (21): 5164-5171.

42. Ekberg $M$, Birgander $P, \&$ Sjöberg BM (2003) In vivo assay for low-activity mutant forms of Escherichia coli ribonucleotide reductase. J Bacteriol 185 (4): 1167-1173.

43. Ekberg $M$, Sahlin $M$, Eriksson $M$, \& Sjöberg B-M (1996) Two conserved tyrosine residues in protein $\mathrm{R} 1$ participate in an intermolecular electron transfer in ribonucleotide reductase. J. Biol. Chem. 271:20655.

44. Siegbahn PEM, Eriksson L, Himo F, \& Pavlov M (1998) Hydrogen atom transfer in ribonucleotide reductase (RNR). J. Phys. Chem. B 102: 10622-10629.

45. Ge J, Yu G, Ator MA, \& Stubbe J (2003) Pre-steady-state and steady-state kinetic analysis of E. coli class I ribonucleotide reductase. Biochemistry 42 (34): 10071-10083.

46. Bennati $M$, et al. (2003) Pulsed ELDOR spectroscopy measures the distance between the two tyrosyl radicals in the R2 subunit of the E-coli ribonucleotide reductase. $\mathrm{JAm}$ Chem Soc 125 (49): 14988-14989.

47. Schiemann O \& Prisner TF (2007) Long-range distance determinations in biomacromolecules by EPR spectroscopy. Q Rev Biophys 40 (1): 1-53.

48. Salowe S, et al. (1993) Alternative model for mechanism-based inhibition of Escherichia coli ribonucleotide reductase by 2'-azido-2'-deoxyuridine 5'-diphosphate. Biochemistry 32 (47): 12749-12760.

49. Matysik J, et al. (2000) Photochemically induced nuclear spin polarization in reaction centers of photosystem II observed by ${ }^{13} \mathrm{C}$-solid-state NMR reveals a strongly asymmetric electronic structure of the P680('+) primary donor chlorophyll. Proc Natl Acad Sci U SA 97 (18): 9865-9870.

50. Dempsey JL, Winkler JR, \& Gray HB (2010) Proton-coupled electron flow in protein redox machines. Chem Rev 110 (12): 7024-7039.

51. Bennati $M$, et al. (2005) EPR distance measurements support a model for long-range radical initiation in E. coli ribonucleotide reductase. J Am Chem Soc 127 (43): 1501415015.

52. Marcus RA \& Sutin N (1985) Electron Transfers in Chemistry and Biology. Biochim Biophys Acta 811 (3): 265-322.

53. Gray HB \& Winkler JR (2003) Electron tunneling through proteins. Q Rev Biophys 36 (3): 341-372.

54. Winkler JR, Di Bilio AJ, Farrow NA, Richards JH, \& Gray HB (1999) Electron tunneling in biological molecules. Pure App/ Chem 71 (9): 1753-1764.

55. Wang M, Gao J, Muller P, \& Giese B (2009) Electron transfer in peptides with cysteine and methionine as relay amino acids. Angew Chem Int Ed Engl 48 (23): 4232-4234. 
56. Gray HB \& Winkler JR (2005) Long-range electron transfer. Proc Natl Acad Sci U S A 102 (10): 3534-3539.

57. Cukier RI (1994) Mechanism for Proton-Coupled Electron-Transfer Reactions. J Phys Chem 98 (9): 2377-2381.

58. Turro C, Chang CK, Leroi GE, Cukier RI, \& Nocera DG (1992) Photoinduced ElectronTransfer Mediated by a Hydrogen-Bonded Interface. J Am Chem Soc 114 (10): 40134015.

59. Reece SY, Hodgkiss JM, Stubbe J, \& Nocera DG (2006) Proton-coupled electron transfer: the mechanistic underpinning for radical transport and catalysis in biology. Philos Trans R Soc Lond B Biol Sci 361 (1472): 1351-1364.

60. Costentin C, Robert M, \& Saveant JM (2007) Adiabatic and non-adiabatic concerted proton-electron transfers. Temperature effects in the oxidation of intramolecularly hydrogen-bonded phenols. J Am Chem Soc 129 (32): 9953-9963.

61. Reece SY \& Nocera DG (2009) Proton-coupled electron transfer in biology: results from synergistic studies in natural and model systems. Annu Rev Biochem 78: 673699.

62. Yee CS, Seyedsayamdost MR, Chang MC, Nocera DG, \& Stubbe J (2003) Generation of the R2 subunit of ribonucleotide reductase by intein chemistry: insertion of 3nitrotyrosine at residue 356 as a probe of the radical initiation process. Biochemistry 42 (49): 14541-14552.

63. Chang MC, Yee CS, Nocera DG, \& Stubbe J (2004) Site-specific replacement of a conserved tyrosine in ribonucleotide reductase with an aniline amino acid: a mechanistic probe for a redox-active tyrosine. J Am Chem Soc 126 (51): 1670216703.

64. Seyedsayamdost MR, Reece SY, Nocera DG, \& Stubbe J (2006) Mono-, di-, tri-, and tetra-substituted fluorotyrosines: new probes for enzymes that use tyrosyl radicals in catalysis. J Am Chem Soc 128 (5): 1569-1579.

65. Seyedsayamdost MR, Yee CS, Reece SY, Nocera DG, \& Stubbe J (2006) pH Rate profiles of FnY356-R2s ( $n=2,3,4)$ in Escherichia coli ribonucleotide reductase: evidence that $\mathrm{Y} 356$ is a redox-active amino acid along the radical propagation pathway. J Am Chem Soc 128 (5): 1562-1568.

66. Seyedsayamdost MR \& Stubbe J (2006) Site-specific replacement of Y356 with 3,4dihydroxyphenylalanine in the $\beta 2$ subunit of $E$. coli ribonucleotide reductase. $\mathrm{JAm}$ Chem Soc 128 (8): 2522-2523.

67. Seyedsayamdost MR, Xie J, Chan CT, Schultz PG, \& Stubbe J (2007) Site-specific insertion of 3-aminotyrosine into subunit $\alpha 2$ of $\mathrm{E}$. coli ribonucleotide reductase: direct evidence for involvement of $\mathrm{Y} 730$ and $\mathrm{Y731}$ in radical propagation. $\underline{\mathrm{JAm} \text { Chem }}$ Soc 129 (48): 15060-15071.

68. Seyedsayamdost MR, Chan CT, Mugnaini V, Stubbe J, \& Bennati M (2007) PELDOR spectroscopy with DOPA- $\beta 2$ and $\mathrm{NH} 2 \mathrm{Y}-\alpha 2 \mathrm{~s}$ : distance measurements between residues involved in the radical propagation pathway of $\mathrm{E}$. coli ribonucleotide reductase. J Am Chem Soc 129 (51): 15748-15749.

69. Sicoli G, Argirević T, Stubbe J, Tkach I, \& Bennati M (2010) Effects in $94 \mathrm{GHz}$ Orientation-Selected PELDOR on a Rigid Pair of Radicals with Non-Collinear Axes. Appl Magn Reson 37 (1-4): 539-548.

70. $\quad$ Xie J \& Schultz PG (2005) An expanding genetic code. Methods 36 (3): 227-238.

71. Liu CC \& Schultz PG (2010) Adding new chemistries to the genetic code. Annu Rev Biochem 79: 413-444. 
72. Alfonta L, Zhang Z, Uryu S, Loo JA, \& Schultz PG (2003) Site-specific incorporation of a redox-active amino acid into proteins. J Am Chem Soc 125 (48): 14662-14663.

73. Minnihan EC, Seyedsayamdost MR, Uhlin U, \& Stubbe J (2011) Kinetics of Radical Intermediate Formation and Deoxynucleotide Production in 3-AminotyrosineSubstituted Escherichia coli Ribonucleotide Reductases. J Am Chem Soc 133 (24): 9430-9440.

74. Neumann H, Wang K, Davis L, Garcia-Alai M, \& Chin JW (2010) Encoding multiple unnatural amino acids via evolution of a quadruplet-decoding ribosome. Nature 464 (7287): 441-444.

75. Bennati M \& Prisner TF (2005) New developments in high field electron paramagnetic resonance with applications in structural biology. Rep Prog Phys 68 (2): 411-448.

76. Prisner $T$, Rohrer M, \& MacMillan F (2001) Pulsed EPR spectroscopy: biological applications. Annu Rev Phys Chem 52:279-313.

77. Freed JH (2000) New technologies in electron spin resonance. Annu Rev Phys Chem 51:655-689.

78. Sezer D, Freed JH, \& Roux B (2009) Multifrequency electron spin resonance spectra of a spin-labeled protein calculated from molecular dynamics simulations. $\mathrm{J}$ Am Chem Soc 131 (7): 2597-2605.

79. Weber RT, Disselhorst JAJM, Prevo LJ, Schmidt J, \& Wenckebach WTH (1989) Electron spin-echo spectroscopy at $95 \mathrm{GHz}$. Journal of Magnetic Resonance (1969) 81 (1): 129144.

80. Brezgunov A, et al. (1991) Pulsed EPR in 2-mm band. Appl Magn Reson 2 (4): 715728.

81. Prisner TF, Un S, \& Griffin RG (1992) Pulsed Esr at 140 Ghz. Israel J Chem 32 (2-3): 357-363.

82. Hertel MM, Denysenkov VP, Bennati $M$, \& Prisner TF (2005) Pulsed 180-GHz EPR/ENDOR/PELDOR spectroscopy. Magn Reson Chem 43 Spec no.:S248-255.

83. Smirnova Tl, et al. (1997) High-frequency (95 GHz) EPR spectroscopy to characterize spin adducts. J Phys Chem B 101 (19): 3877-3885.

84. Stowell $\mathrm{MH}$, et al. (1997) Light-induced structural changes in photosynthetic reaction center: implications for mechanism of electron-proton transfer. Science 276 (5313): 812-816.

85. Persson AL, Sahlin M, \& Sjoberg BM (1998) Cysteinyl and substrate radical formation in active site mutant E441Q of Escherichia coli class I ribonucleotide reductase. J Biol Chem 273 (47): 31016-31020.

86. Hyde JS \& Froncisz W (1982) The role of microwave frequency in EPR spectroscopy of copper complexes. Annu Rev Biophys Bioeng 11:391-417.

87. Lyubenova S, et al. (2009) Multifrequency Pulsed Electron Paramagnetic Resonance on Metalloproteins. Accounts of Chemical Research 43 (2): 181-189.

88. Nesmelov YE \& Thomas DD (2010) Protein structural dynamics revealed by sitedirected spin labeling and multifrequency EPR. Biophys Rev 2 (2): 91-99.

89. Feher G (1956) Observation of Nuclear Magnetic Resonances Via the Electron Spin Resonance Line. Phys Rev 103 (3): 834-835.

90. Hyde JS, Chen JCW, \& Freed JH (1968) Electron-Electron Double Resonance of Free Radicals in Solution. J Chem Phys 48 (9): 4211-4219.

91. Mims WB (1965) Pulsed Endor Experiments. Proc R Soc Lon Ser-A 283 (1395): $452-$ 457.

92. Davies ER (1974) A new pulse endor technique. Physics Letters A 47 (1): 1-2. 
93. Bar G, et al. (2001) High-frequency $(140-\mathrm{GHz})$ time domain EPR and ENDOR spectroscopy: the tyrosyl radical-diiron cofactor in ribonucleotide reductase from yeast. J Am Chem Soc 123 (15): 3569-3576.

94. Gerfen GJ, et al. (1993) High-frequency (139.5 GHz) EPR spectroscopy of the tyrosyl radical in Escherichia coli ribonucleotide reductase. J Am Chem Soc 115 (14): 64206421.

95. Liu A, Barra A-L, Rubin H, Lu G, \& Gräslund A (2000) Heterogeneity of the Local Electrostatic Environment of the Tyrosyl Radical in Mycobacterium tuberculosis Ribonucleotide Reductase Observed by High-Field Electron Paramagnetic Resonance. J Am Chem Soc 122 (9): 1974-1978.

96. Allard P, et al. (1996) Characterization of a New Tyrosyl Free Radical in Salmonella typhimurium Ribonucleotide Reductase with EPR at 9.45 and $245 \mathrm{GHz}$. $\underline{\mathrm{J} \text { Am Chem }}$ Soc 118 (4): 895-896.

97. Schmidt PP, Andersson KK, Barra AL, Thelander L, \& Graslund A (1996) High field EPR studies of mouse ribonucleotide reductase indicate hydrogen bonding of the tyrosyl radical. J Biol Chem 271 (39): 23615-23618.

98. van Dam PJ, et al. (1998) High-Frequency EPR and Pulsed Q-Band ENDOR Studies on the Origin of the Hydrogen Bond in Tyrosyl Radicals of Ribonucleotide Reductase R2 Proteins from Mouse and Herpes Simplex Virus Type 1. J Am Chem Soc 120 (20): 5080-5085.

\section{Chapter 2}

1. Ekberg $M$, Sahlin $M$, Eriksson $M$, \& Sjöberg BM (1996) Two conserved tyrosine residues in protein $\mathrm{R} 1$ participate in an intermolecular electron transfer in ribonucleotide reductase. J Biol Chem 271 (34): 20655-20659.

2. Chang MC, Yee CS, Stubbe J, \& Nocera DG (2004) Turning on ribonucleotide reductase by light-initiated amino acid radical generation. Proc Natl Acad Sci U SA 101 (18): 6882-6887.

3. Reece SY, Seyedsayamdost MR, Stubbe J, \& Nocera DG (2007) Photoactive peptides for light-initiated tyrosyl radical generation and transport into ribonucleotide reductase. J Am Chem Soc 129 (27): 8500-8509.

4. Liu CC \& Schultz PG (2010) Adding new chemistries to the genetic code. Annu Rev Biochem 79:413-444.

5. $\quad$ Xie J \& Schultz PG (2005) An expanding genetic code. Methods 36(3): 227-238.

6. Wang L \& Schultz PG (2004) Expanding the genetic code. Angew Chem Int Ed Engl 44 (1): 34-66.

7. Wang L, Brock A, Herberich B, \& Schultz PG (2001) Expanding the genetic code of Escherichia coli. Science 292 (5516): 498-500.

8. Minnihan EC, Seyedsayamdost MR, Uhlin U, \& Stubbe J (2011) Kinetics of Radical Intermediate Formation and Deoxynucleotide Production in 3-AminotyrosineSubstituted Escherichia coli Ribonucleotide Reductases. J Am Chem Soc 133 (24): 9430-9440.

9. Jovanovic SV, Steenken S, Tosic M, Marjanovic B, \& Simic MG (1994) Flavonoids as Antioxidants. J Am Chem Soc 116 (11): 4846-4851. 
10. DeFelippis MR, et al. (1991) Electrochemical properties of tyrosine phenoxy and tryptophan indolyl radicals in peptides and amino acid analogs. The Journal of Physical Chemistry 95 (8): 3416-3419.

11. Wang $L$, Zhang Z, Brock A, \& Schultz PG (2003) Addition of the keto functional group to the genetic code of Escherichia coli. Proc Natl Acad Sci U S A 100 (1): 56-61.

12. Zhang Z, et al. (2003) A new strategy for the site-specific modification of proteins in vivo. Biochemistry 42 (22): 6735-6746.

13. Jackson JC, Duffy SP, Hess KR, \& Mehl RA (2006) Improving nature's enzyme active site with genetically encoded unnatural amino acids. J Am Chem Soc 128 (34): 1112411127.

14. Ryu Y \& Schultz PG (2006) Efficient incorporation of unnatural amino acids into proteins in Escherichia coli. Nat Methods 3(4):263-265.

15. Minnihan EC, Seyedsayamdost MR, \& Stubbe J (2009) Use of 3-aminotyrosine to examine the pathway dependence of radical propagation in Escherichia coli ribonucleotide reductase. Biochemistry 48 (51): 12125-12132.

16. Seyedsayamdost MR, Xie J, Chan CT, Schultz PG, \& Stubbe J (2007) Site-specific insertion of 3-aminotyrosine into subunit $\alpha 2$ of $\mathrm{E}$. coli ribonucleotide reductase: direct evidence for involvement of $\mathrm{Y} 730$ and $\mathrm{Y} 731$ in radical propagation. $\underline{\mathrm{J} \text { Am Chem }}$ Soc 129 (48): 15060-15071.

\section{Chapter 3}

1. Seyedsayamdost MR, Xie J, Chan CT, Schultz PG, \& Stubbe J (2007) Site-specific insertion of 3-aminotyrosine into subunit alpha2 of $\mathrm{E}$. coli ribonucleotide reductase: direct evidence for involvement of $\mathrm{Y} 730$ and $\mathrm{Y} 731$ in radical propagation. $\underline{J \text { Am Chem }}$ Soc 129 (48): 15060-15071.

2. Seyedsayamdost MR, Chan CT, Mugnaini V, Stubbe J, \& Bennati M (2007) PELDOR spectroscopy with DOPA- $\beta 2$ and $\mathrm{NH}_{2} \mathrm{Y}-\alpha 2 \mathrm{~s}$ : distance measurements between residues involved in the radical propagation pathway of E. coli ribonucleotide reductase. J Am Chem Soc 129 (51): 15748-15749.

3. Minnihan EC, Seyedsayamdost MR, Uhlin U, \& Stubbe J (2011) Kinetics of Radical Intermediate Formation and Deoxynucleotide Production in 3-AminotyrosineSubstituted Escherichia coli Ribonucleotide Reductases. J Am Chem Soc 133 (24): 9430-9440.

4. $\quad$ Neta P \& Fessenden RW (1974) J. Phys. Chem. 78:523.

5. Dixon WT, Hoyle PM, \& Murphy D (1978) J. Chem. Soc. Faraday II 74:2027.

6. Dixon WT, M. M, \& Murphy D (1974) J. Chem. Soc. Faraday II 70:1713.

7. Simandi LI, Barna TM, Korecz L, \& Rockenbauer A (1993) Tetrahedron Lett. 34:717.

8. $\quad$ Sur SK \& Colpa JP (1989) Organometallics 8:2749.

9. Loth K \& GrafF (1981) Helvetica. Chim. Acta 64:1910.

10. Pesavento RP \& van der Donk WA (2001) Tyrosyl radical cofactors. Adv Protein Chem 58:317-385.

11. Lendzian F (2005) Structure and interactions of amino acid radicals in class I ribonucleotide reductase studied by ENDOR and high-field EPR spectroscopy. Biochim Biophys Acta 1707(1):67-90.

12. Loth K \& Graf F (1981) Structure and Dynamics of Intramolecular Hydrogen-Bonds in Radicals - Substituent, Steric and Solvent Effects. Helv Chim Acta 64 (6): 1910-1929. 
13. Alabugin IV \& Manoharan M (2007) Rehybridization as a general mechanism for maximizing chemical and supramolecular bonding and a driving force for chemical reactions. J Comput Chem 28(1):373-390.

14. Yokoyama K, Uhlin U, \& Stubbe J (2010) Site-Specific Incorporation of 3-Nitrotyrosine as a Probe of pKa Perturbation of Redox-Active Tyrosines in Ribonucleotide Reductase. J Am Chem Soc 132(24):8385-8397.

15. Fairman JW, et al. (2011) Structural basis for allosteric regulation of human ribonucleotide reductase by nucleotide-induced oligomerization. Nat Struct Mol Biol 18 (3): 316-322.

16. $\underline{X u ~ H, ~ F a b e r ~ C, ~ U c h i k i ~ T, ~ R a c c a ~ J, ~ \& ~ D e a l w i s ~ C ~(2006) ~ S t r u c t u r e s ~ o f ~ e u k a r y o t i c ~}$ ribonucleotide reductase I define gemcitabine diphosphate binding and subunit assembly. Proc Natl Acad Sci U S A 103 (11): 4028-4033.

17. Huyett JE, et al. (1995) J. Am. Chem. Soc. 117:9033.

18. Bleifuss G, et al. (2001) Biochemistry 40:15362-15368.

19. Hulsebosch RJ, et al. (1997) J. Am. Chem. Soc. 119:8685-8694.

20. Bender CJ, et al. (1989) J. Am. Chem. Soc. 111:8076.

21. Hoganson CW, Sahlin M, Sjöberg B-M, \& Babcock GT (1996) J. Am. Chem. Soc. 118: $4672-4679$.

22. Un S (2005) The g-values and hyperfine coupling of amino acid radicals in proteins: comparison of experimental measurements with ab initio calculations. Magn Reson Chem 43 Spec no.:S229-236.

23. Himo F, Eriksson LA, Blomberg MRA, \& Siegbahn PEM (2000) Int. J. Quantum Chem. 76: 714-723.

24. Gerfen GJ, et al. (1993) High-frequency (139.5 GHz) EPR spectroscopy of the tyrosyl radical in Escherichia coli ribonucleotide reductase. J Am Chem Soc 115 (14): 64206421.

25. Brezgunov AY, et al. (1992) Magnetic-Resonance Parameters and Structure of Substituted Stannyloxyphenoxyl Radicals - Investigation by Means of Esr in the 2-Mm Band. J Struct Chem 33 (5): 678-685.

26. Gerfen GJ, et al. (1996) High-frequency electron paramagnetic resonance spectroscopy of the apogalactose oxidase radical. J Phys Chem 100 (41): 1673916748.

27. Lee Y-K, Whittaker MM, \& Whittaker JW (2008) The Electronic Structure of the CysTyr• Free Radical in Galactose Oxidase Determined by EPR Spectroscopy ${ }^{\dagger}$. Biochemistry 47 (25): 6637-6649.

28. Un S, Atta M, Fontecave M, \& Rutherford AW (1995) g-Values as a Probe of the Local Protein Environment: High-Field EPR of Tyrosyl Radicals in Ribonucleotide Reductase and Photosystem II. J Am Chem Soc 117 (43): 10713-10719.

29. Seyedsayamdost MR \& Stubbe J (2006) Site-specific replacement of Y356 with 3,4dihydroxyphenylalanine in the $\beta 2$ subunit of $E$. coli ribonucleotide reductase. $\underline{J \mathrm{Am}}$ Chem Soc 128 (8): 2522-2523. 


\section{Chapter 4}

1. Eriksson M, et al. (1997) Binding of allosteric effectors to ribonucleotide reductase protein R1: reduction of active-site cysteines promotes substrate binding. Structure 5 (8): 1077-1092.

2. Seyedsayamdost MR, Chan CT, Mugnaini V, Stubbe J, \& Bennati M (2007) PELDOR spectroscopy with DOPA-beta2 and NH2Y-alpha2s: distance measurements between residues involved in the radical propagation pathway of $\mathrm{E}$. coli ribonucleotide reductase. J Am Chem Soc 129 (51): 15748-15749.

3. Minnihan EC, Seyedsayamdost MR, Uhlin U, \& Stubbe J (2011) Kinetics of Radical Intermediate Formation and Deoxynucleotide Production in 3-AminotyrosineSubstituted Escherichia coli Ribonucleotide Reductases. J Am Chem Soc 133 (24): 9430-9440.

4. Ge J, Yu G, Ator MA, \& Stubbe J (2003) Pre-steady-state and steady-state kinetic analysis of E. coli class I ribonucleotide reductase. Biochemistry 42 (34): 10071-10083.

5. Ekberg $M$, Sahlin $M$, Eriksson $M$, \& Sjöberg BM (1996) Two conserved tyrosine residues in protein $\mathrm{R} 1$ participate in an intermolecular electron transfer in ribonucleotide reductase. J Biol Chem 271 (34): 20655-20659.

6. Chang MC, Yee CS, Stubbe J, \& Nocera DG (2004) Turning on ribonucleotide reductase by light-initiated amino acid radical generation. Proc Natl Acad Sci U SA 101 (18): 6882-6887.

7. Reece SY, Seyedsayamdost MR, Stubbe J, \& Nocera DG (2007) Photoactive peptides for light-initiated tyrosyl radical generation and transport into ribonucleotide reductase. J Am Chem Soc 129 (27): 8500-8509.

8. Siegbahn PE \& Blomberg MR (2010) Quantum chemical studies of proton-coupled electron transfer in metalloenzymes. Chem Rev 110 (12): 7040-7061.

9. Farrar CT, Gerfen GJ, Griffin RG, Force DA, \& Britt RD (1997) Electronic Structure of the YD Tyrosyl Radical in Photosystem II: A High-Frequency Electron Paramagnetic Resonance Spectroscopic and Density Functional Theoretical Study. The Journal of Physical Chemistry B 101 (33): 6634-6641.

10. Himo F, Eriksson LA, Blomberg MRA, \& Siegbahn PEM (2000) Int. J. Quantum Chem. 76: 714-723.

11. Seyedsayamdost MR, Xie J, Chan CT, Schultz PG, \& Stubbe J (2007) Site-specific insertion of 3-aminotyrosine into subunit $\alpha 2$ of $\mathrm{E}$. coli ribonucleotide reductase: direct evidence for involvement of Y730 and Y731 in radical propagation. J Am Chem Soc 129 (48): 15060-15071.

12. Uhlin U \& Eklund $H$ (1994) Structure of ribonucleotide reductase protein R1. Nature 370 (6490): 533-539.

13. Fairman JW, et al. (2011) Structural basis for allosteric regulation of human ribonucleotide reductase by nucleotide-induced oligomerization. Nat Struct Mol Biol 18 (3): 316-322.

14. Wang M, Gao J, Muller P, \& Giese B (2009) Electron transfer in peptides with cysteine and methionine as relay amino acids. Angew Chem Int Ed Engl 48 (23): 4232-4234.

15. Lin J, Balabin IA, \& Beratan DN (2005) The nature of aqueous tunneling pathways between electron-transfer proteins. Science 310 (5752): 1311-1313. 
16. de la Lande A, Babcock NS, Rezac J, Sanders BC, \& Salahub DR (2010) Surface residues dynamically organize water bridges to enhance electron transfer between proteins. Proc Natl Acad Sci U S A 107 (26): 11799-11804.

\section{Materials and Methods of chapter 3 and 4}

1. Hertel MM, Denysenkov VP, Bennati M, \& Prisner TF (2005) Magn. Reson. Chem. 43:S248-S255.

2. Lawrence CC, et al. (1999) High-field EPR detection of a disulfide radical anion in the reduction of cytidine 5'-diphosphate by the E441Q R1 mutant of Escherichia coli ribonucleotide reductase. Proc. Natl. Acad. Sci. U.S.A. 96:8979-8984.

3. Stoll S \& Schweiger A (2006) EasySpin, a comprehensive software package for spectral simulation and analysis in EPR. J Magn Reson 178 (1): 42-55.

4. $\quad$ Bender CJ, et al. (1989) J. Am. Chem. Soc. 111: 8076.

5. Seyedsayamdost MR, Argirević T, Minnihan EC, Stubbe J, \& Bennati M (2009) Structural examination of the transient 3-aminotyrosyl radical on the PCET pathway of E. coli ribonucleotide reductase by multifrequency EPR spectroscopy. $\underline{\mathrm{J} \mathrm{Am} \text { Chem }}$ Soc 131 (43): 15729-15738.

6. Weigend F \& Ahlrichs R (2005) Balanced basis sets of split valence, triple zeta valence and quadruple zeta valence quality for $\mathrm{H}$ to $\mathrm{Rn}$ : Design and assessment of accuracy. Phys. Chem. Chem. Phys. 7 (18): 3297-3305.

7. Schäfer A, Huber C, \& Ahlrichs R (1994) Fully Optimized Contracted Gaussian-Basis Sets of Triple Zeta Valence Quality for Atoms Li to Kr. J. Chem. Phys. 100 (8): 58295835.

8. Perdew JP (1986) Density-functional approximation for the correlation energy of the inhomogeneous electron gas. Phys. Rev. B 33 (12): 8822-8824.

9. Perdew JP (1986) Erratum: Density-functional approximation for the correlation energy of the inhomogeneous electron gas. Phys. Rev. B 34 (10): 7406.

10. Becke $A D$ (1988) Density-functional exchange-energy approximation with correct asymptotic behavior. Phys. Rev. A 38 (6): 3098-3100.

11. Lee C, Yang W, \& Parr RG (1988) Development of the Colle-Salvetti correlation-energy formula into a functional of the electron density. Phys. Rev. B 37(2):785-789.

12. Becke AD (1993) Density-functional thermochemistry. III. The role of exact exchange. J. Chem. Phys. 98 (1): 5648-5652.

13. Barone V (EPR-II. Recent Advances in Density Functional Methods, Part I.

14. Kutzelnigg W, Fleischer U, \& Schindler M (1990) NMR Basic Principles and Progress, Vol. 23. (Springer, Berlin).

15. Weigend F (2002) A fully direct RI-HF algorithm: Implementation, optimised auxiliary basis sets, demonstration of accuracy and efficiency. Physical Chemistry Chemical Physics 4 (18): 4285-4291.

16. Grimme $S$ (2006) Semiempirical GGA-type density functional constructed with a longrange dispersion correction. J. Comput. Chem. 27 (15): 1787-1799.

17. Baker J (1986) An algorithm for the location of transition states. J. Comput. Chem. 7 (4): 385-395.

18. Neese F (2007) ORCA, an ab initio, density functional and semiempirical program package. University of Bonn, Germany. 
Appendix 


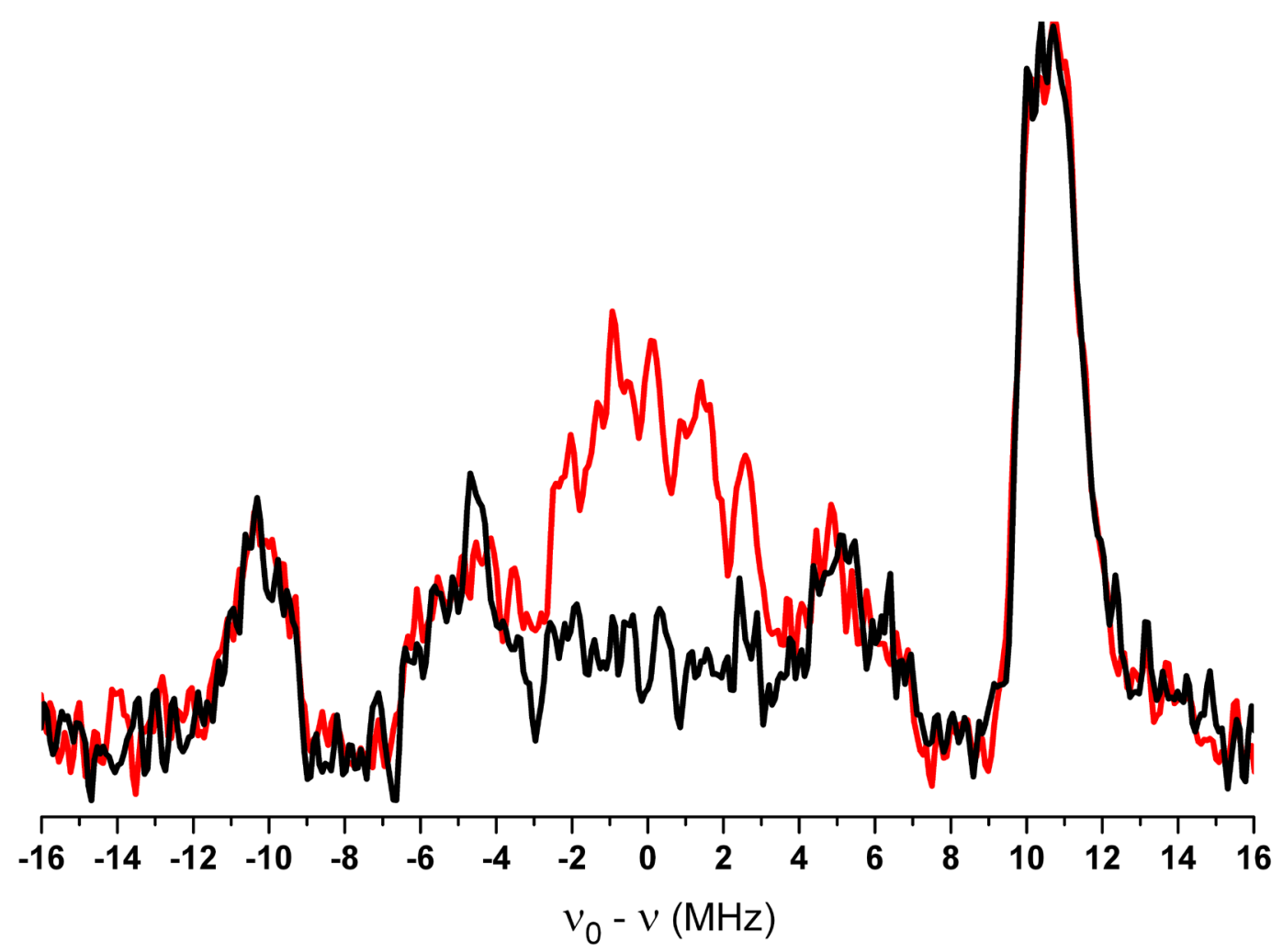

Figure A-1: ${ }^{1} \mathrm{H}$ Davies ENDOR spectra recorded at $34 \mathrm{GHz}$ on $\mathrm{ND}_{2} \mathrm{Y}_{731}{ }^{\bullet}$ with different preparation pulses (30 seconds quenching time). Experimental conditions (for red spectrum): $\mathrm{T}=70 \mathrm{~K}, \mathrm{MW}(\pi / 2)=$ $24 \mathrm{~ns}$, prep $(\pi)=128 \mathrm{~ns}, \tau=240 \mathrm{~ns}$, RF pulse $=15 \mu \mathrm{s}$, repetition time $=5 \mathrm{~ms}, 6650$ scans, $24 \mathrm{~h}$ acquisition time. Experimental conditions (for black spectrum): $\mathrm{T}=70 \mathrm{~K}, \mathrm{MW}(\pi / 2)=20 \mathrm{~ns}$, prep $(\pi)=$ $64 \mathrm{~ns}, \tau=240 \mathrm{~ns}, \mathrm{RF}$ pulse $=15 \mu \mathrm{s}$, repetition time $=5 \mathrm{~ms}, 6600$ scans, $24 \mathrm{~h}$ acquisition time. 


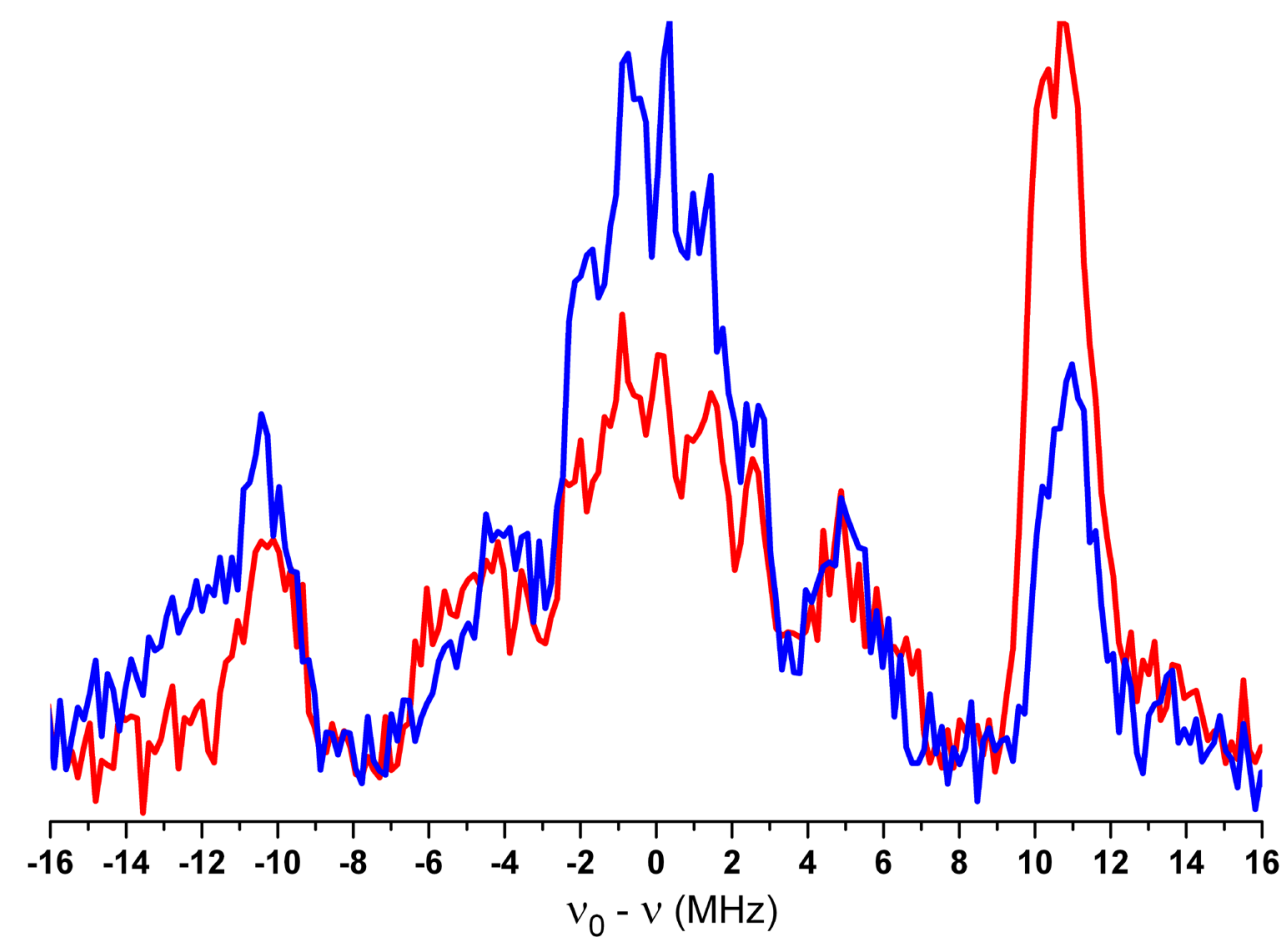

Figure A-2: ${ }^{1} \mathrm{H}$ Davies ENDOR spectra recorded at $34 \mathrm{GHz}$ on $\mathrm{ND}_{2} \mathrm{Y}_{731}{ }^{\circ}$ with different $\mathrm{RF}$ amplifiers (30 seconds quenching time). Experimental conditions (for red spectrum): $\mathrm{T}=70 \mathrm{~K}, \mathrm{MW}(\pi / 2)=24 \mathrm{~ns}$, prep $(\pi)=128 \mathrm{~ns}, \tau=240 \mathrm{~ns}$, RF pulse $=15 \mu \mathrm{s}$, repetition time $=5 \mathrm{~ms}, 6650$ scans, $24 \mathrm{~h}$ acquisition time, amplifier = Barthel. Experimental conditions (for blue spectrum): $\mathrm{T}=70 \mathrm{~K}, \mathrm{MW}(\pi / 2)=28 \mathrm{~ns}$, $\operatorname{prep}(\pi)=128 \mathrm{~ns}, \quad \tau=300 \mathrm{~ns}, \mathrm{RF}$ pulse $=15 \mu \mathrm{s}$, repetition time $=5 \mathrm{~ms}, 15149$ scans, $48 \mathrm{~h}$ acquisition time, amplifier $=$ AR (amplifier research). 


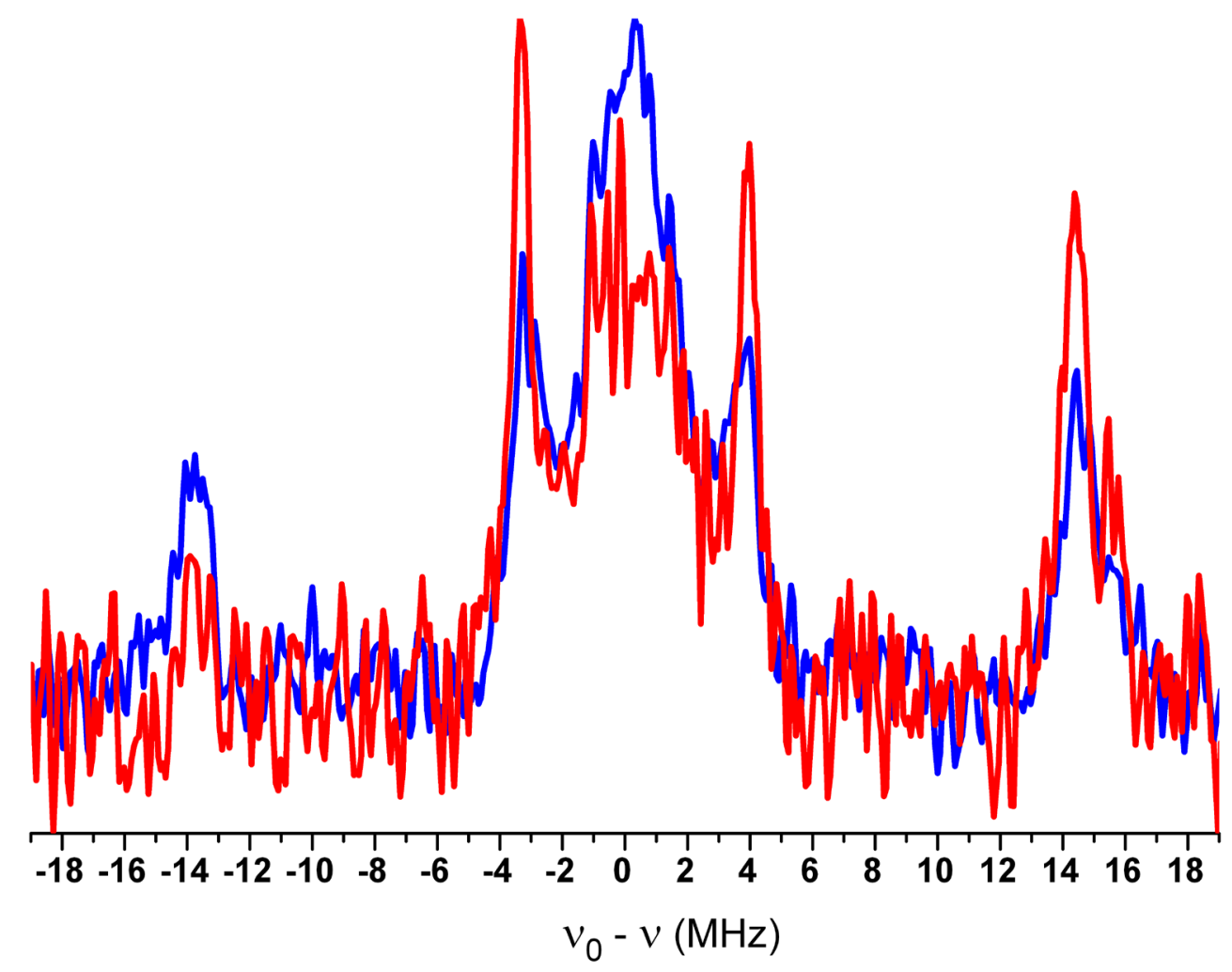

Figure A-3: ${ }^{1} \mathrm{H}$ Davies ENDOR spectra recorded at $34 \mathrm{GHz}$ on $\mathrm{ND}_{2} \mathrm{Y}_{730}{ }^{\circ}$ with different $\mathrm{RF}$ amplifiers (40 seconds quenching time). Experimental conditions (for red spectrum): $\mathrm{T}=70 \mathrm{~K}, \mathrm{MW}(\pi / 2)=20 \mathrm{~ns}$, prep $(\pi)=128 \mathrm{~ns}, \tau=240 \mathrm{~ns}$, RF pulse $=15 \mu \mathrm{s}$, repetition time $=5 \mathrm{~ms}, 15500$ scans, $48 \mathrm{~h}$ acquisition time, amplifier $=$ Barthel. Experimental conditions (for blue spectrum): $\mathrm{T}=70 \mathrm{~K}, \mathrm{MW}(\pi / 2)=20 \mathrm{~ns}$, prep $(\pi)=128 \mathrm{~ns}, \tau=300 \mathrm{~ns}$, RF pulse $=15 \mu \mathrm{s}$, repetition time $=5 \mathrm{~ms}, 15100$ scans, $48 \mathrm{~h}$ acquisition time, amplifier $=$ AR (amplifier research). 


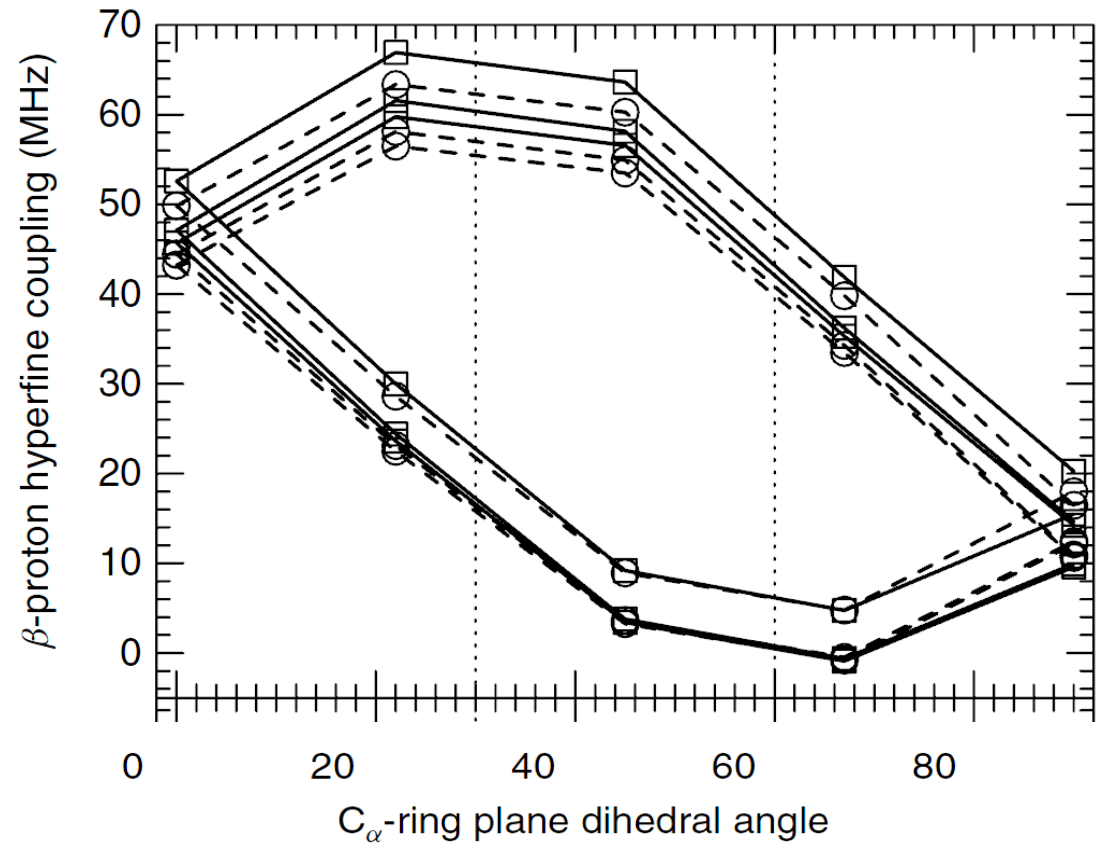

Figure A-4: The dependence of the two $\mathrm{H}_{\beta}$-methylene proton hyperfine couplings of $p$-ethylphenoxy radical on the $\mathrm{C}_{\alpha}$-ring plane dihedral angle. The dashed lines and circles are values for the isolated radical and the solid lines and squares are for the $p$-ethylphenoxy radical-imidazole complex. The structures were geometry-optimized while constraining the dihedral angle. All results are from B3LYP/EPR-III calculations. 


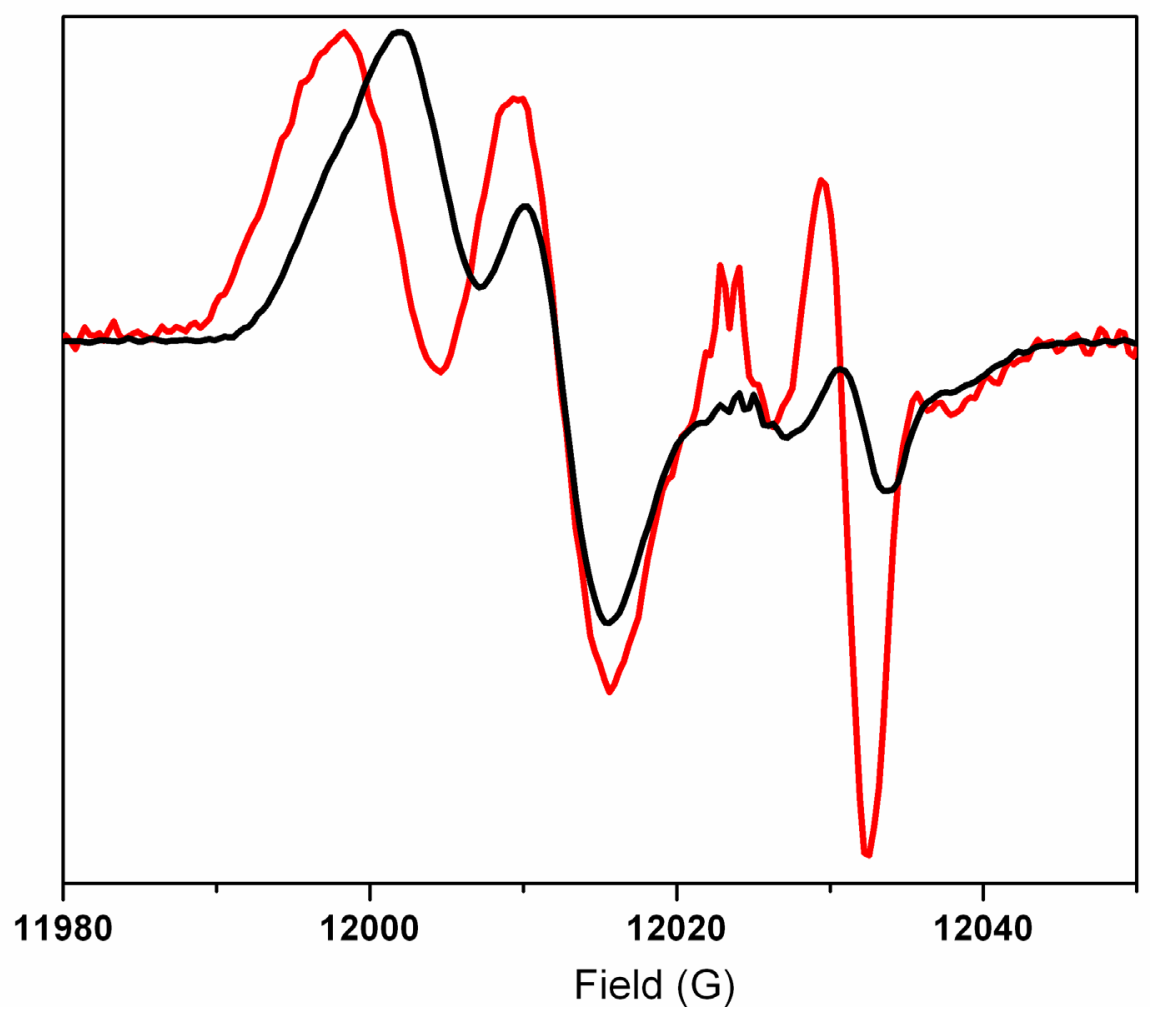

Figure A-5: Comparison of $34 \mathrm{GHz}$ pulsed derivative EPR spectra of $\mathrm{NH}_{2} \mathrm{Y}_{730^{\circ}}$ (red trace) and $\mathrm{NH}_{2} \mathrm{Y}_{731}$ • (black trace) in $\mathrm{D}_{2} \mathrm{O}$ assay buffer, respectively. The additional peak at $\sim 12030 \mathrm{G}$ is the e-centre of the Q-band quartz tube (see also Figure 4-6 in Chapter 4).

Experimental conditions (red spectrum): $\mathrm{T}=70 \mathrm{~K}, \mathrm{MW}(\pi / 2)=32 \mathrm{~ns}, \tau=300 \mathrm{~ns}, \mathrm{MW}(\pi)=64 \mathrm{~ns}$, repetition time $=5 \mathrm{~ms}$, shots $/$ point $=50$, scans $=635$. Experimental conditions (black spectrum): $\mathrm{T}=70 \mathrm{~K}, \mathrm{MW}(\pi / 2)=24 \mathrm{~ns}, \tau=240 \mathrm{~ns}, \mathrm{MW}(\pi)=48 \mathrm{~ns}$, repetition time $=5 \mathrm{~ms}$, shots $/$ point $=50$, scans $=1200$. 


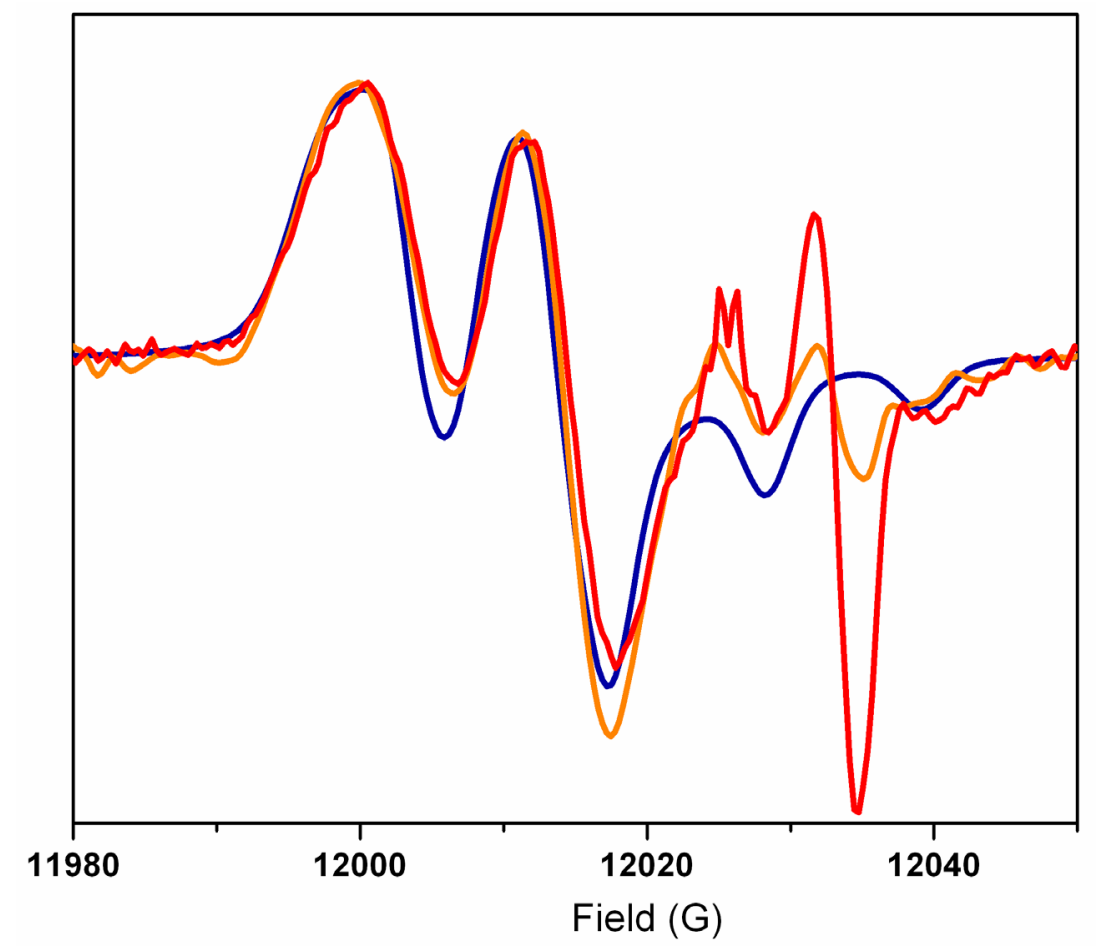

Figure A-6: Simulation (blue trace with SimFonia) of $34 \mathrm{GHz}$ pulsed derivative EPR spectra of $\mathrm{NH}_{2} \mathrm{Y}_{730^{\circ}}$ at $70 \mathrm{~K}$ (red trace) and at $10 \mathrm{~K}$ after $\mathrm{Y}_{122}{ }^{\circ}$ subtraction (orange trace) in $\mathrm{D}_{2} \mathrm{O}$ assay buffer. The additional peak at $~ 12030 \mathrm{G}$ is the e-centre of the Q-band quartz tube (see also Figure 4-6 in Chapter 4).

Experimental conditions (red spectrum): $\mathrm{T}=70 \mathrm{~K}, \mathrm{MW}(\pi / 2)=32 \mathrm{~ns}, \tau=300 \mathrm{~ns}, \mathrm{MW}(\pi)=64 \mathrm{~ns}$, repetition time $=5 \mathrm{~ms}$, shots $/$ point $=50$, scans $=635$.

Experimental conditions (orange spectrum): $\mathrm{T}=10 \mathrm{~K}, \mathrm{MW}(\pi / 2)=20 \mathrm{~ns}, \tau=200 \mathrm{~ns}, \mathrm{MW}(\pi)=40 \mathrm{~ns}$, repetition time $=150 \mathrm{~ms}$, shots $/$ point $=5$, scans $=100$. 


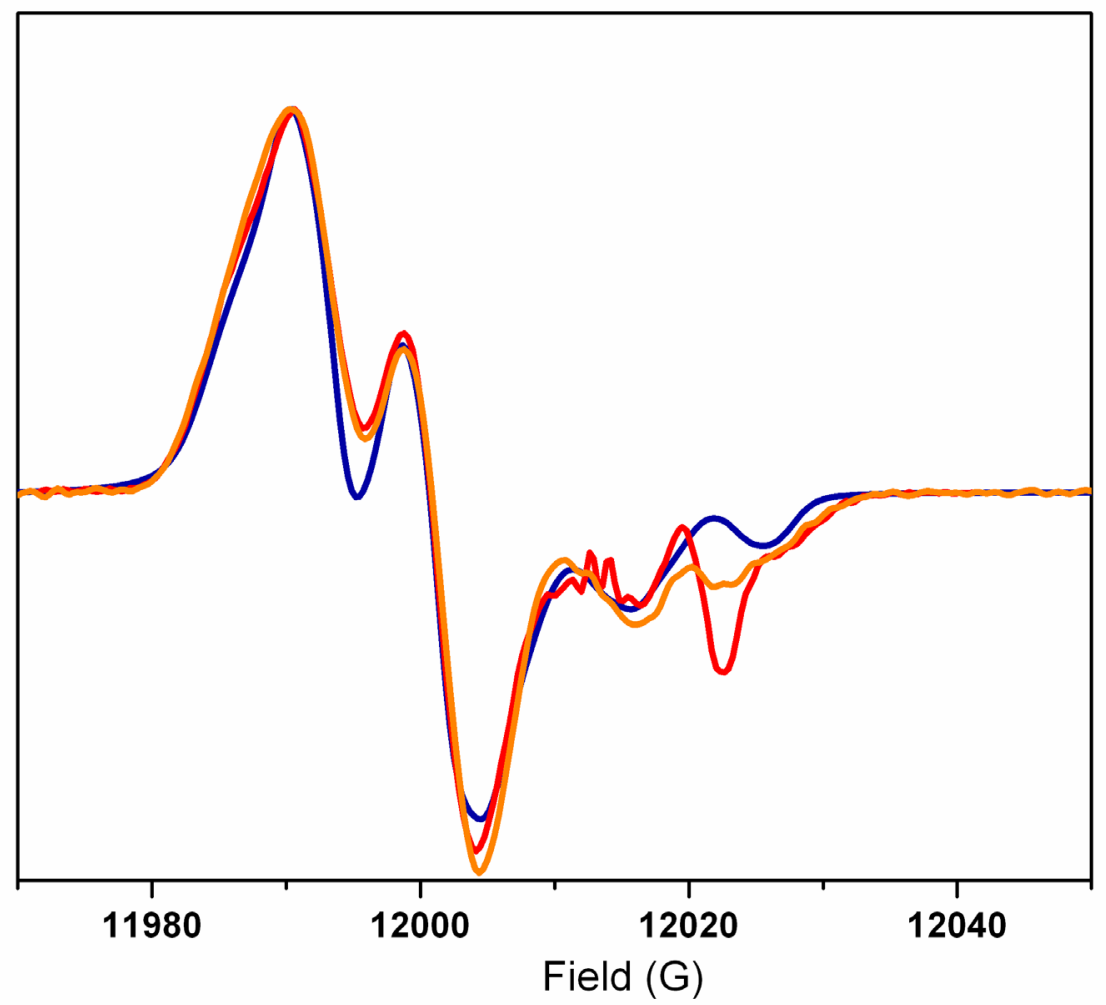

Figure A-7: Simulation (blue trace with SimFonia) of $34 \mathrm{GHz}$ pulsed derivative EPR spectra of $\mathrm{NH}_{2} \mathrm{Y}_{731}{ }^{\bullet}$ at $70 \mathrm{~K}$ (red trace) and at $10 \mathrm{~K}$ after $\mathrm{Y}_{122}{ }^{\circ}$ subtraction (orange trace) in $\mathrm{D}_{2} \mathrm{O}$ assay buffer. The additional peak at $\sim 12030 \mathrm{G}$ is the e-centre of the Q-band quartz tube (see also Figure 4-6 in Chapter 4).

Experimental conditions (red spectrum): $\mathrm{T}=70 \mathrm{~K}, \mathrm{MW}(\pi / 2)=24 \mathrm{~ns}, \tau=240 \mathrm{~ns}, \mathrm{MW}(\pi)=48 \mathrm{~ns}$, repetition time $=5 \mathrm{~ms}$, shots $/$ point $=50$, scans $=1200$.

Experimental conditions (orange spectrum): $\mathrm{T}=10 \mathrm{~K}, \mathrm{MW}(\pi / 2)=20 \mathrm{~ns}, \tau=200 \mathrm{~ns}, \mathrm{MW}(\pi)=40 \mathrm{~ns}$, repetition time $=150 \mathrm{~ms}$, shots $/$ point $=5$, scans $=200$. 

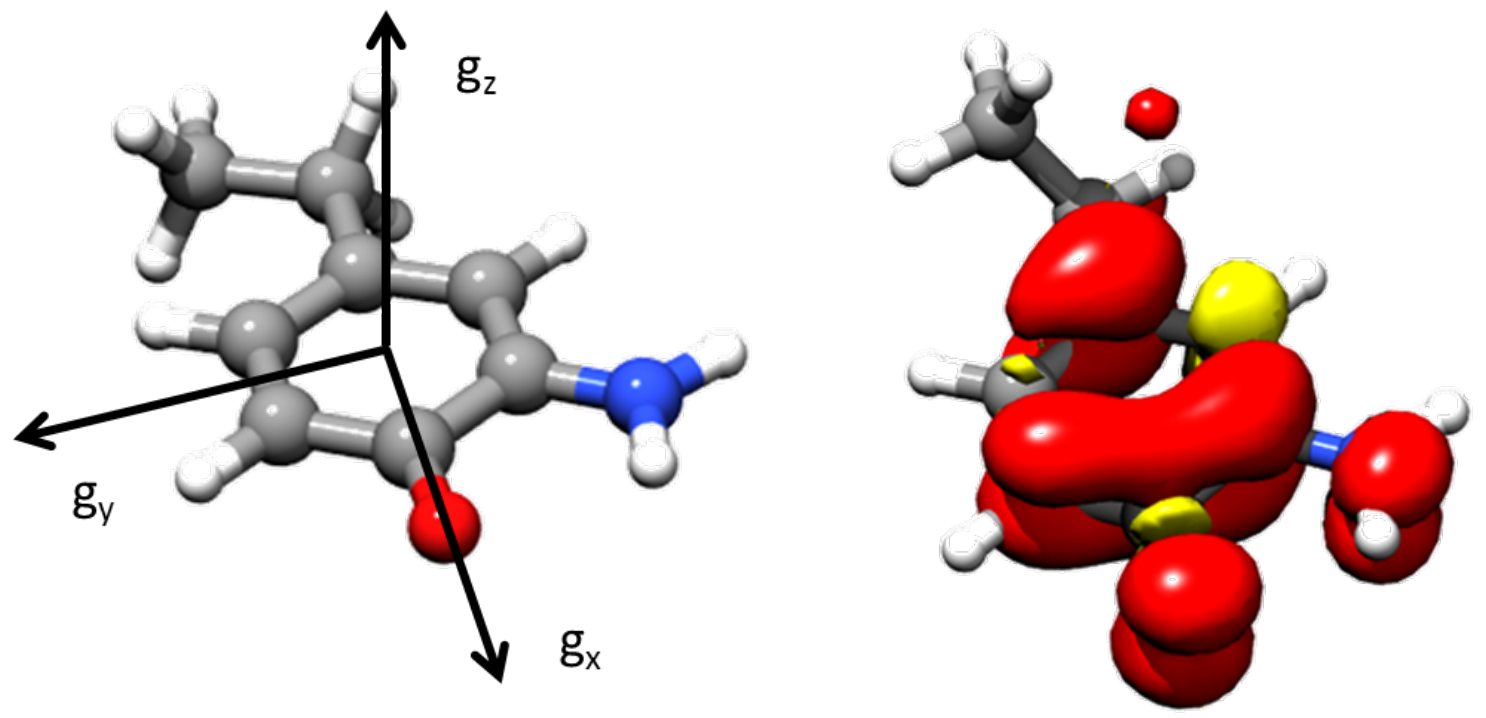

Figure A-8: Optimized structure of the 4-ethyl-2-amino-tyrosyl radical with the indicated g-Tensor (left). Spin density distribution of the 4-ethyl-2-amino-tyrosyl radical calculated with the B3LYP/EPR III level.

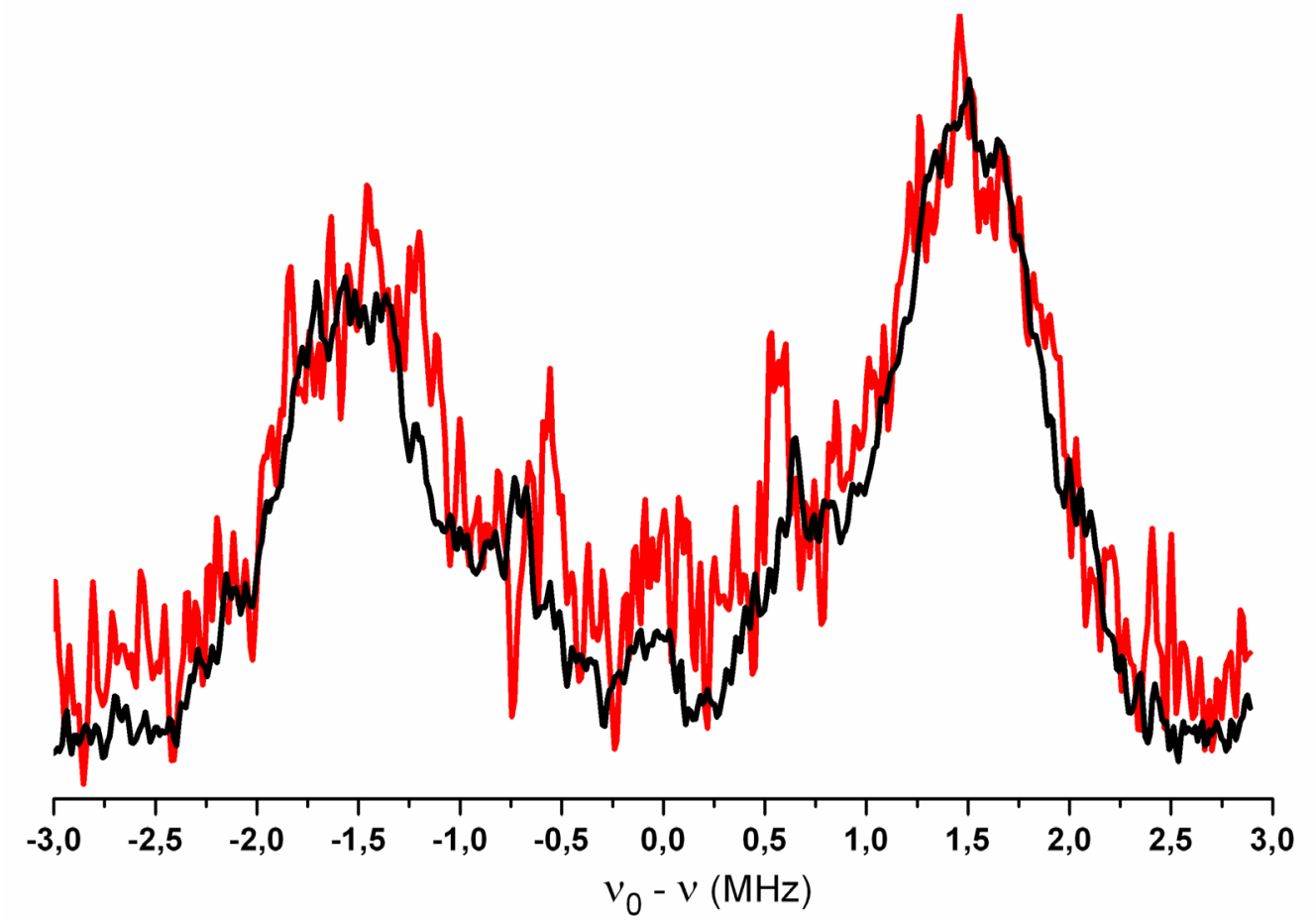

Figure A-9: Comparison of $\mathrm{ND}_{2} \mathrm{Y}_{730^{\circ}}$ and $\mathrm{ND}_{2} \mathrm{Y}_{731}{ }^{\circ}$ ENDOR spectra measured at $34 \mathrm{GHz}$ with a $\tau$-value of $100 \mathrm{~ns}$. Experimental conditions (for ENDOR on $\mathrm{ND}_{2} Y_{730^{\circ}}$ (red spectrum)): $\mathrm{T}=7.5 \mathrm{~K}, \mathrm{MW}$ pulse $(\pi / 2)$ $=20 \mathrm{~ns}, \tau=100 \mathrm{~ns}, \mathrm{RF}$ pulse $=40 \mu \mathrm{s}$, repetition time $=150 \mathrm{~ms}, 2335$ scans, $40 \mathrm{~h}$ acquisition time. Experimental conditions (for ENDOR on $N D_{2} Y_{731^{\circ}}$ (black spectrum)): $\quad \mathrm{T}=7.5 \mathrm{~K}$, MW pulse $(\pi / 2)=20$ $\mathrm{ns}, \tau=100 \mathrm{~ns}$, RF pulse $=40 \mu \mathrm{s}$, repetition time $=150 \mathrm{~ms}, 1012$ scans, $20 \mathrm{~h}$ acquisition time. 
Curriculum vitae 


\section{Curriculum vitae}

First and last name:

Address:

Date of birth:

Nationality:

Family status:

Languages:
Tomislav Argirević

Annastrasse 66

37075 Göttingen

Germany

28.04.1978

German

single

german, englisch, serbian, french (basics)

Scientific education:

$10 / 2001-12 / 2006$

$04 / 2007-10 / 2007$

Since 11/2007
Studies of Chemistry:

University of Frankfurt am Main

Diploma thesis: „Characterization of the conformational ensemble of unfolded lysozyme through NMR spectroscopy"

Research stay:

MIT (Massachusetts Institute of Technology)

Department of chemistry

Doctoral work:

University of Göttingen (GAUSS program)

Carried out at the Max Planck Institute for Biophysical Chemistry in Göttingen in the research group of Prof. Bennati

Doctoral thesis: „High-field EPR and ENDOR spectroscopy for proton-coupled electron transfer investigations in E.coli Ribonucleotide Reductase" 
Fellowships:

$02 / 2007-02 / 2010$

$02 / 2010-10 / 2011$

2009

2010

2011
International Graduate School fellowship 1422

Max Planck Society fellowship

\section{Publications:}

M. R. Seyedsayamdost, T. Argirević, E. C. Minnihan, J. Stubbe and M. Bennati

J. Am. Chem. Soc. 2009, 131, 15729 -15738.

G. Sicoli, T. Argirević, J. Stubbe, I. Tkach and M. Bennati Appl. Magn. Reson. 2010, 37, 539-548.

T. Argirević, C. Riplinger, J. Stubbe, F. Neese and M. Bennati

To be submitted 